Available online at www.sciencedirect.com

science (W)irect*

\title{
Nanocrystalline materials and coatings
}

\author{
S.C. Tjong*, Haydn Chen \\ Department of Physics and Materials Science, City University of Hong Kong, Tat Chee Avenue, Kowloon, Hong Kong
}

Available online 10 August 2004

\begin{abstract}
In recent years, near-nano (submicron) and nanostructured materials have attracted increasingly more attention from the materials community. Nanocrystalline materials are characterized by a microstructural length or grain size of up to about $100 \mathrm{~nm}$. Materials having grain size of $\sim 0.1$ to $0.3 \mu \mathrm{m}$ are classified as submicron materials. Nanocrystalline materials exhibit various shapes or forms, and possess unique chemical, physical or mechanical properties. When the grain size is below a critical value $(\sim 10-20 \mathrm{~nm})$, more than $50 \mathrm{vol} . \%$ of atoms is associated with grain boundaries or interfacial boundaries. In this respect, dislocation pile-ups cannot form, and the Hall-Petch relationship for conventional coarse-grained materials is no longer valid. Therefore, grain boundaries play a major role in the deformation of nanocrystalline materials. Nanocrystalline materials exhibit creep and super plasticity at lower temperatures than conventional micro-grained counterparts. Similarly, plastic deformation of nanocrystalline coatings is considered to be associated with grain boundary sliding assisted by grain boundary diffusion or rotation. In this review paper, current developments in fabrication, microstructure, physical and mechanical properties of nanocrystalline materials and coatings will be addressed. Particular attention is paid to the properties of transition metal nitride nanocrystalline films formed by ion beam assisted deposition process.

(C) 2004 Elsevier B.V. All rights reserved.
\end{abstract}

Keywords: Nanostructure; Synthesis; Mechanical properties; Grain size; Processing-structure-property; Nanocrystalline coatings; Ion beam

\section{Introduction}

Nanomaterials are experiencing a rapid development in recent years due to their existing and/or potential applications in a wide variety of technological areas such as electronics, catalysis, ceramics, magnetic data storage, structural components etc. To meet the technological demands in these areas, the size of the materials should be reduced to the nanometer scale. For example, the miniaturization of functional electronic devices demands the placement or assembly of nanometer scale components into well-defined structures. As the size reduces into the nanometer range, the materials exhibit peculiar and interesting mechanical and physical properties, e.g. increased mechanical strength, enhanced diffusivity, higher specific heat and electrical resistivity compared to conventional coarse grained counterparts [1]. Nanomaterials can be classified into nanocrystalline materials and nanoparticles. The former are polycrystalline bulk materials with grain sizes in the nanometer range (less than $100 \mathrm{~nm}$ ), while the latter refers to ultrafine dispersive particles with diameters below $100 \mathrm{~nm}$. Nanoparticles are generally considered as the building blocks of bulk nanocrystalline materials. Research in nanomaterials is a multidisciplinary effort that involves interaction between researchers in the field of physics, chemistry, mechanics and materials science, or even biology and medicine. It has been stimulated by the

\footnotetext{
* Corresponding author. Tel.: +852 2788 7831; fax: +852 27887830.

E-mail address: aptjong@cityu.edu.hk (S.C. Tjong).
} 
interest for basic scientific investigations and their technological applications. Nanomaterials and most of the applications derived from them are still in an early stage of technical development. There are several issues that remain to be addressed before nanomaterials will become potentially useful for industrial sectors. These issues include synthesis of high purity materials with large yield economically and environmentally, characterization of new structures and properties of nanophase materials, fabrication of dense products from nanoparticles with full density and less contamination, and retention of the ultrafine grain size in service in order to preserve the mechanical properties associated with the nanometer scale.

Novel fabrication technology of nanoparticles is versatile and includes a wide range of vapor, liquid and solid state processing routes. Available techniques for the synthesis of nanoparticles via vapor routes range from physical vapor deposition and chemical vapor deposition to aerosol spraying. The liquid route involves sol-gel and wet chemical methods. The solid state route preparation takes place via mechanical milling and mechanochemical synthesis. Each method has its own advantages and shortcomings. Among these, mechanical milling and spray conversion processing are commonly used to produce large quantities of nanopowders.

Nanoparticles synthesized from several routes may have different internal structures that would affect the properties of materials consolidated from them. Processing nanoparticles into fully dense, bulk products or coatings which retain the nanometer scale grain size is rather difficult to achieve in practice. Due to their high specific surface areas, nanoparticles exhibit a high reactivity and strong tendency towards agglomeration. Moreover, rapid grain growth is likely to occur during processing at high temperatures. As unique properties of nanocrystaline materials derived from their fine grain size, it is of crucial importance to retain the microstructure at a nanometer scale during consolidation to form bulk materials. It is also noticed that pores are generated in bulk nanocrystalline materials consolidated from nanoparticles prepared by inert-gas condensation. Such nanopores can lead to a decrease in Young's modulus of consolidated nanocrystalline materials [2]. Electrodeposited samples are believed to be free from porosity, but they contain certain impurities and texture that may degrade their mechanical performances. Therefore, controlling these properties during synthesis and subsequent consolidation procedures are the largest challenges facing researchers.

The unique properties of nanocrystalline materials are derived from their large number of grain boundaries compared to coarse-grained polycrystalline counterpartes. In nanocrystalline solids, a large fraction of atoms (up to 49\%) are boundary atoms. Thus the interface structure plays an important role in determining the physical and mechanical properties of nanocrystalline materials. Huang et al. [3] reported that nanocrystalline copper has a much higher resistivity and a larger temperature dependence of the resistivity than bulk copper. They attributed this effect to the grainboundary enhanced scattering of electrons. Nanocrystalline metals have been found to exhibit creep and superplasticity with high strain rates at lower temperatures than their micro-grained counterparts. High strain-rate superplasticity at lower temperatures is of practical interest because it can offer an efficiently near-net-shape forming technique to industrial sectors. Despite recent advances in the development of nanocrystalline materials, much work remains to be done to achieve a basic understanding of their deformation and fracture behavior. Nanocrystalline metals generally exhibit significantly higher yield strength and reduced tensile elongation relative to their microcrystalline counterparts. The hardness and yield strength tend to increase with decreasing grain size down to a critical value (ca. $20 \mathrm{~nm}$ ). When the grain size is below $20 \mathrm{~nm}$, strength appears to decrease with further grain refinement. At this stage, dislocation sources inside the grains can hardly exists. This implies that dislocation pile-ups cannot form and the Hall-Petch relationship for conventional coarsergrained materials is no longer valid. Instead, inverse Hall-Petch effect, i.e. softening is obtained when 
the grain size is reduced. The softening behavior of nanocrystalline materials is a subject of considerable debate. Several mechanisms have been proposed to explain the anomalous deformation behavior of nanocrystalline materials with the grain size below the critical value. These include grainboundary sliding, grain-boundary diffusion, the triple junction effect, presence of nanopores and impurities, etc. Therefore, comprehensive understanding of the processing-structure-property relationships is essential in the development of novel nanomaterials with unique properties for structural engineering applications. As dense nanocrystalline materials with grain size smaller than $20 \mathrm{~nm}$ are difficult to acquire, molecular dynamics (MD) modeling has been used to simulate the interfacial structure and mechanical deformation mechanism of such materials. Computer simulations play a critical role in advancing our understanding of atomic level and deformation structures that are not accessible by experimental routes.

Nanocrystalline coatings with grain sizes in the nanometer range are also known to exhibit superior hardness and strength. The search for nanostructured coatings is driven by the improvement in coating technologies and the availability of various kinds of synthesized nanopowders. Such nanopowders can be used as feedstock materials for thermal spray processes; these include plasma spraying and high-velocity oxygen fuel (HVOF) spraying. Thermal spraying involves particle melting, rapid cooling and consolidation in a single-step operation. Thermal-sprayed nanocrystalline coatings with moderate hardness are found to possess better wear performances than their counterparts fabricated from microcrystalline powders. HVOF is particularly suited to deposit dense nanocrystalline ceramic coatings as opposed to plasma spraying because of its lower spraying temperature. Today, HVOF allows tailoring nanocrystalline coatings with low porosity, higher bond strength and increased wear properties.

In the past decade, favorable applications have been found for hard and wear-resistant ceramic coatings in industrial sectors. Transition metal nitride coatings are of particular interest due to their high hardness, thermal stability, attractive appearance and chemical inertness. Conventional nitride coatings have been prepared by physical and chemical vapor deposition. Nanocrystalline coatings of transition metal nitrides can be deposited on substrate materials by means of ion beam assisted deposition. The process is based on simultaneous ion bombardment of the growing physical vapor deposited film using an independent ion source. This technique permits the deposition of nanocrystalline nitride films at lower temperatures with better coating-substrate adhesion.

The improvement of tool materials coated with transition metal nitrides has led to interest in developing superhard coatings for wear protection under complex loads and aggressive environments. Optimal microstructural design and materials selection permit chemical, physical and mechanical characteristics can be tailored for specific applications. Superhard coatings having hardness values above $40 \mathrm{GPa}$ are obtainable in multilayer structures with the period of the superlattice (bilayer thickness) within the nanometer regime. The enhancement of superhardness is attributed to a difference in shear modulus between two layer materials and to the presence of sharp interfaces between the layers. In another approach, nanocomposite coatings with superhardness $\geq 40 \mathrm{GPa}$ can be prepared by dispersing the transition metal nitride nanoparticles in an amorphous covalent nitride matrix $(\leq 1 \mathrm{~nm})$. In terms of MD computer simulations, grain-boundary accommodation mechanisms such as grain-boundary sliding and diffusion are considered to be the main factors causing the softening of nanocrystalline materials with grain sizes below $10 \mathrm{~nm}$. Blocking of grain-boundary sliding of nanocrystalline grains embedded in a thin amorphous matrix is believed to be responsible for superhardness of the nanocomposite coatings.

Because of the multidsiplinary nature of nanomaterials, it would be difficult to cover all areas of interest. In this review article, we address and focus the discussions on the following subjects, namely nanoparticles, nanocrystalline materials and coatings. 


\section{Nanostructure}

One of the most critical characteristics of nanoparticles is their very high surface-to-volume ratio, i.e. large fractions of surface atoms. Thus, large fractions of surface atoms together with ultra-fine size and shape effects make nanoparticles exhibit distinctly different properties from the bulk. The evolution of nanoparticles from a vapor or liquid phase involves three fundamental steps: nucleation, coalescence and growth. When the concentration of building blocks (atoms or ions) of a solid becomes sufficiently high, they aggregate into small clusters through homogeneous nucleation. With continuous supply of the building blocks, these clusters tend to coalesce and grow to form a larger cluster assembly. Thus, the nanoparticles are often built-up from a full-shell cluster of atoms having cubic or hexagonal closed-packed structure. Such a structure can be constructed from a central atom surrounded by a first shell of 12, a second of 42, a third of 92 atoms, etc. The number of atoms in nth shell is $10 n^{2}+2$ (Table 1). The coordination number of surface atoms is 9 or smaller, and different from their bulk counterparts, i.e. 12. With decreasing size of the nanoparticles, the percentage

Table 1

The relation between the total number of atoms in full shell clusters and the percentage of surface atoms (reprinted from [5] with permission from John Wiley \& Sons)

\begin{tabular}{|c|c|c|}
\hline Full shell clusters & Total number of atoms & Surface atoms $(\%)$ \\
\hline One shell & 13 & 92 \\
\hline Two shells & 55 & 76 \\
\hline Three shells & 147 & 63 \\
\hline Four shells & 309 & 52 \\
\hline Five shells & 561 & 45 \\
\hline Seven shells & 1415 & 35 \\
\hline
\end{tabular}




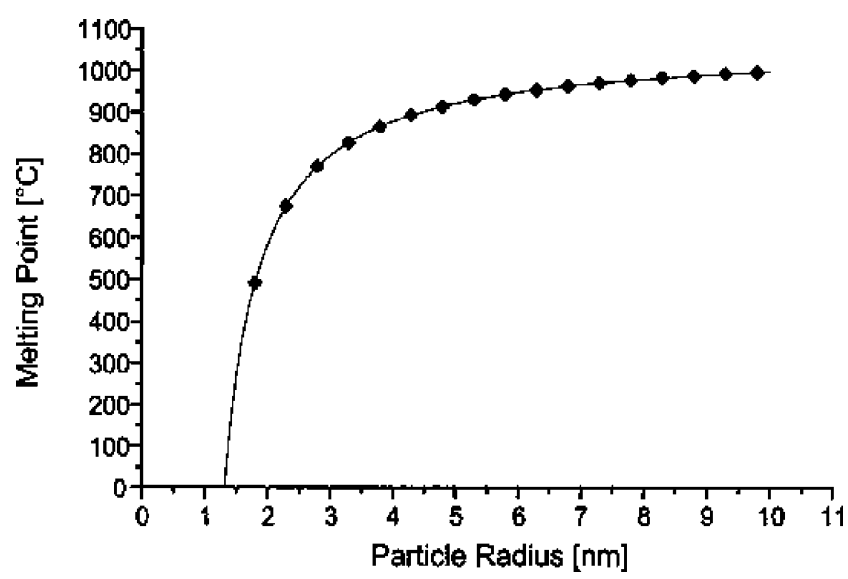

Fig. 1. Relation between the size of gold particles and their melting point (reprinted from [5] with permission from John Wiley \& Sons).

of surface atoms increases. The critical role of the size of nanoparticles in physical properties has been demonstrated experimentally in melting temperature. For example, Fig. 1 shows the typical relation between the particle size and melting point of gold particles, calculated by the method of Reifenberger and coworkers [4]. Apparently, the melting temperature of gold reduces dramatically from 1063 to $\sim 300{ }^{\circ} \mathrm{C}$ for nanoparticles with diameters of smaller than $5 \mathrm{~nm} \mathrm{[5]}$.

In general, the geometrical shape is determined by the composition and properties of the synthesized material, or by the formation mechanism of the condensed nanoparticles. For most transition metal nanoparticles, distinct structures can occur that are not characteristic of the bulk crystal structure. These structures are: cubooctahedron, icosahedron and decahedron (Fig. 2).

The first two structures are commonly observed in clusters of gold, $\mathrm{Cr}$ and other metals [6,7]. Clusters with a small number of atoms $(<150-200)$ crystallize in the form of icosahedra. The structure becomes unstable for a large number of atoms and transforms to cubooctahedra, which is just a patch of the face-centered-cubic (fcc) lattice [8,9]. A typical example is the change from icosahedral to facecentered cubic structure in gold particles [9]. The particle shapes are closely related to the crystallographic surfaces that enclose the particles. The $\left\{\begin{array}{lll}1 & 1 & 1\end{array}\right\},\left\{\begin{array}{lll}1 & 0 & 0\end{array}\right\}$ and possibly $\left\{\begin{array}{lll}1 & 1 & 0\end{array}\right\}$ surfaces of fcc metal particles, are different not only in the surface atom densities but also the electronic structure, bonding and chemical reactivities [10]. Controlling the size, shape and structure of metal nanoparticles is technologically important because of strong correlation between these parameters and optical, electrical and catalytic properties [11]. Many metals can now be processed into monodisperse nanoparticles with controlled composition and structure. They can be produced in large quantities through solution phase methods. Other morphologies with less stable facets have been achieved by adding chemical capping reagents to the synthetic systems [11-13]. For example, the shapes and sizes of $\mathrm{Pt}$ (fcc) nanoparticles such as cubic, tetrahedral and octahedral can be controlled by changes in the ratio of the concentration of the caping polymer material (sodium polyacrylate) to the concentration of the Pt ions used in the reductive synthesis of colloidal particles in solution at room temperature. The presence of the polymer in the solution of colloids is believed to have two functions. First, it stops the growth of the particles at a small size distribution. Second, it prevents individual colloidal particles from coalescing with each other [13]. The fcc nanocystals normally have $\left\{\begin{array}{lll}1 & 1 & 1\end{array}\right\}$ twins. The fc metals tend to nucleate and grow into twinned and multiply twinned particles (MTP) with their surfaces bounded by the lowest energy $\left\{\begin{array}{lll}1 & 1 & 1\end{array}\right\}$ facets [14]. Twining is the result of two subgrains sharing a common crystallographic plane. In this case, the structure of one subgrain is the mirror reflection of the 


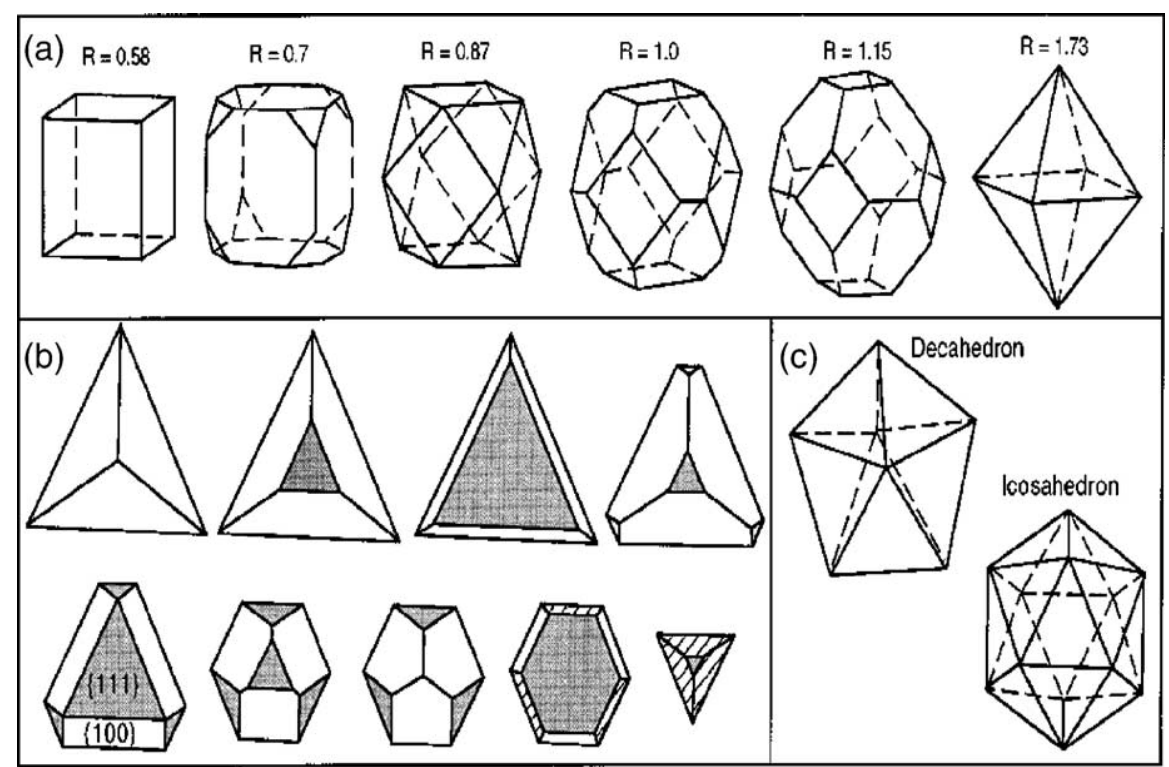

Fig. 2. (a) Geometrical shapes of cubooctahedral nanocrystals as a function of the ratio, $R$, of the growth rate along the $\left(\begin{array}{lll}1 & 0 & 0\end{array}\right)$ to that of the $\left(\begin{array}{lll}1 & 1 & 1\end{array}\right)$. (b) Evolution in shapes of a series of $\left(\begin{array}{lll}1 & 1 & 1\end{array}\right)$ based nanoparticles as the ratio $\left\{\begin{array}{lllllll}1 & 1 & 1\end{array}\right\}$ to $\left\{\begin{array}{llll}1 & 0 & 0\end{array}\right\}$ increases. The beginning particle is bounded by three $\left\{\begin{array}{lll}1 & 0 & 0\end{array}\right\}$ facets and a $\left(\begin{array}{lll}1 & 1 & 1\end{array}\right)$ base, while the final one is a $\left\{\begin{array}{lll}1 & 1 & 1\end{array}\right\}$ bounded tetrahedron. (c) Geometrical shapes of multiply twinned decahedral and icosahedral particles (reprinted from [10] with permission from American Chemical Society).

other by the twin plane. The two most typical examples of MTP are decahedron and icosahedron. As demonstrated by Wang [10], the shape of an fcc crystal is mainly determined by the ratio $(R)$ between the growth rates in the $\left(\begin{array}{lll}1 & 0 & 0\end{array}\right)$ direction to that of the $\left(\begin{array}{lll}1 & 1 & 1\end{array}\right)$. Octahedra and tetrahedral bounded by the most stable $\left\{\begin{array}{llll}1 & 1 & 1\end{array}\right\}$ planes will be formed when $R=1.73$. Perfect cubes bounded by the less stable $\left\{\begin{array}{lll}1 & 0 & 0\end{array}\right\}$ planes will result if $R$ is reduced to 0.58 . The particles with $0.87<R<1.73$ have the $\left\{\begin{array}{lll}1 & 0 & 0\end{array}\right\}$ and $\left\{\begin{array}{lll}1 & 1 & 1\end{array}\right\}$ facets, which are referred to truncated octahedron (Fig. 2). High resolution transmission electron microscopy (HRTEM) is generally known as a powerful tool to observe the morphology and structure of nanoparticles This is due to its atomic resolution capabilities to observe spatially resolved chemistry at nanoscale [15-19]. Wang et al. employed HRTEM to directly image atomic scale structures of the surfaces of platinum particles with cubic-, octahedral- and tetrahedral-like shapes prepared from a shape-controlling technique [10,19]. Fig. 3a and b shows typical HRTEM images of cubic Pt nanocrystals oriented along [ $\left[\begin{array}{lll}0 & 0 & 1\end{array}\right]$ and $\left[\begin{array}{lll}1 & 1 & 0\end{array}\right]$ directions, respectively. From Fig. 3a, the particle is bounded by $\left\{\begin{array}{lll}1 & 0 & 0\end{array}\right\}$ facets and there is no defect in the bulk of the particle. The distance between the adjacent lattice fringes is the interplanar distance of $\mathrm{Pt}(200)$, which is $0.196 \mathrm{~nm}$. The particle surface has some steps and ledges, particularly near the corner of the cube. Fig. 4a shows the HRTEM image of a truncated octahedral Pt nanoparticle oriented along [ $\left.\begin{array}{lll}1 & 1 & 0\end{array}\right]$. The HRTEM image of the octahedral-like Pt nanocrystals oriented along [ $\left[\begin{array}{lll}1 & 1 & 0\end{array}\right]$ and $\left[\begin{array}{lll}0 & 0 & 1\end{array}\right]$, respectively are shown in Fig. 4b [19]. An octahedron has eight $\left\{\begin{array}{lll}1 & 1 & 1\end{array}\right\}$ facets, four of them are edge-on when viewed along [ $\left.\begin{array}{llll}1 & 1 & 0\end{array}\right]$. If the particle is a truncated octahedron, six $\left\{\begin{array}{lll}1 & 0 & 0\end{array}\right\}$ facets are created by cutting the corners of

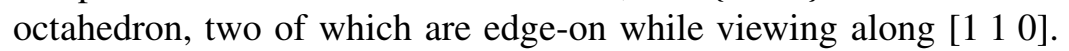

The properties of bulk nanocrystalline materials are known to be controlled by their ultra-fined grain sizes and interface boundaries. Small grain size in nanocrystalline materials produces large interfacial regions per unit volume as expected. The atomic arrangements or structures of the grain boundaries have been intensively investigated in the past decades. However, controversial results in the grain boundary structures are obtained. Some researchers suggested that the grain boundary 

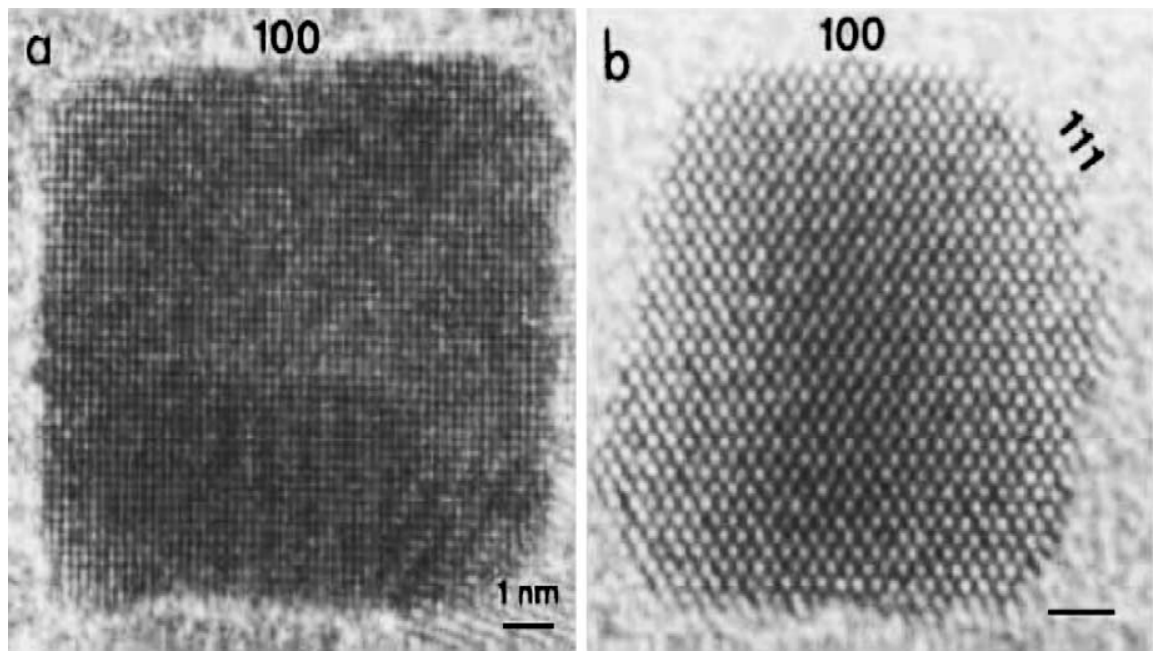

Fig. 3. HRTEM micrographs of cubic Pt nanoparticles oriented along (a) $\left[\begin{array}{lll}0 & 0 & 1\end{array}\right]$ and (b) [ $\left.\begin{array}{llll}1 & 1 & 0\end{array}\right]$, showing surface steps or ledges (reprinted from [19] with permission from Elsevier).

structure is similar to that in coarser-grained materials [20-30], while others proposed a frozen 'gaslike' structure of the grain boundaries in which the atomic arrangement at interfacial region lacks short-or long-range order [31-33]. In nanocrystalline solids a large fraction of atoms are located near grain boundaries. Assuming a grain boundary thickness of $1 \mathrm{~nm}$, materials with an average grain
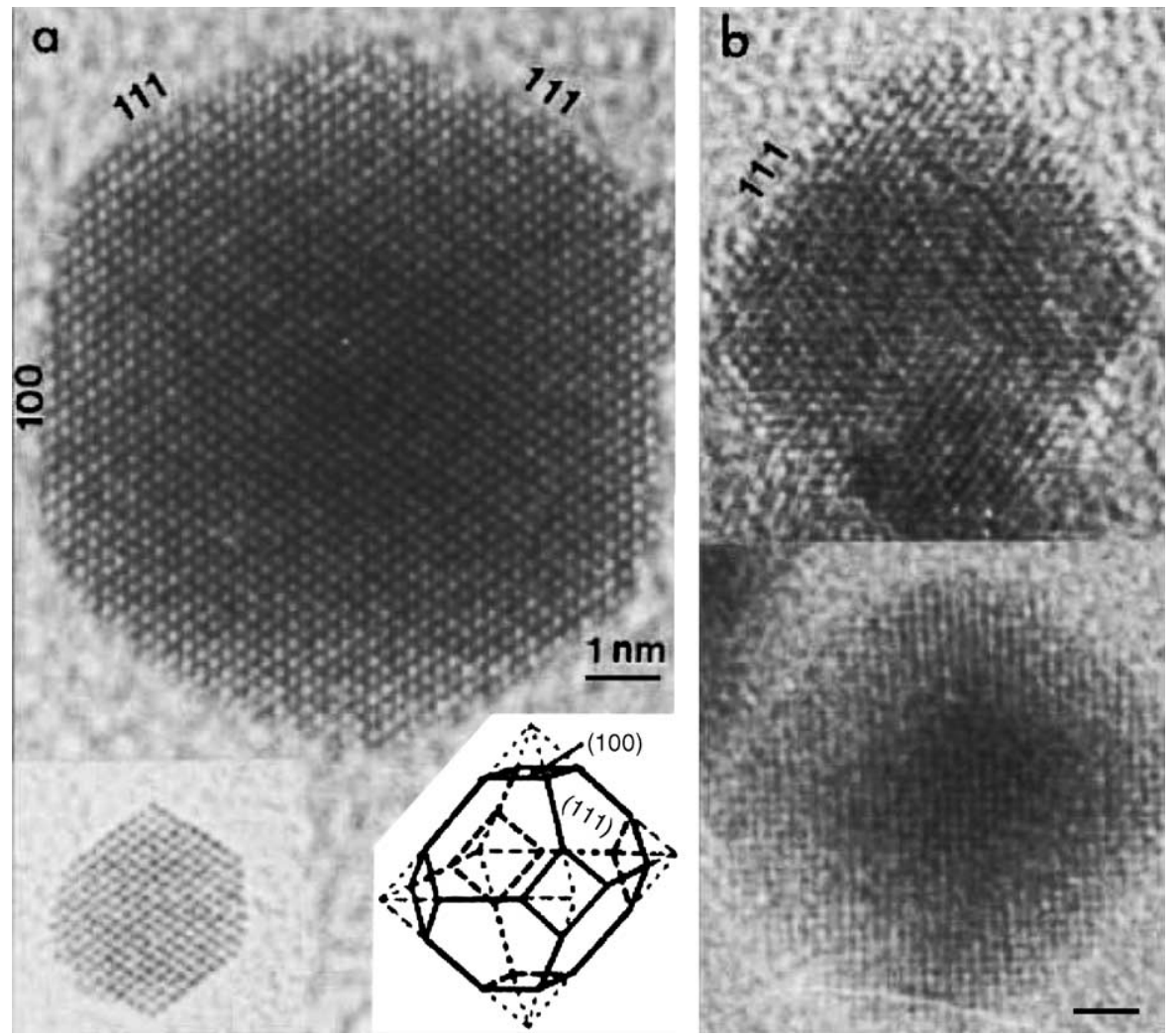

Fig. 4. HRTEM image of Pt nanoparticles with a (a) truncated octahedral shape and oriented along [1 1 0]; (b) with an

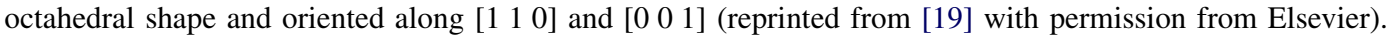




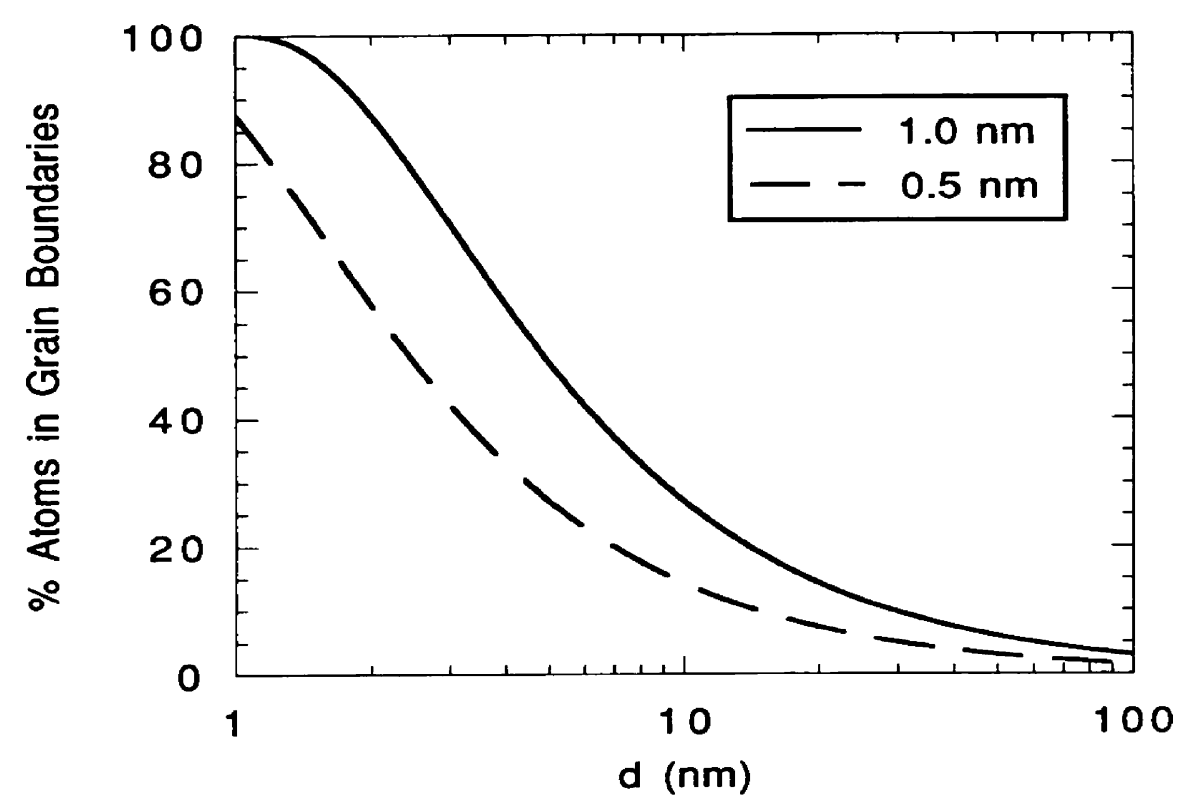

Fig. 5. Range of percentage of atoms in grain boundaries of a nanocrystalline solid as function of grain diameter, assuming that the average grain boundary thickness ranges from 0.5 to $1.0 \mathrm{~nm}$ (reprinted from [20] with permission from Annual Review Inc.)

diameter of $5 \mathrm{~nm}$ possesses $\sim 49 \%$ grain boundary atoms (Fig. 5). Earlier studies of the interfacial structures of nanocrystalline Pd with HRTEM [6] and X-ray diffraction techniques [27,28] as well as nanocrystalline $\mathrm{Cu}$ with EXAFS [27] indicated that the atomic displacements in the grain boundaries were very small, and the structures were similar to those in coarser-grained materials. Fig. 6 is the HRTEM image of the interfacial grain boundaries in nanocrystalline Pd showing the morphology of flat facets interspersed with steps [22]. On the basis of X-ray diffraction results, Gleiter and coworkers

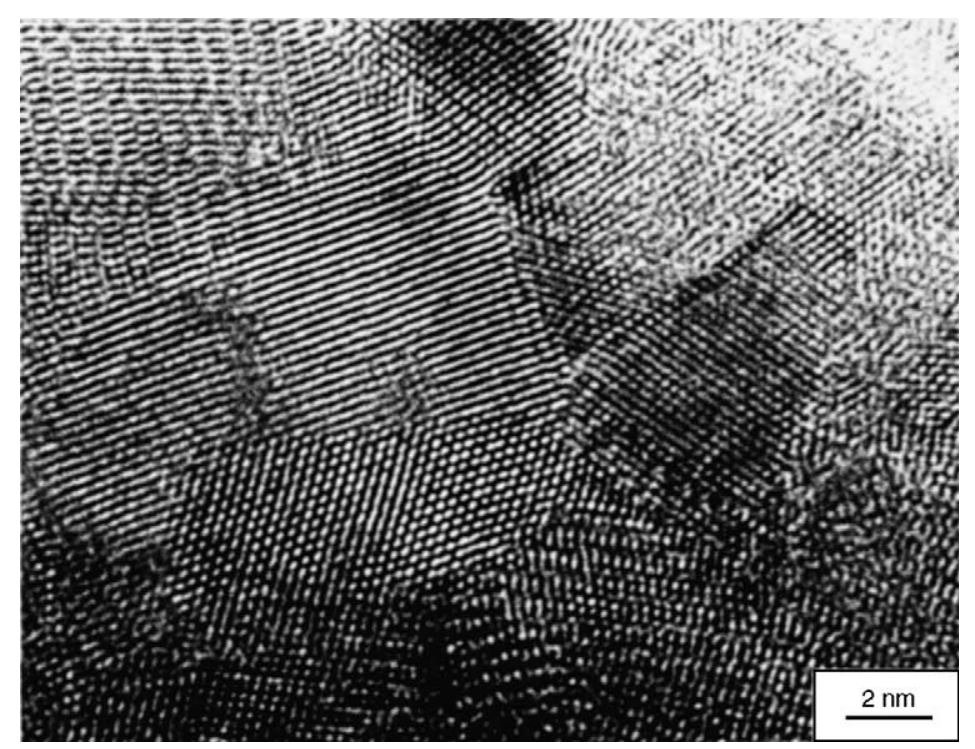

Fig. 6. HRTEM image of a region of nanocrystalline palladium containing a number of grains (reprinted from [22] with permission from Elsevier). 


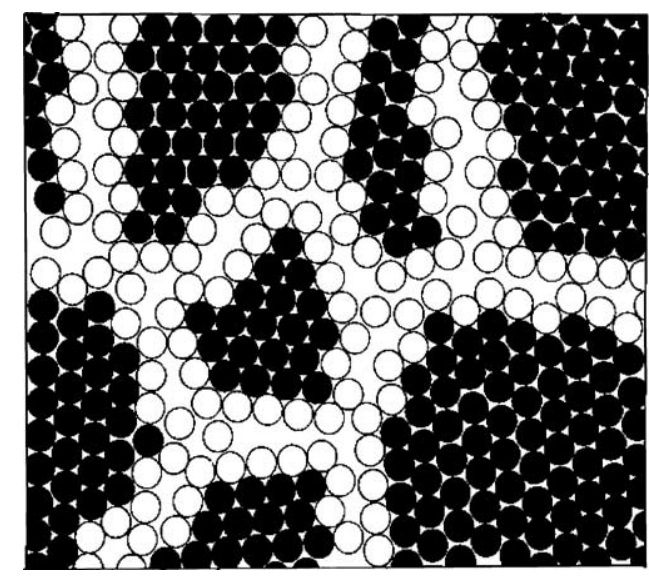

Fig. 7. Two-dimensional model of a nanocrystaline solid. The atoms in the center of the crystals are indicated in black. The ones in the boundary regions are represented as open circles (reprinted from [1] with permission from Elsevier).

reported that the grain boundaries of nanocrystalline pure Fe exhibiting a strongly reduced local atomic short-range order. This implies that the atomic structure in grain boundaries of nanocrystalline materials is different from that in conventional coarse-grained polycrystals [30]. Gleiter proposed a 'hard-sphere' two-dimensional model for a nanocrystalline solid as shown in Fig. 7 [28]. Two different structures of atoms are illustrated in the diagram: crystal atoms with neighbor configuration corresponding to the lattice and boundary atoms with a wide variety of interatomic spacings. In the boundary regions, the coordination between nearest neighbor atoms deviates or reduces from the one in the crystallites, implying the occurrence of atomic disorder at these regions [28,34]. Further, Gleiter demonstrated that several factors like size effects, changes of the atomic structure, and alloying elements could affect the properties of nanocrystalline materials. The possible effect of alloying elements on the atomic arrangements of nanocrystalline alloys is illustrated in Figs. 8 and 9. Solute atoms with little solubility in the lattice of the crystallites often segregate to the boundary regions [1]. In certain cases, the formation of a Ag-Fe alloy solid solution is favored even though the constituents are immiscible in the crystalline and molten state (e.g. Fe and Ag) [32].

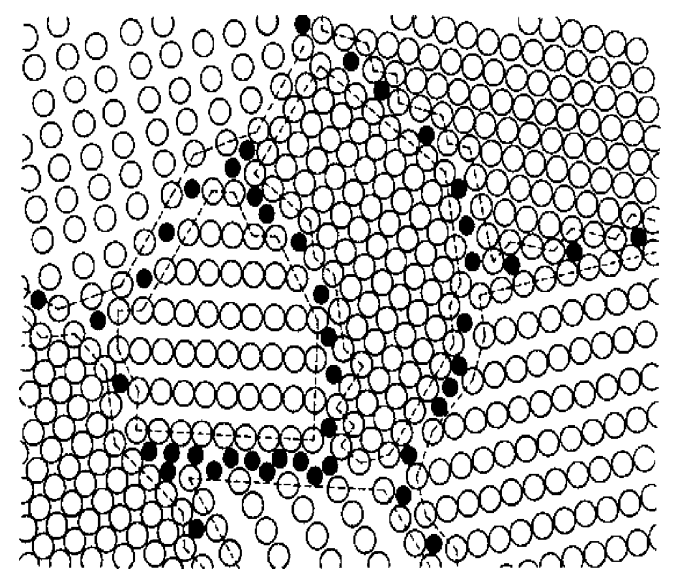

Fig. 8. Schematic model of the structure of the nanocrystalline $\mathrm{Cu}-\mathrm{Bi}$ and $\mathrm{W}-\mathrm{Ga}$ alloys. The open circles represent the $\mathrm{Cu}$ or $\mathrm{W}$ atoms, respectively, forming the nanometer-sized crystals. The black circles are the $\mathrm{Bi}$ or $\mathrm{Ga}$ atoms, respectively, incorporated in the boundaries of alloys (reprinted from [1] with permission from Elsevier). 


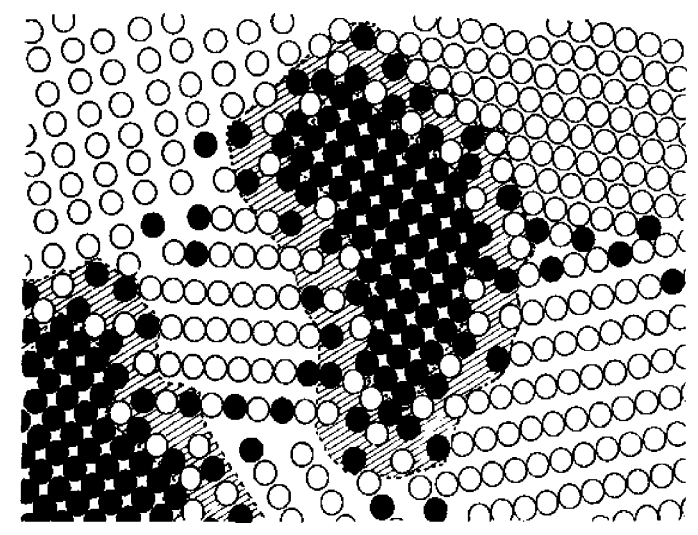

Fig. 9. Schematic model of nanocrystalline Ag-Fe alloys that consist of a mixture of nanometer-sized Ag and Fe crystals (represented by open and full circles, respectively). In the interfacial regions between $\mathrm{Ag}$ and $\mathrm{Fe}$ crystals, solid solution of Fe atoms in $\mathrm{Ag}$ crystallites, and $\mathrm{Ag}$ atoms in the Fe crystallites are formed although both component are immiscible in the liquid as well in the molten stat (reprinted from [32] with permission from Elsevier).

Nanocrystalline materials can be classified into several groups according to their dimensionality: zero-dimensional atom clusters, one-dimensional modulated multilayers, two-dimensional ultra-finegrained overlayers, and three-dimensional nanocrystalline structures (Fig. 10) [35]. The nanocrystalline materials may contain crystalline, quasi-crystalline and amorphous (nanoglasses) phases. They can be metals, intermetallics, ceramics and composites. Gleiter [36,37] classified nanocrystalline solids into twelve groups according to the shape (dimensionality) and chemical composition of their constituent structural elements (Fig. 11). According to the shape of crystallites, three categories of nanocrystalline materials may be distinguished: layer-shaped crystallites, rod-shaped crystallites (with layer thickness or rod diameters on the order of a few nanometers) and nanocrystalline materials composed of equiaxed nanometer-sized crystallites. Depending on the chemical composition of the crystallites, the three categories of nanocrystalline materials may be grouped into four families. In the first family, all crystallites and interfacial regions have the same chemical composition. The second

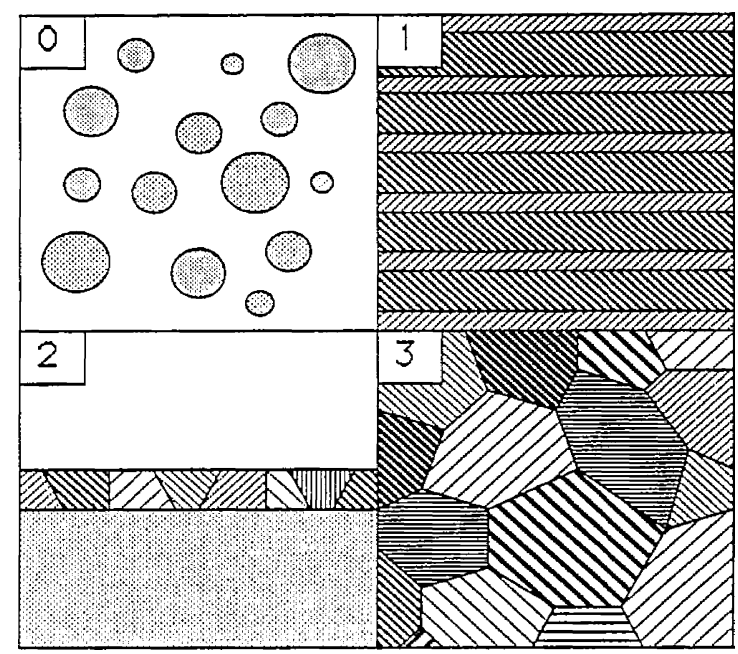

Fig. 10. Schematic of the four types of nanocrystalline materials according to Siegel (reprinted from [35] with permission from Elsevier). 


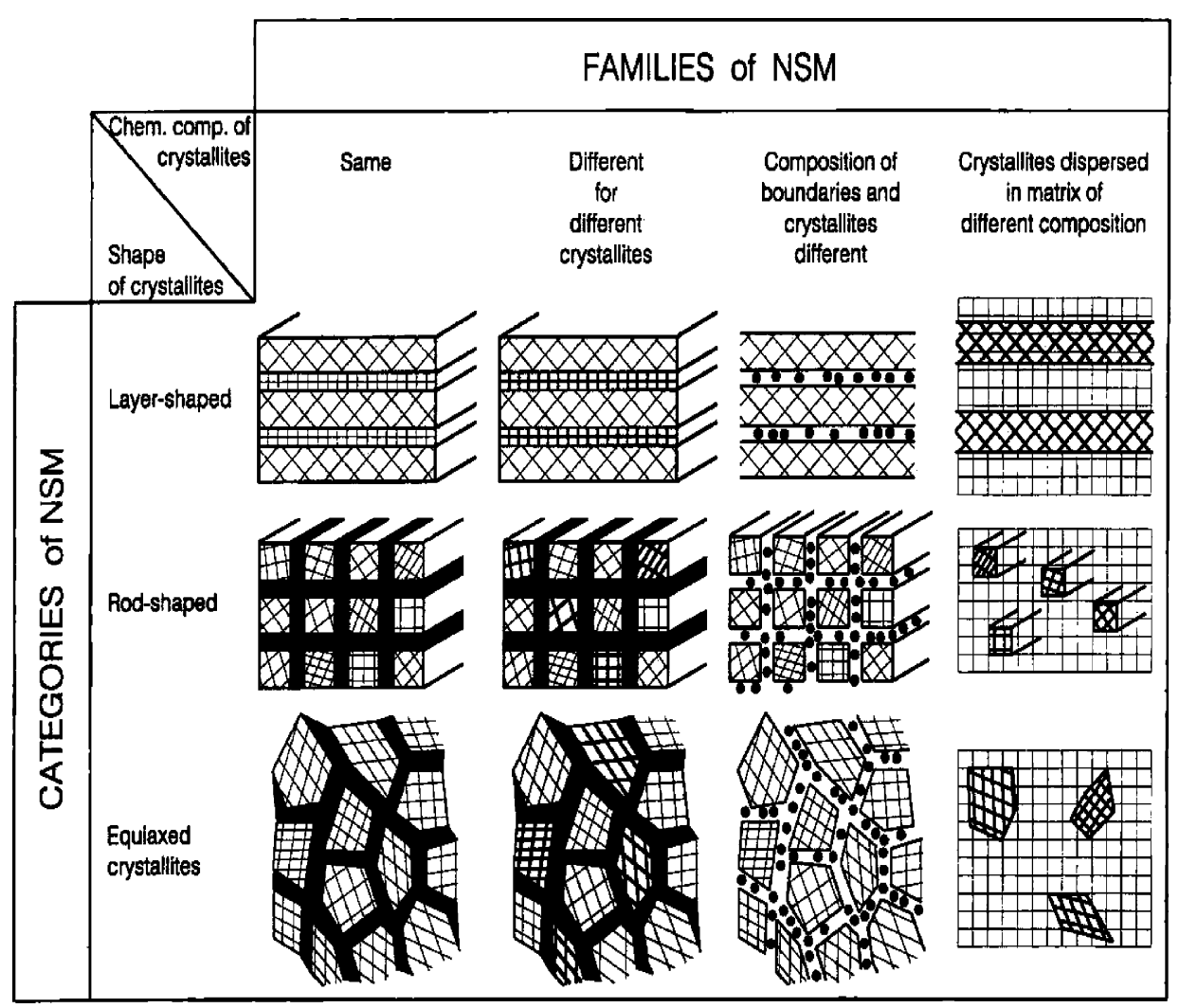

Fig. 11. Classification schema for nanocrystalline materials according to their chemical composition and the dimensionality (shape) of the crystallites (structural elements) forming the materials (reprinted from [37] with permission from Elsevier).

family consists of crystallites with different chemical compositions. If the composition variation occurs primarily between crystallite and the interfacial regions, the third family is obtained. In this case, one type of atoms tends to segregate preferentially to the interfacial regions (see also Fig. 8). The fourth family is formed by nanometer-sized crystallites (layers, rods, equiaxed crystallites) dispersed in a matrix of different chemical composition. Precipitation alloy, e.g. dispersion of $\mathrm{Ni}_{3} \mathrm{Al}$ in $\mathrm{Ni}$ matrix is a typical example of this family.

Grain growth is a crucial aspect of the thermal stability of nanocrystaline solids. Nanocrystalline materials are thermodynamically unstable due to the presence of a large fraction of interface boundaries. There is a strong tendency for nanocrystalline materials to convert to conventional coarser grained materials with fewer interfaces. Therefore, stabilization of the nanocrystalline grain structure is of critical importance for retaining their unique structures and properties. For conventional polycrystalline materials, the driving force for grain growth results from the the reductions of free energy of the system by decreasing the total grain boundary energy. The kinetic equation of grain growth can be described by the following equation:

$$
d^{2}-d_{0}^{2}=k t
$$

where $d$ is the average grain size after annealing, $d_{0}$ the initial grain size, $t$ the annealing time, and $k$ a material constant. However, the parabolic grain growth as expressed in Eq. (E1) is rarely observed except for high purity metals at high homologous temperatures. In most cases, the variation of grain 
growth as a function of temperature can be described by,

$$
d^{1 / n}-d_{0}^{1 / n}=k t
$$

where $n$ is the grain growth exponent (typically $n \leq 0.5$ ). $k$ is sensitive to the temperature of annealing, and can be expressed in an Arrhenius type equation:

$$
k=k_{0} \exp \left(-\frac{Q}{R T}\right)
$$

where $Q$ is the activation energy for grain growth and $R$ the gas constant. The activation energy $Q$ is commonly used to imply the microscopic mechanism which dominates the grain growth. The deviation from known parabolic relationship (i.e. $n=0.5$ ) is related to many factors such as pores and impurity doping, solute drag effect on the grain boundary migration, temperature change of the grain growth mechanism, initial grain size distribution, etc $[38,39]$. In the case of nanocrystalline materials, the mechanisms that are responsible for the enhanced thermal stability against grain growth are: (a) solute drag, (b) grain boundary segregation, (c) Zener pinning by secondary phases and (d) chemical ordering [40]. Accordingly, the activation energy $(Q)$ values for isothermal grain growth have also a wide dispersion. Most nanocrystalline metals have activation energy for grain growth closer to the respective grain boundary diffucion. For example, Iordache et al. have studied the grain growth behavior of pure nanocrystalline $\mathrm{Ni}$ prepared by electrodeposition between 603 and $683 \mathrm{~K}$ for various periods of time [41]. The activation energy for grain growth in nano-Ni is determined to be $102 \mathrm{~kJ} / \mathrm{mol}$, which is similar to that for grain boundary diffusion in polycrystalline $\mathrm{Ni}(108 \mathrm{~kJ} / \mathrm{mol})$. However, an anomalous grain growth behavior is found in mechanically milled nanocrystalline $\mathrm{Fe}$ [40]. The grain growth over the entire range of temperature studied cannot be described by a single, thermally activated rate process. The $Q$ values for nanocrystalline Fe in two intervals of annealing temperature $(T<725 \mathrm{~K}$ and $T>805 \mathrm{~K})$ are determined to be 125 and $248 \mathrm{~kJ} / \mathrm{mol}$, respectively. The former value is lower than the activation energies for grain boundary diffusion (174 kJ/mol) and the lattice sef diffusion in Fe $(251-282 \mathrm{~kJ} / \mathrm{mol})$. At higher annealing temperatures, the grain growth of nanocrystalline Fe is determined from Burke equation with respect to pinning forces causing stagnation [42]:

$$
\frac{d_{0}-d}{d_{\mathrm{m}}}+\ln \left[\frac{d_{\mathrm{m}}-d_{0}}{d_{\mathrm{m}}-d}\right]=k t
$$

where $d_{\mathrm{m}}$ is the maximum grain size that results from the pinning force. The activation energy for grain growth of $248 \mathrm{~kJ} / \mathrm{mol}$ is close to the activation energy for lattice diffusion. They attributed this behavior to the impurity-drag controlled grain boundary migration as the concentration of impurities along grain boundaries increases during the grain growth process. Therefore, the impurities may give rise to a retarding force that is dependent on grain growth process. Similar results were also found in mechanically milled nanocrystalline Al [43]. From the calculation of activation energy, Lavernia and coworkers also reported that the grain growth kinectic behavior of nanocrystalline $\mathrm{Al}$ is governed by two different mechanisms. The growth at lower temperatures $(T<723 \mathrm{~K})$ exhibits an apparent activation energy $(79 \mathrm{~kJ} / \mathrm{mol})$ close to that for grain boundary self-diffusion in polycrystalline $\mathrm{Al}$ $(84 \mathrm{~kJ} / \mathrm{mol})$. At higher temperatures $(T>723 \mathrm{~K})$, it shows a much higher activation energy $(112 \mathrm{~kJ} /$ $\mathrm{mol})$ close to that for lattice diffusion $(124 \mathrm{~kJ} / \mathrm{mol})$ on the basis of Burke analysis [43]. It is likely that the mechanical milling can induce various impurities in nanocrystalline $\mathrm{Fe}$ and $\mathrm{Al}$, thus the solute dependent grain growth predominates at higher temperature regime. The impurities in nanocrystalline Fe could be interstitial elements e.g. $\mathrm{O}, \mathrm{N}$ and substitutional solutes, like $\mathrm{Cr}$ and $\mathrm{Mn}$. And $\mathrm{Cr}$ is suggested to the main impurities responsible for inhibiting the grain boundary migration [40]. In the 
case of nanocrystalline $\mathrm{Al}$, the main impurities are $\mathrm{O}, \mathrm{N}, \mathrm{Fe}, \mathrm{Cr}$ and $\mathrm{Mn}$; and $\mathrm{Fe}$ is main solute that influences the grain boundary mobility [43]. At low temperature regime, the impurities are not activated and the nanocrystalline samples behave as pure materials. In this case, the activation for the grain growth is comparable to the activation energy for self-diffusion at the grain boundaries. At higher temperature regime, the grain growth is solute dependent, leading to impurity-drag controlled grain boundary migration. Therefore, the activation energy for the grain growth in nanocrystalline metals is close to the activation energy for lattice diffusion.

Differential scanning calorimetry (DSC) is commonly used to detect the release of interfacial grain boundary enthalpy during the grain growth process [40-46]. For nanocrystalline metals, (e.g. $\mathrm{Cu}$, $\mathrm{Pd}, \mathrm{Ag}$ ) prepared by inert gas condensation, the nano-sized grains tend to coarsen very rapidly even at room temperature [42] or at temperatures below the crystallization temperature [47]. Moreover, gaseous impurities (oxygen) tend to stabilize the grain boundaries of such elemental metal powder compacts [42]. However, non-uniform segregation of impurities and non-uniformity of the grain size distribution can result in large room-temperature grain growth in nanocrystalline $\mathrm{Cu}$ [47]. The reduction of impurities in metal powder compacts via improvement in the synthesis and compaction could lead to abnormal grain growth [48]. In the case of nanocrystalline metals prepared by electrodeposition, for example, the thermal stability against the grain growth is rather low. DSC and TEM results showed that the nonograins of electroplated $\mathrm{Ni}$ were stable up to $80{ }^{\circ} \mathrm{C}$, but began to grow abnormally in the range of $80-289^{\circ} \mathrm{C}$ [45]. Lavernia and coworkers studied the grain growth kinetics of electroplated $\mathrm{Ni}$ with a grain size of $20 \mathrm{~nm}$ through isothermal annealing at $330-410{ }^{\circ} \mathrm{C}$ [49]. They reported that a very rapid grain growth occurs in the very early stage of annealing and the growth returns to normal when the grain size exceeds $600 \mathrm{~nm}$. The activation energy for grain growth in nano-Ni was found to be $102 \mathrm{~kJ} / \mathrm{mol}$. This is similar to that for grain boundary diffusion in polycrystalline $\mathrm{Ni}(108 \mathrm{~kJ} / \mathrm{mol})$. Grain growth of electroplated nanocrystalline $\mathrm{Cu}$ occurred at about $70{ }^{\circ} \mathrm{C}$ [50]. To retard the grain growth of nanocrystalline $\mathrm{Cu}$, mechanical alloying of $\mathrm{Cu}$ with $\mathrm{Fe}$ to yield supersaturated metastable $\mathrm{Fe}-\mathrm{Cu}$ alloys has been proven to be an effective means. An improved thermal stability of single-phase fcc $\mathrm{Fe}-\mathrm{Cu}$ alloys resulted from solute segregation to nanocrystalline grain boundaries [51]. Electroplated nanocrystalline Ni doped with carbon or boron exhibit limited grain growth [52,53]. Suryanarayanan et al. also reported that B doping is beneficial to the retardation of grain growth in nanocrystalline $\mathrm{Cu}$ powders during densification and heat treatments [54]. In some cases, cryogenic reaction milling in liquid nitrogen has been reported to produce nanocrystalline $\mathrm{Al}$ and aluminum alloys with enhanced thermal stability [55,56]. Disko et al. suggested nanometer-scale particles of oxynitrides impeded the grain growth of the cryomilled $\mathrm{Al}$ and its alloys [55]. Lavernia and coworkers attributed the observed high grain size stability of Al Alloy to Zener pinning of the grain boundaries by $\mathrm{AlN}$ and $\mathrm{Al}_{2} \mathrm{O}_{3}$ particles and solute dragging of numerous atomic species. Such particles are formed in the metal powders during cryomilling [56].

In evaluating thermal stability and mechanical properties of nanocrystalline materials, grain size and internal strain are very important factors. The X-ray diffraction (XRD) profile is a useful tool for the characterization of microstructures of nanocrystalline solids in which small crystallite size and lattice microstrain can cause broadening of the diffraction peaks. The changes in width and shift of XRD profiles are analyzed accordingly. It is generally known that TEM can provide a direct image of the grain size distribution. However, TEM imaging is localized within a small region of the sample and the preparation procedures of TEM samples are tedious. Further, grain overlap poses a serious problem. The advantages of XRD over TEM for grain size measurement are obvious, i.e. average grain size is determined from larger area of sample and ease of sample preparation. Several theoretical models have been developed to obtain structural information through mathematical analysis of the XRD profile [57-60]. The applicability of the XRD line profile analysis in determining the grain size 
and microstrain in nanocrystalline materials has been conducted extensively by the researchers [40,57-67]. In general, the experimental peak profile is made up of the instrumental profile and the intrinsic profile. The simplified method for substracting the effect of instrumental broadening is based on the assumptions that the instrumental (reference) profile and the intrinsic profile can be approximated by the Cauchy (Lorentz) or Gaussian $(\mathrm{G})$ peak shape function. Generally, the integral width $(\beta)$ can be described using the Cauchy-Cauchy (CC), Gaussian-Gaussian (GG) or CauchyGaussian relationship [57]. $\beta$ is the broadening or full-width of the diffraction line measured at half maximum intensity (FWHM). Such relationships can be expressed as:

$$
\begin{aligned}
& \beta_{\text {exp }}=\beta+\beta_{\text {inst }} \quad(\mathrm{CC}) \\
& \beta_{\text {exp }}^{2}=\beta^{2}+\beta_{\text {inst }}^{2} \quad(\mathrm{GG}) \\
& \left(\frac{\beta}{\beta_{\text {exp }}}\right)=1-\left(\frac{\beta_{\text {inst }}}{\beta_{\text {exp }}}\right)^{2}
\end{aligned}
$$

where $\beta_{\text {exp }}, \beta$ and $\beta_{\text {inst }}$ are the integral breadth of experimental profile, true diffraction (intrinsic) profile and instrumental profile, respectively. Instrumental broadening corrections are typically made with a large grained and strain-free standard sample.

The GG relation (Eq. (E6)) is widely employed in most routine calculations for the determination of the intrinsic profile on the basis that the peak shapes are Gaussian [57,61]. After subtracting the instrumental broadening, the small grain size and/or microstrain contribution to intrinsic broadening of Bragg refelection peaks can be determined. In the absence of microstrain, the grain size of powder is determined from the Scherrer equation [58]:

$$
\beta=\frac{0.9 \lambda}{L \cos \theta}
$$

where $\lambda$ is the $\mathrm{X}$-ray wavelength, $L$ is the average dimensions of crystallites and $\theta$ is the Bragg angle. It is often to express the reflection breadth in $\delta s$ units $[s=2(\sin \theta) / \lambda]$ as follows:

$$
L=\frac{K}{[2 \cos \vartheta / \lambda] \delta \vartheta}=\frac{K}{\delta s}
$$

Determination of the grain size by the Scherrer equation does not accout for the internal lattice strain that can also cause broadening of the diffraction peaks. There is a tendency for underestimation of the grain size due to the ignorance of microstrain effect, particularly for sample prepared via cold-pressing that containing internal strain [61]. In the presence of size and strain broadening, several approaches can be used to determine the size and microtrain of particles from intrinsic profile using $\mathrm{CC}, \mathrm{GG}$ or $\mathrm{CG}$ relations [57]:

$$
\begin{aligned}
& (\delta s)=(\delta s)^{L}+(\delta s)^{S} \quad(\mathrm{CC}) \\
& (\delta s)^{2}=\left[(\delta s)^{L}\right]^{2}+\left[(\delta s)^{S}\right]^{2} \quad(\mathrm{GG})
\end{aligned}
$$

where the superscript $L$ and $S$ refer to the size and strain effect, respectively. The CC approach is generally referred to as Williamson and Hall procedure [59]. In terms of $\beta$ expression, it can be 
described as:

$$
\begin{aligned}
& \beta=\frac{0.9 \lambda}{d \cos \theta}+2 \varepsilon \tan \theta \\
& \beta \cos \theta=0.9(\lambda / d)+2 \varepsilon \sin \theta
\end{aligned}
$$

where $\varepsilon$ is the lattice microstrain. If the $\beta \cos \theta$ is plotted against $\sin \theta$ for a number of XRD peaks at different angles, the data should fall on a straight line, with a slope of $(2 \varepsilon)$ and an intercept of $(0.9 \lambda / d)$. The CC approach is inappropriate to use because it yields sizes that are much larger than those determined from other methods. It also produces inconsistent results for the grain size. The validity of Eq. (E12) requires the sample to have inherent isotropic property in all crystallographic directions. Various reflection peaks must be used to minimize the measurement error. However, Malow and Koch

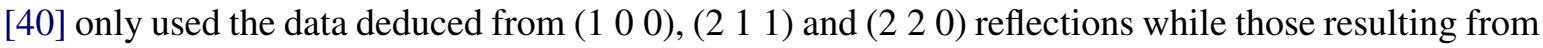
$\left(\begin{array}{lll}1 & 1 & 0\end{array}\right)$ and $\left(\begin{array}{lll}3 & 1 & 0\end{array}\right)$ reflections are excluded in the determination of grain size of nanocrystalline $\mathrm{Fe}$ using Eq. (E12). This is due to the elastic modulus on ( $\left.\begin{array}{lll}1 & 1 & 0\end{array}\right)$ and $\left(\begin{array}{lll}3 & 1 & 0\end{array}\right)$ planes differ significantly from those on the other three cry stall ographic planes. This leads to different microstrains in $\left\langle\begin{array}{lll}1 & 1 & 0\end{array}\right\rangle$ and $\left\langle\begin{array}{lll}3 & 1 & 0\end{array}\right\rangle$ directions relative to those in other three crystallographic direactions. Consequently, all the five data points will not lie on a straight line. Klug and Alexander [57] suggested that it is more appropriate to fit the strain broadening with a Gaussian function and to represent the size broadening with a Cauchy function (CG relation). Several workers have reported that the CG relation for intrinsic profile deconvolution provides reasonable results for cold-pressed nanocrystalline $\mathrm{Al}$ samples and strained $\mathrm{Cu}$ specimens $[61,62]$.

The CG relation is given by:

$$
\frac{\beta^{2}}{\tan ^{2} \theta_{0}}=\frac{K \lambda}{d}\left(\frac{\beta}{\tan \theta_{0} \sin \theta_{0}}\right)+16 \varepsilon^{2}
$$

where $\theta_{0}$ is the position of peak maximum. By plotting $\beta^{2} / \tan ^{2} \theta_{0}$ against $\left(\beta / \tan \theta_{0} \sin \theta_{0}\right)$, a linear relationship can be obtained from the least square analysis. The grain size and microstrain are determined from the slope $K \lambda / d$ and ordinate intercept $16 \varepsilon^{2}$, respectively.

\section{Synthesis of nanoparticles and nanorods/nanotubes}

Significant progress has been made over the last decade in understanding fundamental aspects of the synthesis of nanoparticles, nanorods, etc. Various processing routes have been developed for the synthesis and commercial production of nanoparticles including vapor, liquid solid state processing routes, and combined method, such as vapor-solid-liquid approach (Table 2). The most common method used for the synthesis of metal and ceramic nanoparticles with a well-defined and narrow size distribution is inert-gas condensation. Different sources such as evaporation, sputtering and laser have been adopted to vaporize materials from the surface of solids into clusters. The clusters generated are condensed, transported to and collected on a cold finger. The nanoparticles formed are then in-situ compacted under high-vacuum conditions to form a clean bulk nanocrystalline solid. Chemical vapor deposition (CVD) involves the creation of vapor species through the decomposition of gaseous reactants in the vicinity of a hot substrate to form solid films, nanoparticles, nanorods or nanotubes. Thermal, laser or plasma energies are used for the decomposition of gaseous reactants. CVD is a wellestablished technique for thin film deposition for many years because it offers the advantages of a 
Table 2

Classification of available techniques to synthesize nanocrystalline particles and nanotubes

\begin{tabular}{ll}
\hline Route & Processing \\
\hline Vapor & Physical vapor deposition, chemical vapor deposition, aerosol processing \\
Liquid & Sol-gel process, wet chemical synthesis \\
Solid & Mechanical alloying/milling, mechanochemical synthesis \\
Combined & Vapor-liquid-solid approach \\
\hline
\end{tabular}

relatively simple apparatus, excellent film uniformity, high film density, high deposition rates and amenability to large-scale production. It is also a promising tool for synthesizing nanotubes and nanoparticles. However, the use of flammable, corrosive or toxic reactant gases may pose problems in laboratory safety and personnel health. Aerosol processes have several distinct advantages such as single step processing, versatility and high production rate. In these processes, the liquid precursors are converted to the vapor phase followed by condensation into droplets. These methods may be used to produce a variety of ceramic powders owing to the availability of a wide range of precursors.

High-purity, homogeneous and nanosized ceramic particles can also be prepared via liquid routes. Sol-gel processing is particularly attractive because the chemical reactions can be tailored at the molecular level. Using molecular precursors to obtain an oxide or hydroxide network via hydrolysis and condensation reactions, sol-gel process provides new approaches and a better control in the preparation of nanomaterials. However, the alkoxides used are rather expensive, and the processing is tedious because it requires several sequential steps to form nanoparticles or nanocrystalline solids.

The above-mentioned synthesized techniques involve the assembly of cluster sof atoms in the vapor or liquid phase and its subsequent condensation to form nanoparticles. In contrast, solid state route such as mechanical alloying/milling produces its nanostructures not by cluster assembly but rather through the structural decomposition of coarser grained structures as the result of severe plastic deformation. It is potentially method of producing powders with a broad range of chemical composition and structure from the amorphous, intermetallic, supersaturated solid solution to nanocrystalline phases. The main problems of mechanical alloying are contamination and grain growth of the powders during processing.

\subsection{Vapor route}

\subsubsection{Physical vapor deposition}

Physical vapor deposition (PVD) is a versatile synthesis method and capable of preparing thin film materials with structural control at the atomic or nanometer scale by careful monitoring the processing conditions. PVD involves the generation of vapor phase species either via evaporation, sputtering, laser ablation or ion beam. In evaporation, atoms are removed from the source by thermal or electron means; in sputtering, atoms are ejected from the target surface by the impact of energetic ions. In the former case, the vapor phase species that experience collisions and ionization are condensed onto a substrate follow by the nucleation and growth. Thermal evaporation has a limitation in multicomponent materials since one of the metallic elements typically evaporates before the other due to the differences in vapor pressures of the evaporating species. On the contrary, sputtering is capable of depositing high melting point materials such as refractory metals and ceramics, which are difficult to fabricate using evaporation. Since the sputtered atoms carry more energy than the evaporated atoms, the sputter-grown films usually have higher density. Owing to the lower purity of the sputtering target materials, sputtered films are more prone to contamination than evaporated films. 
Inert gas condensation combined with thermal evaporation is commonly used to fabricate metallic and metal-oxide powders with a well defined and narrow size distribution $[9,17,30,68$ 74]. This technique was originally introduced by Ganqvist and Buhrman [75] in 1976 and developed by Gleiter in 1981 [76]. In the process, a metal is evaporated inside an ultrahigh vacuum (UHV) chamber filled with inert gas, typically helium (Fig. 12). Vapors from the hot sources migrate into a cooler gas by a combination of convective flows and diffusion. The vaporized species then lose energy via collisions with helium molecules. As collisions limit the mean free path, supersaturation can be achieved above the vapor source. At high supersaturation, the vapors rapidly nucleate, forming large numbers of clusters that grow via coalescence and agglomeration $[9,77,78]$. The clusters entrained in the condensing gas are transported to a vertical liquid nitrogen filled cold finger surface by a convection flow. The particles are removed from the cold finger by means of a scraper assembly. They are collected via a funnel and transported to an in-situ compaction device. Consolidation is performed first in the low-pressure compaction unit and then in the high-pressure compaction unit. The scraping

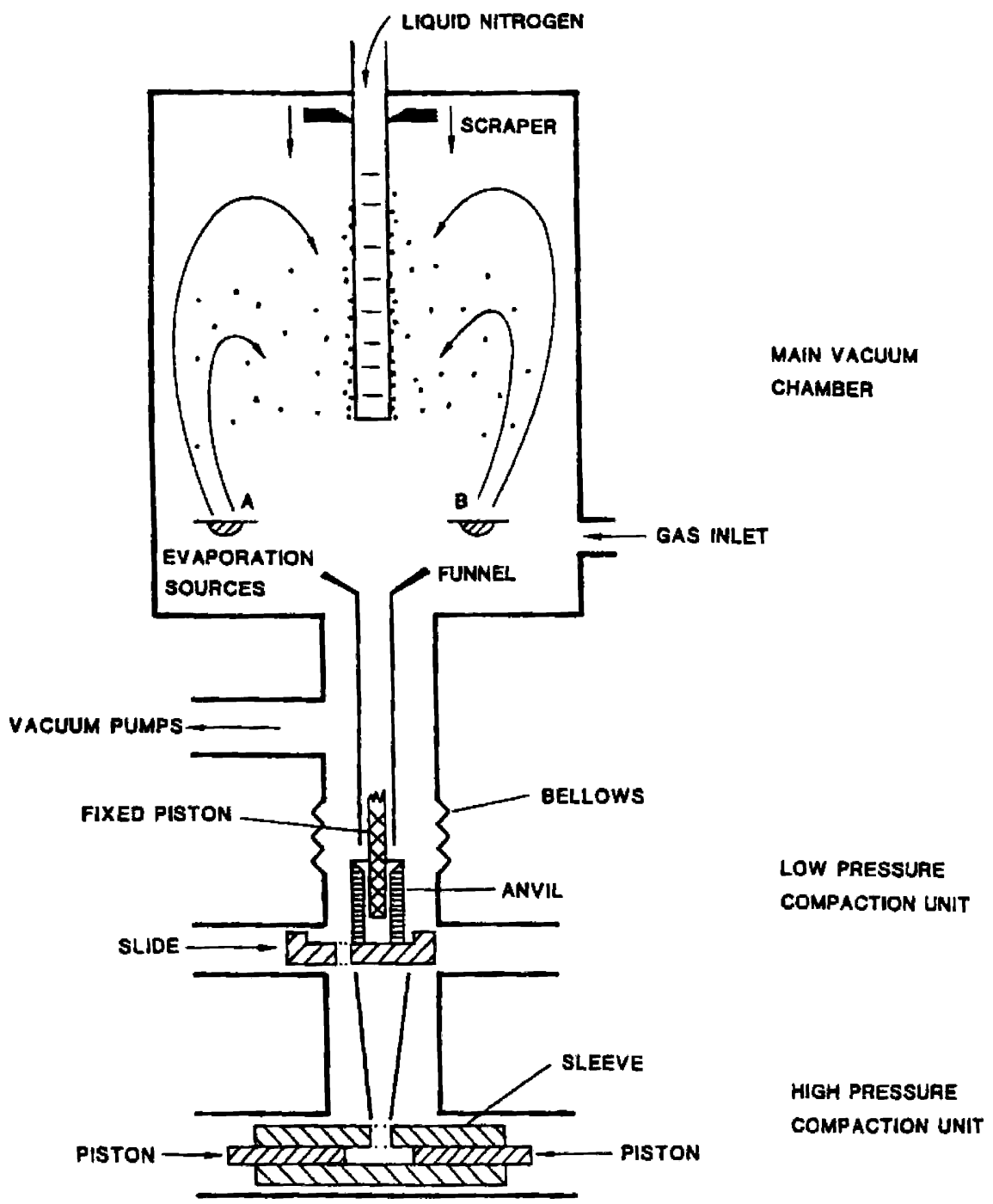

Fig. 12. Inert-gas condensation facility for the synthesis of nanocrystalline particles (reprinted from [1] with permission from Elsevier). 


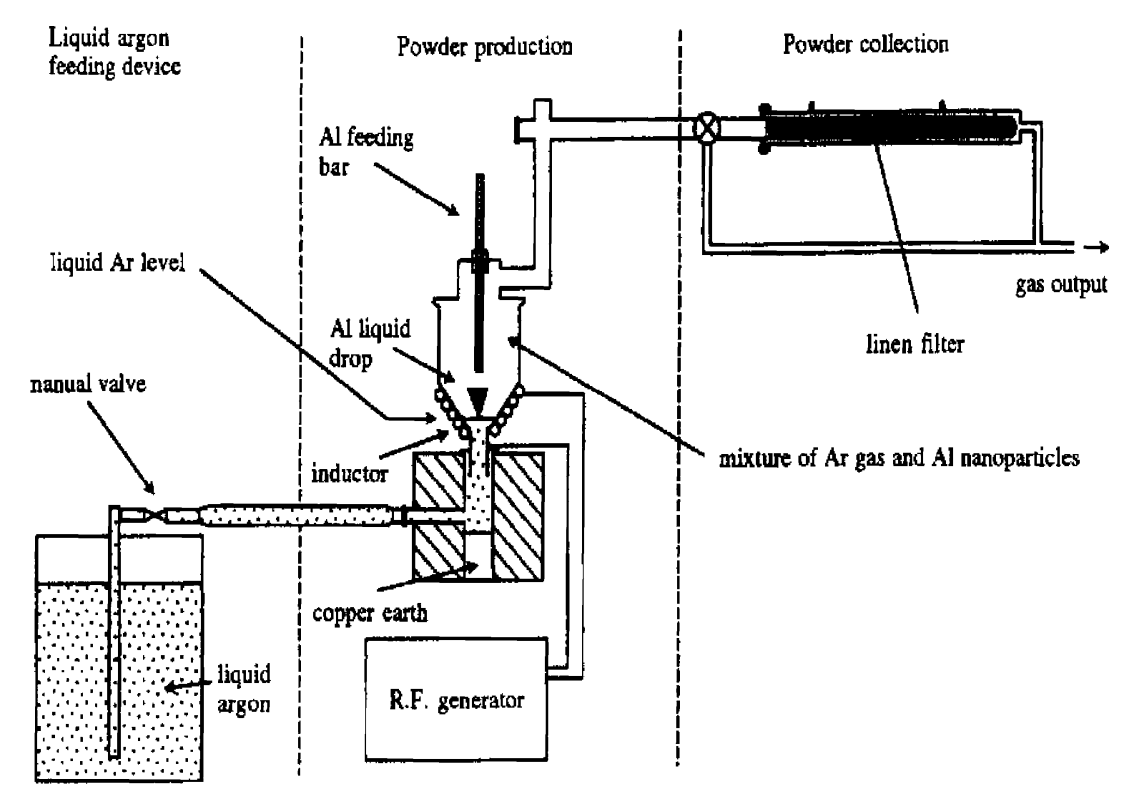

Fig. 13. Experimental se up for the synthesis of nanocrystalline aluminum powders via cryomelting (reprinted from [82] with permission from Elsevier).

and consolidation of particles are carried out under UHV conditions. Consolidation pressure of 1$2 \mathrm{GPa}$ is easily accomplished at room temperature [71]. The size, morphology and yield rate of the clusters in gas-condensation is dependent on three fundamental rates. They are: (1) the rate of supply of atoms to the region of supersaturation where condensation occurs, (2) the rate of energy removed from the hot atoms via the condensing gas medium, and (3) the rate of removal of clusters once nucleated from the supersaturated region [76]. Moreover, particle nucleation, coalescence (sintering) and growth during condensation also play key roles in forming small particles in large numbers [79].

As the yield of inert gas deposition is rather low, Bigot and coworkers developed a different process, i.e. cryogenic melting to produce metallic nanocrystals. The main advantage of this technique is that it exhibits a higher production rate of about $60 \mathrm{~g} \mathrm{~h}^{-1}$ and a yield of $75 \%$ [80-82]. Fig. 13 is a schematic diagram of the cryogenic melting apparatus [81]. The powders are produced by a spontaneous metallic gas condensation from a cryogenic medium. Rapid overheating of the metallic metal via radio frequency (RF) technique produces a substantial evaporation rate. Thus, RF induction enables the metal to reach a sufficiently high temperature to produce the high vapor pressure required. The reactor is supplied continuously with cryogenic liquid, and nanoparticles are formed by rapid condensation of the supersaturated metal vapor. The condensation region, where the particles are formed by the nucleation, growth and coalescence processes, is featured by a high temperature gradient, i.e. typically from $2200 \mathrm{~K}$ at the metallic surface but drop to $77 \mathrm{~K}$ in the cryogenic medium. The low temperature of the surrounding medium produces a high rate of nucleation and a rapid cooling of the as-formed particles with limited crystal growth [80-82]. Liquid argon is used for the synthesis of Al nanoparticles to prevent the formation of aluminum nitrides. However, liquid nitrogen is used for forming $\mathrm{Cu}$ or $\mathrm{Fe}$ nanopowders. This technique yields $\mathrm{Cu}$ nanoparticles having spherical shape with their size distribution following a log-normal function peaking at $25 \mathrm{~nm}$. The aluminum nanoparticles produced are also spherical having a size less than $70 \mathrm{~nm}[81,82]$. A schematic diagram showing the formation of $\mathrm{Al}$ nanoparticles associated with the condensation of metal vapor in cold argon gas flow is shown in Fig. 14. The growth of nanoparticles via coalescence and agglomeration is apparent in the diagram [82]. 


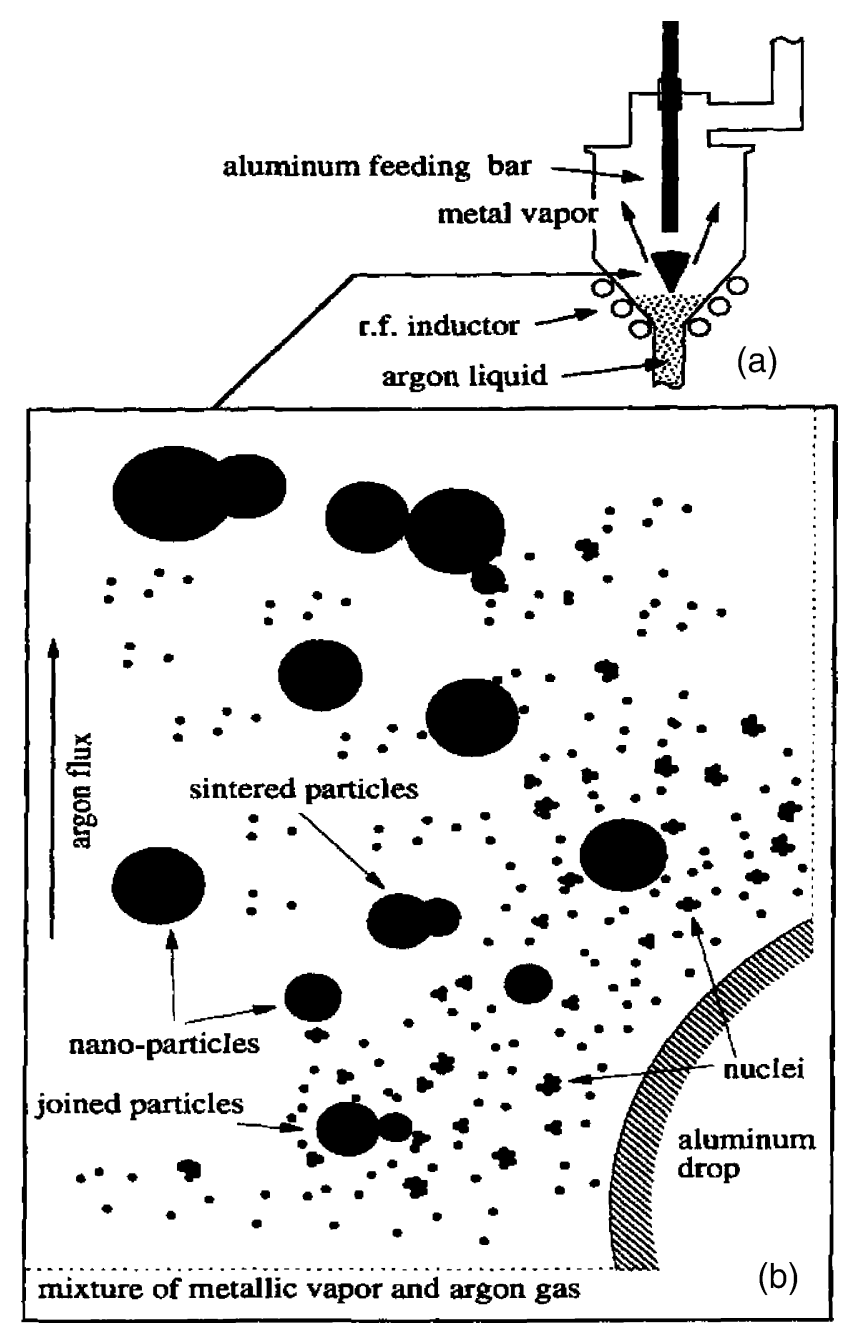

Fig. 14. Nucleation, coalescence and agglomeration of aluminum nanoparticles in argon gas during cryomelting process (reprinted from [82] with permission from Elsevier).

Inert gas condensation using a DC or RF magnetron sputtering source has been used to prepare refractory metal and ceramic nanoparticles [83-91]. The method of sputtering and gas condensation is crucially dependent on several processing parameters such as gas pressure, sputter power and sourcesubstrate distance. Such parameters must be selected carefully to achieve the formation of nanoparticles rather than the formation of a granular film [92]. Gonzalez et al. [90] used DC sputtering and gas condensation to obtain nanoparticles of Mo, MoNi and MoW. They reported that the particle size is strongly dependent on the argon pressure for all the materials prepared. The average particle size of Mo decreased from $12 \mathrm{~nm}$ at 0.4 mbar to $5 \mathrm{~nm}$ at 0.8 mbar. Fig. 15 shows the TEM micrographs and particle distribution of Mo, MoNi and MoW nanoparticles [90]. The morphology of Mo and MoNi formed at 0.2 mbar is self-arranged and produces well separated particles (Fig. 15a and f. In contrast, chain-like patterns of well-faceted cubes are observed for Mo formed at higher pressures because of coalescence during the formation process of the particles (Fig. 15b-d).

It is generally known that the laser ablation can provide better control of the evaporation process by congruent evaporation of constituent elements of multi-component materials in a very short period 
(a)
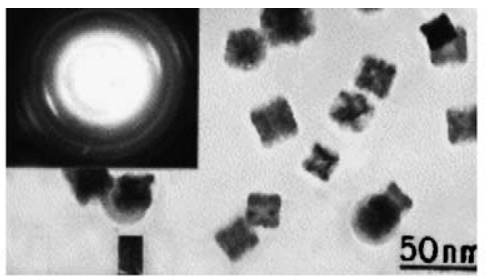

(b)

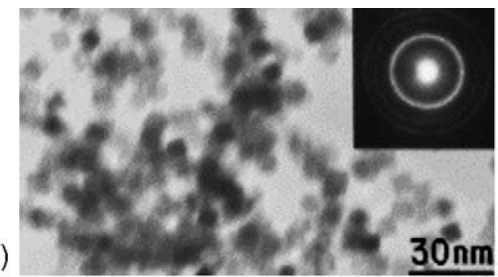

(c)

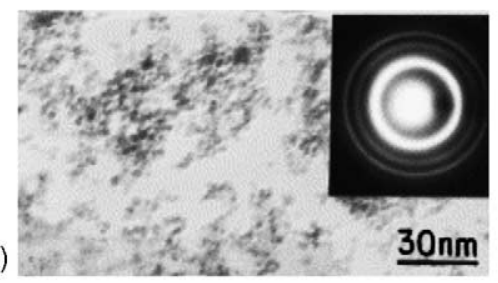

(e)

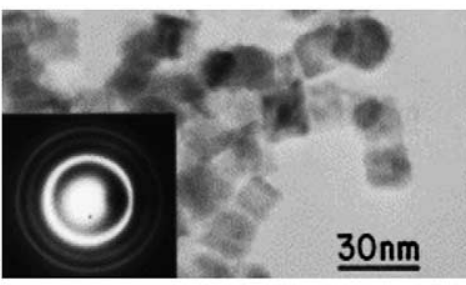

(g)

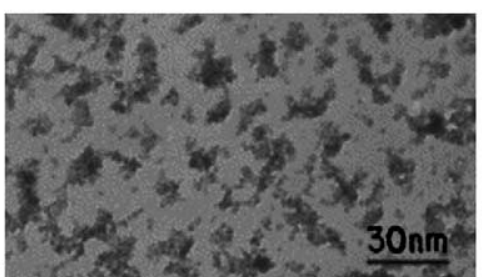

(d)

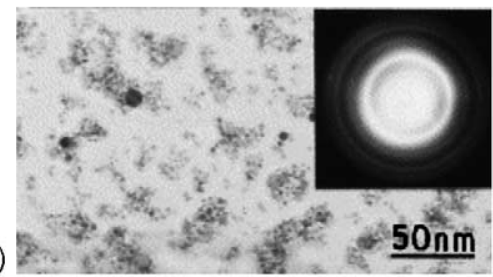

(f)

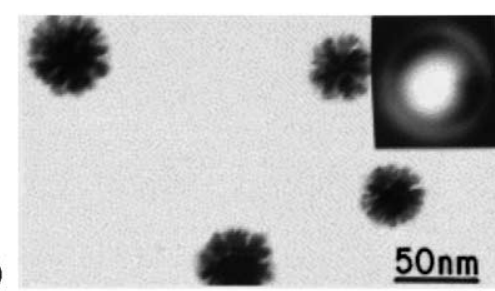

(h)

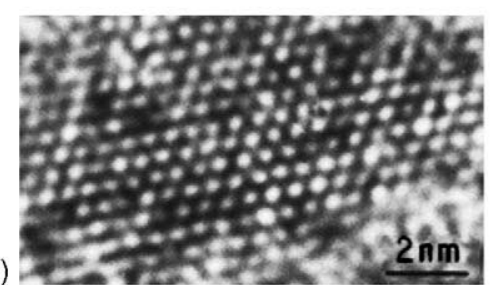

Fig. 15. TEM micrographs showing morphology, structure and particle distribution for Mo, MoNi and MoW formed by DC sputtering and gas condensation: (a) Mo at $0.2 \mathrm{mbar}$, (b) Mo at $0.4 \mathrm{mbar}$, (c) Mo at $0.6 \mathrm{mbar}$, (d) Mo at $0.8 \mathrm{mbar}$, (e) MoW at 0.4 mbar, (f) MoNi at $0.2 \mathrm{mbar}$, (g) MoNi at $0.8 \mathrm{mbar}$ and (h) HRTEM image of MoNi (0.8 mbar) (reprinted from [90] with permission from Elsevier).

of time. In this technique, an intense pulsed laser beam irradiates the target of interest, thereby vaporizing atoms and clusters from the target. The ablation rate is related to the total mass ablated from the target per laser pulse. Laser ablation in combination with inert gas condensation is an attractive route to synthesize larger amounts of the multicomponent nanocrystalline materials. Yamamoto and Mazumder adopted such an approach to synthesize $\mathrm{NbAl}_{3}$ intermetallic compound by using an excimer laser source. Their set-up is shown in Fig. 16. In the process, ablated atoms by laser pulse tend to collide with helium gas, losing their kinetic energy rapidly. They eventually condense to form a cloud consisting of fine nanocrystalline materials. The production rate varies with helium gas pressure and laser pulse energy [93]. Several workers then employed the laser ablation and gas condensation to produce nanoparticles of metals, metal oxides and metal carbides [94-99].

\subsubsection{Chemical vapor deposition}

Chemical vapor deposition (CVD) is a process where one or more gaseous adsorption species react or decompose on a hot surface to form stable solid products. The main steps that occur in the CVD process can be summarized as: 


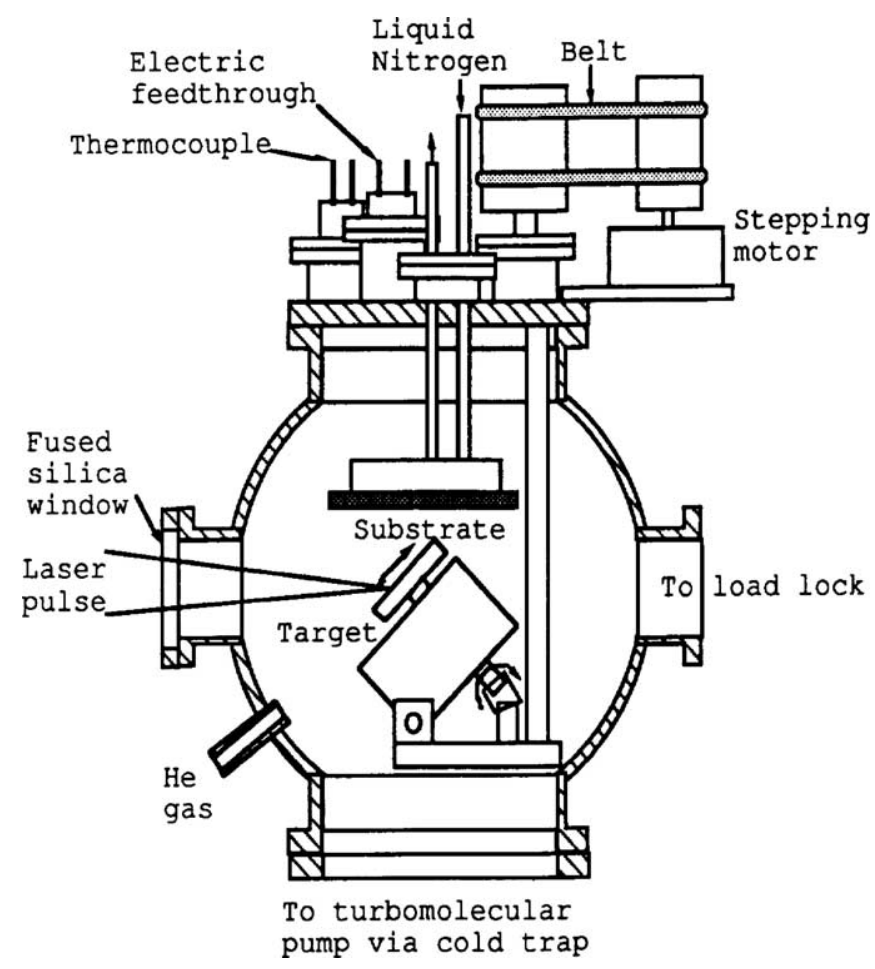

Fig. 16. Schematic drawing of laser ablation chamber equipped with a rotating target holder (reprinted from [93] with permission from Elsevier).

(a) Transport of reacting gaseous species to the surface.

(b) Adsorption of the species on the surface.

(c) Heterogeneous surface reaction catalyzed by the surface.

(d) Surface diffusion of the species to growth sites.

(e) Nucleation and growth of the film.

(f) Desorption of gaseous reaction products and transport of reaction products away from the surface $[100,101]$.

CVD is a more complex method of forming thin films and coatings than PVD. CVD exhibits several distinct advantages such as the capability of producing highly pure and dense films or fine particles at reasonably high deposition rates, and the capablility of coating complex-shaped components uniformly due to its non-line-of-sight nature. CVD is widely used for the deposition of metallic, ceramic and semiconducting thin films. Depending on the activation sources for the chemical reactions, the deposition process can be categorized into thermally activated, laser-assisted and plasma-assisted CVD.

In conventional thermally activated CVD, resistive heating of hot wall reactors provides sufficiently high temperatures for dissociation of gaseous species. This leads to the entire heating of the substrate to a high temperature before the desired reaction is achieved. It precludes the use of substrates having melting points much lower than the reaction temperature. Alternately, one could heat the reacting gases in the vicinity of the substrate by placing a hot tungsten filament inside the reactor. More recently, Lai et al. [102] employed hot filament CVD to grow one dimensional SiC nanorods. $\mathrm{SiC}$ nanorods are considered to be potential materials for use in structural and electronic applications because of their remarkable properties such as high hardness, high thermal stability, wide band gap and 


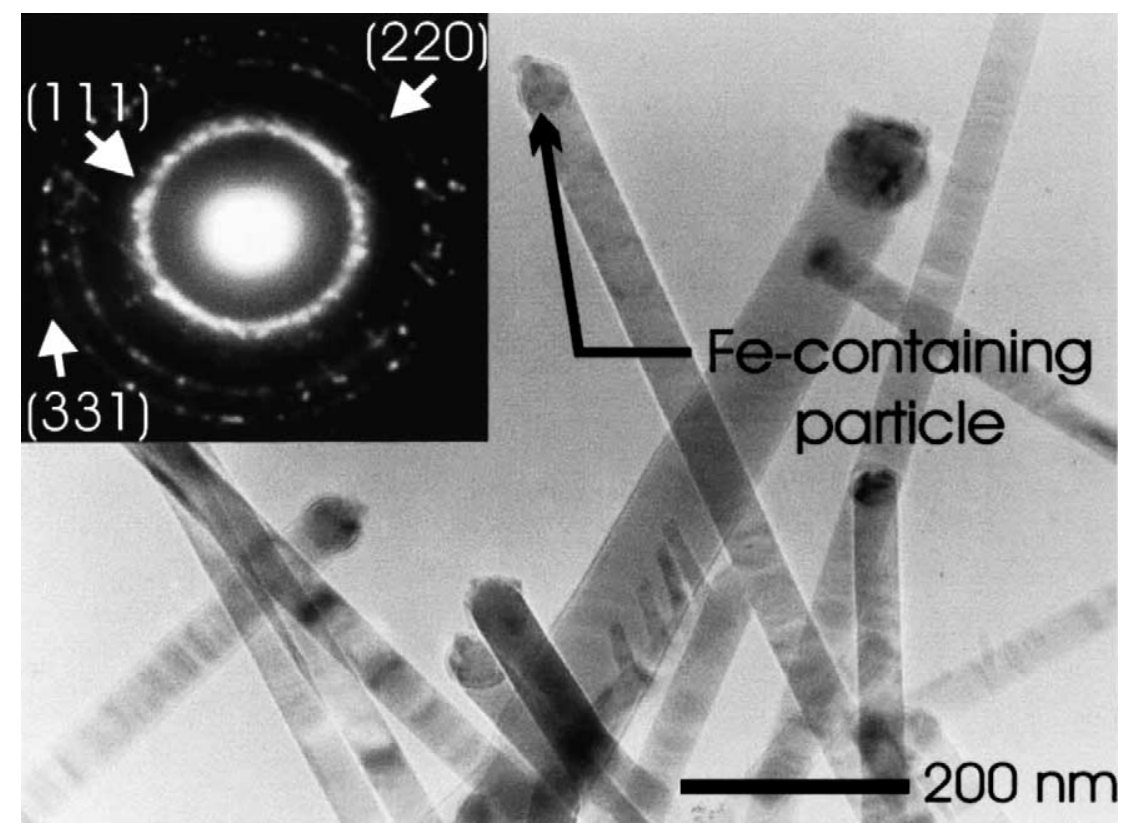

Fig. 17. Cross-sectional TEM micrograph and selected electron diffraction pattern of $\beta$-SiC nanorods (reprinted from [102] with permission from American Institute of Physics).

high electron mobility. Further, the Young's modulus of the nanorod can reach $\sim 600 \mathrm{GPa}$, which is

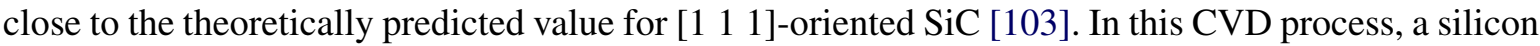
wafer was initially immersed in aqueous $\mathrm{Fe}\left(\mathrm{NO}_{3}\right)_{3}$ solution to remove the contaminants before transferring into the reactor chamber. The silicon and carbon sources were generated from a plate made of compacted carbon, silicon and silicon dioxide powders. The chamber was filled with hydrogen gas and tungsten filament was used to activate the hydrogen gas. The filament temperature was maintained at $2570 \mathrm{~K}$ whilst the substrate temperature was about $1373 \mathrm{~K}$. The hot filament dissociated molecular hydrogen to atomic hydrogen. The $\mathrm{Fe}\left(\mathrm{NO}_{3}\right)_{3}$ particles on the Si wafer surface were reduced by high concentration of atomic hydrogen to iron nanoparticles which acted as a catalyst. Further, the silicon and carbon sources were thermally activated by hot filaments to form hydrocarbon and silicon monoxide radicals. Fig. 17 shows the TEM image and selected electron diffraction pattern of $\beta-\mathrm{SiC}$ nanorods. The rods are straight with diameters of $20-70 \mathrm{~nm}$ and lengths of about $1 \mu \mathrm{m}$. Iron catalyst particles can be seen at the tips of nanorods. HRTEM images revealed that the nanorods grew along the $\left[\begin{array}{lll}1 & 0 & 0\end{array}\right]$ direction (Fig. 18). The current-voltage characteristics of nanorods follow the Fowler-Nordheim $(\mathrm{F}-\mathrm{N})$ relation. The turn-on field, which is defined as the electric field leading to a current density of $0.01 \mathrm{~mA} / \mathrm{cm}^{2}$, is $9 \mathrm{~V} / \mu \mathrm{m}$ [102]. According to the $\mathrm{F}-\mathrm{N}$ theory, the field-emission current density $I$, can be expressed as a function of the actual emission area of the emitter $A$, the applied electric field, the local work function of the emitter $\operatorname{tip} \varphi$, and a field-enhancing factor $\beta$. That is $I \sim$ $A\left(\beta^{2} / \varphi\right) E^{2} \exp \left[-7 \times 10^{7} \varphi^{3 / 2} / \beta E\right]$. For a field-emission phenomenon, the plot of $\ln \left(I / E^{2}\right)$ versus $\left(E^{-1}\right)$ should yield a straight line.

Plasma-enhanced CVD is known to exhibit a distinct advantage over thermal CVD owing to its lower deposition temperature. Various types of energy resources e.g. DC, RF, microwave and electron cyclotron resonance microwave (ECR-MW) radiation are currently used for plasma generation in CVD. In a DC plasma, the reacting gases are ionized and dissociated by an electrical discharge, generating a plasma consisting of electrons and ions. Microwave plasmaa are attractive because the 


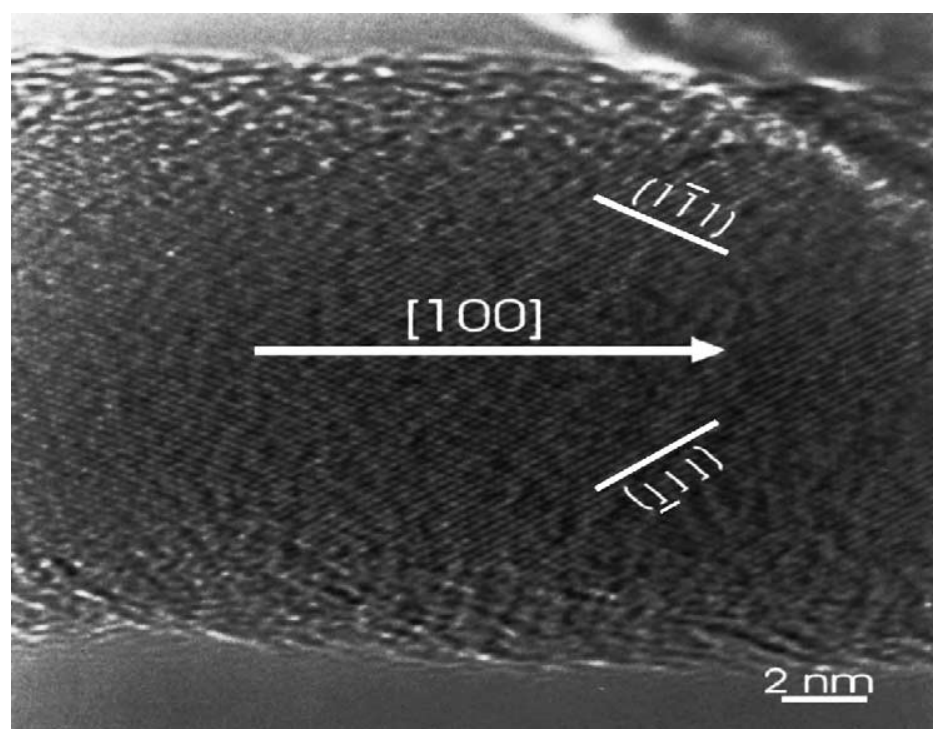

Fig. 18. HRTEM image of $\beta$-SiC nanorods (reprinted from [102] with permission from American Institute of Physics).

excitation microwave frequency $(2.45 \mathrm{GHz})$ can oscillate electrons. Thus, high ionization fractions are generated as electrons collide with gas atoms and molecules. SiC films have been deposited on silicon substrates by RF plasma-enhanced CVD using a gaseous mixture of $\mathrm{SiCl}_{4}, \mathrm{CH}_{4}, \mathrm{H}_{2}$ and $\mathrm{Ar}$ [104].

More recently, microwave plasma-enhanced CVD has been used to grow one dimensional carbon nanotubes at a low temperature range of $600-800{ }^{\circ} \mathrm{C}$. Well-aligned carbon nanotube arrays are required for cold cathodes in field emission displays. The MW-CVD method has the advantages of producing well-alignment and selective growth on various substrates [105,106]. Carbon nanotubes have attracted an increasing attention from materials scientists due to their unique shapes, superior field emission properties, excellent thermal conductivity, extraordinary high stiffness and axial strength. The novel structures could have potential applications as building blocks of nanoscale electronic devices and sensors [107]. Due to their large aspect ratios and small diameters, carbon nanotubes have emerged as potentially attractive materials as reinforcing elements in polymer and metal-matrix composites [108-111]. Carbon nanotubes can be conceptualized as cylinders rolled from a graphene sheet and capped at both ends by hemispheres of fullerenes. Theoretical calculation reveals that the electrical properties of carbon nanotubes can range from metallic to semiconducting behavior, depending on the chirality and diameter of the nanotubes. On the other hand, $\mathrm{B}-\mathrm{C}-\mathrm{N}$ nanotubes are insulators with a $\sim 5.0 \mathrm{eV}$ gap independent of these parameters. This implies that the relative B/C/N ratios and/or $\mathrm{BN}$ rich and $\mathrm{C}$-rich domain special arrangements, rather than tube helicity and diameter, are controlling the $\mathrm{B}-\mathrm{C}-\mathrm{N}$ nanotube electrical response [112].

Conventional chemical vapor deposition of hydrocarbons over metal catalyst has been a typical method to produce various forms of carbon fibers, filaments and multi-walled nanotubes [113-116]. The first step in a CVD process involves the absorption and decomposition of hydrocarbon molecules on transition metal ( $\mathrm{Fe}, \mathrm{Ni}, \mathrm{Co}$ ) particles. The carbon atoms diffuse into the interior of the catalyst to form a metal-carbon solid state reaction. Subsequent precipitation of carbon from the supersaturated catalyst particle then occurs and leads to the formation of a carbon tube structure [113-116]. Recently, Wang et al. attempted to use MW-CVD to produce various morphologies of carbon nanotubes by regulating the preparation parameters. However, they offered no explanations for the mechanisms responsible for the formation of various shapes of carbon nanotubes [117]. Fig. 19a-h shows TEM 

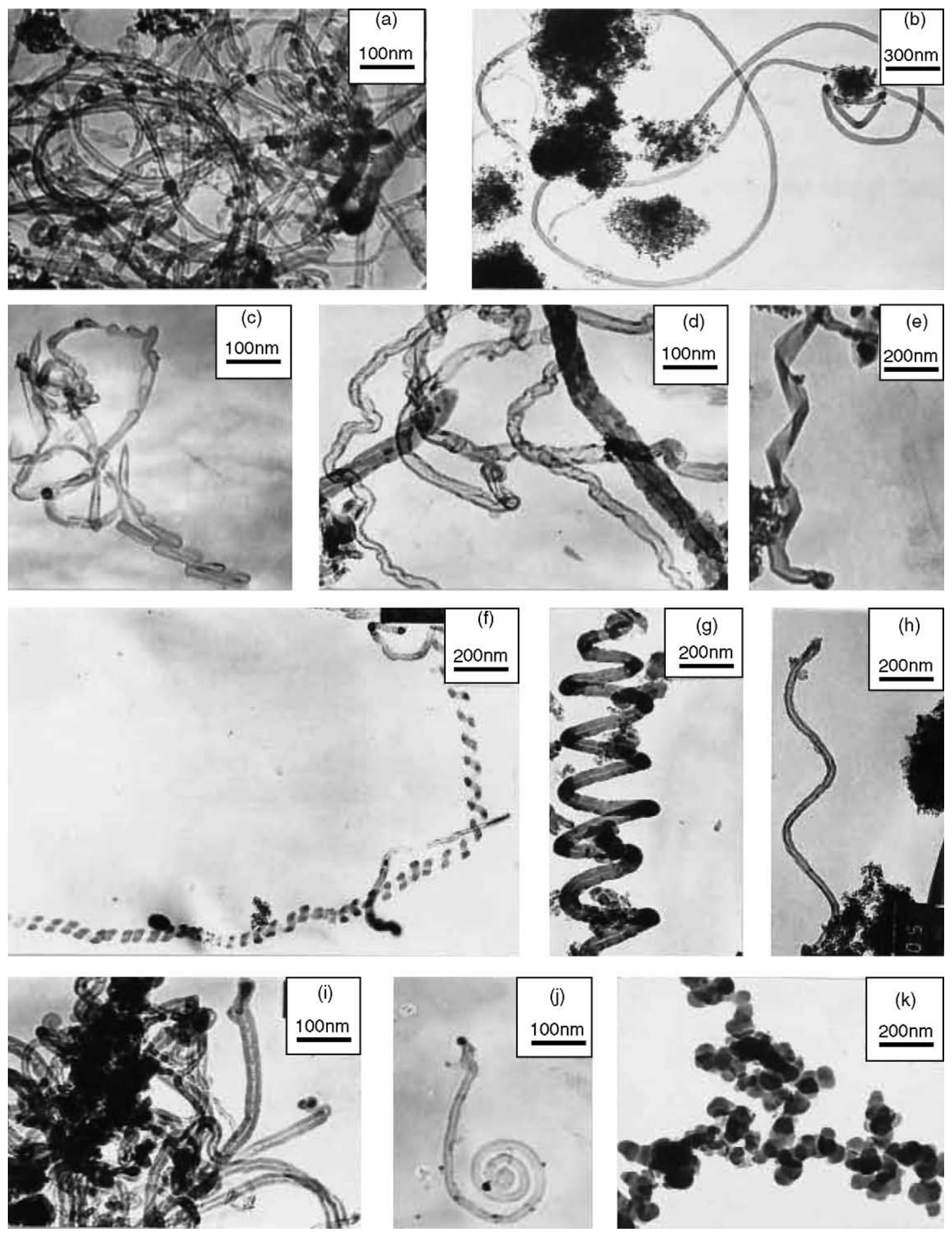

Fig. 19. TEM images of carbon nanostructures prepared by microwave plasma-enhanced CVD (reprinted from [117] with permission from Elsevier). 
micrographs of carbon nanotubes prepared by MW-CVD using a mixture of $\mathrm{CH}_{4}, \mathrm{H}_{2}$ and $\mathrm{Ar}$ reacting gases. Fe, Ni or Co catalysts were used. The multi-walled carbon nanotubes consist of a hollow core and coaxial tubes of multiple graphitic sheets. The outer diameters of nanotubes range from 10 to $30 \mathrm{~nm}$ and the aspect ratio is in the range of 100 to 1000 (Fig. 19a and b). Unlike SiC nanorods which are straight [96], the carbon nanotubes with a circular cross section are smooth and curvy (Fig. 19d). Moreover, ribbon-like (Fig. 19e), spring-shaped nanotubes with different radii and pitches (Fig. 19f and g) as well as necklace-chains (Fig. 19k) are also observed. Some black dots are evident in the spring and necklace-shaped nanotubes. They are considered to be the sites of nuclei. In a similar work, Si nanowires have been found to exhibit several morphologies, e.g. spring, fish-bone and necklace. Tang et al. [118] explained the formation of Si-nanowires of various shapes in terms of a 'two-step' growth model based on the vapor-liquid-solid (VLS) mechanism [119]. In this two-step growth model, the nucleation step is divided into monocentric and polycentric while the growth step into periodic stable and periodic unstable. Thus, the combination of different nucleation and growth processes can give rise to various shapes of Si nanowires. As an example, the formation process of the spring-shaped formation is considered to be due to monocentric nucleation and periodic stable growth, while the necklace-shaped formation is associated with polycentric nucleation and periodic unstable growth. Periodic growth in the spring makes its diameter remain unchanged. However, periodic unstable growth makes the diameter of the nanowire change regularly [118]. The reason for the periodic unstable growth is complicated. It is possibly associated with the self-oscillatory effect in VLS mechanism. The effect is related to many parameters, such as growth temperature, unstable interface of liquid and solid, etc [120]. From this, it appears that the two-step growth model based on the VLS mechanism can explain the formation of various shapes of carbon nanotubes as illustrated in Fig. 19.

The VLS model was originally developed by Wagner and Ellis for the fabrication of micrometersized whiskers in 1960 [119]. The process involves the dissolution of gaseous reactants into nanosized liquid droplets of a metal catalyst, follwed by nucleation and growth of single crystalline rods and wires. This growth is mainly induced by the liquid droplets. The droplet size remains essentially unchanged during the entire process of wire growth. Each liquid droplet serves as a catalyst to assist the subsequent growth and limits the lateral growth of an individual wire. The solubility of forming liquid alloy with the target material is a major requirement of this model. Once the liquid droplet is saturated, the growth can occur at the solid-liquid interface. The VLS model is a well established mechanism responsible for the growth of nanowires and nanotubes of various inorganic materials, e.g semiconductors, oxides. [121-130].

Laser CVD is associated with the deposition of chemical vapors using a laser beam generated from sources like $\mathrm{CO}_{2}, \mathrm{Nd}$ :YAG and excimer. This process offers deposition of films of good quality at a lower temperature with better control of composition and deposition area. For example, silicon nitride film could be deposited at $200{ }^{\circ} \mathrm{C}$ using laser CVD whereas it is deposited at 850 and $450{ }^{\circ} \mathrm{C}$ by thermal activated CVD and plasma enhanced CVD, respectively [131-133]. In the case of nanoparticles, tungsten powders of $54 \mathrm{~nm}$ can be synthesized from $\mathrm{WF}_{6} / \mathrm{H}_{2} / \mathrm{M}(\mathrm{M}=\mathrm{Ar}, \mathrm{Kr}, \mathrm{Ne}, \mathrm{Xe})$ gas mixtures irradiated with an ArF excimer laser [134]. Moreover, ceramic $\mathrm{Si}-\mathrm{C}-\mathrm{N}$ nanometric powders can be obtained from laser-induced gas phase reactions of $\mathrm{SiH}_{4}$, amines and $\mathrm{NH}_{3}[135,136]$. The incorporation of $\mathrm{Si}-\mathrm{C}-\mathrm{N}$ powders with sizes of $25-30 \mathrm{~nm}$ into aluminum improves its tensile and creep properties considerably [137].

\subsubsection{Spray conversion processing}

This route involves the atomization of chemical precursors into aerosol droplets that are dispersed throughout a gas medium. The aerosols are then transported into a heated reactor where the solution is evaporated or combusted to form ultrafine particles or thin films. This technique is versatile and 


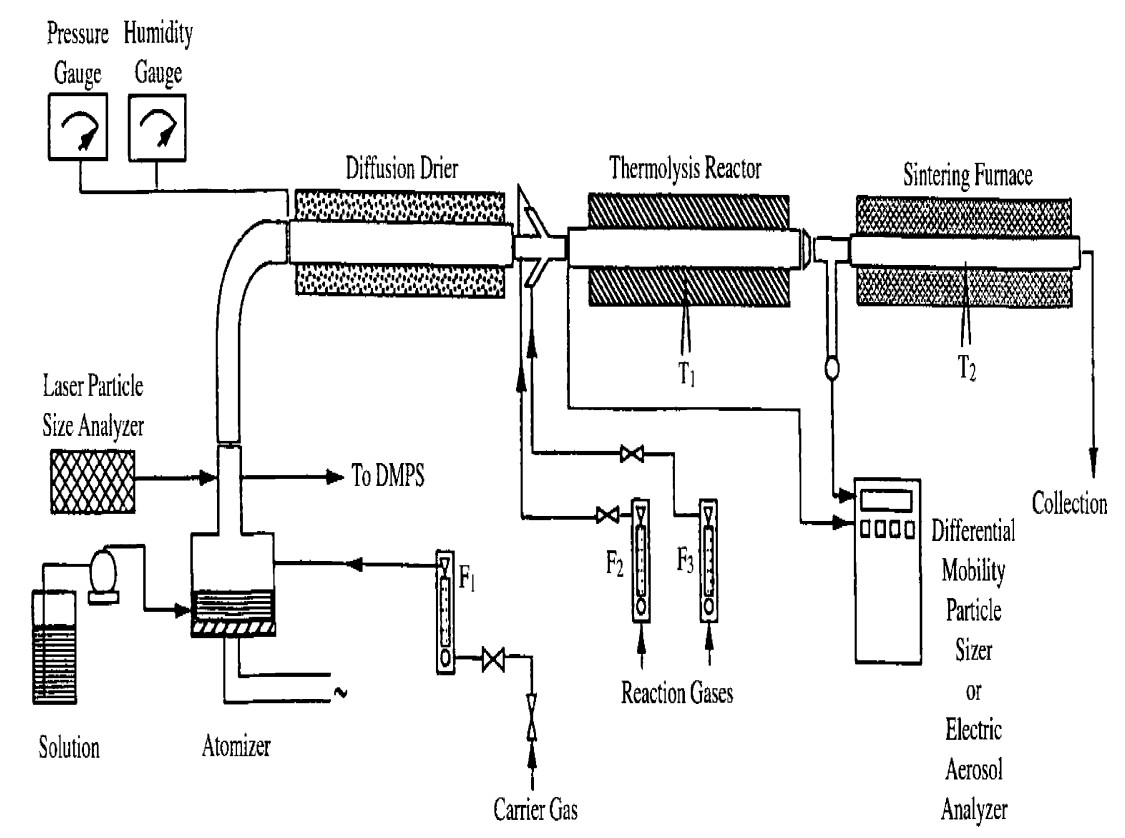

Fig. 20. Schematic representation of spray pyrolysis equipment (reprinted from [138] with permission from The American Ceramic Society).

inexpensive because of the availability of various low cost chemical solutions. Various aerosol generators including pressure, electrostatic, and ultrasonic atomizer, have been used for atomization purposes. These atomizers will affect the droplet size, rate of atomization and droplet velocity. The most commonly used aerosol processing methods is spray pyrolysis as shown in Fig. 20 [138]. In the process, aqueous solution is atomized into a series of reactors where the aerosol droplets undergo evaporation and solute condensation within the droplet, drying, thermolysis follow by sintering. Nanoparticles can be prepared directly, or synthesized from droplets or by liberating the individual crystallites comprising the spray pyrolysis derived particles after the thermolysis stage. Spray pyrolysis can be used to prepare several metal oxide nanoparticles such as $\mathrm{ZnO}, \mathrm{ZrO}_{2}$ and $\mathrm{Al}_{2} \mathrm{O}_{3}$ [138].

Another type of gas condensation technique that uses the liquid chemical precursors as the starting materials is combustion flame spraying. This process offers advantages such as low cost, single step processing, versatility and high production rates [139-142]. In the combustion flame spraying process, burning a fuel/oxygen mixture in spray torch [140] or flat-flame burner [141] generates a flame. Chemical precursors are vaporized in the hot zone of the flame and pyrolysis occurs in the thin hot zone (Fig. 21a). Flame-droplet interactions lead to the formation of nanoparticles. High quality particles can only be produced in a low-pressure chamber where the flat flame burner is housed (Fig. 21b). This precludes contamination and ensures the temperature profile across the entire burner surface is uniform. Low-pressure combustion flame spraying is often referred to as combustion-flame chemical vapor condensation (CF-CVC). The chemical precursors can be liquid, gaseous or solid. Chemical vapor condensation (CVC) in an alternative to the inert-gas phase condensation approach in which the evaporator in the IGC system is replaced with other sources such as burning flame, hot wall furnace and microwave plasma. The major advantage of the plasma-assisted pyrolysis in contrast to thermal activation is the low reaction temperature which reduces the tendency for agglomeration of nanoparticles [142,143]. Fig. 22 shows a typical TEM micrograph of cube-shaped and crystalline $\mathrm{CoO}_{x}$ nanopowders (10-35 nm) prepared by the flat flame burner technique [142]. Combustion flame 

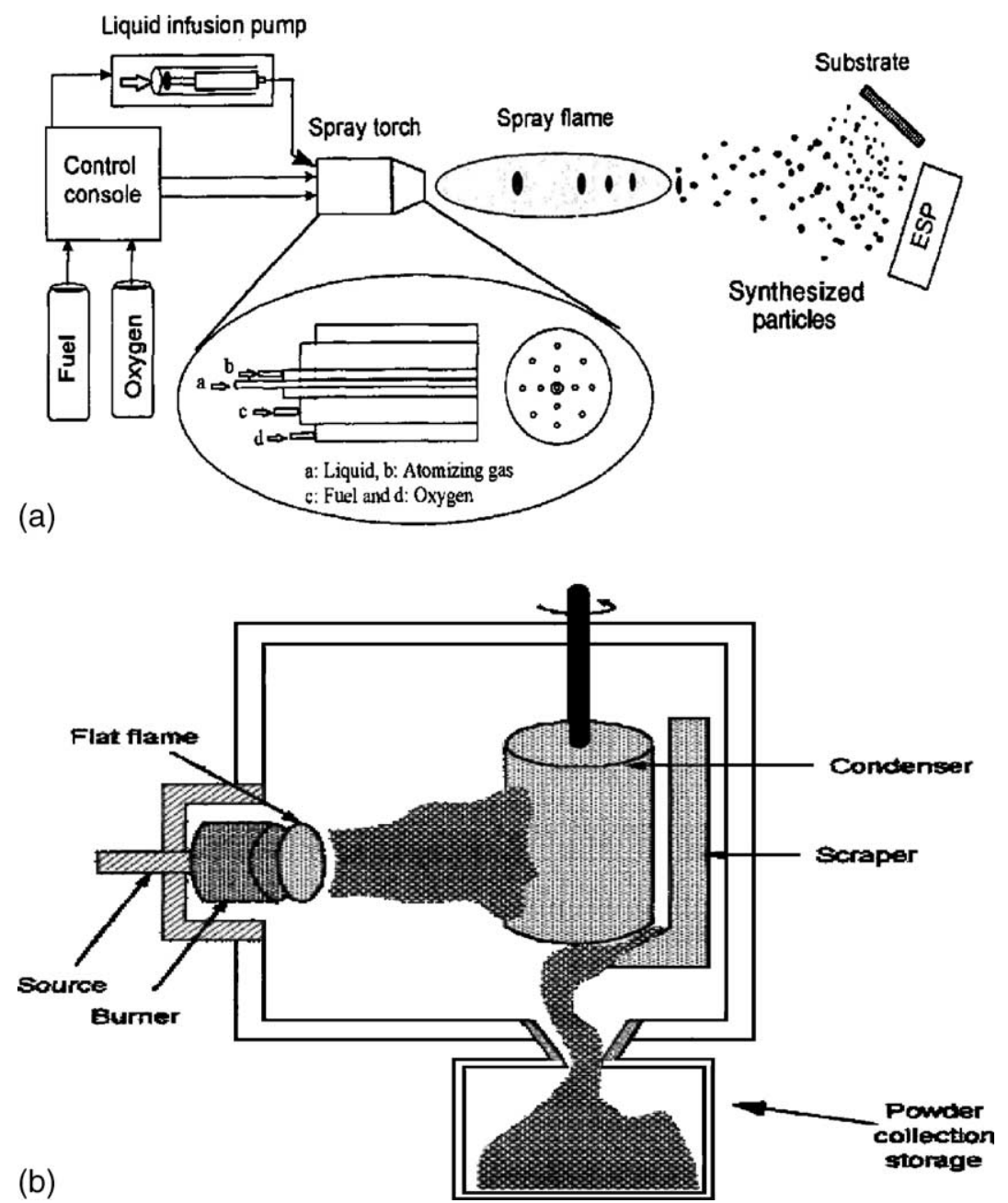

Fig. 21. Schematic diagrams of flame spraying systems equipped with (a) spray torch, and (b) flat flame burner (reprinted from [140,141] with permission from Elsevier).

spraying has been used to produce a variety of high purity and non-agglomerated metal oxide nanoparticles such as $\mathrm{TiO}_{2}, \mathrm{Al}_{2} \mathrm{O}_{3}, \mathrm{ZrO}_{2}, \mathrm{~V}_{2} \mathrm{O}_{5}$ and $\mathrm{Y}_{2} \mathrm{O}_{3}-\mathrm{ZrO}_{2}$ [140,142]. Fig. 23 shows a schematic diagram of hot wall reactor $\mathrm{CVC}$ employing a gas-to-particle conversion process. Hot wall reactor CVC can yield ceramic particles with sizes somewhat slightly smaller than those produced by the combustion flame process. The CVC process has been used to synthesize zirconia powders, with controlled particle size in the range of 2-20 nm [144,145]. Zirconia powders synthesized via flame combustion are reported to exhibit a size range of $12-150 \mathrm{~nm}$ [140].

\subsection{Liquid route}

\subsubsection{Sol-gel process}

The sol-gel processing method has been used for producing metal oxide and ceramic powders with high purity and high homogeneity for many years. The sol-gel route offers a degree of control of composition and structure at the molecular level. The process involves the generation of a colloidal 


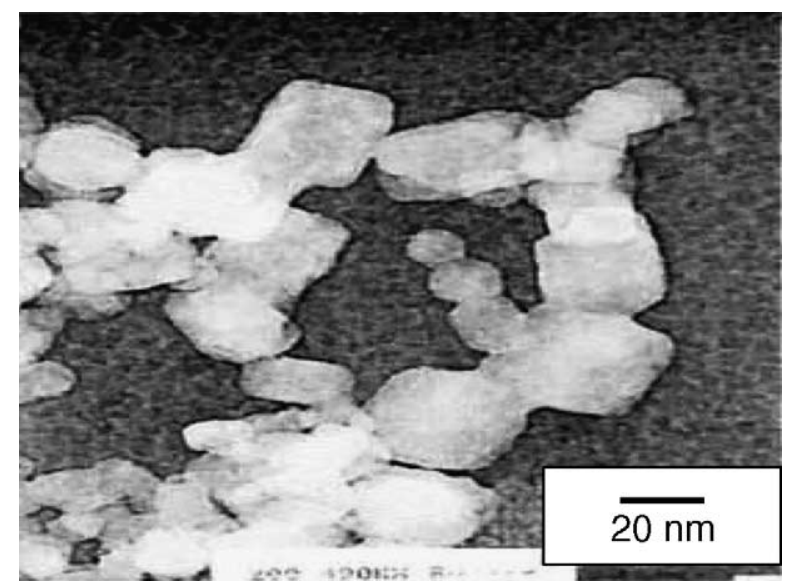

Fig. 22. TEM micrograph of cube-shaped and crystalline $\mathrm{CoO}_{x}$ nanopowders consisting of both $\mathrm{CoO}$ and $\mathrm{Co}_{3} \mathrm{O}_{4}$ phases (reprinted from [141] with permission from Elsevier).

suspension ('sols') which are subsequently converted to viscous gels and solid materials. Ebelman produced the first silica gels in 1846 and Cossa synthesized alumina gels in 1870 [146,147]. Since then, aerogels of zirconia, silazane, borate and other ceramics were synthesized using the sol-gel technique $[148,149]$. In the process, reactive metal precursors were initially hydrolized, followed by condensation and polymerization reactions. Metal alkoxides are metalorganic compounds having an organic ligand attached to a metal or metalloic atom. They are the result of direct or indirect reactions between a metal $\mathrm{M}$ and an alcohol $\mathrm{ROH}$. Typical examples are methoxide $\left(\mathrm{OMe} ; \mathrm{MOCH}_{3}\right)$ and ethoxide $\left(\mathrm{OE}_{\mathrm{t}} ; \mathrm{MOC}_{2} \mathrm{H}_{5}\right)$. During hydrolysis, the alkoxy groups (OR) are replaced by hydroxo ligands

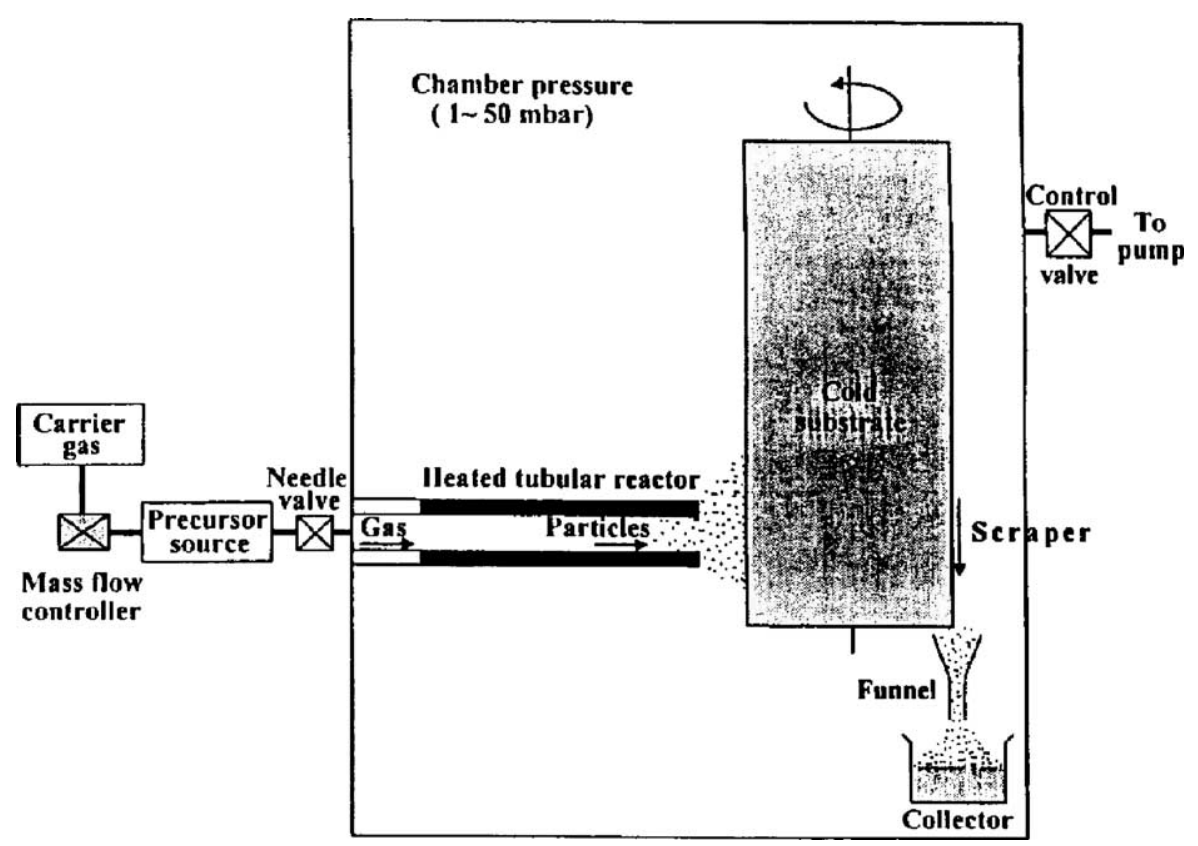

Fig. 23. A schematic of the chemical vapor condensation (CVC) process (reprinted from [145] with permission from Elsevier). 
$(\mathrm{OH})$, i.e.

$$
\mathrm{M}(\mathrm{OR})_{z}+\mathrm{H}_{2} \mathrm{O} \rightarrow \mathrm{M}(\mathrm{OH})(\mathrm{OR})_{z-1}+\mathrm{ROH}
$$

where $\mathrm{R}$ is an alkyl group, $\mathrm{C}_{n} \mathrm{H}_{2 n+1}$. The mechanism of this reaction involves the addition of a negatively charged $\mathrm{HO}^{\delta-}$ group to the positively charged metal center $\left(\mathrm{M}^{\delta+}\right)$ followed by the removal of $\mathrm{ROH}[150,151]$ :

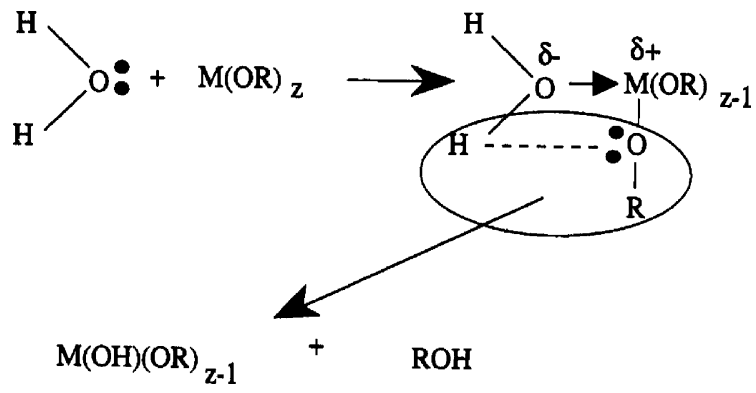

Several factors are known to affect the hydrolysis reaction [150-152]. These include:

(a) The nature of the alkyl group.

(b) The nature of the solvent.

(c) The concentration of each specie in the solvent.

(d) The temperature.

(e) The water to alkoxide molar ratio.

(f) The presence of acid or base catalysts.

Subsequent condensation eliminates either water or alcohol to produce metal oxide or hydroxide linkages. In this process, two mononuclear complexes of $\mathrm{M}$, each comprising only one metal $\mathrm{M}$, can react with one another to form a polynuclear complex consisting of two metal atoms. Condensation occurs only when at least one hydroxo ligand is bonded to the cation $\mathrm{M}$, and is designated as $\mathrm{M}-\mathrm{OH}$ for simplicity. Condensation can proceed via olation and oxolation reactions. Olation is a reaction by which hydroxo or "ol" bridge $\mathrm{M}-\mathrm{OH}-\mathrm{M}$ bond is formed between two cations whilst oxalation involves the formation of oxo bridges $\mathrm{M}-\mathrm{O}-\mathrm{M}$ between to metal cations $\mathrm{M}$ [150]:

$$
\begin{aligned}
& \text { olation: } \quad \mathrm{M}-\mathrm{OH}+\mathrm{M}-\mathrm{OH}_{2} \rightarrow \mathrm{M}-\mathrm{OH}-\mathrm{M}+\mathrm{H}_{2} \mathrm{O} \\
& \text { oxalation : } \mathrm{M}-\mathrm{OH}+\mathrm{H}-\mathrm{OM} \rightarrow \mathrm{M}-\mathrm{O}-\mathrm{M}+\mathrm{H}_{2} \mathrm{O} \\
& \mathrm{M}-\mathrm{OH}+\mathrm{ROM} \rightarrow \mathrm{M}-\mathrm{O}-\mathrm{M}+\mathrm{ROH}
\end{aligned}
$$

The "ol" or "oxo" bridges between two metal atoms lead to the formation of condensed oxide or hydroxide species. Under acid conditions, three-dimensional solid phase networks consisting of extended linear M-O-M chain polymers are developed [153]. Inorganic polymerization is believed to occur in three stages during acid-catalyzed condensation [154]:

(i) Polymerization of monomer units to form particles.

(ii) Growth of particles.

(iii) Linking of particles into chains, then solid networks that extend throughout the liquid medium, thickening it to a gel. 
In acid solution, the sol-to-gel transition allows the solid phase to be shaped into films, fibers and monoliths. For preparing coating films and fibers, the sol must exhibit spinnability. It appears that only solutions containing long-chained polymers are spinnable [155]. Films are generally coated on the surface of the substrate via spin-coating and dipping processes. Gel fibers are made by fiberdrawing from the viscous alkoxide solution at or near room temperature. The flow chart of sol-gel processing is schematically shown in Fig. 24 [151]. In contrast, a colloidal sol is generated upon basic hydrolysis of metal alkoxides $[150,156]$. The gel is colloidal when the solid network is made of round sol particles.

Removal of the solvents and appropriate drying are important steps to achieve gel densification. When a solvent is evaporated from the gel under atmospheric conditions, capillary pressure due to the interfacial tension of the solvent develops a high stress on the gel network. This leads to considerable shrinkage and fracture of the gel during drying. The resultant hard, glassy and porous product is called a xerogel. In the case where the liquid within the gel is removed above its critical temperature and pressure (hypercritical) in an autoclave, the capillary pressure can be eliminated. The product thus obtained is referred to as an aerogel $[157,158]$. Aerogel is generally armorphous and exhibits several

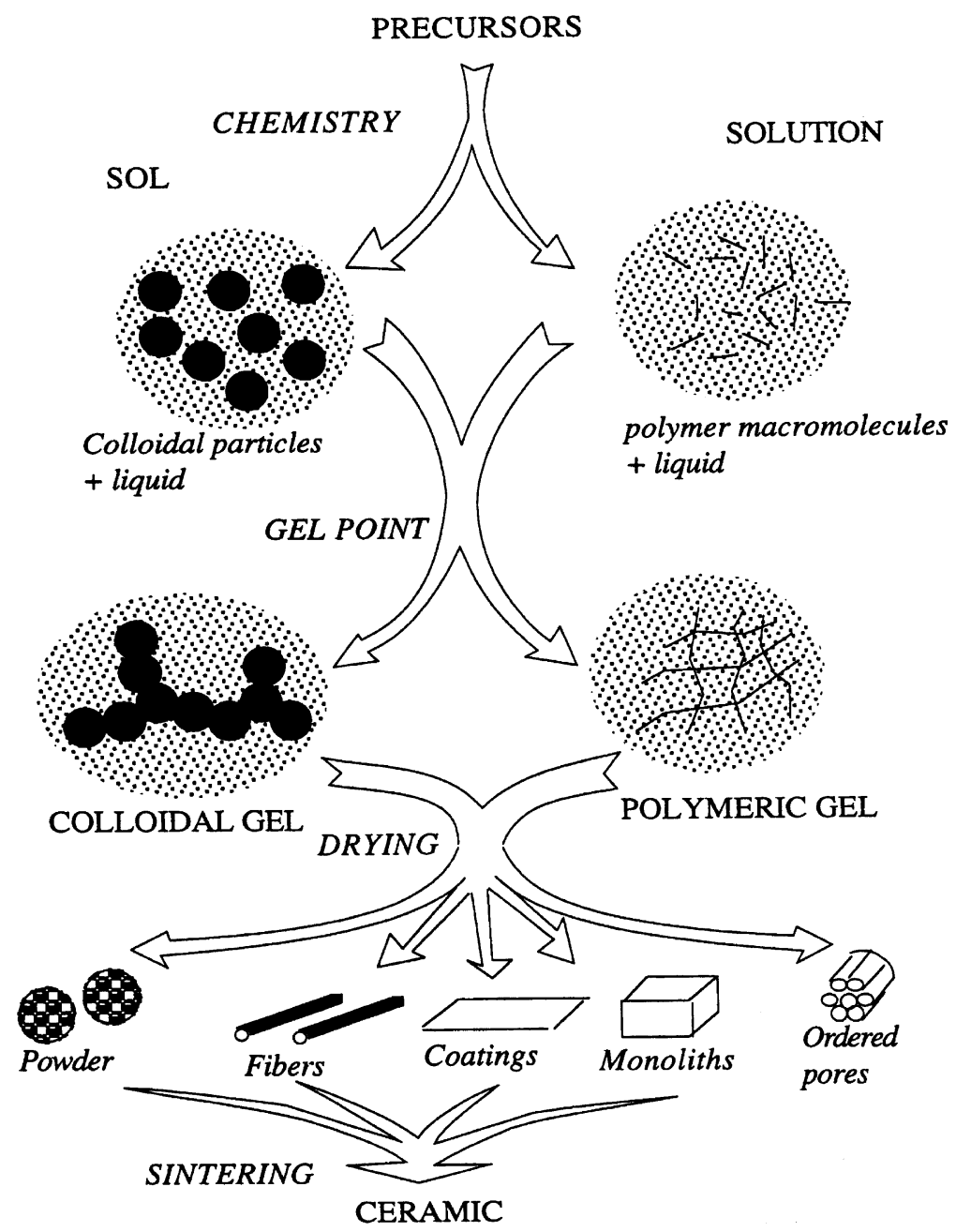

Fig. 24. Flow chart of so-gel processes (reprinted from [151] with permission from Kluwer Academic Publishers). 
unique properties such as high surface areas and high porosities, low densities, and low conductivities [159].

The most common studied metal alkoxides is silicon tetraethyl orthosilicate (TEOS; $\left.\mathrm{Si}\left(\mathrm{OC}_{2} \mathrm{H}_{5}\right)_{4}\right)$. The TEOS precursor can react readily with water via the following reaction:

$$
\mathrm{Si}(\mathrm{OR})_{4}+\mathrm{H}_{2} \mathrm{O} \rightarrow \mathrm{Si}(\mathrm{OR})_{3}(\mathrm{OH})+\mathrm{ROH}
$$

As the hydrolysis of silicon alkoxides is very slow, the conversion of metal precursor molecules into trialkoxy silanol $\mathrm{Si}(\mathrm{OR})_{3}(\mathrm{OH})$ proceeds more rapidly by adding acid or base catalysts. Depending on the amount of water and catalyst present, hydrolysis may proceed partially in which the metal alkoxides convert into $\mathrm{Si}(\mathrm{OR})_{4-n}(\mathrm{OH})_{n}$, or may go to completion in which all OR groups of alkoxides are replaced by $\mathrm{OH}[150]$ :

$$
\mathrm{Si}(\mathrm{OR})_{4}+4 \mathrm{H}_{2} \mathrm{O} \rightarrow \mathrm{Si}(\mathrm{OH})_{4}+4 \mathrm{ROH}
$$

Subsequent condensation reaction sequences produce cluster species with $\mathrm{Si}-\mathrm{O}-\mathrm{Si}$ (siloxane) bonds and water or alcohol as the by-products:

$$
\begin{gathered}
(\mathrm{OR})_{3} \mathrm{Si}-\mathrm{OH}+\mathrm{HO}-\mathrm{Si}(\mathrm{OR})_{3} \rightarrow(\mathrm{OR})_{3} \mathrm{Si}-\mathrm{O}-\mathrm{Si}(\mathrm{OR})_{3}+\mathrm{H}_{2} \mathrm{O} \\
(\mathrm{OR})_{3} \mathrm{Si}-\mathrm{OR}+\mathrm{HO}-\mathrm{Si}(\mathrm{OR})_{3} \rightarrow(\mathrm{OR})_{3} \mathrm{Si}-\mathrm{O}-\mathrm{Si}(\mathrm{OR})_{3}+\mathrm{ROH}
\end{gathered}
$$

In silica aerogels, particles of $1-100 \mathrm{~nm}$ are arranged in a highly crosslinked silica solid network [160]. In a recent study, Vasconcelos et al. synthesized colloidal silica particles from hydrolized TEOS, ethanol $\left(\mathrm{C}_{2} \mathrm{H}_{5} \mathrm{OH}\right)$ and deionized water using ammonium hydroxide $\left(\mathrm{NH}_{4} \mathrm{OH}\right)$ as a catalyst [161]. The diameter of spherical silica particles ranges from 0.35 to $1.06 \mu \mathrm{m}$ as a result of varying the molar ratios of TEOS: $\mathrm{NH}_{4} \mathrm{OH}: \mathrm{H}_{2} \mathrm{O}: \mathrm{C}_{2} \mathrm{H}_{5} \mathrm{OH}$ (Table 3). The diameter of the silica particles is increased by raising the amounts of $\mathrm{NH}_{4} \mathrm{OH}$ and $\mathrm{H}_{2} \mathrm{O}$, and by decreasing the amount of $\mathrm{C}_{2} \mathrm{H}_{5} \mathrm{OH}$. Fig. 25a-d are representative SEM micrographs showing the morphologies of the colloidal silica particles obtained from the solutions A, C, E and F, respectively. Moreover, agglomeration of silica particles is observed due the lower solvent concentration $\left(\mathrm{H}_{2} \mathrm{O}\right.$ and $\left.\mathrm{C}_{2} \mathrm{H}_{5} \mathrm{OH}\right)$ as shown in Fig. $25 \mathrm{~b}$. According to the literature, silica nanoparticles can also be prepared simply via thermal decomposition, e.g. oxidation of TEOS or rice husk in a reactor [162,163], and CF-CVC route as mentioned previously [164]. Thermal decomposition of rice husk appears to be more cost effective due to global availability of cheap rice husk [163]. Other process involves the precipitation of silica particles from the silicate solution and buffer with sulfuric acid [165]. The silica naparticles prepared by other methods are listed in Table 4 for the purposes of comparison.

\begin{tabular}{|c|c|c|c|c|c|c|}
\hline Solution & TEOS & $\mathrm{NH}_{4} \mathrm{OH}$ & $\mathrm{H}_{2} \mathrm{O}$ & $\mathrm{C}_{2} \mathrm{H}_{5} \mathrm{OH}$ & $\mathrm{d}(\mu \mathrm{m})$ & $\delta(\mathrm{nm})$ \\
\hline A & 1 & 4 & 16 & 6 & 1.06 & 0.08 \\
\hline B & 1 & 4 & 16 & 24 & 0.65 & 0.05 \\
\hline $\mathrm{C}$ & 1 & 1 & 4 & 6 & 0.43 & 0.13 \\
\hline D & 1 & 10 & 4 & 6 & 0.66 & 0.10 \\
\hline $\mathrm{E}$ & 1 & 10 & 4 & 60 & 0.35 & 0.08 \\
\hline $\mathrm{F}$ & 1 & 10 & 40 & 60 & 0.46 & 0.04 \\
\hline
\end{tabular}

Table 3

Molar ratios of sol-gel silica, particle diameters (d) and standard deviations $(\delta)$ (reprinted from [161] with permission from Elsevier) 


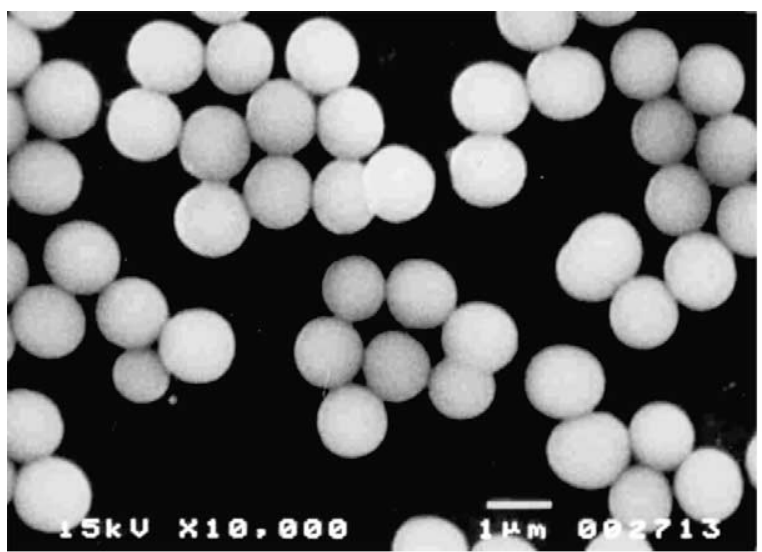

(a)

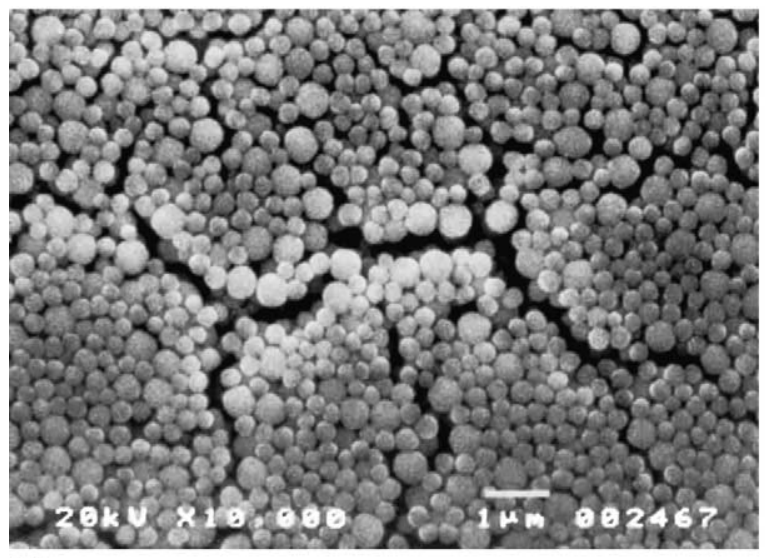

(c)

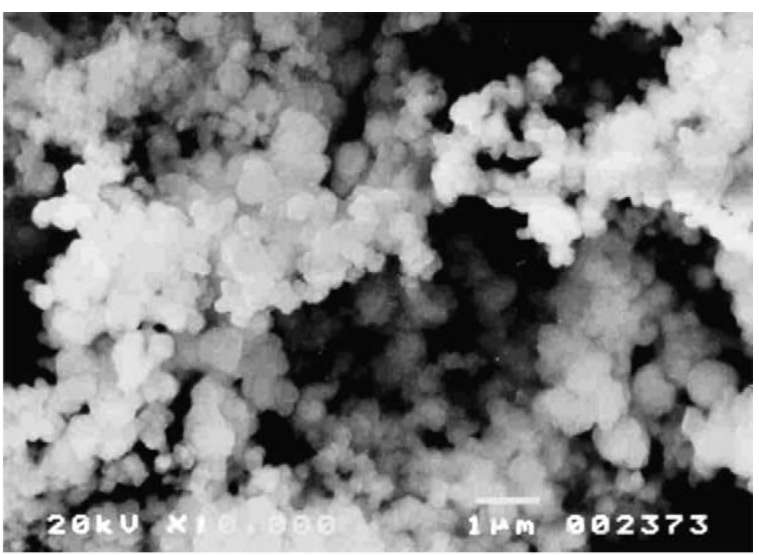

(b)

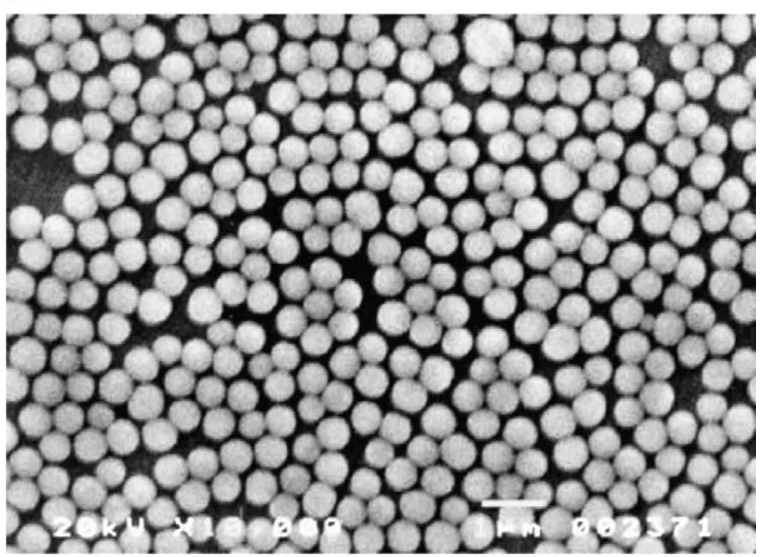

(d)

Fig. 25. Silica particles obtained from (a) solution A, (b) solution C, (c) solution E and (d) solution F (reprinted from [161] with permission from Elsevier).

Alkoxides of transition metals (e.g. titanium, zirconium) are highly reactive towards water. This implies that the rate of hydrolysis and condensation is very high, thus it is difficult to form a stable sol of titania $\left(\mathrm{TiO}_{2}\right)$. Naturally, titania exists in three main crystallographic structures, i.e. rutile, anatase and brookite. Rutile is the only stable phase, whereas anatase and brrokite are both metastable at all temperatures. Many acid catalysts such as nitric acid, hydrochloric acid and acetic acid have been added to lower the reaction rates [166-168]. Apart from these acids, acetylacetone $\left(\mathrm{C}_{5} \mathrm{H}_{8} \mathrm{O}_{2}\right)$ can also moderate the reaction rate. Acetylactone is added as a chelating agent to decrease the reactivity of titanium alkoxides and to form stable colloidal sols of $\sim 5 \mathrm{~nm}$ [169-171]. Under more controllable hydrolysis and condensation reactions, smaller particle sizes with more unique properties can be

Table 4

Properties of silica nanoparticles prepared by other methods

\begin{tabular}{lcll}
\hline Processing method & Diameter $(\mathrm{nm})$ & Purity $(\%)$ & Yield \\
\hline Thermal decomposition of TEOS [162] & $10-40$ & - & Several tens of grams per hour \\
Decomposition of rice husk [163] & 60 & $>99.7$ & - \\
Combustion-flame CVC [164] & $3-50$ & $>99.9$ & $30-50 \mathrm{~g} / \mathrm{h}$ \\
Wet chemical synthesis [165] & $15-30$ & - & - \\
\hline
\end{tabular}


achieved. For example, Masson et al. reported that $\mathrm{TiO}_{2}$ aerogel nanocrystals can be obtained by supercritical evacuation of solvent from gels prepared through $\mathrm{HCl}$ controlled hydrolysis condensation reactions of tinanium isopropoxide in isopropanal [172]. Ding and Liau indicated that the $\mathrm{HCl}$ catalyst favors the synthesis of titania powders with smaller grain size compared to acetylacetone catalyst under the same heat treatment conditions. Further, anatase crystallites are more stable if $\mathrm{HCl}$ is used as catalyst, while pure rutile phase can be more easily obtained if acetylacetone is used as catalyst [173]. According to the literature, various metal oxide nanocrystalline powders, e.g. $\mathrm{SnO}_{2}, \mathrm{Ba}_{2} \mathrm{Ti}_{2} \mathrm{O}_{5}$, $\mathrm{PbTiO}_{3},(\mathrm{~Pb}, \mathrm{La})(\mathrm{Zr}, \mathrm{Sn}, \mathrm{Ti}) \mathrm{O}_{3}$ [174-178], nanocomposite powders containing elemental particulates, e.g. nano- $\mathrm{Ni} / \mathrm{SiO}_{2}, \mathrm{Fe}-\mathrm{Al}_{2} \mathrm{O}_{3}[179,180]$ and oxide nanocomposites, e.g. $\mathrm{Fe}_{2} \mathrm{O}_{3}-\mathrm{SiO}_{2}, \mathrm{NiO}-\mathrm{SiO}_{2}$, $3 \mathrm{Al}_{2} \mathrm{O}_{3}-2 \mathrm{SiO}_{2}[181,182]$ have been synthesized using the sol-gel process.

From these, sol-gel process has been useful for synthesizing only metal oxides as a result of the presence of metal-oxygen bonds in the alkoxide precursor, and the resulting gels are essentially metal hydroxides or oxides. This process has distinct advantages over other tehniques for preparing metal oxide nanoparticles. These include the formation of high purity powders as a result of homogeneous mixing of the raw materials on the molecular level, and the large scale induatrial production of nanopowders. The disadvantage of the process is the high cost of the alkoxide precursors. In some cases, sol-gel route can also be used to prepare nonoxide ceramic powders such as $\beta-\mathrm{SiC}$ [183] and ferroelectrics like $(\mathrm{Pb}, \mathrm{La})(\mathrm{Zr}, \mathrm{Sn}, \mathrm{Ti}) \mathrm{O}_{3}$ (PLZST) [178]. In the former case, heat treatment of the gel product at $1500{ }^{\circ} \mathrm{C}$ in argon atmosphere resulted in $0.6 \mu \mathrm{m}$ spherical agglomerates consisting primariry paricles of $40 \mathrm{~nm}$ in diameter [183]. Recently, $\beta$-SiC nanopowders of 13-30 nm can be synthesized by the chemical vapor reaction of the $\mathrm{SiH}_{4}-\mathrm{C}_{2} \mathrm{H}_{4}-\mathrm{H}_{2}$ system in the temperature range between 1423 and $1673 \mathrm{~K}$. The products are free from agglomeration [184]. $\beta$-SiC powders can be available at relatively low cost by the process of pyrolizing rice husks at $1200-1500{ }^{\circ} \mathrm{C}$. The disadvantage is the larger size of $\beta-\mathrm{SiC}$ powders produced from this process, i.e. within micrometer range [185]. In is worthnoting that transition metal nanorods by reacting carbon nanotubes with volatile metal oxide or metal halide. Thus, $\beta$-SiC nanorods can be produced from reactions of carbon nanotubes with $\mathrm{SiO}$ or $\mathrm{Si}-\mathrm{I}$ reactants. The preferred growth direaction of these nanorods is [ $\left[\begin{array}{lll}1 & 1 & 1\end{array}\right]$,

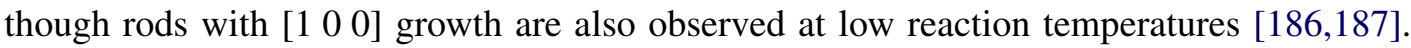

The antiferoelectric ceramics are promising candidates for new ceramic actuators because of their unique characteristics of big and isotropic strain changes under applied electric field, as well as shape memory effects. Nanozized ceramics prepared by the sol-gel method are usually more homogeneous and reactive than those fabricated by conventional solid-state reactions since the mixing of the reagents occurs on a finer scale. The lower processing temperature is also helpful in minimizing the $\mathrm{PbO}$ loss. In lead-based complex perovskites, $\mathrm{PbO}$ evaporates easily at high temperatures resulting in undesirable phases. Sol-gel derived powders of single perovskite phase can be used to produce bulk ceramic samples after sintering at various temperatures [178].

\subsubsection{Wet chemical synthesis}

Solution-based processing routes used for the synthesis of nanoparticles include precipitation of solids from a supersaturated solution [188,189], homogeneous liquid phase chemical reduction [190192] and ultrasonic decomposition of chemical precursors [193-195]. These processes are attractive due to their simplicity, versatility and availability of low cost precursors. Inorganic salt compounds used in the wet chemical synthesis routes are more versatile and economical than alkoxides employed in the sol-gel process. A typical example is the formation of nanocrytalline titania powders via hydrolysis of $\mathrm{TiCl}_{4}$ at lower temperatures [188,189]:

$$
\mathrm{TiCl}_{4}+2 \mathrm{H}_{2} \mathrm{O} \rightarrow \mathrm{TiO}_{2}+4 \mathrm{HCl}
$$


Once the solution becomes saturated, crystallization of titania takes place either through homogeneous or heterogeneous nucleation $[188,189]$. In the latter case, crystal seeds are added to the solutions to promote the crystallization of titania nanoparticles.

Salt reduction is one of the most commonly adopted methods to generate the metal colloid particles. The process involves the dissolution of metal salts in aqueous or non-aqueous environments followed by the reduction of metal cations to the zero-valent state. The nature of the metal salts determines the kind of reducing agent to be applied. To produce transition metal nanoparticles, group 6 metal chlorides such as $\mathrm{CrCl}_{3}, \mathrm{MoCl}_{3}$ and $\mathrm{WCl}_{4}$ are reduced with $\mathrm{NaBEt}_{3}$ in toluene solution at room temperature to form metal colloids of high yield [190]. A typical reaction for forming metal powders is given as:

$$
\mathrm{MCl}_{x}+x \mathrm{NaBEt}_{3} \rightarrow \mathrm{M}+x \mathrm{NaCl}+x \mathrm{BEt}_{3}+\left(\frac{1}{2} x\right) \mathrm{H}_{2}
$$

where $x=3$ for $\mathrm{M}=\mathrm{Cr}$ and $\mathrm{Mo} ; x=4$ for $\mathrm{M}=\mathrm{W}$. However, the reduction of the chromium, molybdenum and tungsten halides with either $\mathrm{LiBEt}_{3} \mathrm{H}$ or $\mathrm{NaBEt}_{3}$ in tetrahydrofuran (THF) solution generates the corresponding metal carbides $\left(\mathrm{M}_{2} \mathrm{C}\right)$ rather than the metals. In another experiment, Lima Jr. et al. [191] have synthesized nanostructured $\mathrm{Fe}_{50} \mathrm{Ni}_{50}$ alloy by ultra rapid autocatalytic chemical reduction of the corresponding transition metal ions in alkaline aqueous solution with hydrazine hydrate $\left(\mathrm{N}_{2} \mathrm{H}_{4} \cdot \mathrm{H}_{2} \mathrm{O}\right)$ at $353 \mathrm{~K}$. The alloy powder is composed of spherical particles with $96 \mathrm{~nm}$ mean size diameter.

Metal nanoparticles can also be generated via ultrasonic and thermal decomposition of metal salts or chemical precursors. Recently, ultrasonic waves have been employed to stimulate chemical reaction of inorganic salts. Sonication of argon-saturated aqueous solutions of $\mathrm{NaAuCl}$ and $\mathrm{PdCl}_{2}$ results in the formation of noble metal AuPd alloy nanoparticles [193]. Power ultrasonic waves can stimulate certain novel chemical processes due to the formation of localized hot spots in the liquid of extremely high temperatures $(\sim 3000 \mathrm{~K})$ and high pressures $(\sim 1000 \mathrm{~atm})$. The main event in the process is the nucleation, growth and collapse of cavitation bubbles formed in the liquid. The cooling achieved during the cavitation collapse is estimated to be greater than $2 \times 10^{9} \mathrm{~K}$. This process is commonly referred to as sonochemical method. Transition metal nanoparticles can be produced via sonication of their respective chemical precursors [194-197]. For example, Suslick et al. sonicated $\mathrm{Ni}(\mathrm{CO})_{4}$ under argon atmosphere to obtain amorphous nickel [194]. Gonsalves et al. prepared nanostructured $\alpha-\mathrm{Fe}$ either either via sonication or thermal decomposition of $\mathrm{Fe}(\mathrm{CO})_{5}$ solution [195]. One disadvantage of the sonication process is the difficulty in controlling the resulting particle size and distribution due to the agglomeration of particles into a porous coral-like microstructure [195].

\subsection{Solid route}

\subsubsection{Mechanical alloying/milling}

The mechanical alloying/milling process was originally developed by Benjamin of the International Nickel Company for the production of oxide dispersion strengthened (ODS) superalloys [198]. During the process, raw powder particles with a size of several microns experience severe plastic deformation, i.e. undergo a repetitive cold welding and fracturing mechanism [199]. Vibratory mill, planetary mill, uniball mill and attritor mill are commonly used to perform mechanical alloying/ milling. Among these, the attritor mill has the highest capacity of powder charge [200,201]. Mechanical alloying (MA) is now recognized as a versatile process for the fabrication of a broad range of nanocrystalline powders. These powders include ODS alloys [198], amorphous alloys [202,203], nanocrystalline metals/alloys [204-213] and supersaturated solid solution [214,215]. The disadvantage of ball-milling for making nanocrystalline powders is the contamination of products from the milling media (balls and vial) and atmosphere. For example, the powders may be 
contaminated with Fe if steel balls and containers are used. The Fe impurity level from the high energy shaker mill is much larger than that from the conventional mill [214].

Eckert et al. studied the mechanical alloying process of fcc metals (e.g. $\mathrm{Al}, \mathrm{Cu}, \mathrm{Ni}, \mathrm{Pd}, \mathrm{Rh}$ and Ir) using a high energy shaker mill [206]. The minimum grain size obtained was observed to vary with melting temperature or the bulk modulus. In other words, the higher the melting point and the bulk modulus are, the smaller the final grain size of the powder is. Further, the grain size decreases and the lattice strain increases with milling time. In the case of low melting point elements (e.g. Al), partial recovery takes place via thermal processes during milling. These materials are heavily coldworked; much of the strain energy expended in the milling is stored. When the materials are thermally acticated in the recovery temperature range, internal stresses are relieved. Thus, the ultimate grain size achievable by MA is determined by the competition between the heavy mechanical deformation introduced during milling and the recovery behavior of the metal [206]. Oleszak and Shingu [208] then studied the influence of low energy ball milling (i.e. attrition milling) on the crystallite size, lattice strain and storage of deformation energies of bcc metals (e.g. $\mathrm{Fe}, \mathrm{W}$ ) and fcc metals (Al, Ag, $\mathrm{Cu}$ and $\mathrm{Ni}$ ). They reported that low energy ball milling of fcc and bcc metals leads to a refinement of the crystallite size to the nanometer scale. The final grain sizes vary between 5 and $25 \mathrm{~nm}$, scaling inversely with the melting points of the respective elements. This is in good agreement with the results of Eckert et al. [206] for nanocrystalline metals prepared by high energy ball milling.

Koch analyzed the results of several workers, and plotted the variation of minimum grain size of bcc, fcc and hcp metals with melting temperature as shown in Fig. 26 [216]. Apparently, fcc metals with lower melting points (e.g. $\mathrm{Al}, \mathrm{Ag}, \mathrm{Cu}$ and $\mathrm{Ni}$ ) show a clear inverse dependence of minimum grain size on melting temperature. On the contrary, the minimum grain size for the bcc and hcp metals, and for the fcc metals with the higher melting temperature ( $\geq T_{\mathrm{m}}$ for $\mathrm{Pd}$ ), exhibits essentially constant value with melting temperature. For these elements, it appears that the minimum grain size is in the order: $\mathrm{fcc}<\mathrm{bcc}<\mathrm{hcp}$. Though these workers employed various mills of different milling energies, Koch indicated that the total strain rather the milling energy and contamination is responsible for determining the nanocrysalline grain size. Fig. 27 shows the variation of lattice strain determined from XRD line broadening with the reciprocal grain size [216]. With the exception of Pd and Ti, the

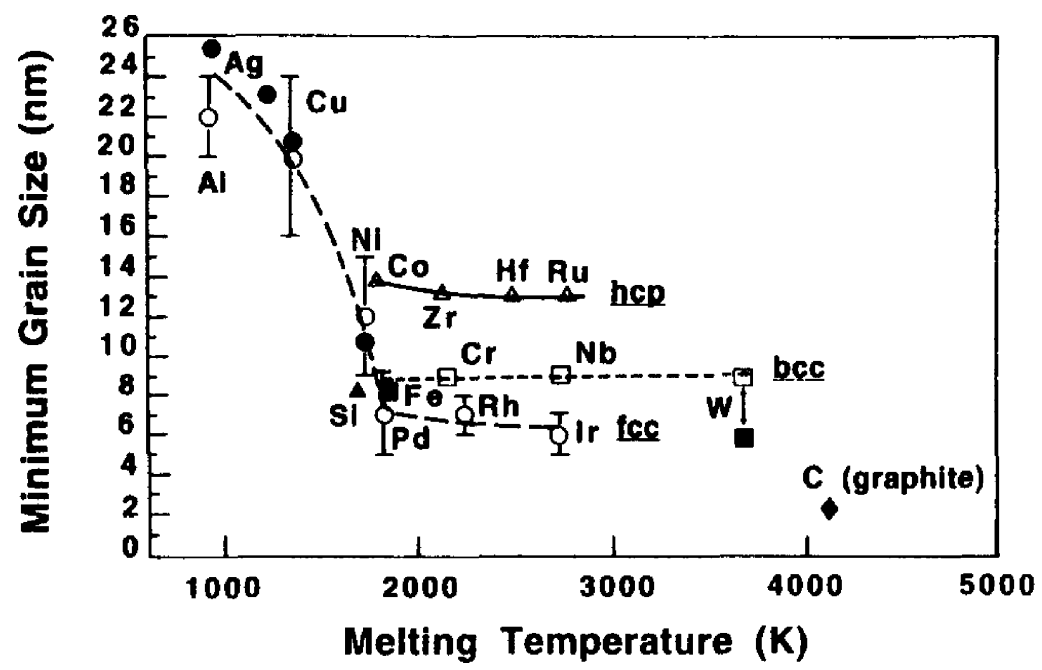

Fig. 26. Variation of minimum grain size with melting temperature for bcc, fcc and hcp metals (reprinted from [216] with permission from Elsevier). 


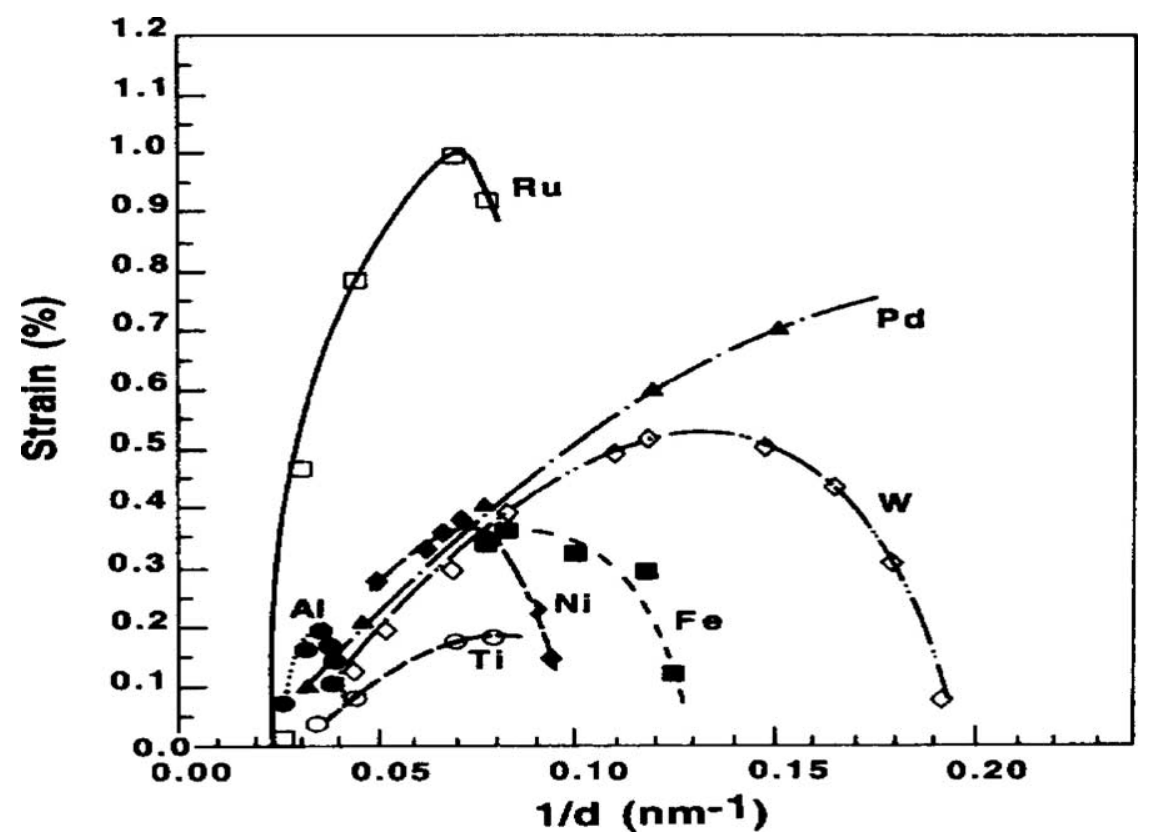

Fig. 27. Lattice strain vs. reciprocal grain size for bcc, fcc and hcp metals (reprinted from [216] with permission from Elsevier).

lattice strain of elements exhibits a maximum in the plot of strain versus $1 / d$. A maximum at a certain strain level has been suggested to result from a change in the deformation from plastic deformation via generation and movement of dislocations to grain boundary sliding during MA processing [204]. However, grain boundary sliding would not refine further the microstructure as the grain size continues to decrease after the strain maximum is reached.

It is noted that the ball-milled metal and alloy powders contain a very large stored deformation enthalpy compared to conventional cold working methods. Large deformation enthalpy of up to $40 \%$ of the enthalpy of fusion is stored in the material. The stored enthalpy can be determined by integrating the DSC exothermic peak [206,208]. The higher stored energy in nanocrytalline metals has been attributed to the high dislocation density [211] and grain boundary strains [208]. Upon annealing at elevated temperatures, the stored energy can normally revert to a lower energy state by structural evolution during recovery and recrystallization [217].

The grain growth in nanocrystalline Fe produced by high energy ball milling [40] was mentioned in previous section. It is discussed in more detail here. The milled Fe powders, having an average grain size of $9.4 \mathrm{~nm}$, were annealed in vacuum at a temperature range of $625-875 \mathrm{~K}$ for various periods of time. Fig. 28 shows the grain size vs. annealing time for MA Fe. It is apparent that the grain size increases rapidly at short annealing times and then continues to grow at a very low rate. For the lower three annealing temperatures $(625,675$ and $725 \mathrm{~K})$, the grain size levels off at $\sim 20 \mathrm{~nm}$. At higher annealing temperatures $(805,825$ and $875 \mathrm{~K})$, the grain size stabilizes at $\sim 60 \mathrm{~nm}$. The grain size data at two annealing temperature regimes were fitted using Eqs. (E1) and (E4), respectively. For the low annealing temperature regime, an activation energy for grain growth determined from Eq. (E1) is $125 \mathrm{~kJ} / \mathrm{mol}$, which is lower than both the activation energies for grain boundary $(174 \mathrm{~kJ} / \mathrm{mol})$ and self diffusion (251-282 kJ/mol) in Fe. For the higher annealing temperature regime, Eq. (E4) yields an activation energy of $248 \mathrm{~kJ} / \mathrm{mol}$, which is close to the values found for grain growth in polycrystalline 


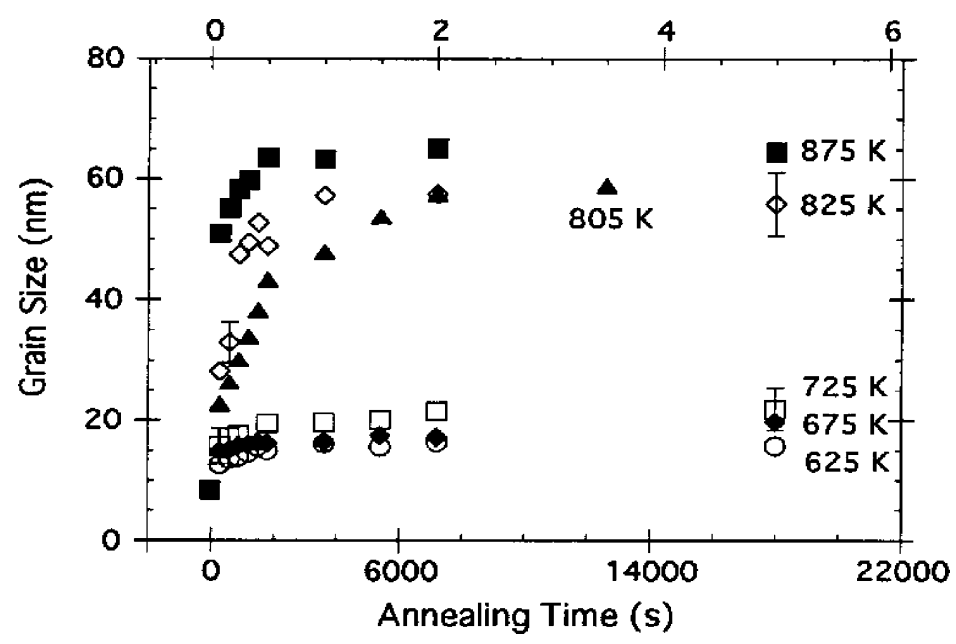

Fig. 28. Grain size as measured by XRD as a function of annealing time for nanocrystalline Fe annealed at various temperatures between 625 and $875 \mathrm{~K}$ (reprinted from [40] with permission from Elsevier).

$\mathrm{Fe}$ and that for self diffusion in Fe. The two values for the activation energy imply that two different mechanisms governing the grain growth during annealing of MA Fe [40].

In a recent study, Zhou et al. employed DSC, XRD and TEM to investigate the recovery and recrystallization of cryomilled $\mathrm{Al}-7.6 \mathrm{Mg}$ alloy subjected to annealing in argon environment [218]. They reported that the microstructure of the as-milled $\mathrm{Al}-7.6 \mathrm{Mg}$ alloy is heterogeneous and consists of high-density dislocations, twins and non-equilibrium grain boundaries. On heating the as-milled powders, two stages of enthalpy released occurred, i.e. recovery in the first stage from 100 to $230{ }^{\circ} \mathrm{C}$, and recrystallization in the second stage at higher temperatures up to $370{ }^{\circ} \mathrm{C}$. Grain coalescence is highly feasible during the grain coarsening in recovery stage, yielding large and elongated grains [218].

More recently, Zhang et al investigated the evolution of microstructure of hcp Zn metal prepared by ball milling [212,213]. The typical TEM micrographs of $\mathrm{Zn}$ metal ball milled for different times at room temperature are shown in Fig. 29a-c. Obviously, the grain size of $\mathrm{Zn}$ decreases with milling time as expected, and reaches a value of $\sim 23 \mathrm{~nm}$ after milling for $25 \mathrm{~h}$. Ball milling at liquid nitrogen temperature (cryomilling) can produce much smaller average grain size in a shorter milling time (Fig. 30). It is believed that dynamic recovery and cold welding are reduced significantly during cryomilling, thus the grain size is refined much faster to a minimum value [213]. In this case, liquid nitrogen provides control of the processing temperature and reduces contamination of the powders during milling. Liquid nitrogen also serves as a reactive agent to form nitrides and oxide particles that facilitate in retarding grain growth of the synthesized powders of $\mathrm{Al}$ and its alloys during MA processing [200,201]. However, cryomilling of pure Fe does not result in enhanced thermal stability. This is because the $\mathrm{Fe}_{3} \mathrm{O}_{4}$ particles formed during milling could not impede grain growth. However, cryomilling of $\mathrm{Fe}-10 \mathrm{wt} \% \mathrm{Al}$ powders in liquid argon as well in liquid nitrogen resulted in nanocrystalline structures which were thermally stable at least up to $1223 \mathrm{~K}$. The enhanced thermal stability of the cryomilled Fe-10 wt $\%$ Al powders in liquid argon was attributed to the formation of $\gamma$ $\mathrm{Al}_{2} \mathrm{O}_{3}$ due to moisture condensation. The thermal stability of this alloy powder milled in liquid nitrogen was attributed to the formation of oxynitrides, or $\gamma-\mathrm{Al}_{2} \mathrm{O}_{3}$ and $\mathrm{AlN}$ particles during cryomilling in liquid nitrogen and to the solute segregation resulting in solute drag at the grain boundaries [219]. The $\mathrm{Al}_{2} \mathrm{O}_{3}$ and $\mathrm{AlN}$ particles act as fine dispersoids that stabilize the grain size via Zener pinning. However, only a small amount of the $\mathrm{Al}$ reacted with the $\mathrm{O}$ ans $\mathrm{N}$ to form oxides and 


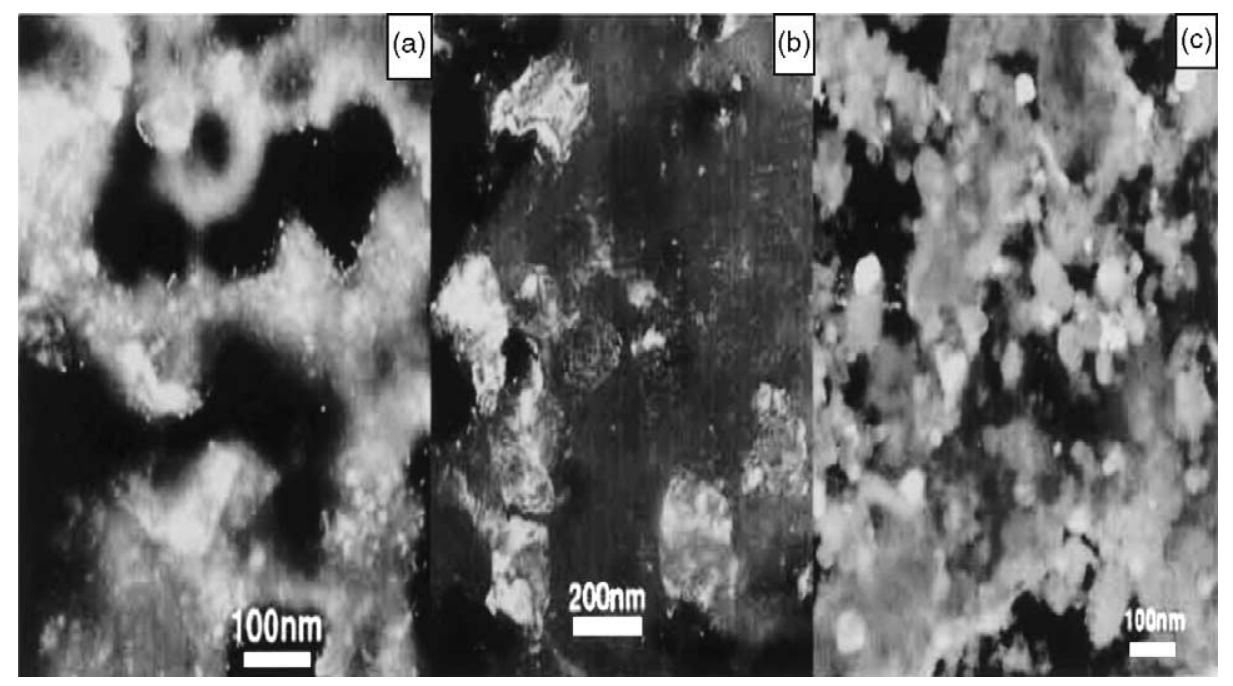

Fig. 29. TEM micrographs of $\mathrm{Zn}$ ball mill at room temperature for (a) $1 \mathrm{~h}$, (b) $3 \mathrm{~h}$ and (c) $18 \mathrm{~h}$ (reprinted from [213] with permission from Elsevier).

nitrides, and that the reminder of the $\mathrm{Al}$ was segregated to the grain boundaries. This solute segregation also contributed significantly to the thermal stability of the Fe-10 wt.\% Al [56].

\subsubsection{Mechanochemical synthesis}

This process involves mechanical activation of solid state displacement reactions in a ball mill. Thus, mechanical energy is used to induce chemical reactions. The chemical precursors typically consist of mixtures of oxides, chlorides and/or metals that react either during milling or during subsequent heat treatment to form a composite powder consisting of the dispersion of ultrafine particles within a soluble salt matrix. The ultrafine particle is then recovered by selective removal of the matrix phase through washing with an appropriate solvent. Mechanochemical synthesis is generally based on the following displacement reaction:

$$
\mathrm{A}_{x} \mathrm{C}+y \mathrm{~B} \rightarrow x \mathrm{~A}+\mathrm{B}_{y} \mathrm{C}
$$

where $\mathrm{A}_{x} \mathrm{C}$ and $\mathrm{B}$ are precursors, $\mathrm{A}$ is the desired product and $\mathrm{B}_{y} \mathrm{C}$ is a by-product of the reaction. This process has been used successfully to prepare nanoparticles of a number of materials, including transition metals [220,221], oxide ceramics (e.g. $\mathrm{Al}_{2} \mathrm{O}_{3}, \mathrm{ZrO}, \mathrm{ZnO}, \mathrm{PBT}$ (mixture of $\mathrm{PbO}, \mathrm{ZrO}_{2}$ and

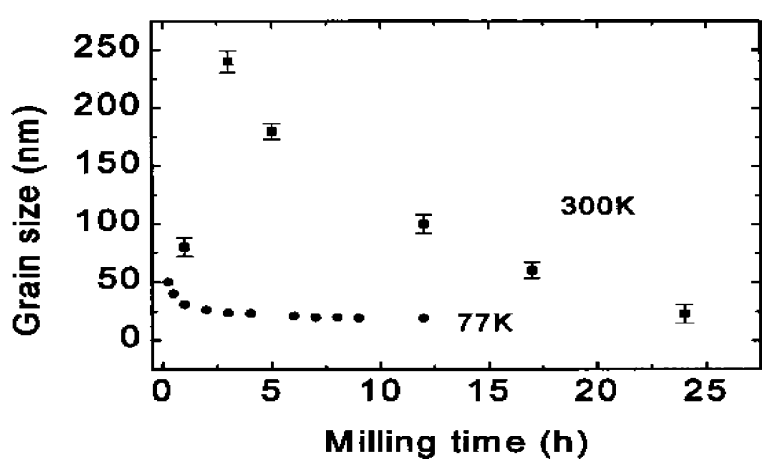

Fig. 30. The variation of grain size with milling time for $\mathrm{Zn}$ ball milled at room temperature and liquid nitrogen temperature (reprinted from [213] with permission from Elsevier). 
$\left.\mathrm{TiO}_{2}\right)$ ) [222-226], and oxide semiconductors (e.g. $\mathrm{SnO}_{2}$ ) [227]. For example, pure Ni nanoparticles of $10-20 \mathrm{~nm}$ can be synthesized by milling $\mathrm{NiCl}_{2}$ with $\mathrm{Na}$ in a mill for various times followed by washing with distilled water, and rinsing with methanol. Washed powders are subsequently dried under vacuum. The reaction takes place as follows [220]:

$$
\mathrm{NiCl}_{2}+2 \mathrm{Na} \rightarrow \mathrm{Ni}+2 \mathrm{NaCl}
$$

Zirconia can be synthesized by mechanochemical reaction of $\mathrm{ZrCl}_{4}$ with $\mathrm{MgO}$ in a vibratory mill under argon atmosphere, i.e.

$$
\mathrm{ZrCl}_{4}+2 \mathrm{MgO} \rightarrow \mathrm{ZrO}_{2}+2 \mathrm{MgCl}_{2}
$$

The milled powders were either washed, or first heat treated at $400-600{ }^{\circ} \mathrm{C}$ and then washed. Washed powders were then dried overnight at $100{ }^{\circ} \mathrm{C}$ [224]. Fig. 31a shows a TEM micrograph of as-milled

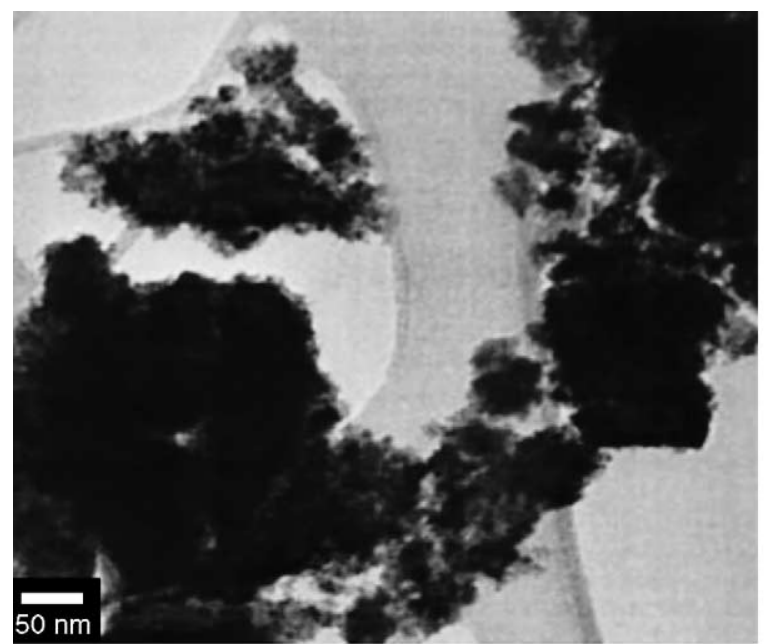

(a)

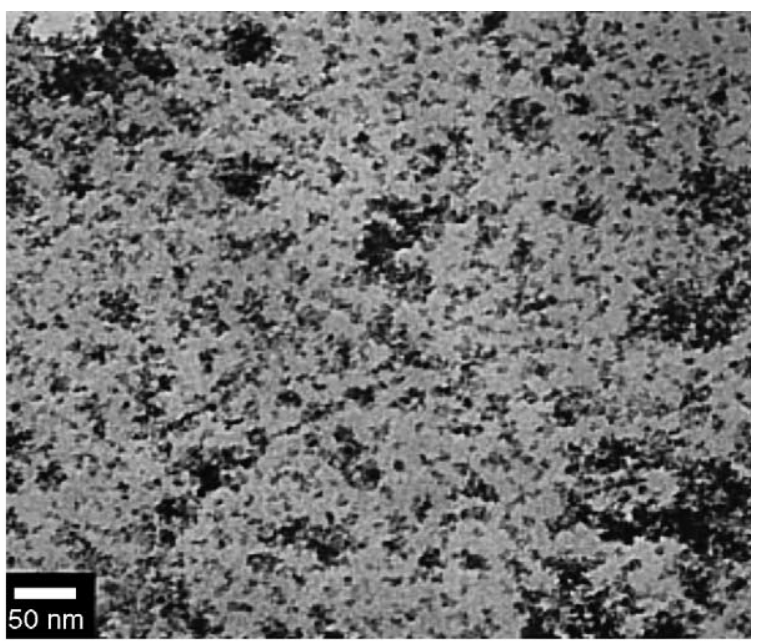

(b)

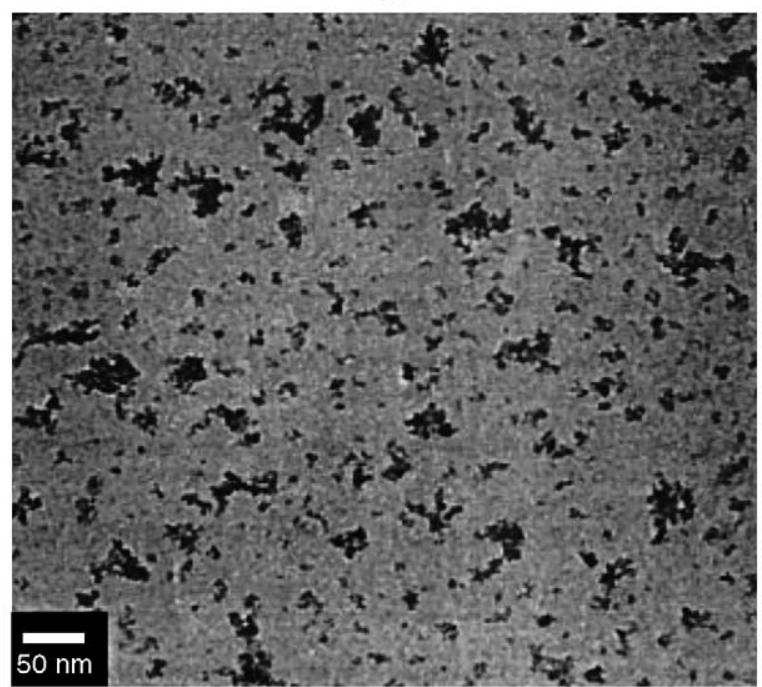

(c)

Fig. 31. TEM micrographs of (a) as-milled $\mathrm{ZrCl}_{4}+2 \mathrm{MgO}$ following water washing, (b) as-milled $\mathrm{ZrCl}_{4}+2 \mathrm{MgO}$ following heat treatment water washing and (c) as-milled $\mathrm{ZrCl}_{4}+2 \mathrm{MgO}$ following heat treatment water washing and thanol rinsing (reprinted from [224] with permission from Elsevier). 
$\mathrm{ZrCl}_{4}+2 \mathrm{MgO}$ following water washing. Apparently, the powder is comprised of relatively large agglomerates. No individual particles can readily be seen. The severity of agglomeration can be reduced by heat treating the as-milled powder at $500{ }^{\circ} \mathrm{C}$ followed by water washing (Fig. 31b). Additional ethanol rinsing following water washing leads to the formation of ultrafine $\mathrm{ZrO}_{2}$ particles (Fig. 31c). The high agglomeration of zirconia prepared by water washing of the as-milled powder is attributed to the effects of hydrogen bonding between surface hydroxyl groups formed through the adsorption of water molecules during washing. Heat treatment of the as-milled powder tends to reduce the degree of agglomeration by decreasing the surface reactivity of the zirconia particles towards water. Thus, fewer surface hydroxyl groups are formed during washing. The degree of agglomeration can be further reduced via ethanol rinsing. This is because surface hydroxyl groups are replaced by ethoxides which merely decompose during calcination without neck formation between particles [224].

\section{Fabrication of bulk nanocrystalline solids}

There is increasing scientific and industrial interest in the development of bulk nanocrystalline solids for laboratory mechanical testing and structural applications. Currently, the use of nanomaterials is still limited in industrial sectors because applications require the large-scale control of the synthesis of nanoparticles, and their subsequent processing into bulk nanostructures. In-situ compaction of IGC powders in the consolidation chamber at room temperature only yields a small disk of about 8-9 $\mathrm{mm}$ diameter with some internal pores [2]. Consolidation of nanopowders into a bulk material by conventional methods such as hot pressing, extrusion, and hot isostatic pressing for extended times can considerably coarsen the nanograins under the applications of both heat and pressure during compaction. Accordingly, techniques employing a very short heating duration such as shock wave compaction have been developed and achieved some success. These new methods are still in an embryonic stage of development for large-scale industrial applications. Apart from consolidation of nanopowders, severe plastic deformation (SPD) shows promise to deform bulk structures having coarser grains into submicron products with a mean grain size of about $100 \mathrm{~nm}$. SPD can produce large amounts of bulk samples with no residual porosity for mechanical testing. However, the final structures are metastable and therefore susceptible to grain growth at high temperatures. Currently, electrodeposition has been demonstrated to be an effective route to produce dense nanostructured sheets for the mechanical measurements. Its low cost, industrial applicability, versatility and high production rates have created increased interest in this technology for the deposition of nanocrystalline metals for industrial applications.

\subsection{Consolidation from nanocrystalline powders}

Most synthesizing methods produce nanocrystalline materials in a powder form as discussed in Section 3. There is a lack of bulk large dimension and pore-free nanocrystalline specimens for reliable mechanical testing to be carried out. Various standard compaction techniques frequently used for the consolidation of nanocrystalline powders include hot pressing [228,229], hot extrusion [230,231], cold isostatic pressing [232,233], and hot isostatic pressing (HIP) [232-235]. Consolidation of nanocrystalline powders into fully dense material must not alter the nanostructure in order to retain the unique properties of fine particles. Consolidation of nanopowders under the applications of temperature and pressure generally can yield nearly fully dense compacts [236]. However, recrystallization and graingrowth occur readily during high temperature consolidation of nanocrystalline powders, particularly 
those prepared from the MA process. In this regard, shock wave compaction is attractive because it can be used to fully densify powdered materials without inducing activated microstructural and compositional changes $[237,238]$. The characteristic features of the shock-wave deformation are high pressure (e.g. $>5 \mathrm{GPa}$ ) and moderate temperature, generated and dissipated on a very short period of time (1$10 \mu \mathrm{s})$ and extremely high rate of deformation $\left(10^{6}-10^{7} \mathrm{~s}^{-1}\right)$ [237]. Thus, shock compaction can produce high compressive stresses during short time periods, thereby heavily deforming the particles, melting the surfaces, and producing a fully dense compact without grain growth. Various guns and explosive detonation methods have been developed and used for shock compaction and shock synthesis of bulk nanocrystalline materials [238-245].

In another approach, Alexandrov et al. attempted to use high pressure torsion (HPT) to produce nearly fully dense disks of $\mathrm{Cu}$ and $\mathrm{Al}$ as well as $\mathrm{Cu}$-based nanocomposites from MA nanopowders. HPT is normally used for refining coarser-grained bulk materials with sizes of several micrometers to the submicron level. Grain size was defined during the consolidation by plastic torsional straining. However, the as-processed materials are very brittle, possibly resulting from the oxidation of the initial nanopowders during straining [246].

\subsection{Severe plastic deformation}

There are two major procedures for severe plastic deformation processing, namely high-pressure torsion and equal-channel angular pressing (ECAP). In the HPT process, a sample in the form of a disk is subjected to very large shear torsion straining under the applied high pressure of several GPa at room temperature. One of the sample holders rotates and surface friction deforms the material by shear (Fig. 32a). In ECAP, the sample is pressed through a die in which two channels, equal in cross section, intersect at an angle $\Phi$, with an additional angle $\Psi$ defining the outer arc of curvature where the two channels intersect (Fig. 32b). The sample is pressed through the die using a plunger and subjected to shear as it passes through the shearing plane at the intersection of two channels. As deformed dimensions are identical to the initial ones, thus it is possible to repeat this process for many cycles to accumulate large plastic strain. In this regard, ECAP is more attractive than HPT because it can be used to produce not only laboratory samples but bulk submicron-grained billets for industrial applications. Both of these procedures are known to induce a high density of dislocations that
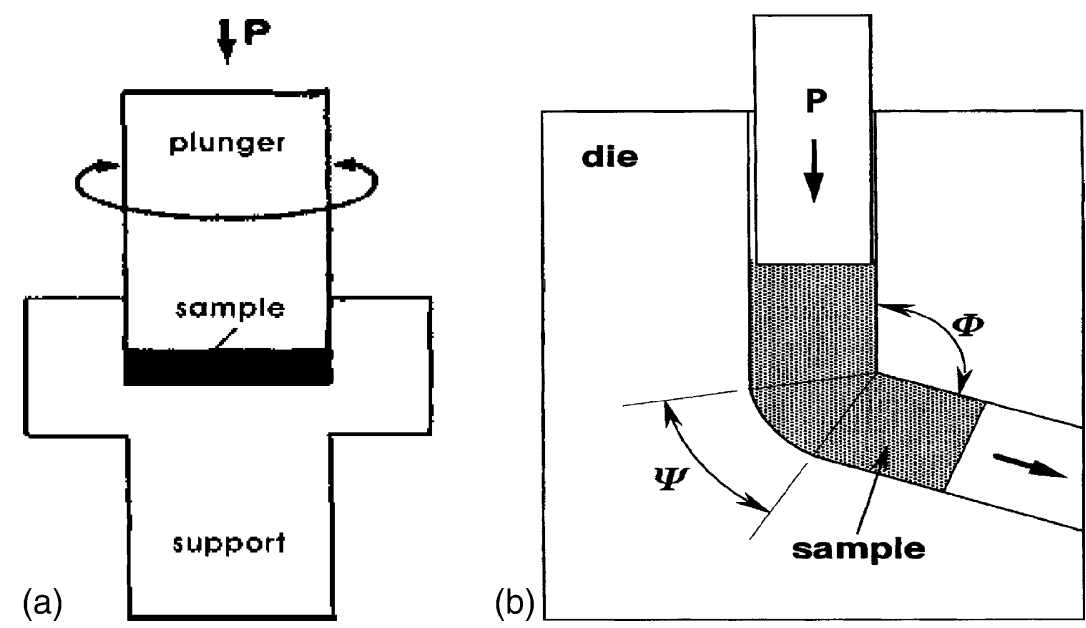

Fig. 32. Schematic diagrams showing severe plastic deformation methods of forming materials with submicron grain sizes: (a) HPT and (b) ECAP. 
arrange subsequently into metastable subgrains of high-angle boundaries. In pure metals, the application of HPT usually results in the formation of an ultrafine-grained structure with an average size of about $100 \mathrm{~nm}$ and the application of ECAP yields a grain size of 200-300 nm [247]. A particular cry stall ographic texture is formed in ECAP samples [248]. It has been demonstrated that ECAP can successfully produce numerous submicron-grained metallic materials including Ni [249], $\mathrm{Cu}$ [250,251], $\mathrm{Fe}-\mathrm{Cu}$ alloy [252], Ti alloy [253], Al and its alloys [250,254-261]. The submicronsized grains of $\mathrm{Al}$ and its alloys processed by ECAP are generally unstable at temperatures above $\sim 500 \mathrm{~K}$ except when the precipitates are present. The precipitate particles can inhibit the grain growth of selected Al-alloys. In this case, the grains remain within the submicrometer range up to temperatures in the vicinity $\sim 700 \mathrm{~K}[255,258]$. The stability of ultrafine grains due to precipitate formation at elevated temperatures can lead to the occurrence of superplasticity in Al-2024, Al-7034 and $\mathrm{Al}-(3-4) \mathrm{Mg}-0.2 \mathrm{Sc}$ alloys processed by ECAP $[258,259,262]$.

\subsection{Electrodeposition}

Compared with mechanical consolidation and SPD, the advantages of electrodeposition for forming dense nanocrystalline materials are: (a) low cost and industrial applicability, as it involves little modification of existing electroplating technologies, (b) simple operation, as the electrodeposition parameters can be easily tailored to meet the required crystal grain size, microstructure and chemistry of products, (c) versatility, as the process can produce a wide variety of pore free materials and coatings and (d) high production rates. Nanocrystalline deposits are formed on a cathode surface during plating by properly controlling the electrodeposition parameters, e.g. bath composition, temperature, $\mathrm{pH}$, etc. Extensive studies have been conducted on the use of pulse plating [263273] and DC plating for these purposes [274-276]. Pulse plating is particularly attractive because it can be used to produce finer-grained structures and coatings that are not obtainable by DC plating. In pulse plating, current is imposed in a repetitive square wave with the following controlling parameters: peak current density, pulse on-time and pulse-off-time. El-Sherik and Urb [263,264] studied the process for producing nanocrystalline $\mathrm{Ni}$ having grain sizes in the range $10-40 \mathrm{~nm}$ and thickness of $\sim 40-50 \mu \mathrm{im}$ by pulse electrodeposition from a modified Watts-type bath. The solution consists of nickel sulfate, nickel chloride, boric acid and saccharin inhibitor $\left(\mathrm{C}_{7} \mathrm{H}_{4} \mathrm{NO}_{3} \mathrm{~S}\right)$. The deposits generally have an equiaxed structure with fairly narrow grain size distribution. To achieve a significant grain refinement, the pulse-off times must be longer than pulse-on times. And the grain refiner such as saccharin is needed to retard the grain growth of Ni deposits. In the absence of saccharin, large crystals in the micrometer range with dull and rough appearances are obtained. However, sulfur and carbon impurities content tend to increase with increasing saccharin content in the bath until saturation occurs. These impurities originate from the chemicals and inhibitor used for the bath [273], and may degradate the mechanical properties of electrodeposits. For example, sulfur is known to be a critical impurity causing embrittlement [277]. The electrodeposits are also found to exhibit a texture structure, depending on the bath chemistry. The preferred orientation of the deposits progressively changed from

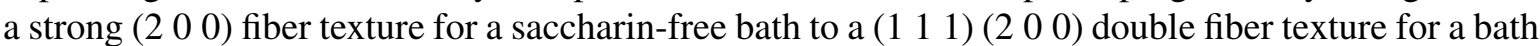
containing $10 \mathrm{~g}^{-1}$ saccharin [264]. It has been known for many years that saccharin causes an $\left(\begin{array}{lll}1 & 1 & 1\end{array}\right)$ orientation [278].

$\mathrm{Ni}$ deposits are of particular interest because they are commonly used as test samples for mechanical characterization. Fig. 33a and b show respective TEM bright and dark field micrographs of nanocrystalline Ni deposited from a modified Watt's bath. The first four rings of the electron diffraction pattern represent $\left(\begin{array}{lll}1 & 1 & 1\end{array}\right),\left(\begin{array}{lll}2 & 0 & 0\end{array}\right),\left(\begin{array}{lll}2 & 2 & 0\end{array}\right)$ and $\left(\begin{array}{lll}3 & 1 & 1\end{array}\right)$ planes of the nanocrystalline $\mathrm{Ni}$, respectively (Fig. 33c). The grain size distribution is determined from the dark field micrographs, 

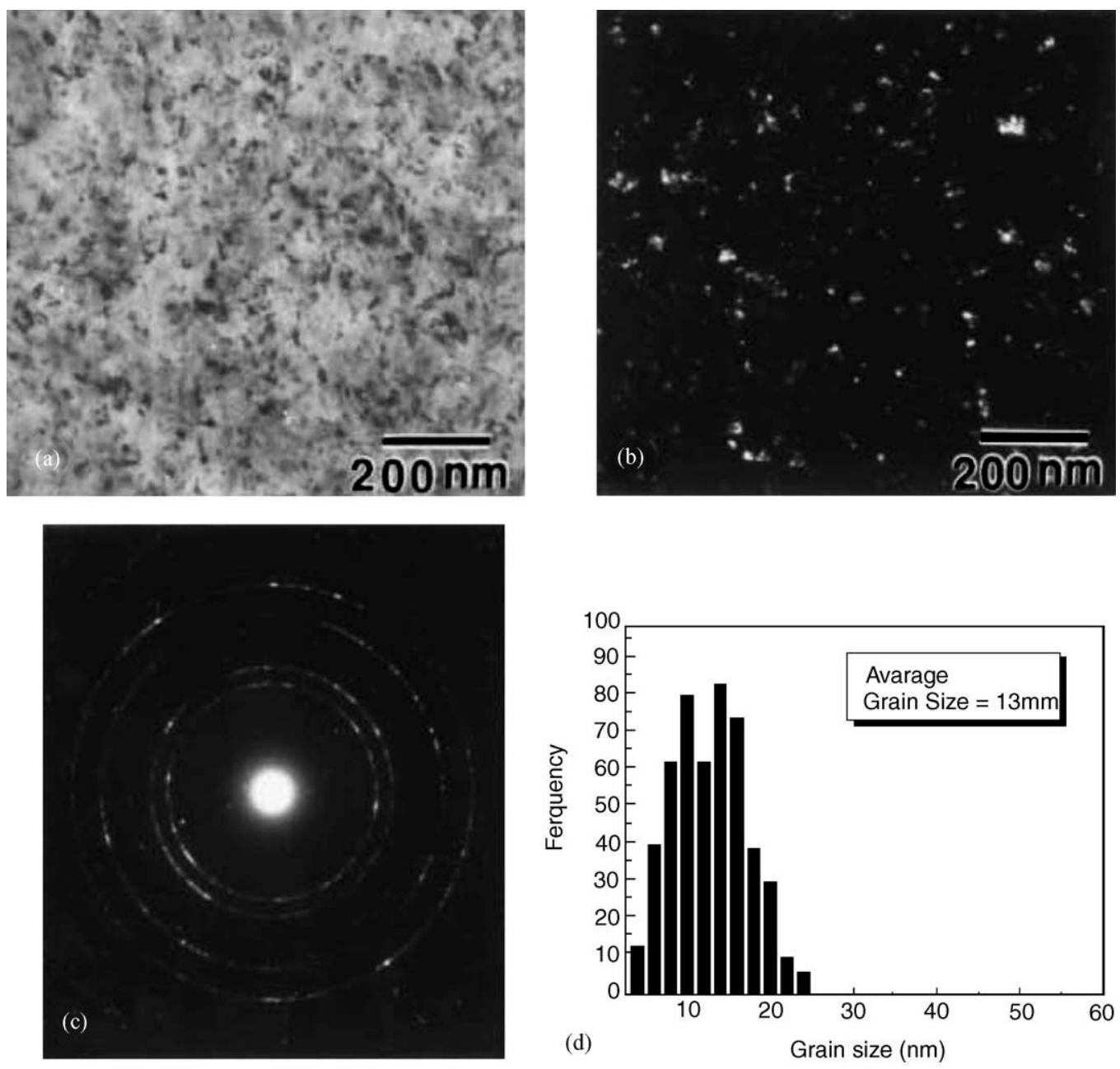

Fig. 33. (a) Bright field and (b) dark filed TEM micrographs with (c) electron diffraction pattern and (d) grain size distribution of electrodeposited nanocrystalline Ni (reprinted from [271] with permission from Elsevier).

Table 5

Summary of the mechanical properties of conventional and nanocrystalline nickel (reprinted from [279] with permission from Elsevier)

\begin{tabular}{|c|c|c|c|}
\hline Property & $\mathrm{Ni} 10 \mu \mathrm{m}$ & Ni $100 \mathrm{~nm}$ & $\mathrm{Ni} 10 \mathrm{~nm}$ \\
\hline Yield strength $(\mathrm{MPa})\left(25^{\circ} \mathrm{C}\right)$ & 103 & 690 & $>900$ \\
\hline Ultimate tensile strength $(\mathrm{MPa})\left(25^{\circ} \mathrm{C}\right)$ & 403 & 1100 & $>2000$ \\
\hline Tensile elongation $(\%)\left(25^{\circ} \mathrm{C}\right)$ & 50 & $>15$ & 1 \\
\hline Elongation in bending $(\%)\left(25^{\circ} \mathrm{C}\right)$ & - & $>40$ & - \\
\hline Modulus of elasticity (GPa) $\left(25^{\circ} \mathrm{C}\right)$ & 207 & 214 & 204 \\
\hline Vickers hardness $\left(\mathrm{kg} / \mathrm{mm}^{2}\right)$ & 140 & 300 & 650 \\
\hline Work hardening coefficient & 0.4 & 0.15 & 0 \\
\hline Fatigue strength $(\mathrm{MPa})\left(10^{8}\right.$ cycles/air/ $\left.25^{\circ} \mathrm{C}\right)$ & 241 & 275 & - \\
\hline Wear rate (dry air, pin on disk) $\left(\mu \mathrm{m}^{3} / \mu \mathrm{m}\right)$ & 1330 & - & 7.9 \\
\hline Coefficient of friction (dry air, pin on disk) & 0.9 & - & 0.5 \\
\hline
\end{tabular}


showing an average grain size of $13 \mathrm{~nm}$ (Fig. 33d) [271]. Typical mechanical properties of conventional microcrystalline $\mathrm{Ni}(10 \mu \mathrm{m})$ and electrodeposited nanocrystalline $\mathrm{Ni}$ with grain size of 10 and $100 \mathrm{~nm}$ are summarized in Table 5 [279]. It is obvious that nanocrystalline Ni samples exhibit superior monotonic and cyclic strengths, hardness, and excellent wear resistance but poorer tensile ductility compared to conventional microcrystalline Ni. Moreover, nanocrystalline Ni electrodeposits do not show significant reduction in Young's modulus compared to nanocrystalline materials prepared by consolidation of nanopowders. Thus, electrodeposited nanocrystalline Ni coatings with good mechanical properties lead to practical uses as integral sleeve materials for nuclear steam generator tubing [280].

\section{Mechanical behavior}

Conventional polycrystalline metals and alloys show an increase in yield strength $\left(\sigma_{\mathrm{y}}\right)$ with decreasing grain size $(d)$ according to the well-known Hall-Petch $(\mathrm{H}-\mathrm{P})$ equation:

$$
\sigma_{\mathrm{y}}=\sigma_{0}+k d^{-1 / 2}
$$

where $\sigma_{0}$ is friction stress resisting the motion of gliding dislocation, and $k$ is the Hall-Petch slope, which is associated with a measure of the resistance of the grain boundary to slip transfer. In analogy, hardness $\left(H_{\mathrm{v}}\right)$ can be related to the grain size by

$$
H_{\mathrm{v}}=H_{0}+k_{\mathrm{H}} d^{-1 / 2}
$$

where $H_{0}$ and $k_{\mathrm{H}}$ are cosnstants. Hardness is a measure of the resistance of a material to plastic deformation under the application of indenting load. The $\mathrm{H}-\mathrm{P}$ effect in conventional coarse-grained materials is attributed to the grain boundaries acting as efficient obstacles to dislocations nucleated mostly from Frank-Read sources. Consequently, a dislocation pileup can be formed against a grain boundary inside a grain.

By decreasing the grain size of metals down to the order of a few tens of nanometers, the H-P slope remains positive but with a smaller value [281-284]. At ultra-fine grained sizes below ca. $20 \mathrm{~nm}$, a reversed softening effect or negative $\mathrm{H}-\mathrm{P}$ relation is observed for some metals [285-289]. The interpretation of this inverse $\mathrm{H}-\mathrm{P}$ effect is still subjected to debate. Several factors such as grainboundary sliding, creep diffusion, triple junctions, pores and impurities could contribute to inverse $\mathrm{H}-$ P relation in metals and alloys [285,286,290-292]. Takeuchi [293] summarizes the hardness-grain size results of sereral workers for nanocrystalline $\mathrm{Cu}$ and Pd samples in Fig. 34a and b. These research groups include Choksi et al. [286], Nieman et al. [282], Fougere at al. [288] and Sanders et al. [69]. Apparently, the $\mathrm{H}-\mathrm{P}$ slope for $\mathrm{Cu}$ varies gradually from positive, zero (no obvious grain size dependence) to negative. Careful examination of these figures reveal that a distinct deviation from the $\mathrm{H}-\mathrm{P}$ relation i.e. negative slope is observed for both the IGC $\mathrm{Cu}$ and $\mathrm{Pd}$ samples prepared by Choksi et al. In contrast, data results from other workers only reach a plateau regime as the grain size decreases. The $\mathrm{Cu}$ and $\mathrm{Pd}$ samples used in the plots are prepared from inert gas condensation (IGC) powders followed by compaction. Consolidated IGC samples may contain internal impurities and defects. The cleanliness of the vacuum environment, gas purity and outgassing rate of the vacuum system determine the presence of impurities in the material obtained [294]. Moreover, pores and other flaws developed in the consolidated IGC powders are detrimental to the mechanical properties such as a reduction of the modulus $[2,69]$. Though all the data results presented in Fig. 34 are derived from the IGC samples, but the impurity chemistries and contents of IGC samples differ from one research group 

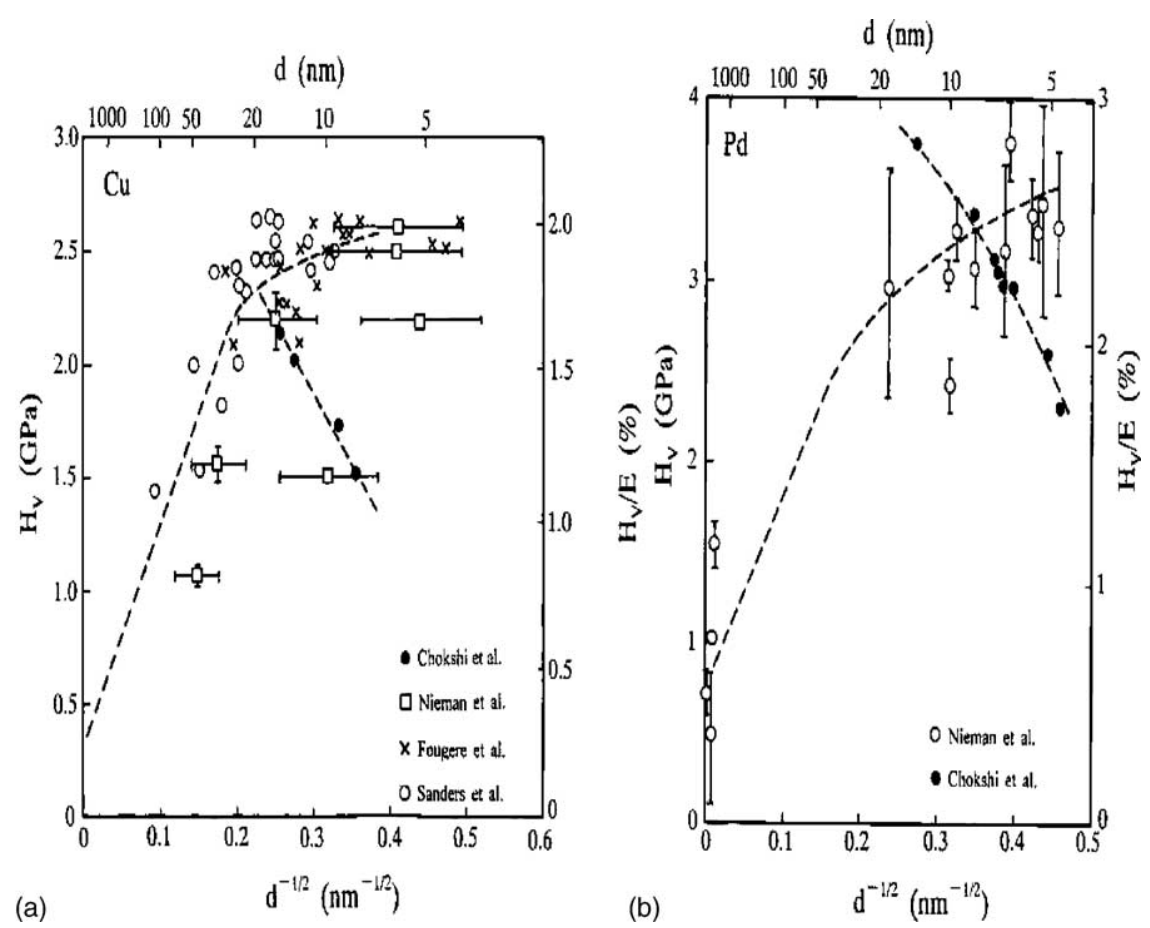

Fig. 34. Hall-Petch plots of the hardness of: (a) nanocrystalline $\mathrm{Cu}$ and (b) nanocrystalline Pd (reprinted from [293] with permission from Elsevier).

to another. Consequently, the production and consolidation of IGC powders will greatly affect the mechanical properties of nanocrystalline metals. For pore free and dense electroplated Ni samples, El-Sherik et al. also observed a deviation from H-P relation. A plateau is found in the hardness versus grain size curve when the grain sizes are less than $20 \mathrm{~nm}$ (Fig. 35) [284]. This raises the issue of whether inverted $\mathrm{H}-\mathrm{P}$ behavior is inherited from the intrinsic effect of nanograin size or resulted from extrinsic defects introduced in the samples during fabrication.

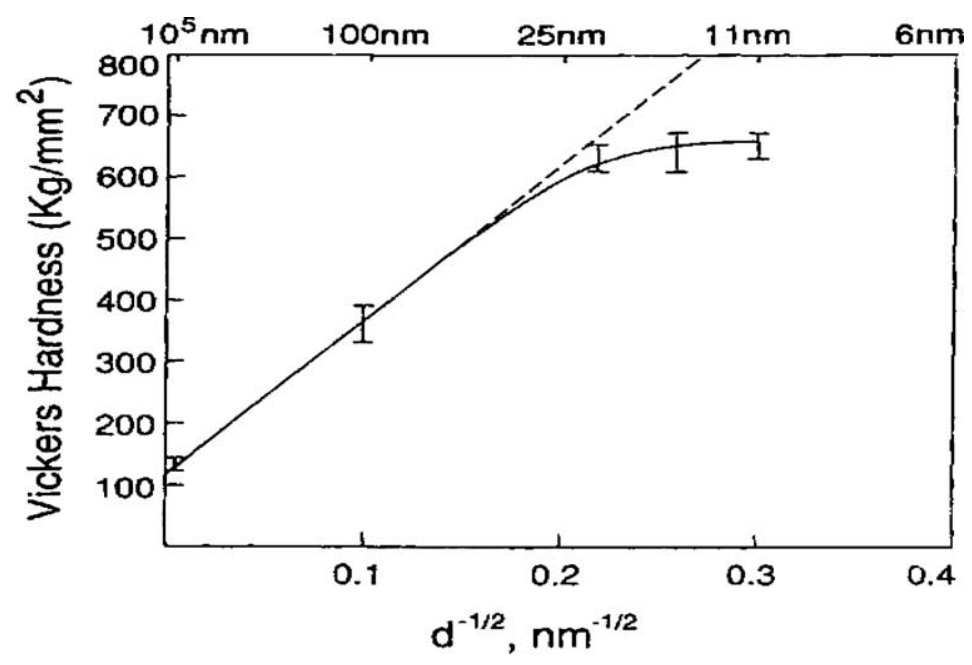

Fig. 35. Hall-Petch plot of the hardness of nanocrystalline Ni prepared by electrodeposition (reprinted from [284] with permission from Elsevier). 
From this, it appears that conventional dislocation mechanisms cease to function when the grain sizes are below a critical value. This is because the size of a Frank-Read source cannot exceed the grain size. In this case, pile-up of the dislocations against grain boundaries would not occur. Mesoscopic theoretical modeling and computer simulations aiming at understanding of the basic mechanisms underlying the inverse $\mathrm{H}-\mathrm{P}$ relation have been carried out by several researchers. As nanocrystalline metals contain a high fraction of atoms at interfaces that contain free volumes, there is a widespread speculation that deformation occurs primarily at the grain boundaries. A number of models have been proposed to explore the grain boundary associated deformation mechanisms for nanocrystalline metals, e.g. grain boundary sliding, grain boundary rotation, grain boundary diffusion, triple junction effect, etc. Hahn et al. $[295,296]$ have proposed a phenomenological mesoscopic model to explain the inverse $\mathrm{H}-\mathrm{P}$ relation of nanostructured materials. The inverse $\mathrm{H}-\mathrm{P}$ effect is considered to be associated with the sliding of grain boundaries. In this approach, a network of grain boundaries with thickness $\delta$ is surrounded essentially by non-deforming grains with a polyhedral structure (Fig. 36). A grain boundary is divided into a number of atomic-scale ensembles that contain free volume. Sliding occurs by the movement of boundary volume elements of size $\delta^{3}$ on the grain boundaries. As sliding occurs, an internal shear stress distribution $f\left(\tau_{\mathrm{i}}\right)$, which opposes the sliding, is created along the grain boundaries. To produce substantial sliding on a mesoscopic scale, two or more grains must cooperate to form a planar interface which by further interconnection with other planar interfaces will lead to long-range sliding. Once a planar interface is formed the localized sliding shears can result in mesoscopic sliding over the dimensions of many grains. To analyze the sliding effect mathematically, the grains take the shape of a rhombic dodecahedron (Fig. 36). The internal stress $\tau_{\mathrm{i}}$, is finally deduced as:

$$
\begin{aligned}
& \tau_{\mathrm{i}}=G\left[\frac{d_{1}}{d}\left(1-\frac{d_{2}}{d}\right)\right]^{1 / 2} \text { for } d \geq d_{2} \\
& \tau_{\mathrm{i}}=0 \text { for } d<d_{2}
\end{aligned}
$$

where $G$ is the shear modulus. This model predicts a critical grain size $\left(d_{2}\right)$ where the internal stress falls to zero and hence sliding becomes very rapid. To correlate with the observations of the microhardness tests, the internal stress in Eq. (E17) is related by the hardness parameter $\left(\tau_{\mathrm{i}}=\right.$ $C H_{\mathrm{v}}$, where $C$ is a constant). An empirical equation $H_{\mathrm{v}}=H_{0}-m / d^{1 / 2}$ is obtained accordingly; it
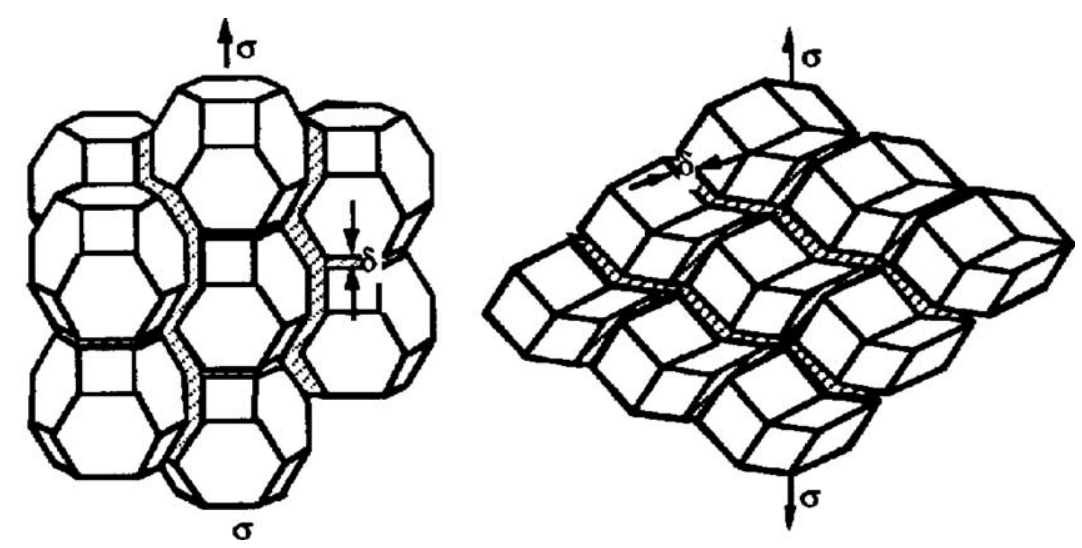

Fig. 36. Tetrakai decahedral (left) and rhombic dodecahedral (right) shapes in an idealized grain arrangement with grain boundaries separating the grains (reprinted from [295] with permission from Elsevier). 
exhibits an inverse H-P relationship. This model clearly is conststent with the hypothesis that the negative $\mathrm{H}-\mathrm{P}$ effect in consolidated IGC Cu resulted from the grain-boundary sliding, though Chokshi et al. [286] attributed the negative slope to Coble creep diffusion (creep rate scales as $D_{\mathrm{GB}} / d^{3}$ where $D_{\mathrm{GB}}$ is the grain-boundary diffusion coefficient). The drawback of this model is that many adjustable parameters used are difficult to quantify, making real testing of the model difficult.

As contamination-free and dense nanocrystalline metals with grain size smaller than $20 \mathrm{~nm}$ are not available at present, large scale molecular dynamics (MD) modeling proves to be a powerful tool to simulate the interfacial structure and mechanical deformation mechanism of nanocrystalline materials [297-308]. Simulations can provide the atomic level details and deformation structures that are not accessible by experimental routes. The simulations are strongly dependent on the appropriate selection of empirical models for atomic interactions and systems with realistic grain structures. Most simulations are performed on fully dense fcc metals (e.g. $\mathrm{Ni}, \mathrm{Cu}$ and $\mathrm{Pd}$ ) with their adjacent grains separated by high angle boundaries, and free from impurities. Three-dimensional (3D) nanocrystalline grains are either created by filling the space according to the Voronoi construction with randomly nucleated seeds and crystallographic orientations [297-299], or by crystallization from a melt [306,307]. Van Swygenhoven and coworkers reported that the grain boundary region of nanocrystalline $\mathrm{Cu}$ and $\mathrm{Ni}$ constructed from a Voronoi model has a high degree of structural order that is not fundamentally different from the grain boundaries in polycrystals. In the simulation, a tight binding potential in the Parrinello-Rahman approach was employed. Periodic boundary conditions and fixed orthorhombic angles were imposed on the computational cell. On the other hand, Keblinski et al. proposed the formation of highly disordered or amorphous 'glue-like' grain-boundary structures. MD simulations using the Stillinger-Weber three-body potential were used to synthesize fully dense nanocrystalline silicon with a grain size up to $7.3 \mathrm{~nm}$ by crystallization from the melt [306]. A nanocrystalline structure wass generated in modeling where a liquid was solidified in the presence of randomly oriented crystalline seeds. The system was then quenched, and the liquid crystallized on the seeds, thus yielding a nanocrystalline structure [306]. In this case, high atomic mobility takes place easily at less-perfect atomic packing at nano-grain boundaries.

MD simulations are particularly useful to predict the nanocrystalline plasticity in the grains with diameters below $10 \mathrm{~nm}$. Such simulations are commonly performed in uniaxial tension under high load and high strain rate conditions. In recent simulations on the tensile deformation of nanocrystalline $\mathrm{Ni}$ with grain sizes of 3-12 $\mathrm{nm}$, Van Swygenhoven et al. indicated that grain boundary sliding is the primary deformation mechanism for $\mathrm{Ni}$ with grain size $<10 \mathrm{~nm}$. The sliding is triggered by atomic shuffling and to some extent by stress-assisted free volume migration. Above $10 \mathrm{~nm}$, intergrain sliding and dislocation emission from the grain boundaries could take place. The grain boundaries are considered to be acting as sources and sinks for the dislocation activity [300,302]. Schiotz et al. also performed MD simulations of deformation in three-dimensional $\mathrm{Cu}$ nanocrystalline samples in the grain size range $3.28-13.2 \mathrm{~nm}$ at 0 and $300 \mathrm{~K}$, respectively [304,305]. The grains were grown according to the Voronoi construction. The nanocrystal contained approximately $10^{5}$ atoms arranged in 8-64 grains. An interaction potential approach based on the effective-medium theory was adopted to calculate the forces acting between the atoms in the simulations. The deformation was applied by stretching the nanocrystal in one direction. The movement of individual atoms was calculated, and their positions were recorded. Thus, the deformation strain could be observed at every step of the process. The average stress in the sample as a function of the amount of deformation was calculated. Fig. 37 shows typical MD stress-strain curves for nanocrystalline $\mathrm{Cu}$ with various grain sizes $\leq 13.2 \mathrm{~nm}$ at $300 \mathrm{~K}$. The variations of flow and yield stresses with grain sizes at 0 and $300 \mathrm{~K}$ are shown in Fig. 38. Simulations yield a negative H-P relation at both temperatures in the absence of porosity. The main deformation mechanism is localized sliding of atoms in the grain boundaries, leading to a 


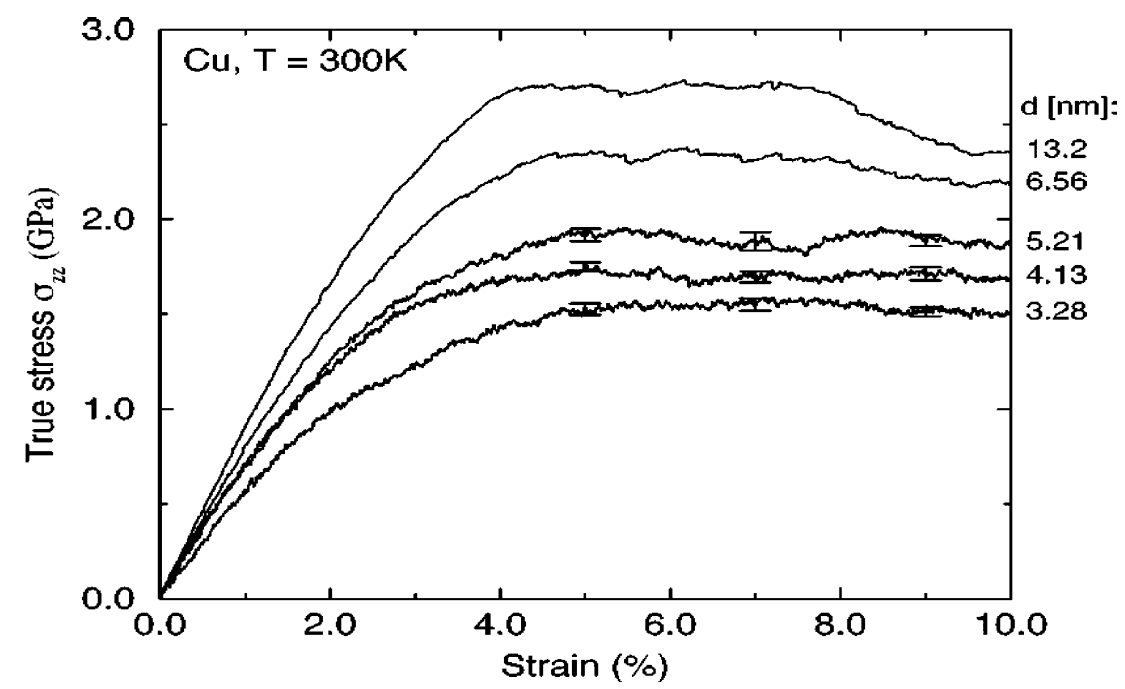

Fig. 37. Atomic-scale simulation of the stress-strain curves for nanocrystalline copper at $300 \mathrm{~K}$ for varying grain size (reprinted from [305] with permission from The American Physical Society).

reversed H-P relation in defect-free copper. Further, grain boundary sliding can occur without any thermally activated process at $0 \mathrm{~K}$, and driven by high stress only [304,305]. From the MD simulations of Swygenhoven et al. and Shiotz et al., it appears that no dislocation activity exists below a critical grain size of $10 \mathrm{~nm}$. Plastic deformation takes place entirely by means of grain boundary sliding. Sliding of the grain boundaries in porosity-free nanocrystalline metals seems to be associated with a reduction in the elastic moduli of disordered grain boundary materials. Grain boundaries are considered elastically softer than the grain interiors of nanocrystalline metals [305]. There is no direct experimental evidence for the grain-boundary sliding in nanocrystalline materials with grain size $<10 \mathrm{~nm}$.

It should be noted that MD simulations of the deformation of nanocrystalline materials are performed at high loads, and under extremely high strain rates, e.g. on the order of $\sim 10^{8} \mathrm{~s}^{-1}$ [305]. Such high strain rates can cause serious plastic deformation in materials during practical tensile tests.

Grain size d $(\mathrm{nm})$

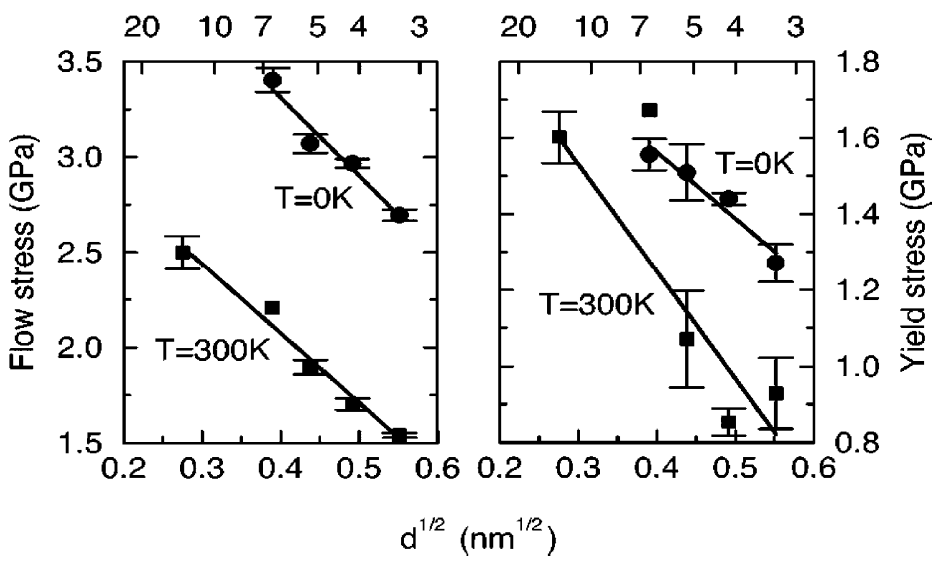

Fig. 38. Atomic-scale simulation of the Hall-Petch relationship between the grain size and both the yield- and flow stress of copper at 0 and 300K (reprinted from [305] with permission from The American Physical Society]. 
The MD simulations also have certain limitations. The time and size scales that can be studied with the MD method are restricted. These impose constraints on the number or the size of the grains to be simulated. The small number of grains compared with real samples may induce anomalies in the measured properties. Further, the deformation can only be initiated at early stages, thus extremely high strain rates are needed to get any reasonable deformation within the available time [299]. In this respect, simulations related to time-dependent deformation such as creep become difficult. Keblinski et al. have attempted to use MD simulation to study diffusion creep of silicon [307]. They reported that the total creep strains that can be simulated are rather small, in the order of $1-2 \%$. Another limitation of MD modeling is the absence of damage evolution and fracture behaviors of materials. Therefore, MD modeling of the deformation of nanocrystalline materials is incomplete and still subject to criticism. High-speed and high-performance supercomputers have been used increasingly in mathematical modeling; it is possible to perform simulations on nanocrystalline samples with larger numbers of both atoms and grains for longer times. Nevertheless, predictions from MD simulations of nanocrystalline structure and plasticity are complementary to the results of actual experimental measurements.

In addition to MD simulations, analytical modeling of the size and scale effects associated with elastic-plastic behavior of nanocrystalline materials has become an important research area and provided many insights into scale-dependent deformation mechanisms [309-312]. In the analytical modeling, nanocrystalline material is essentially considered as a composite comprising of the interior grains and grain boundaries. Additional factors relating to the structure and elastic-plastic deformation of materials are incorporated into the formulations by individual researchers according to the simulation needs. For example, Kim and Bush [309] have presented numerical work on the modeling of grain-size-dependent elastic modulus of nanocrystalline materials. They developed a phase mixture model in which nanocrystalline materials are considered to be a composite of the crystalline and the intercrystalline grain boundary phases (triple line junction and quadratic node). Moreover, porosity was also included in their numerical modeling. The simulation predicts the elastic modulus of nanocrystalline materials decreases with a decrease of the grain size and the decrement is relatively large at grain sizes below $10 \mathrm{~nm}$. The effect of porosity, however, is substantially greater than the grain size effect. Since nanocrystalline materials usually contain some porosity and the decrement of the elastic modulus due to the porosity is much more significant than that due to the grain size effect. The calculated values of Young's modulus for the $\mathrm{Cu}, \mathrm{Fe}$ and Pd specimens correlate reasonably with the experimental data. They then used the phase mixture model to describe the plastic deformation of nanocrystalline materials [310]. The mechanical properties of the crystallite were represented by a unified viscoplastic constitutive concept based on the evolution of dislocation density. The total strain rate of the crystallite was calculated by the summation of the contributions of dislocation, boundary diffusion and lattice diffusion mechanism. The deformation mechanism for the grain boundary was modeled as a diffusional flow of the matter through the grain boundary. This model can be used to explain the breakdown of the Hall-Petch relation with decreasing grain size and the rate dependence of the deformation behavior. In another approach, Sharma and Ganti attempted to evaluate the elastic properties of nanocrystalline materials as function of grain size by taking grain boundary sliding into consideration [312]. They treated nanocrystalline material as a two-phase system consisting of crystalline matrix phase and ellipsoidal grain boundary phase. The proposed analytical model agrees reasonably well with the experimental results for nanocrystalline copper and palladium. Grainboundary sliding appears to have only a minor effect on the apparent elastic modulus. Meyers and coworkers [311] also treated the nanocrystalline material as a composite, comprised of the grain interior, with flow stress $\sigma_{\mathrm{fG}}$ and grain boundary work-hardened layer, with flow stress $\sigma_{\mathrm{fGB}}$. Four principal factors contribute to grain-boundary strengthening. These include: (a) the grain boundaries 
act as barriers to plastic flow, (b) the grain boundaries act as dislocation sources, (c) elastic anisotropy causes additional stresses in the grain-boundary surroundings, and (d) multislip is activated in the grain-boundary regions, whereas grain interiors are initially dominated by single slip. From these, several parameters are grouped into $\kappa$ during the derivation of the following equation:

$$
\sigma_{\mathrm{y}}=\sigma_{\mathrm{fG}}+8 \kappa\left(\sigma_{\mathrm{fGB}}-\sigma_{\mathrm{fG}}\right) d^{-1 / 2}-16 \kappa^{2}\left(\sigma_{\mathrm{fGB}}-\sigma_{\mathrm{fG}}\right) d^{-1}
$$

For large grain size in the micrometer range, the $d^{-1 / 2}$ term dominates and a Hall-Petch relationship is obtained. The Hall-Petch slope, $k$, is equal to:

$$
k=8 \kappa\left(\sigma_{\mathrm{fGB}}-\sigma_{\mathrm{fG}}\right)
$$

As the grain size is decreased, the $d^{-1}$ term becomes progressively dominant. Thus, the decrease of the Hall-Petch slope in the nanocrystalline domain is explained and corresponds to a grain size for which the thickness of the work hardened layer is equal to one half of the grain diameter. This analytical prediction shows a good correlation with experimental results for $\mathrm{Cu}$ and $\mathrm{Fe}$.

Recently, rotational deformation occurring through motion of grain boundary disclination dipoles has been suggested as a deformation mechanism contributing to plastic flow in nanocrystalline materials [313-316]. Subsequently, there exists a cooperative action of grain boundary sliding and grain rotation during deformation. The rotational deformation is believed to be associated with the motion of dipoles of grain boundary disclination (Fig. 39) [313]. A disclination is a line defect characterized by a rotation of the crystalline lattice around its line. A disclination dipole consists of two disclinations causing crystal lattice rotation between them. It has been confirmed experimentally in mechanically loaded nanocrystalline metals and thin films [314,317]. Another interesting behavior of nanocrystalline metals associated with the grain boundary sliding accompanied by grain rotation is superplasticity. It is defined as the ability of some materials to exhibit extremely large extensibility, often without the formation of a neck prior to fracture. A fine-grained equiaxed structure microstructure (grain size $\leq 10 \mu \mathrm{m}$ ) is required for superplasticity behavior in microcrystalline materials at elevated temperatures. Moreover, the structure must be highly resistant to grain growth at the temperatures concurrent with superplastic deformation. Fine-grain size at elevated temperatures in microcrystalline solids can be retained in two-phase structure materials or in alloys having a fine dispersion of second phase particles. Microscopic examination of superplastically deformed alloys shows that extensive grain boundary sliding, accompanied by considerable grain rotation, occurs during superplasticity [318]. It is noted that the thermally activated grain-boundary sliding in superplasticity is accommodated by a mass transport diffusion process. The phenomenological

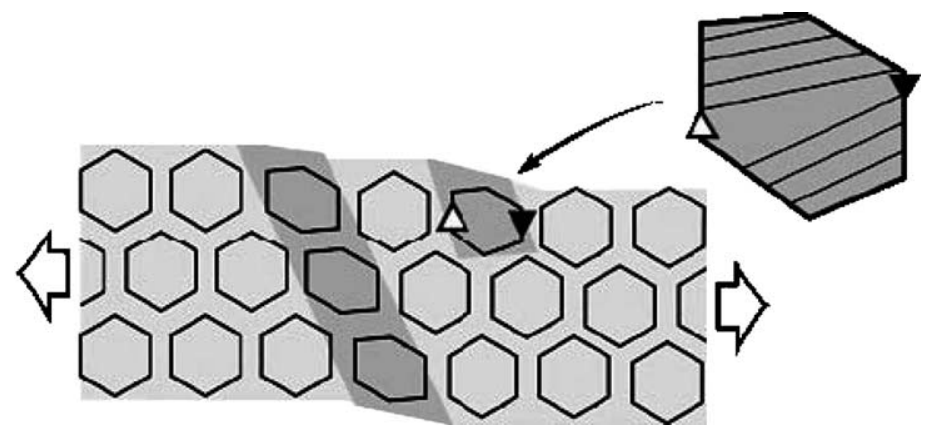

Fig. 39. Rotational deformation in nanaostructures occurs via motion of dipoles of disclinations (triangles), causing crystal lattice rotation behind them (reprinted from [313] with permission from the American Association for the Advancement of Science). 
relationship for superplasticity can be characterized by the following constitutive equation:

$$
\dot{\varepsilon}=A \frac{\mathrm{DGb}}{k T}\left(\frac{b}{d}\right)^{p}\left(\frac{\sigma}{G}\right)^{n}
$$

where $\dot{\varepsilon}$ is the strain rate, $D$ is the adequate diffusion coefficient, $G$ is the shear modulus, $b$ is the Burgers vector, $k$ is the Boltzmann constant, $d$ is the grain size, $T$ is absolute temperature, $p$ is the grain size exponent, $\sigma$ is the applied stress and $n$ is the stress exponent. The grain size exponent $(p)$ has a value between 2 and 3, depending upon whether lattice diffusion or grain-boundary diffusion is the controlling mode of deformation. Eq. (E21) implies that a reduction in grain size can lead to an increase in the superplastic strain rate at constant temperature, or to a reduction in the superplastic temperature at constant strain rate [319,320]. Superplasticity in microcrystalline materials is attained in low strain rates from $10^{-5}$ to $10^{-3} \mathrm{~s}^{-1}$. Such a strain rate range is rather low for industrial forming of structural materials. There is considerable interest in the search of materials with high-strain-rate superplasticity (HSRS) having strain rates $\geq 10^{-2} \mathrm{~s}^{-1}$. HSRS is generally observed at relatively high temperatures of $\sim 0.8 T_{\mathrm{m}}$ (melting temperature) in microcrystalline metals [321-323]. HSRS behavior at lower temperatures is of technological importance owing to its cost effectiveness of near-net-shape forming operations.

Nanocrystalline metals have been found to exhibit superplasticity at lower homologous temperatures (ratio of the test temperature to the melting point) and at higher strain rates than their microcrystalline counterparts. Superplasticity of nanocrystalline metals and alloys follows the general trend of the constitutive relation (Eq. (E21)) but with important differences in the level of stress and strain hardening rates [319]. Lu and coworkers reported that electrodeposited nanocrystalline copper exhibits an elongation higher than 5000\% upon rolling at room temperature [324]. However, a large ductility in rolling or compression is not a real indicator of superplasticity because neck instability and cavitation are absent during such deformation. In this aspect, it is necessary to characterize the tensile superplasticity for nanocrystalline materials at low temperatures and at high strain rates. Experimental observations of tensile superplasticity of nanocrystalline materials at room temperature are very scarce. Tensile superplasticity has been observed in nanocrystalline $\mathrm{Pb}-62 \mathrm{Sn}$ alloy at room temperature possibly due to its low melting point [325]. Low temperature and high strain rate tensile superplasticity has been found to occur in nanocrystalline $\mathrm{Ni}, \mathrm{Ni}_{3} \mathrm{Al}$ and aluminum alloy $(1420 \mathrm{Al}$; $\mathrm{Al}-$ $\mathrm{Mg}-\mathrm{Li}$ ) at 350,300 and $725^{\circ} \mathrm{C}$, respectively. The Ni sample is prepared via electrodeposition whereas $\mathrm{Ni}_{3} \mathrm{Al}$ and 1420 aluminum alloy are fabricated by a severe plastic deformation process. The grain sizes for $\mathrm{Ni}, \mathrm{Ni}_{3} \mathrm{Al}$ and $1420 \mathrm{Al}$ alloy are 20,50 and $100 \mathrm{~nm}$, respectively [319,326-328]. Stress-enhanced grain growth is observed in these materials, particularly for $\mathrm{Ni}$ at $350{ }^{\circ} \mathrm{C}$. The transition from low plasticity to superplasticity in Ni coincided with the onset of grain growth, and may be explained by a combination of the activation of grain-boundary sliding and increased diffusion. For intermetallic $\mathrm{Ni}_{3} \mathrm{Al}$, grain growth is inhibited by the kinetic barrier of preferred atomic pairing between the species present [326]. Fig. 40 is a macrograph showing tensile superplasticity in some nanocrystalline materials. Among them, $1420 \mathrm{Al}$ alloy is of particular interest because of its higher superplastic strain rate $\left(1 \times 10^{-2} \mathrm{~s}^{-1}\right)$ and lower superplastic temperature $\left(300^{\circ} \mathrm{C}\right)$. Fig. 41 shows typical stress-strain curves of microcrystalline and nanocrystalline $\mathrm{Ni}_{3} \mathrm{Al}$ [319]. It is apparent from this figure that the nanocrystalline $\mathrm{Ni}_{3} \mathrm{Al}$ alloy exhibits superplactic ductility at $725^{\circ} \mathrm{C}$ and at a higher strain rate of $10^{-3} \mathrm{~s}^{-1}$ compared to the microcrystalline counterpart at $1050{ }^{\circ} \mathrm{C}$ with a strain rate of $6 \times 10^{-4} \mathrm{~s}^{-1}$. Moreover, high superplastic flow stress and strain hardening are observed in nanocrystalline $\mathrm{Ni}_{3} \mathrm{Al}$. In contrast, microcrystalline alloy exhibits very low flow stress. The origin of high flow stresses in nanocrystalline metals/alloys is still unclear, possibly associated with grain boundary sliding and 


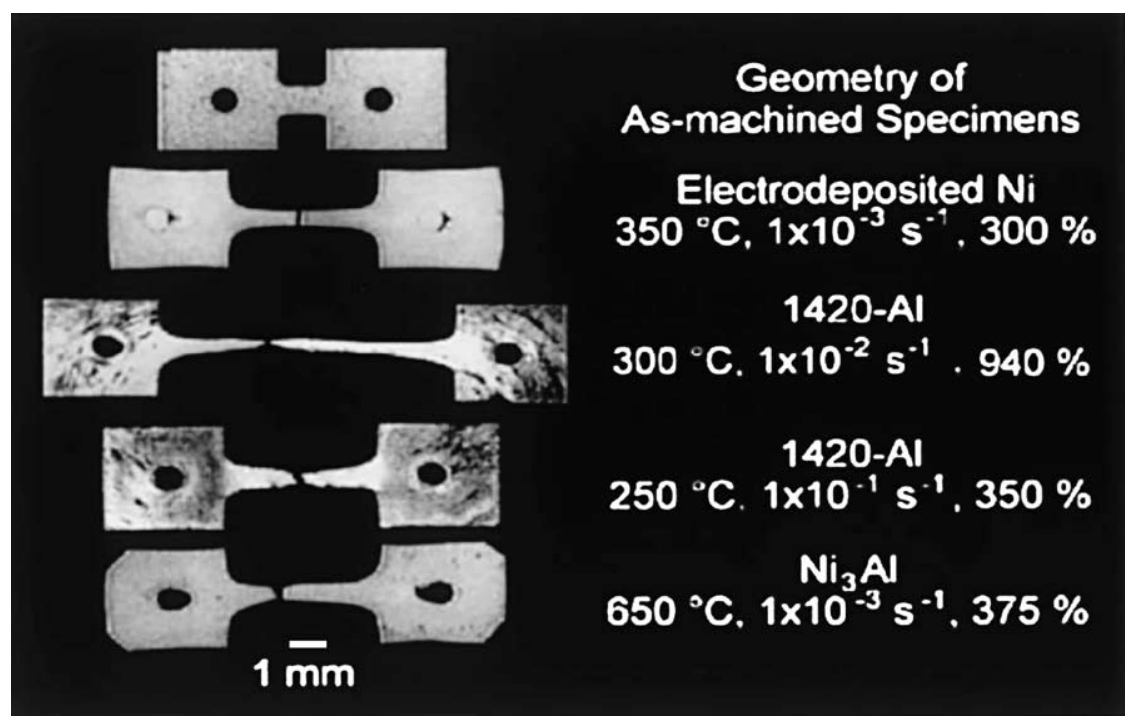

Fig. 40. Tensile superplasticity in nanocrystalline $\mathrm{Ni}, 1420 \mathrm{Al}$ alloy and $\mathrm{Ni}_{3} \mathrm{Al}$ (reprinted from [319] with permission from Elsevier).

diffusion. Superplastic processes also occur in nanoceramics at lower temperatures and at high strain rates as compared to the coarse-grained ceramics [329-332]. For example, nanocrystalline titania $\left(\mathrm{TiO}_{2}\right)$ deforms at $250-300{ }^{\circ} \mathrm{C}$ lower temperatures than micro-grained titania, when equivalent stressstrain rate conditions are imposed [330-332].

In addition to grain boundary sliding, diffusion along grain boundaries [333-335] and along triple junctions [336-340] has been proposed to account for the plastic deformation without dislocation motion below the critical grain size. Diffusion is particularly relavant to the deformation of nanocrystalline metals because typical dislocation mechanisms are suppressed and grain-boundary-controlled deformation processes become dominant [308]. Masumura et al. proposed an analytical model in which $\mathrm{H}-\mathrm{P}$ strengthening prevails at larger grains whilst Coble creep with a threshold stress dominates for smaller grains. In other words, the transition from $\mathrm{H}-\mathrm{P}$ strengthening to Coble creep occurs when the grain size reaches a critical value [341]. The creep rate $(\mathrm{d} \varepsilon / \mathrm{d} t)$ due to grain boundary

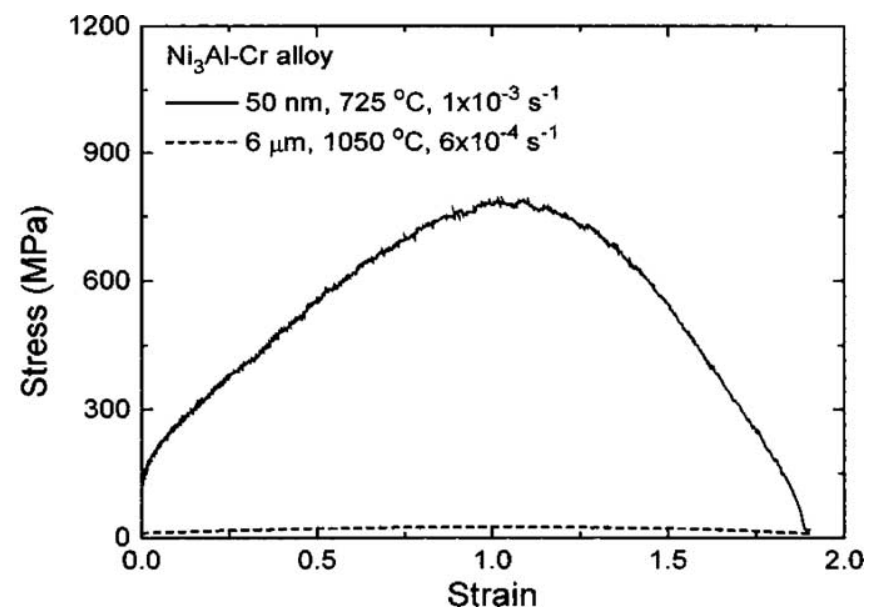

Fig. 41. Flow behavior of microcrystalline and nanocrystalline $\mathrm{Ni}_{3} \mathrm{Al}$ (reprinted from [319] with permission from Elsevier). 
diffusion (Coble creep) is given by [342]:

$$
\dot{\varepsilon}=A^{\prime} \frac{\sigma \Omega_{\mathrm{D}}}{k T} \frac{\delta_{\mathrm{D}} D_{\mathrm{GB}}}{d^{3}}
$$

where $\delta_{\mathrm{D}}$ is the effective diffusional width of the grain boundary, $\Omega_{\mathrm{D}}$ is the activation or atomic volume, $\sigma \Omega_{\mathrm{D}}$ is the work performed by the stress during an elementary diffusion jump in the grain boundary and $D_{\mathrm{GB}}$ is the grain-boundary diffusion coefficient and $A^{\prime}$ is a constant. Gleiter and coworkers reported that the room temperature diffusivities of nanocrytalline $\mathrm{Cu}$ are about 10-20 orders of magnitude higher than conventional microcrystalline $\mathrm{Cu}$ [343]. Chokshi et al. attributed the inverse $\mathrm{H}-\mathrm{P}$ effect in nanocrystalline $\mathrm{Cu}$ to rapid diffusion creep (Coble creep) at room temperature [286]. However, the inverse cube dependence of the strain rate on the grain size could not be verified experimentally at room temperature. Nieman et al. and Sanders et al. reported that the creep rate of $\mathrm{Cu}$ is two to four orders of magnitude slower than the values predicted by the equation for Coble creep. The samples show logarithmic creep typical of the behavior of conventional materials at room temperature [282,329]. Recently, $\mathrm{Lu}$ and coworkers have performed tensile creep tests on electrodeposited nanograined $\mathrm{Cu}(30 \mathrm{~nm})$ in the temperature range $20-50{ }^{\circ} \mathrm{C}$ [334]. The creep rates of nanograined $\mathrm{Cu}$ are of the same order of magnitude as those calculated from Coble creep. The activation energy for creep is determined to be $0.72 \mathrm{eV}$, which is close to that of grain boundary diffusion in nanocrystalline $\mathrm{Cu}$. Such creep behavior is termed 'interface controlled diffusional creep'. The discrepancy in the creep results of Lu's group with those of Nieman et al. and Sanders et al. is attributed to the difference in the processing conditions of the samples. The samples of Lu's group were prepared by the electrodeposition process whereas consolidated IGC $\mathrm{Cu}$ samples containing internal nanopores were used by Nieman et al. and Sanders et al. Such internal pores would affect the creep behavior of $\mathrm{Cu}$.

For nanocrystalline Ni, Wang et al. [335] reported that grain-boundary diffusion and sliding play an important role in room temperature creep deformation of electrodeposited $\mathrm{Ni}(6-40 \mathrm{~nm})$. At a small grain size of $6 \mathrm{~nm}$, the stress exponent $(n)$ derived from the strain rate-stress relation $\left(\mathrm{d} \varepsilon / \mathrm{d} t \sim \sigma^{n}\right)$ is $\sim 1.18$, lying between that of Coble creep $(n=1)$ and grain-boundary diffusion-controlled grainboundary sliding $(n=2)$. At grain sizes between 20 and $40 \mathrm{~nm}$, higher values of $n$ up to 5.3 result, indicating a transition to dislocation creep with increasing grain sizes. Very recently, Yin et al. [344] studied the creep deformation of electrodeposited $\mathrm{Ni}$ with $30 \mathrm{~nm}$ grains at room temperature $(290 \mathrm{~K})$ and at $373 \mathrm{~K}$. The stress exponent values for nanocrystalline $\mathrm{Ni}$ at room temperature and $373 \mathrm{~K}$ are determined to be 1.1 and 6.5, respectively. The rate of diffusional Coble creep exhibits a linear dependence on true applied stress. Thus, the deformation of $\mathrm{Ni}$ at room temperature is dominated by a Coble creep mechanism, in which enhanced diffusion along grain boundaries, triple junctions and quadruple nodes control the mass transport process. At $373 \mathrm{~K}$, lattice dislocation gliding and grainboundary sliding may play an important role in the deformation of nanocrystalline $\mathrm{Ni}$.

Swygenhoven and Caro [301] conducted MD simulations for the creep of nanocrystalline Ni samples at temperatures below $120 \mathrm{~K}$. They reported that the mechanisms responsible for creep deformation at low temperature are grain boundary sliding, grain rotation and grain boundary motion. As grain-boundary diffusion creep is one of the mechanisms responsible for the deformation of nanocrystalline materials, Gleiter and coworkers used MD simulations to study the diffusion creep in silicon and palladium at high temperatures [307,308]. By using appropriate interatomic potentials, the microstructure of silicon consists of nanograins having high-angle grain boundaries with grain sizes up to $7.5 \mathrm{~nm}$. The creep behavior is controlled by grain-boundary diffusion and described quantitatively by Coble creep, i.e. strain rate increases with decreasing grain size as $1 / d^{3}$ (Fig. 42). The activation energy for grain-boundary diffusion creep is the same as that for grain-boundary self-diffusion 


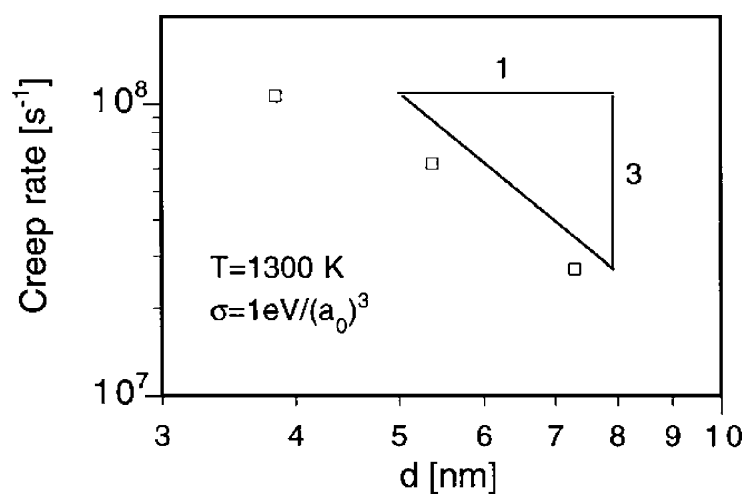

Fig. 42. MD simulation of Coble creep in nanocrystalline Si showing the log-log plot of the creep rate vs. grain size. For the two largest grain sizes studied, the creep rat approaches the $1 / d^{3}$ depenence predicted by the Coble creep (reprinted from [307] with permission from Kluwer Academic Publishers).

(Fig. 43a and b). For nanocrystalline Pd, the activation energy for grain-boundary diffusion creep $(0.61 \mathrm{eV})$ is the same as that for grain-boundary self-diffusion (Fig. 44). When the grain diameter becomes of the order of the grain boundary width, the grain-size dependence of the strain rate changes from $d^{-3}$ to $d^{-2}$ (Fig. 45). This implies that the grain boundary width, $\delta$, is non-negligible compared with the grain size $(d)$ in the nanocrystalline metals. Taking grain boundary width into consideration, the Coble creep rate equation is modified to:

$$
\dot{\varepsilon}=4 K \frac{\sigma \Omega_{\mathrm{D}} D_{\mathrm{GB}}}{k T}\left(\frac{2 \delta}{d^{3}}-\frac{\delta^{2}}{d^{4}}\right)
$$

where $K$ is a material constant. In the limit of large grain size, i.e. $d \geq \delta$, the second term inside the bracket of Eq. (E23) is negligible compared to the first one, yielding the Coble creep as expressed in Eq. (E22). In the limit of small grain size, $d \approx 2 \delta$, the second term is comparable to the first one, and the resulting creep rate becomes

$$
\dot{\varepsilon}=3 K \frac{\sigma \Omega_{\mathrm{D}} D_{\mathrm{GB}}}{k T} \frac{1}{d^{2}}
$$
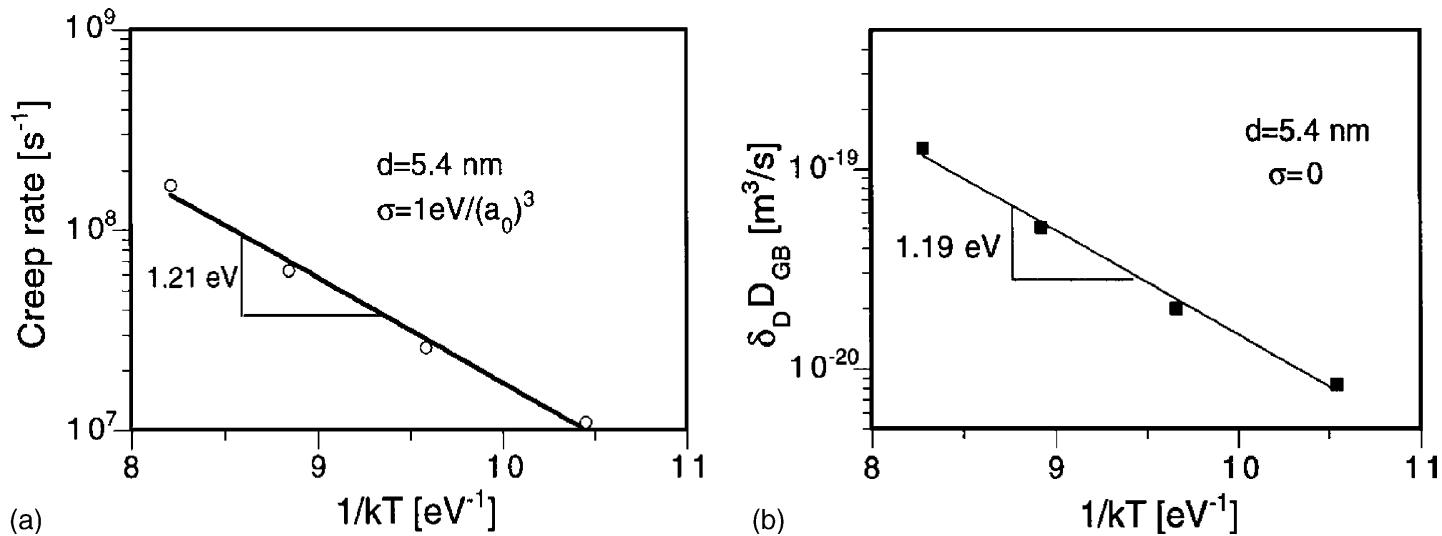

Fig. 43. MD simulation of Coble creep in nanocrystalline Si: (a) Arrhenius plot of the creep rate between 1100 and $1400 \mathrm{~K}$ and (b) activation energy for grain-boundary self-diffusion under zero stress (reprinted from [307] with permission from Kluwer Academic Publishers). 


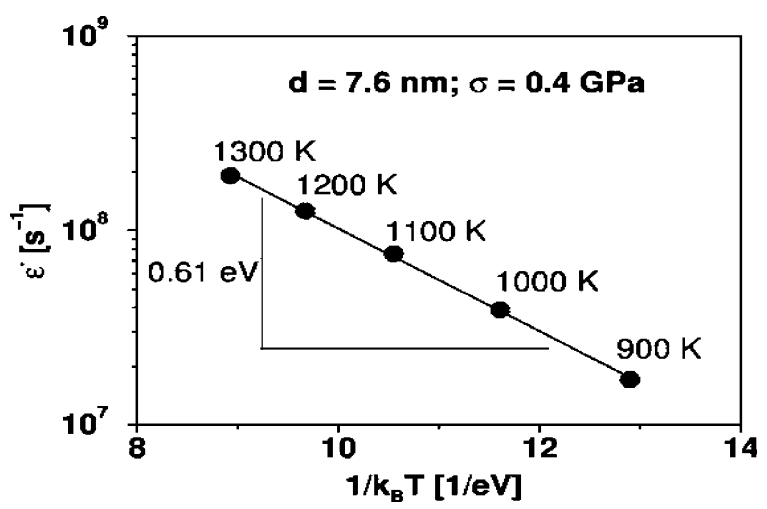

Fig. 44. MD simulation in nanocrystalline Pd showing Arrhenius plot of the creep rate between 900 and $1300 \mathrm{~K}$ (reprinted from [308] with permission from Elsevier).

It is evident that the grain size dependence of grain boundary diffusion creep becomes identical to the Nabarro-Herring creep model $\left(\dot{\varepsilon} \propto\left(1 / d^{2}\right)\right)$ when the grain boundary width becomes comparable to the grain size. Therefore, Eq. (E23) extends the validity of the Coble creep equation from coarse-grained polycrystals to nanocrystalline materials, provided that the creep mechanism remains under the control of grain-boundary diffusion process. This implies that grain-boundary diffusion and grainboundary sliding form an integral process of creep deformation. Grain boundary sliding is needed to accommodate the homogeneous grain elongation takes place induced by Coble creep, with the entire deformation process being controlled by grain-boundary diffusion [308]. These results disagree with the MD prediction of Swygenhoven and Caro [301] in which the grain boundary sliding, without diffusion accommodation, is proposed as the principal room-temperature deformation mechanisms in fcc nanocrystalline $\mathrm{Ni}$.

In most cases, creep diffusion is enhanced along the grain boundaries and triple junctions. With decreasing grain size in nanocrystalline materials to $10 \mathrm{~nm}$ or lower, the effects of triple junctions on the deformation become significant because the volume fraction of triple junctions is very high. The volume fraction of triple junction approaches that of grain boundaries when the grain size is decreased

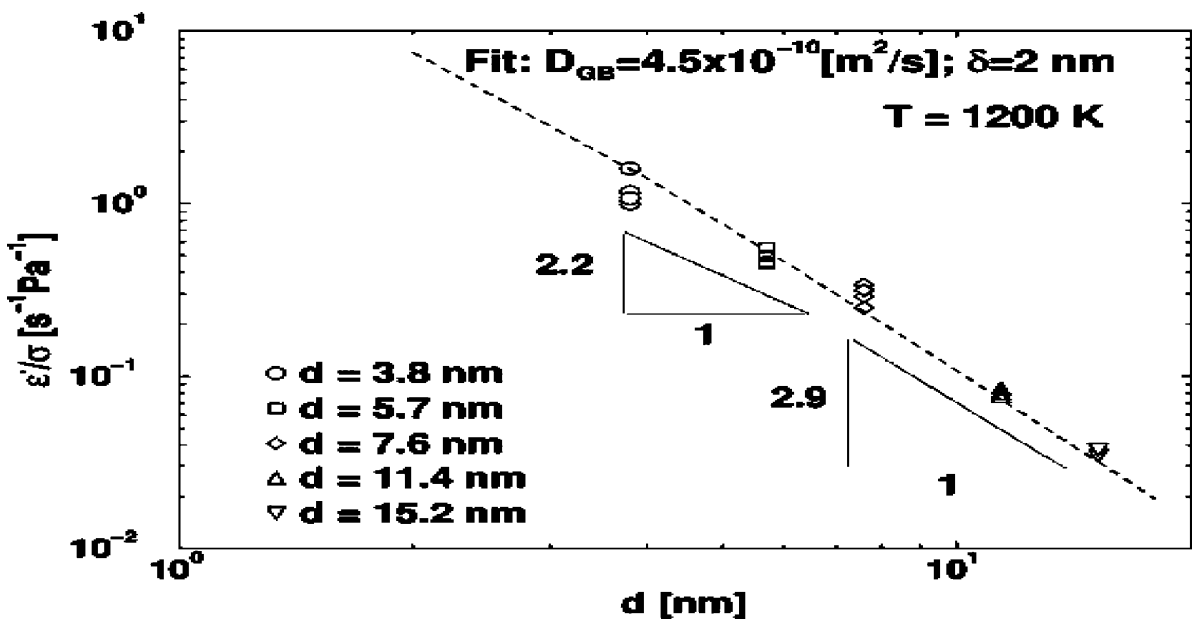

Fig. 45. MD simulation in nanocrystalline Pd showing $\log -\log$ plot of $\dot{\varepsilon} / \sigma$ with at $1200 \mathrm{~K}$. The dash line shows a fit with Eq (E23) with estimated values of the fitted parameters $D_{\mathrm{GB}}=4.5 \times 10^{-10} \mathrm{~m}^{2} / \mathrm{s}$ and $\delta=2 \mathrm{~nm}$ (reprinted from [308] with permission from Elsevier). 
to $\sim 3 \mathrm{~nm}$. In this respect, softening of the $\mathrm{H}-\mathrm{P}$ relation occurs when the volume fraction of triple junctions in the microstructure reaches a significant value [290]. It has been found that triple junctions of grain boundaries play the role of enhanced diffusivity pipes, nuclei of second phase segregation, sources of lattice dislocations during plastic deformation, drag centers of grain boundary migration upon grain growth, nucleation site for voids, etc. [289,336-340,344-346]. For example, triple junctions have been reported to enhance the Coble creep diffusion of electrodeposited $\mathrm{Ni}$ with $30 \mathrm{~nm}$ grains [344]. Grain-boundary triple junctions could act as softening regions during deformation in sharp contrast to grain boundaries, which are well-known hardeners. The triple junctions play the role of softeners possibly due to their low density in which diffusion transport is fast $[339,345]$. However, the exact role of triple junctions in plastic deformation of nanocrystalline materials is not clearly understood.

In general, consolidated nanocrystalline samples are too small to be used in conventional, largescale mechanical tests. In this regard, indentation method appears to be an effective route to study the creep deformation of nanocrystalline materials. Indentation creep, in which the extent of deformation is measured during both loading and unloading, has been studied in nanocrystalline materials by monitoring the changes in the indentation size occurring beneath the indenter as a function of time. In the process, the indenter is pushed into the surface at a fixed rate of indentation until a predetermined load or penetration depth is reached, then the load is held fixed while the indenter continues to creep into the material. Li and coworkers [347,348] have demonstrated the use of indentation and impression creep in investigating the creep deformation mechanism of materials at high homologous temperatures. In other words, identation creep technique can be used to determine the power law stress exponent and activation energy. The results obtained from indentation creep translate readily into those determined from tensile loading. At low temperature, Stone and coworkers reported that the activation volume, $V^{*}$, can be measured using indentation method [349-353]. $V^{*}$ is defined as a measure of the Burger's vector (b) times the area swept out by dislocation during the process of thermal activation, the so-called "activation area" or $A^{*}$ [354]. Mathematically, $V^{*}$ from a uniaxial compressive or shear stress is given as

$$
V^{*} \approx \frac{k T}{\partial \tau / \partial \ln \dot{\varepsilon}}
$$

where $\tau$ is the shear stress and $\dot{\varepsilon}$ the strain rate in shear. The term $(\partial \tau / \partial \ln \varepsilon)$ is called the rate sensitivity of the flow stress in shear. On the basis of the indendation hardness, Stone and Mustafa determined the $V^{*}$ as

$$
V^{*} \approx \frac{9 k T}{\partial H / \partial \ln \dot{\varepsilon_{\mathrm{eff}}}}
$$

and the strain rate sensitivity, $m$, is given by

$$
m \approx \frac{\partial H}{\partial \ln \dot{\varepsilon_{\mathrm{eff}}}}
$$

where $H$ is the indentation hardness and $\dot{\varepsilon_{\text {eff }}}$ is an effective strain rate in the plastic zone beneath the indenter. Stones and Elmustafa demonstrated that the activation volume data determined from nanoidentation creep for high purity aluminum and alpha brass samples scales with the activation volume measured using conventional uniaxial loading [351].

Despite the fact that nanocrystalline metals exhibite high strength and hardness, they are very brittle with little tensile ductility compared to their coarser-grained counterparts $[355,356]$. Ductility is of utmost importance in many forming operations and in the prevention of catastrophic failure during 
load-bearing applications. The tensile elongation of consolidated IGC $\mathrm{Cu}$ and $\mathrm{Pd}$ metals with grain sizes below $50 \mathrm{~nm}$ is quite low, i.e. in the range of 1.6-4\%. In contrast, $\mathrm{Cu}$ with a grain size of $110 \mathrm{~nm}$ exhibits an elongation to failure $>8 \%$ [69]. Compressional ductility in nanocrystaline $\mathrm{Cu}$ samples is found to be higher than the tensile ductility, with strains of $12-18 \%$ prior to failure, but still much less than the coarse grain form [68]. The low ductility of these nanocrystalline metals resulted from inferior sample quality due to the presence of microvoids and impurities [69]. To diminish artefacts associated with extrinsic processing effects on the mechanical properties, Lu and coworkers prepared a fully dense bulk nanocrystalline $\mathrm{Cu}$ sample with an average grain size of $27 \mathrm{~nm}$ by electrodeposition [357]. A tensile ductility with an elongation to fracture of $30 \%$ is achieved, which is comparable to that for the coarse-grained $\mathrm{Cu}$. However, conflicting results are reported for the tensile properties of commercially available electrodeposited nanocrystalline Ni. Dalla Torre et al. [358] reported that the plastic strain of commercial electrodeposited Ni samples with a mean grain size of $\sim 21 \mathrm{~nm}$ was very low and less than $4 \%$. They attributed this to the presence of impurities and porosities in the electrodeposited nanocrystalline Ni. Two batches of electroplated Ni having the same mean grain size but different microstructures and impurities contents were used. Metallic impurities and sulfur contaminant originating from the saccharin inhibitor were detected in these two batches. Nanopores could possibly originate from hydrogen being incorporated during electrodeposition. Nearly full density of $99.5 \pm 0.5 \%$ was achieved despite the presence of hydrogen-filled nanopores.

Even for the case in which the porosities of nanocrystalline $\mathrm{Cu}$ are removed via surface mechanical attrition treatment (SMAT), the tensile ductility remains low $(\sim 2 \%)$, with fracture occurring very soon after yielding [359]. The SMAT process involves shot peening of the surface layer to induce plastic deformation without changing the chemical composition in a short period of time. The surface layer is free of porosity and contamination accordingly. Fig. 46 shows typical stressstain curves for the SMAT prepared nanocrystalline $\mathrm{Cu}$, IGC powder consolidated nanocrystalline $\mathrm{Cu}$ [343] and conventional coarse-grained $\mathrm{Cu}$ [359]. Thus, there are factors other than porosity that limit the ductility of nanocrystalline metals. These factors include instability of crack nucleation and growth as well as plastic instability [359,360]. In defect-free nanocrystalline metals, the ability for strain hardening is needed to avoid premature failure. Early plastic instability in nanocrystalline metals is manifested in the form of shear banding, leading to rapid catastrophic failure due to excessive localized deformation. Evidence of shear bands has been seen in nanocrystalline metals like $\mathrm{Cu}, \mathrm{Pd}, \mathrm{Ni}$ and Fe under tension [282,292,358] or compression [361-364]. Fig. 47a shows typical shear bands

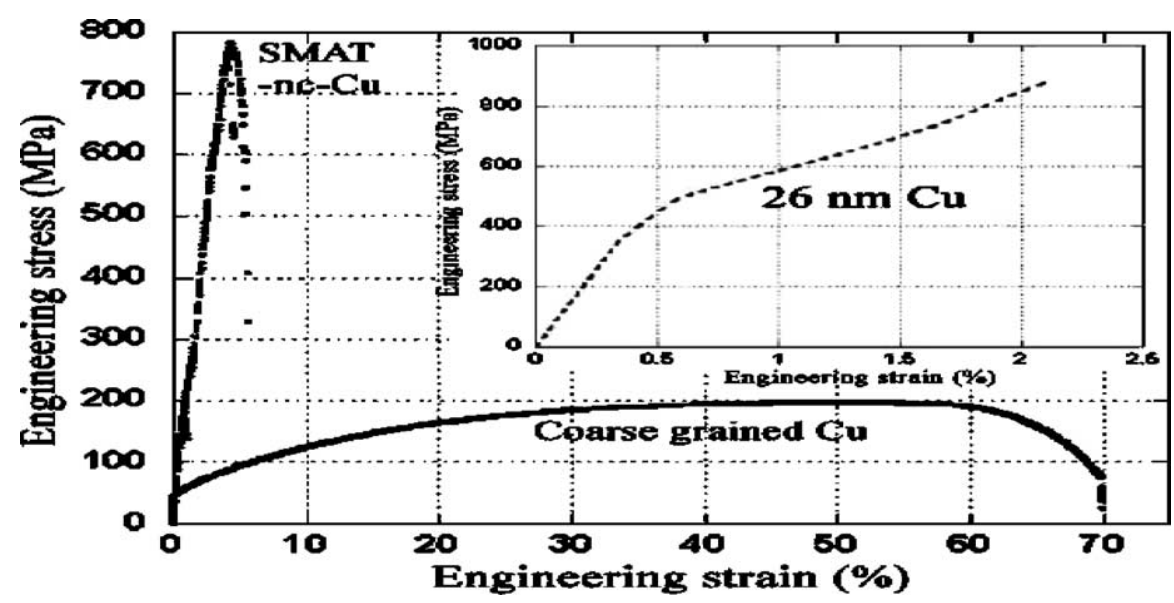

Fig. 46. Engineering stress-strain curves of SMAT nanocrystalline $\mathrm{Cu}$, IGC powders consolidated nanocrystalline $\mathrm{Cu}$ and conventional coarse-grained $\mathrm{Cu}$ specimens (reprinted from [359] with permission from Elsevier). 

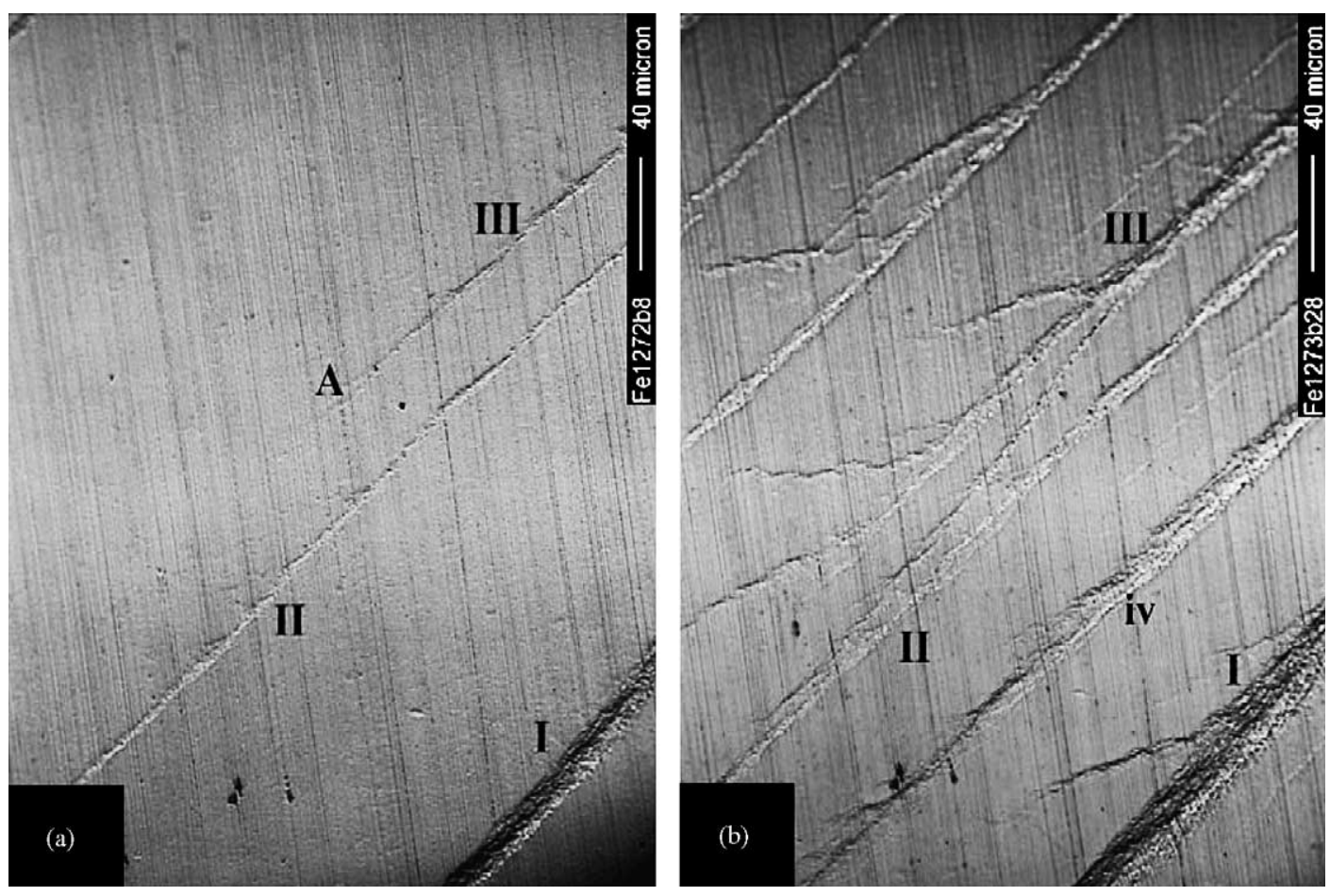

Fig. 47. Formation and development of shear bands in $268 \mathrm{~nm}$ Fe after uniaxial compression to plastic strain levels of: (a) $3.7 \%$ and (b) $7.8 \%$ at (1-4) $\times 10^{-4} \mathrm{~s}^{-1}$. Loading axis is vertical (reprinted from [363] with permission from Elsevier).

developed in Fe with grain sizes $\leq 268 \mathrm{~nm}$ at a plastic strain of $3.7 \%$ under uniaxial compression. With increasing the plastic strain to $7.8 \%$, broadening of existing shear bands (bands I, II and III) and propagation of bands (band III) into several branches are observed. New shear bands (IV) are also nucleated at this stage. The development of a network of shear bands at about $\pm 45^{\circ}$ to the loading axis at $7.8 \%$ plastic strain is summarized in Fig. 47b. TEM observations reveal that grains in the band are elongated along the shear direction. In contrast, equiaxed grains are observed outside the band (Fig. $48 \mathrm{a}$ and $\mathrm{b}$ ).

Mechanical properties of nanocrystalline materials are also found to be strain-rate dependence. Enhanced strain rate sensitivity at room temperature with decreasing grain size is observed in nanoceramics, such as $\mathrm{TiO}_{2}$ and $\mathrm{ZnO}[365,366]$. Lu et al. [367] studied the effect of strain rates $(6 \times$ $10^{-5}$ to $1.8 \times 10^{3} \mathrm{~s}^{-1}$ ) on the tensile flow behavior of electrodeposited nanocrystalline $\mathrm{Cu}$ (Fig. 49). They reported that the flow stress (with $1 \%$ plastic strain) increases slowly from 84 to $122 \mathrm{MPa}$ with increasing strain rate. However, the strain to failure and fracture stress are found to increase dramatically with increasing strain rates, especially at a high strain rate of $1.8 \times 10^{3} \mathrm{~s}^{-1}$. In contrast, the fracture strain of conventional coarse-grained $\mathrm{Cu}$ decreases slightly at high strain rates [367]. The tensile properties of electrodeposited nanocrystalline $\mathrm{Ni}$ have been investigated in considerable detail $[335,358,368]$. Dalla Torre reported that the average tensile strength of Ni samples is $\sim 1388 \pm$ $61 \mathrm{MPa}$ from $5.5 \times 10^{-5}$ to $5.5 \times 10^{-2}$, but it increased dramatically to $2500 \mathrm{MPa}$ at a strain rate of $10^{3} \mathrm{~s}^{-1}$. The tensile ductility appears to decrease dramatically with increasing strain rates from strain rates of $5.5 \times 10^{-5}$ to $5.5 \times 10^{-2} \mathrm{~s}^{-1}$ [358]. Such a decreasing trend contradicts with the data of Lu et al. [367] in which the tensile ductility increases significantly with the strain rates. More recently, Schwaiger at al. employed both tensile and depth-sensing indentation techniques to examine the fracture behavior and damage evolution of electrodeposited nanocrystalline Ni $(20 \mathrm{~nm})$, ultrafine 

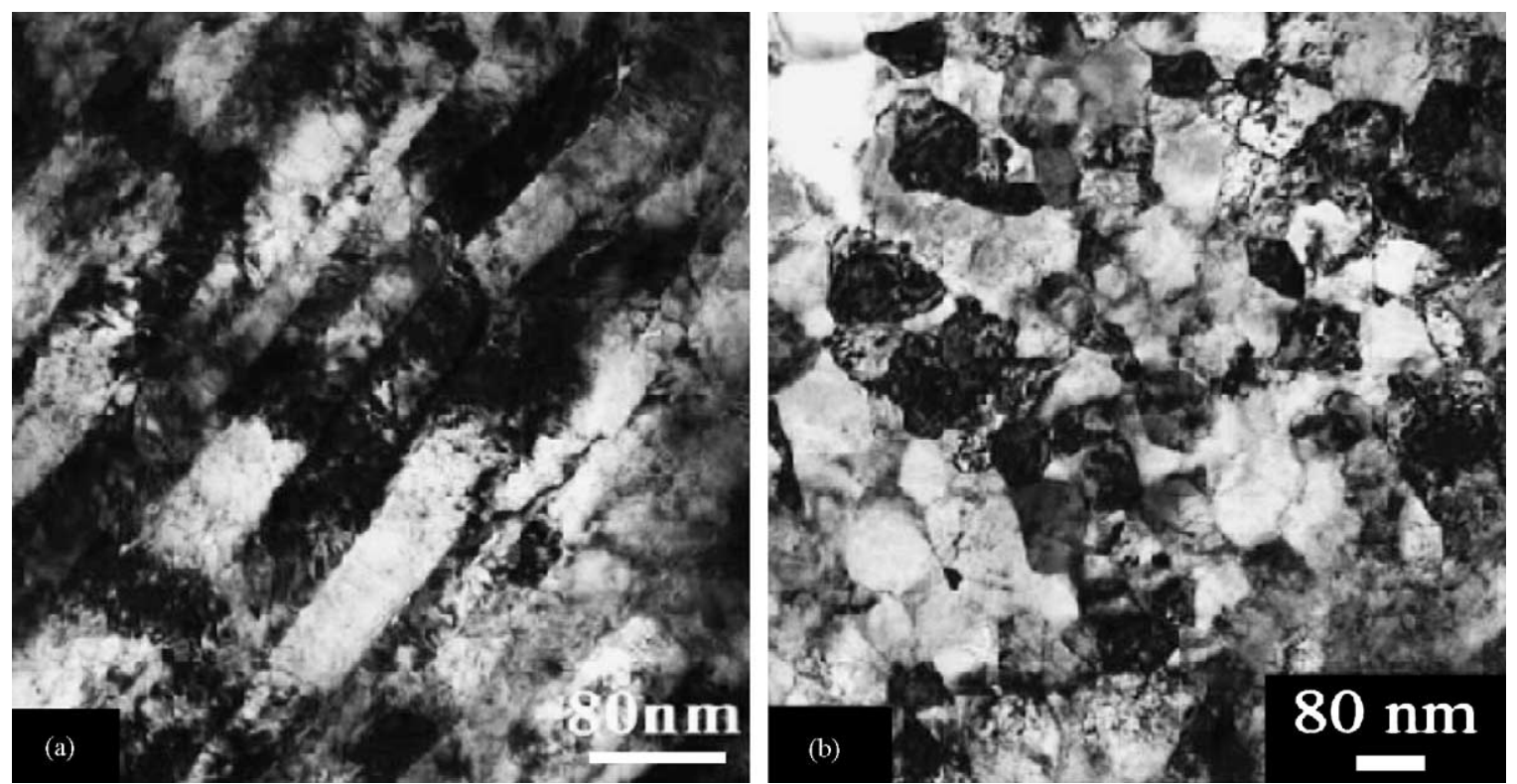

Fig. 48. TEM micrographs taken (a) within and (b) outside a shear band in $136 \mathrm{~nm}$-Fe under uniaxial compression (reprinted from [363] with permission from Elsevier).

grained Ni (100-1000 $\mathrm{nm})$ and microcrystalline Ni $(>1 \mu \mathrm{m})$ specimens [368]. From the results of both measurements, they indicated that the flow stress of nanocrystalline Ni increases with increasing stain rate. This effect was not observed in ultrafine grained and microcrystalline $\mathrm{Ni}$ specimens. The positive strain rate sensitivity in the flow stress of nanocrystalline $\mathrm{Ni}$ was attributed to the localized plastic deformation in grain boundaries and the nearby regions. They termed such regions as grainboundary affected zones (GBAZ). Due to the lack of dislocation sources in nanocrystalline Ni, the grain interior deformed at stress levels close to the theoretical strength, and contributed little to overall

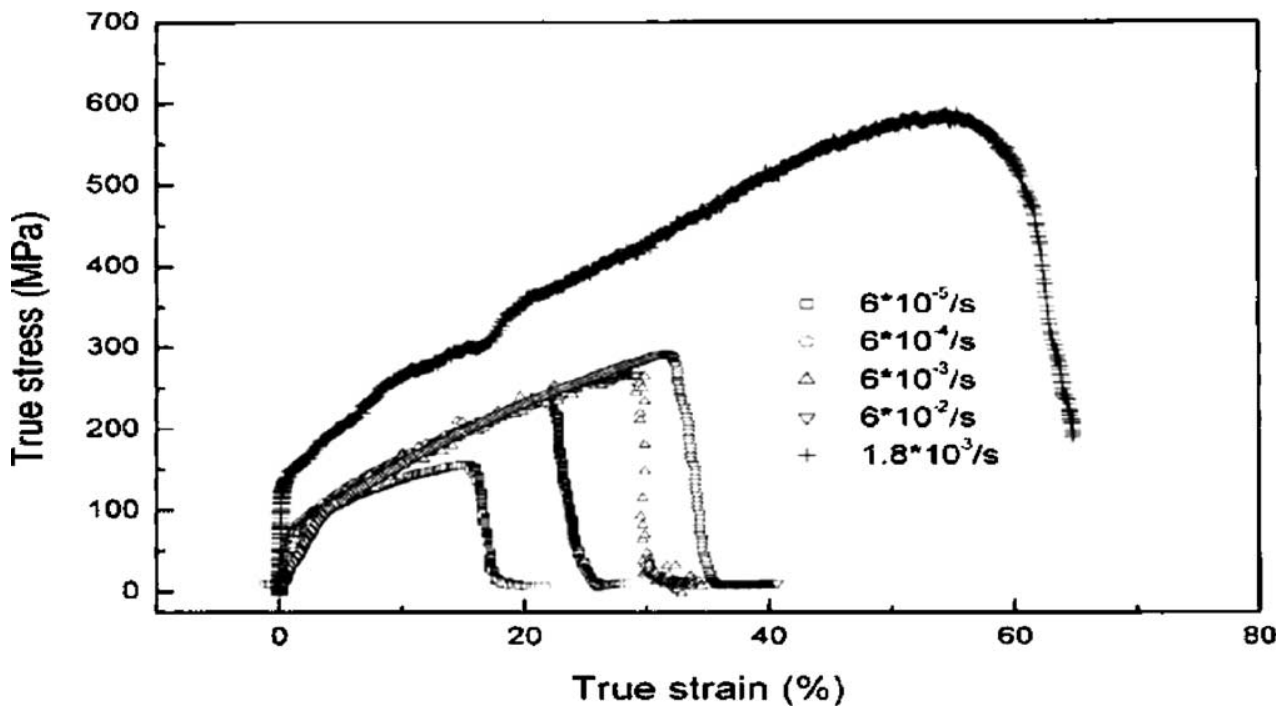

Fig. 49. True stress-strain curves for electrodeposited nano-Cu obtained at room temperature under various strain rates (reprinted from [367] with permission from Elsevier). 
plastic deformation. The whole nanocrystalline sample was made up of plastically softer GBAZ and plastically harder grain interiors. Thus, the observed rate-sensitivity aroused from the GBAZ [368]. This approach is based on the MD simulation of Schiotz et al.in which the grain boundaries are elastically softer than the grain interiors of nanocrystalline metals. In this case, most of the deformation occurs in the grain boundaries. Also the strain-rate dependence in flow stress is observed for the simulated stress-strain curves of nano- $\mathrm{Cu}$ [305].

Basic understanding of structure-property relationships is essential to elucidate the mechanisms responsible for the deformation of nanocrystalline metals. The technique of in situ straining of TEM foil specimens is effective to explore the deformation and grain-boundary structures of nanocrystalline materials [317,346,369]. TEM observation is focused within localized small areas of samples, hence the results may not be representative of the bulk behavior. Ke et al. conducted in situ deformation on nanocrystalline gold films with grain diameters of $\sim 10 \mathrm{~nm}$ [317]. They found, by observing relative fringe rotation in different grains, that deformation occurred by grain-boundary sliding and grain rotation. No evidence of dislocation activity was detected during straining. Youngdahl et al. [369] strained nanocrystalline IGC $\mathrm{Cu}$ foils in a TEM, and videotaped the experiments. The grain size distribution of the $\mathrm{Cu}$ sample was very broad, ranging from 2 to $500 \mathrm{~nm}$, with the majority lying between 50 and $80 \mathrm{~nm}$. Dislocation activity-induced deformation was observed in grains with diameters of $\sim 30-100 \mathrm{~nm}$. There was no direct evidence for grain-boundary sliding or rotation. The presence of dislocation activity and absence of the grain boundary sliding or rotation in strained $\mathrm{Cu}$ foils are derived from their larger grain sizes. This result is in sharp contrast to the observation of $\mathrm{Ke}$ at al. mentioned above. Legros et al. [355] designed a microsample tensile testing device to strain dog-bone-shaped microsample tensile specimens ( $3 \mathrm{~mm}$ in overall length with a $200 \mu \mathrm{m} \times 200 \mu \mathrm{m}$ cross-sectional gauge). Subsequent TEM examination found no dislocation activity in nanocrystalline $\mathrm{Ni}$ with an average grain size of $28 \mathrm{~nm}$. However, SEM observation revealed extensive dislocationbased plasticity in $\mathrm{Al}$ with a large grain size, i.e. $250 \mathrm{~nm}$. In a recent in-situ strained TEM study, Kumar et al. [346] observed copious dislocation activity in the region around the crack tip of electrodeposited $\mathrm{Ni}$ with nanograins of $\sim 30 \mathrm{~nm}$. Voids were formed at grain boundaries and triple junctions in the regions ahead of the crack tip. Further, ex-situ SEM examination revealed the presence of dimpled failure in nanocrystalline $\mathrm{Ni}$. The size of dimples was six to ten times larger than the average grain size of Ni. From TEM and SEM observations, they concluded that grain-boundary controlled deformation played a key role in fcc nanocrystalline metals with grain sizes below $30 \mathrm{~nm}$. Above a critical grain size of $30 \mathrm{~nm}$, dislocation-based plasticity was dominant. This is inconclusive evidence for the occurrence of a critical grain size in which the H-P relation deviates from the normal positive slope.

The information on the mechanical behavior of nanocrystalline ceramics is relatively fewer compared to that of nanocrystalline metals. In the past few years, research on nanocrystalline ceramics has beeen driven by the interest to improve sinterability, increase ductility, and reduce temperature for superplasctic deformation for near net shape forming. As long as the nanocrystalline ceramics can retain their finer grain sizes after sintering, they can exhibit better mechanical properties than their coarser-grained samples. At room temperature, nanocrystalline ceramics may have a small amount of ductility beyond that exhibited by large-grained ceramics, but they are not superplastic. At high temperatures, nanocrystalline ceramics exhibit significant compressive ductility and strain rate sensitivities that are indicative of superplasticity [370]. Siegel et al. [371] reported that nanophase $\mathrm{TiO}_{2}$ (rutile) exhibits considerable improvement in both the sinterability and resulting mechanical properties relative to coarser-grained samples. The nanophase $\mathrm{TiO}_{2}$ (rutile) is observed to sinter at 400-600 ${ }^{\circ} \mathrm{C}$ lower temperatures than the coarser-grained counterparts and without the need for compacting and sintering aid, i.e. polyvinyl alcohol. In contrast, coarser-grained $\mathrm{TiO}_{2}$ samples do not sinter effectively without sintering aid. 
Gleiter and coworkers were the first to report the occurrence of superplasticity in $\mathrm{TiO}_{2}$ film during compressive loading [372]. Grain size refinement in ceramics usually results in flow stress reduction and improved ductility at high temperatures. The maximum grain size for superplasticity in ceramics is about $1 \mu \mathrm{m}$, which is nearly on tenth of that in metals. For superplastic metals, the value of stress exponent in Eq. (E21) is usually well defined and reproducible between different sets of experiments. There are significant differences in the values of $\mathrm{n}$ and $\mathrm{p}$ reported in the literature for nominally identical materials tested under similar testing condtions. These discrepancies can be rationalized in terms of a threshold stress, $\sigma_{0}$. The effective stress driving the deformation is not the applied stress but rather $\left(\sigma-\sigma_{0}\right)$ [373]. Strain rate sensitivity defined as the slope of logarithmic plot of the flow stress vs. strain rate. It is an inverse of stress exponent $(n)$ and an important parameter in superplasticity. However, the $m$ values reported in the literature for ceramics show a large scatter, i.e. they could vary from 0.3 to 1 . For example, Nieh and Washword reported than $m$ of tetragonal zirconia poly crystal (TZP) stabilized by $3 \mathrm{~mol} \%$ yttria, i.e. 3Y-TZP is about 0.3 [374], but Wakai et al. reported $\mathrm{m}$ is about 0.5 [375], or others even higher [376]. Langdon attributed this to the presence of a wide range of impurity levels in ceramics [377]. It is worthnoting that fined-grained Y-TZP of $0.3 \mu \mathrm{m}$ exhibits extensive tensile elongation of $800 \%$ at $1550{ }^{\circ} \mathrm{C}$ under a strain trate of $8.3 \times 10^{-5} \mathrm{~s}^{-1}$ [374], and $1038 \%$ in $\mathrm{SiO}_{2}$-doped TZP at $1400{ }^{\circ} \mathrm{C}$ under a strain rate of $1.3 \times 10^{-4} \mathrm{~s}^{-1}$ [378]. This is attributed to the stability of fine grain size in TZP in comparison with other ceramics such as $\mathrm{Al}_{2} \mathrm{O}_{3}$. The grain sizes are stabilized in TZP materials by the presence of second phases [379]. Grain boundary sliding is the primary mechanism responsible for deformation in superplastic ceramics [373,379].

Nanocrystalline ceramics exhibit superplasticity at faster strain rates and lower temperatures than their coarser-grained counterparts [370,380]. Grain size refinement in 3Y-TZP to nanoscale can lead to a factor of 34 increase in strain rate at $1150{ }^{\circ} \mathrm{C}$ (Fig. 50). The effects of grain size and temperature on the stress-strain rate relation of $\mathrm{TiO}_{2}$ are shown in Fig. 51. It can be seen that nanocrystalline $\mathrm{TiO}_{2}$ achieves the same strain rates and stresses as coarser-grained rutile, but at $250-300{ }^{\circ} \mathrm{C}$ lower temperatures. Similarly, Chen et al. indicated that the superplastic forming temperature (1120 $1250{ }^{\circ} \mathrm{C}$ ) of Y-TZP of $100 \mathrm{~nm}$ at a given strain rate is lowered by $200{ }^{\circ} \mathrm{C}$ in comparison with the standard Y-TZP materials [381]. Hahn and Averback [382] have performed compressive creep test in HIP consolidated nanocrysalline $\mathrm{TiO}_{2}$ dense compacts at $600-810{ }^{\circ} \mathrm{C}$. The compresion creep results were analyzed using an empirical equation modified from Eq. (E21). The stress exponent was of the order of $2.2-3$ for nanocrystalline $\mathrm{TiO}_{2}$ at $600-810{ }^{\circ} \mathrm{C}$. In contrast, room temperature nanoidentation

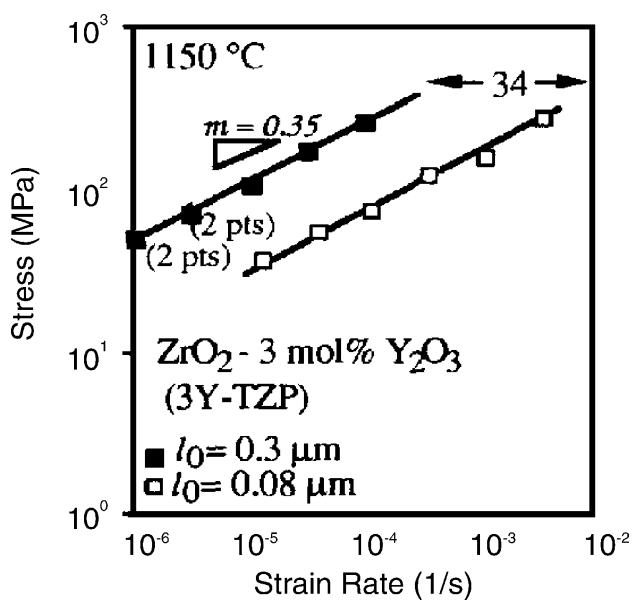

Fig. 50. Log-log plot of stress vs. strain rate for $3 \mathrm{Y}-\mathrm{TZP}$ at $1150{ }^{\circ} \mathrm{C}$ (reprinted from [380] with permission from Elsevier). 


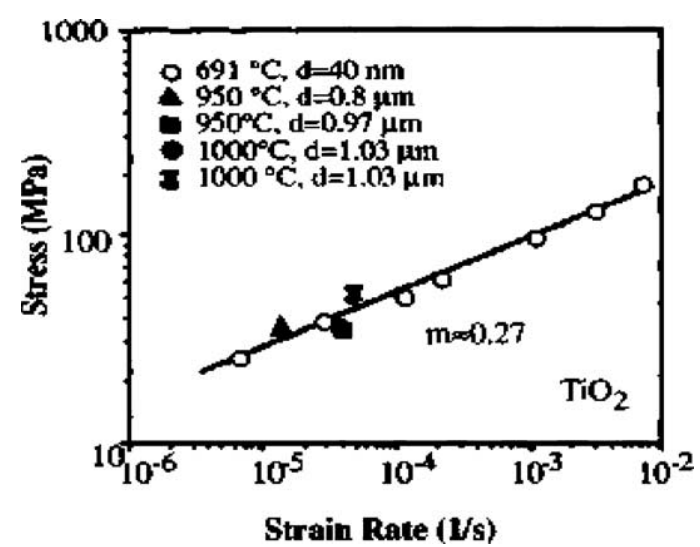

Fig. 51. Log-log plot of stress vs. strain rate for nanocrystalline and micro-grained $\mathrm{TiO}_{2}$ (reprinted from [380] with permission from Elsevier).

test reveals that the strain rate sensitivitiy values of nanoceramics are extremely small, i.e. an order of magnitude below the value of $m=1 / 3$ needed for superplasticity (Fig. 52). This implies the absence of superplasticity $(m=\sim 0.3)$ or Coble creep $(m=1)$ in nanoceramics at room temperature. However, room temperature creep and superplasticity are reported to occur in nanocrystalline $\mathrm{Cu}$ and electrodeposited $\mathrm{Ni}$ as mentioned previously.

Structural materials are often subjected to fatigue failure during their service lives. The resistance to fatigue failure is one of the critical design requirements for structural materials subject to cyclic loadings. Information concerning the fatigue behavior of nanocrystalline and submicron metals is rather scarce. Recently, Hanlon et al. [383] studied the high cycle fatigue (HCF) behavior of submicron and nanocrystaline Ni under stress-controlled conditions. They reported that both submicron and nanocrystaline $\mathrm{Ni}$ samples have a higher endurance limit than conventional coarsergrained Ni. It is generally known that the HCF performance of materials is highly dependent on their mechanical strength. The submicron and nanocrystaline Ni samples exhibit higher tensile strength

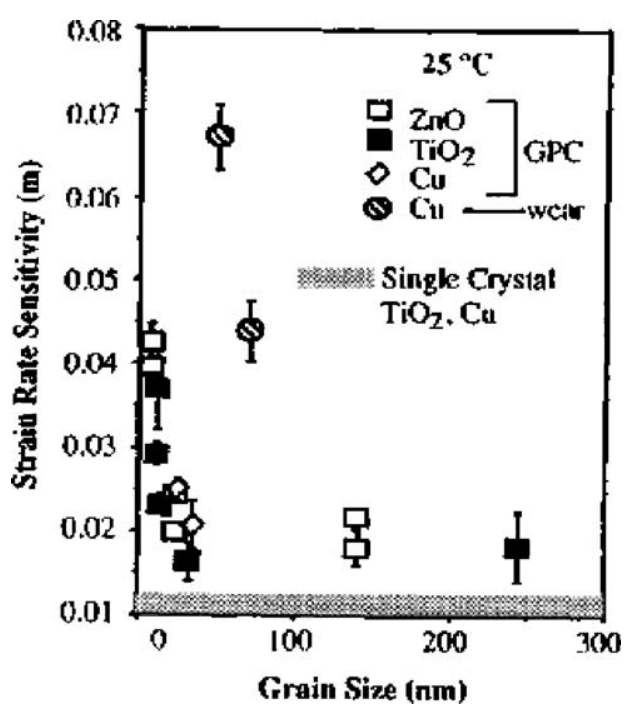

Fig. 52. Strain rate sensitivity $(m)$ as a function of grain size for $\mathrm{TiO}_{2}$ and $\mathrm{ZnO}$ nanoceramics prepared by gas phase condensation (GPC). Data for nanocrystalline GPC $\mathrm{Cu}$ and wear debris $\mathrm{Cu}$ are also shown for comparison purposes. (reprinted from [380] with permission from Elsevier). 
than coarser polycrystalline $\mathrm{Ni}$, thereby possessing a higher endurance limit. In contrast, low cycle fatigue (LCF) life of the structural materials under strain-controlled loadings is determined mainly by their tensile ductility. It is expected that nanocrystalline metals and their alloys exhibit poorer strain controlled properties than their coarser polycrystalline counterparts. Very little work has been devoted to the LCF and HCF deformation characteristics of submicron Al-alloys produced by equal channel angular pressing [384,385]. A remarkably large enhancement in fatigue life is observed in submicron Al-alloy compared to coarser-grained counterparts subjected to stress-controlled cycling [385]. However, deterioration in LCF response has been found with grain refinement [384].

In summary, there is a need for understanding the fundamentals of the strengthening of nanocrystalline materials. Many attempts have been made to interpret the origin of the observed deviations from the H-P relation. However, the explanations for inverse H-P effect remain controversial. These include the presence of nanopores, grain-boundary sliding, grain-boundary rotation, contribution of triple junctions and Coble creep diffusion. The mechanism by which the softening takes place is poorly understood at present. This is attributed to limited or availability of appropriate experimental tools or routes to measure the mechanical responses of nanocrystalline materials below the critical grain size. The inability to prepare pore- and contaminant-free material with a similar microstructure within a single processing method is one of the big challenges we face. Nanocrystalline metals generally exhibit limited tensile plasticity, but possess large plastic strains under compression and rolling. Nanocrystalline $\mathrm{Cu}$ has been reported to experience large plastic strains during rolling at room temperature and to undergo creep at or near room temperature [324,334]. In contrast, room temperature superplasticity is absent in nanoceramics partly due to their strain rate sensitivity $(m)$ values are very small, i.e. an order of magnitude below the value of $m=1 / 3$ needed for superplasticity to occur.

\section{Nanocrystalline coatings}

Coating is a surface modification technique to the base material in order to enhance the physical and/or mechanical performances of tools and structural components. Existing PVD and CVD processes for preparing the microcrystalline coatings can be used to produce nanostructred coatings by modifying the processing parameters or by using feedstock powders having nanograined structures. The availability of various nanoparticles prepared from vapor, liquid and solid routes have motivated the development of nanocrystalline coatings with superior wear and oxidation resistances as well. Conventional plasma spraying and high velocity oxygen fuel thermal spraying are viable high-rate deposition techniques to produce nanocrystalline coatings using nanosized feedstock powders.

In the past decade, hard ceramic coatings have attracted increasing attention due to their potential applications in cutting and forming tools, bearings, engine parts, seals, etc. Hard coatings improve the durability of substrate materials in hostile environments against abrasive wear, thus prolong the tool life. The coating materials for wear protection can be classified according to the chemical bonding character: metallic, covalent or ionic hard materials. Metallic hard materials are borides, carbides and nitrides of the transition metals (Fig. 53) [386]. Under various available PVD processes, ion beam assisted deposition (IBAD) is particularly effective to fabricate transition metal nitride nanocrystalline coatings with good adhesion and controlled microstructures. IBAD has the advantage of having more independent processing parameters than other PVD methods. For examples, the energy and flux of bombarding ions can modify the size and crystallographic orientation of grains. The ever-growing need for superior materials to withstand severe operating conditions has driven the search for superhard coatings with hardness values above $40 \mathrm{GPa}$. Significant research has been conducted 


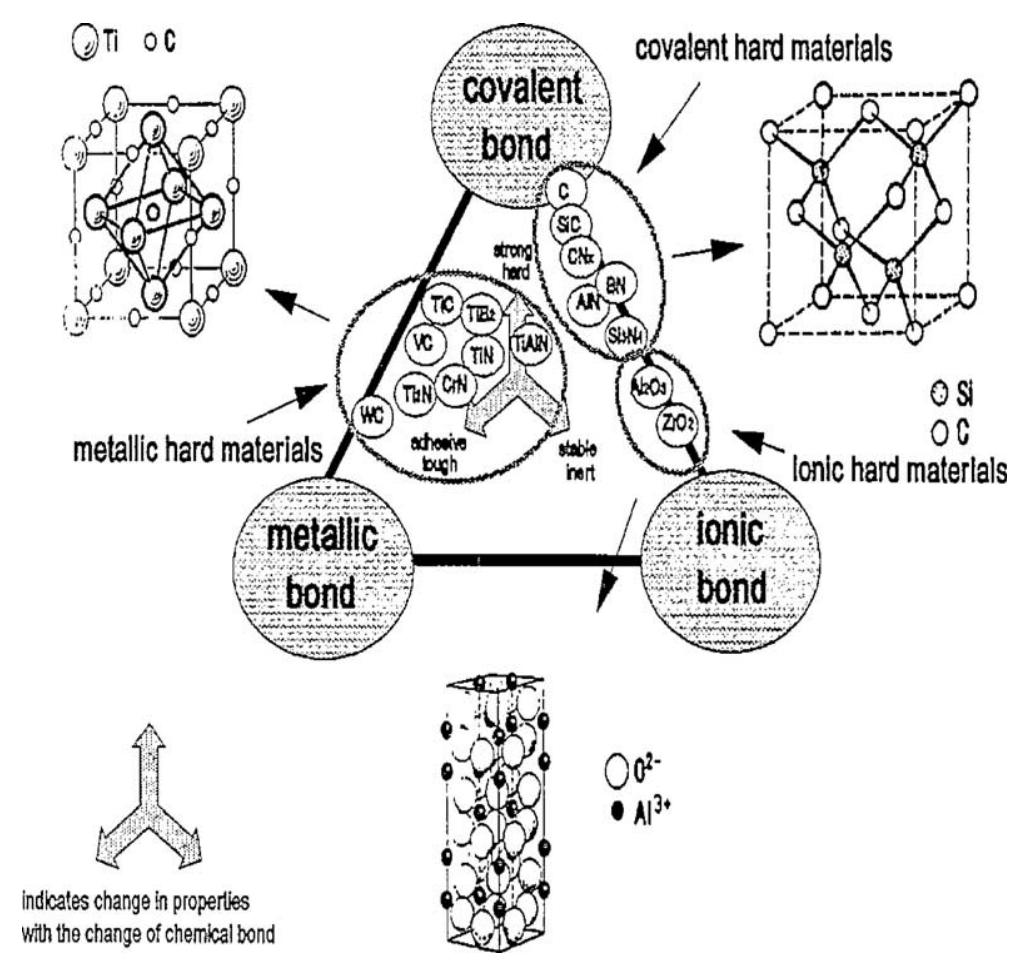

Fig. 53. Different groups of hard materials with characteristic crystal structure (reprinted from [386] with permission from Elsevier).

recently on the design of superhard coatings with excellent strength, hardness and toughness characteristics. One of the design concepts is based on the formation of mutilayered structures or superlattices having a difference in the elastic modulus between the layers. The thickness of each layer should be in the nanometer range such that no dislocation source could operate within the layers. By tailoring the thicknesses of the individual layers, it is possible to produce multilayered films with desired nanohardness values. Another design approach utilizes the formation of single layer nanocomposite coatings with microstructures comprising of crystallites, with grain sizes in the nanometer level, embedded in an amorphous matrix. Softening of the coatings due to an inverse HallPetch effect can be avoided by blocking the grain boundary sliding.

\subsection{Thermal sprayed coatings}

Thermal spraying is a well-established technology for forming hard coatings on selected component substrates. The coating material is heated in a gaseous medium and projected at high velocity as molten droplets onto a substrate surface. Upon impact, the droplets become flattened, transfer the heat to the cold substrate and solidify rapidly to form the 'splats'. Powders, rod and wires can be used as feedstock materials. Several processing routes can be employed depending on the materials and desired coating performances. These include plasma spraying, high velocity oxyfuel (HVOF) spraying, detonation flame spraying, flame spraying, etc. In conventional plasma spraying, a high temperature plasma jet is developed inside the gun. Powder particles of several micrometers are injected into a plasma jet, causing them to melt into droplets that are propelled towards the substrate. The HVOF uses a combustion process to rapidly heat and accelerate the powder coating material to high velocities. Combustion fuel gas such as acetylene, propane, propylene or hydrogen is premixed 

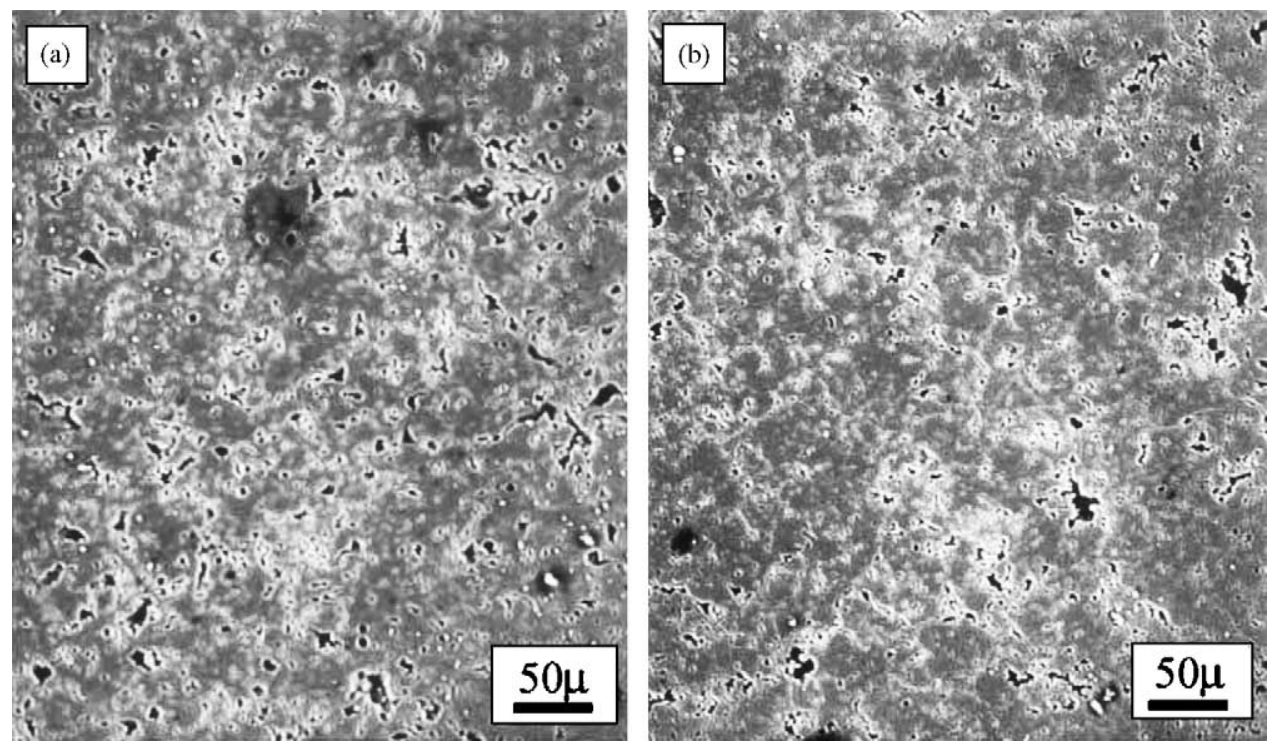

Fig. 54. SEM micrographs showing surface morphologies of air plasma-sprayed zirconia coatings deposited from (a) microcrystalline and (b) nanocrystalline powders (reprinted from [396] with permission from Elsevier).

with oxygen. The gaseous mixture is then ignited, causing a hypersonic flame with velocity of approximately $2000 \mathrm{~m} / \mathrm{s}$. Powder particles are introduced into the combustion chamber by an inert gas carrier such as argon. They are heated and accelerated under a hypersonic flow towards the substrate. High-quality microcrystalline metallic and ceramic coatings can be fabricated by low-pressure plasma spraying and HVOF spraying [387-392]. The availability of a variety of nanopowders, including IGC, aerosol processing, sol-gel processing, solution chemical synthesis and mechanical alloying/milling has attracted increasing interest in the development of nanocrystalline coatings in the past few years. Thermal spraying methods using nanopowders have yielded coatings with higher hardness, strength and wear resistance than those of the conventional counterparts. The nanoscale microstructure can be retained in the coatings despite the high temperatures and melting involved in forming a dense coating. Plasma spraying [393-399] and HVOF spraying [400-403] of nanostructured feedstock powders of metals or ceramics proved to be potential methods to form nanocrystalline coatings. The HVOF method is preferred to plasma spraying because of its higher droplet velocity and lower thermal energy levels, yielding denser structure, and higher bond strength between the coating and the substrate.

Oxide ceramics such as alumina, chromia, titania and zirconia have been used widely as surface coating materials to improve wear, erosion, cavitation and corrosion resistances. Zirconia-based coatings have been applied as thermal barrier coatings for piston crowns and cylinder heads in internal combustion engines to improve thermal efficiency, fuel economy and power output [404]. Fig. 54a and $\mathrm{b}$ are SEM micrographs showing the surface morphologies of air plasma-sprayed zirconia coatings of $600 \mu \mathrm{m}$ prepared from microcrystalline $(1-5 \mu \mathrm{m})$ and nanoparticle $(70-110 \mathrm{~nm})$ powders, respectively [396]. Both coatings contain some pores, which are typical characteristics of the plasma-sprayed coatings. Nanocrystalline zirconia coatings exhibit lower porosity $(8 \%)$ than that of microcrystalline coatings $(12 \%)$. TEM examination of the microstructure of the nanocrystalline coating reveals the presence of equiaxed grains of 60-120 nm, and columnar grains with lengths of 150-350 nm (Fig. 55a and $b$ ). The fined equiaxed grains are resulted from homogeneous nucleation of a highly undercooled melt whilst columnar grain growth arised from heterogeneous nucleation at splat boundaries where a higher gradient of cooling rate exists. Due to the good melting of nanostructured zirconia feedstock in 

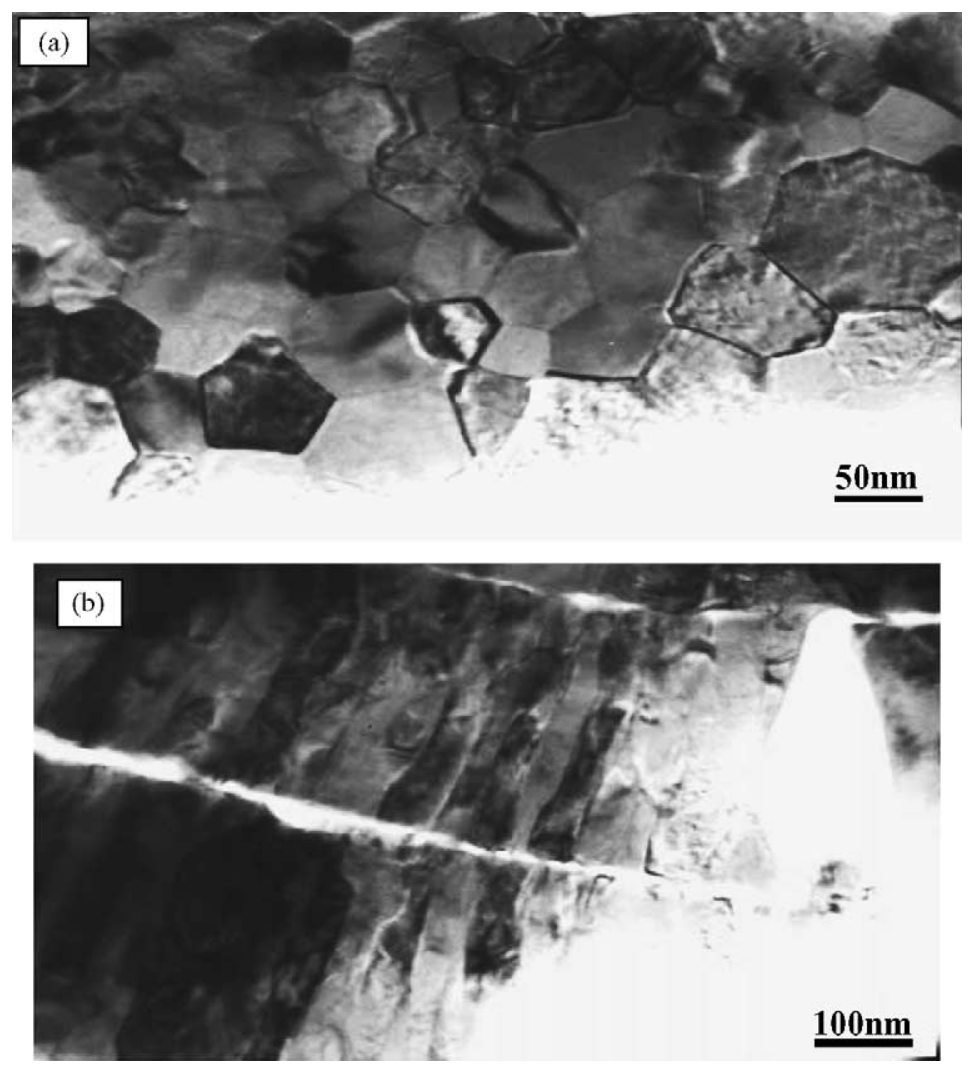

Fig. 55. TEM micrographs showing (a) equiaxed grains and (b) columnar grains developed in air plasma-sprayed nanocrystalline zirconia coating on stainless steel substrate (reprinted from [396] with permission from Elsevier).

the plasma jet, the single splat is very thin and the interfaces between two splats are narrowed. This leads to an improvement of adhesion between the coating and the substrate. Accordingly, nanostructured zirconia coatings with lower porosity exhibit a lower friction coefficient and wear rate compared to their microcrystalline counterparts (Fig. 56).

\subsection{Transition metal nitride coatings}

The use of hard coatings for the protection of structural components against wear is widely recognized. Transition nitride coatings like TiN, TiCN, TiBN and TiAlN are well-suited for increasing the performance of cutting tools and drills due to their high hardness $[405,406]$. Transition metal nitrides are also important materials in the decorative coating industry because they exhibit prominent coloration in the visible wavelength region [407]. Titanium nitride hard coatings deposited by PVD and CVD have been implemented on an industrial scale for many years. For practical purposes, nitride hard coatings must be firmly adhered to the substrates [408-411]. However, PVD is a line-of-sight process, and the adhesion of the coating to the substrate is somewhat lower that that for CVD. This is because diffusion of the coating material can take place into the substrate during thermal CVD process. The main limitation of thermal CVD is the corrosive nature of precursor gases, e.g. $\mathrm{SiH}_{4}$, $\mathrm{SiCl}_{4}$ or $\mathrm{TiCl}_{4}$ gases which are used in the process may pose problems of health and fire hazards. Moreover, the substrates may experience surface distortion upon exposure to the high temperature environment. For a variety of applications, e.g. bearing balls, drills and gears, a low deposition 


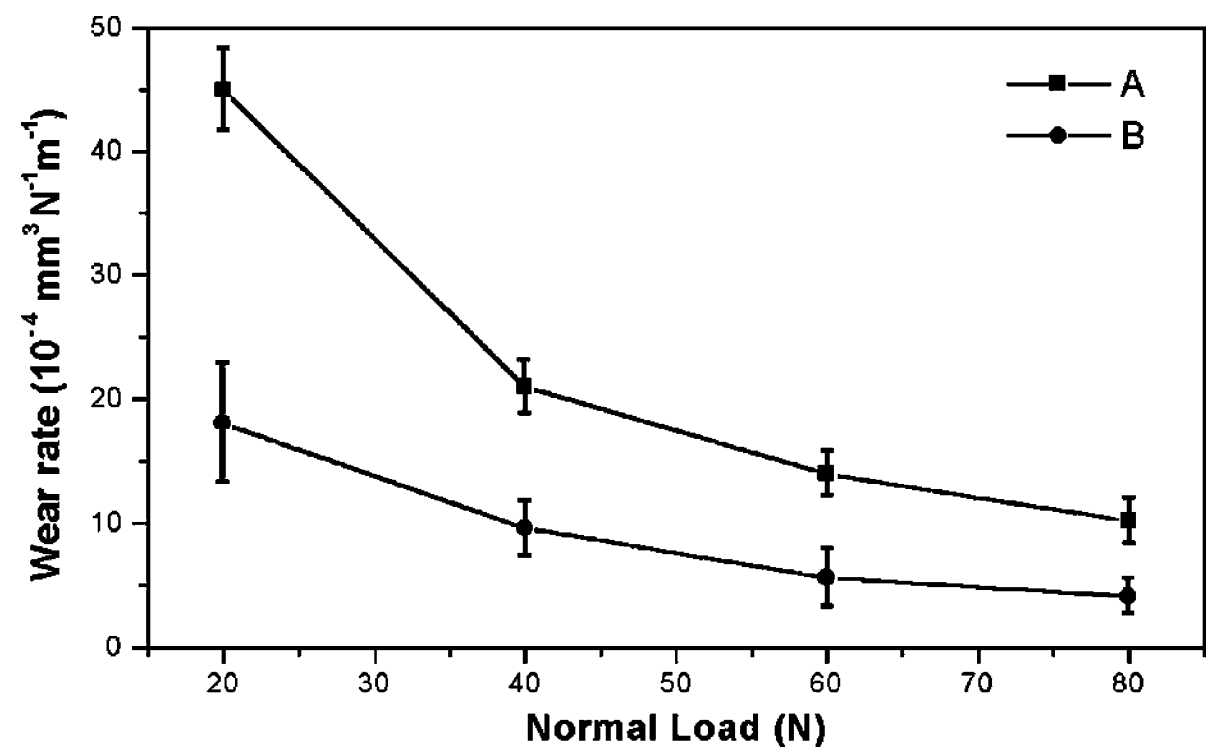

Fig. 56. Variation of wear rate with the applied load for the air plasma-sprayed zirconia coatings: (A) microcrystalline coating; (B) nanostructured coating (reprinted from [396] with permission from Elsevier).

temperature is needed to avoid distortion of coated components and loss of mechanical properties. This is difficult to realize in thermal CVD. Though plasma CVD can offer lower deposition temperatures for producing TiN coatings in the range of $480-560{ }^{\circ} \mathrm{C}$, this technology cannot be easily scaled up for industrial use. Only a few commercial and industrial plants have been developed globally [409].

Considerable effort is devoted to preparing nanocrystalline coatings with improved coating adhesion using the ion-assisted PVD methods. Ion bombardment of the growing film can retard the grain growth and allow the formation of nanocrystalline films. IBAD shows great promise for forming metal nitride coatings because significant improvement in wear, corrosion, electrical resistivity and optical properties can be achieved by changes in deposition parameters such as atom flux, ion energy, ion incidence angle, substrate temperature, etc. IBAD refers to the process in which films produced by PVD (evaporation or sputtering) are simultaneously bombarded by an independent ion beam [412414]. IBAD allows the ion flux and energy to be precisely controlled. The motivation for using the IBAD process is the need for independent control of the film composition and better adhesion of the film-substrate system. Significant performance improvements in coating properties have been achieved by changes in deposition parameters such as atom flux, ion energy, ion incidence angle, substrate temperature, etc.

The development of hard coatings based on transition metal nitrides using IBAD is a subject of intensive research. These nitrides include TiN, $\mathrm{CrN}, \mathrm{VN}$ and $\mathrm{ZrN}$ [415-426]; they have distinct chemical and physical properties. As an example, TiN oxidizes steadily at temperatures above $500{ }^{\circ} \mathrm{C}$, leading to the formation of poorly $\mathrm{TiO}_{2}$ adherent rutile phase oxide layer on top of the TiN. The wear protection of TiN coatings is deteriorated accordingly $[427,428]$. $\mathrm{CrN}$ is more resistant to oxidation than TiN owing to the formation of a dense and passive oxide layer of $\mathrm{Cr}_{2} \mathrm{O}_{3}$ that restricts further oxidation [429]. For the most part, IBAD has been utilized in fundamental scientific research rather than in the mass production sectors. In thin film applications, a low energy $(\leq 100 \mathrm{eV})$ ion flux is commonly used to control the microstructure and microchemistries of the films at lower temperatures. The process of low-energy ion bombardment controls the growth of the film by the energy delivered to 
the growing film by bombarding ions [430-432]. Ion bombardment has strong effects on the chemical and structural features of thin films. For TiN thin films prepared by PVD, the (lllll) plane is the most commonly observed preferred orientation. It has been reported that TiN film with (lllll) preferred orientation possess the highest hardness $[433,434]$. However, the TiN preferred orientation can change from $\left(\begin{array}{lll}1 & 1 & 1\end{array}\right)$ to completely $\left(\begin{array}{lll}2 & 0 & 0\end{array}\right)$ by controlling the ion/metal flux $\left(J_{\mathrm{i}} / J_{\mathrm{Ti}}\right)$ ratios $[435,436]$.

Fig. 57 shows a schematic diagram of the IBAD system for forming nanocrystalline TiN thin films. The system consists of an e-beam evaporation source for evaporating Ti metal and an ion source for providing nitrogen ion beams to form TiN on Si. The (2 000$)$ is the dominant preferred orientation in the TiN thin films as the substrate temperature increases [415]. Chen and coworkers employed such system to control the microstructures of $\mathrm{TiN}, \mathrm{VN}$ and $\mathrm{CrN}$ nanocrystalline thin films under bombardment of nitrogen ions of low energies $(150-500 \mathrm{eV})[415,424,426]$. The resulting microstructure of nitrides is interpreted in terms of the Thorton-Messier structure-zone diagram $[437,438]$. In dealing with thin film samples, different structure-zone diagrams have been proposed to correlate the microstructure of thin films with deposition parameters. One of the main parameters of the structure-zone diagrams is homologous temperature $\left(T_{\mathrm{S}} / T_{\mathrm{M}}\right)$, defined as the ratio of the substrate deposition temperature $\left(T_{\mathrm{S}}\right.$, in $\left.K\right)$ to the melting point of thin film material $\left(T_{\mathrm{M}}\right)$ [437-439]. The substrate temperature plays a key role in determining the adatom surface mobility and the bulk diffusion rates. Grovenor et al. examined the microstructure of metal films grown by thermal evaporation [439]. They classified the microstructures into four zones according to their homologous

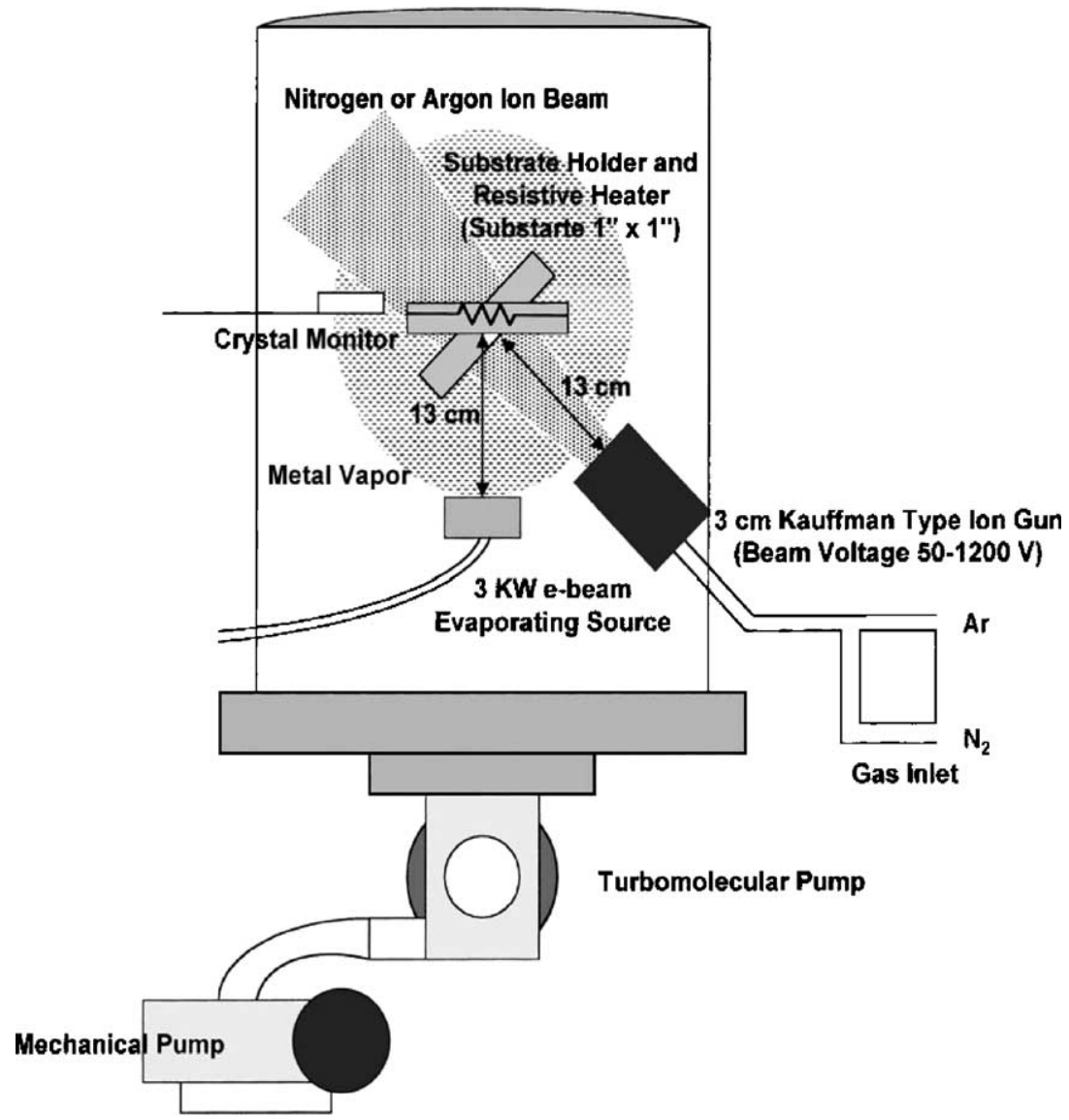

Fig. 57. Schematic diagram of the ion-beam assisted deposition system [415]. 


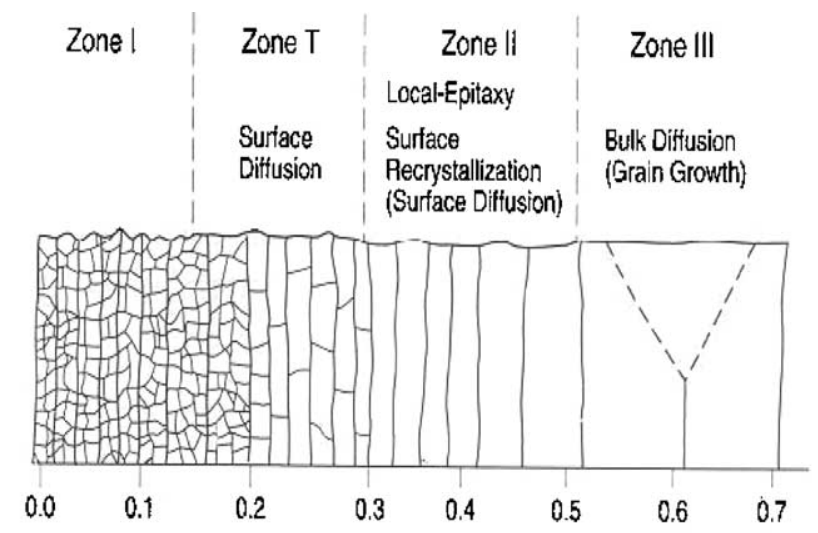

(a) $\quad T_{s} / T_{m}$

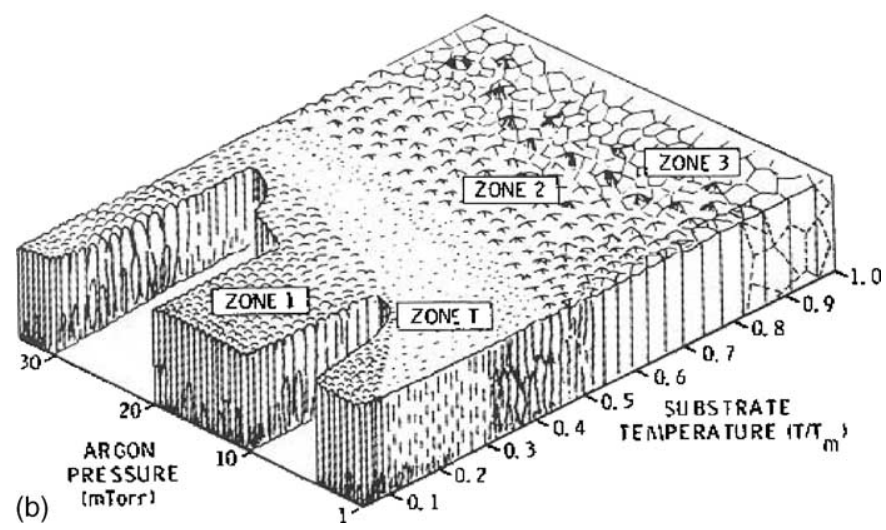

Fig. 58. (a) Grovenor and (b) Thorton structure zone diagrams (reprinted from [437,439] with permission from Elsevier).

temperatures as depicted in Fig. 58a. At substrate temperatures below $0.15 T_{\mathrm{M}}$, the film consists of equiaxed fine grains with grain diameters in the range 5-20 nm (Zone I). The mobility of the deposited atoms is low at lower substrate temperatures. Such equiaxed grains resulted from a high nucleation rate and a low grain growth rate. Zone $\mathrm{T}$ is the transitional microstructure between Zone I and the columnar Zone II, in which the substrate temperatures range from $0.15 T_{\mathrm{M}}$ to $0.3 T_{\mathrm{M}}$. In Zone $\mathrm{T}$, the adatoms can migrate via surface diffusion driven by the higher substrate temperatures. The Zone II structure is columnar, resulting from deposited atoms have sufficient surface mobility to diffuse and to increase the grain size. In Zone III, the grain growth is controlled by bulk diffusion, as the substrate temperatures are higher than $0.5 T_{\mathrm{M}}$ [439]. Thorton [437] incorporated an additional parameter, the sputtering gas pressure in the structure-zone diagram (Fig. 58b). In this diagram, Zone I is the columnar structure with pronounced pores and open columnar boundaries. This structure is promoted by a high working gas pressure or a low homologous temperature. Zone T is located in a higher $T_{\mathrm{S}} / T_{\mathrm{M}}$ or decreasing sputtering gas region, which shows dense arrays of fibrous grains. With increasing the substrate temperatures, surface diffusion of adatoms are enhanced accordingly. A reduction in sputtering gas pressure during deposition increases the mean free path for elastic collisions between coating species and the sputtering gas atoms. This leads to higher kinetic energy of coating species impinging on the substrate surface, thereby producing relatively dense microstructures. The microstructure of Zone II consists of columnar grains separated by distinct boundaries. Zone III is defined as 
that range of conditions where bulk diffusion of adatoms predominates. Considering that the ion beam enhanced the adatom mobility by transferring energy to the adatoms, Messier replaced the sputtering gas pressure of structure-zone diagram with the bombardment energy [438]. Though the combined Thorton-Messier diagram can successfully predict the microstructures of deposited thin films in many cases, the effects of ion bombardment rate such as ion/metal flux ratio are not considered or incorporated. The bombardment rate should be considered to be just as important as the bombardment energy during film deposition.

Fig. 59a-d show cross sectional SEM images of some typical microstructures of TiN and CrN thin films deposited on Si under ion beams of different energies with an incident angle of $45^{\circ}$ at 300 and $500{ }^{\circ} \mathrm{C}$, respectively $[424,426]$. Fig. 59a reveals that the microstructure of a TiN thin film deposited at $300{ }^{\circ} \mathrm{C}$ with lower ion/metal flux ratio $\left(J_{\mathrm{i}} / J_{\mathrm{Ti}}=1\right)$ and lower energy $(100 \mathrm{eV})$ consists of larger

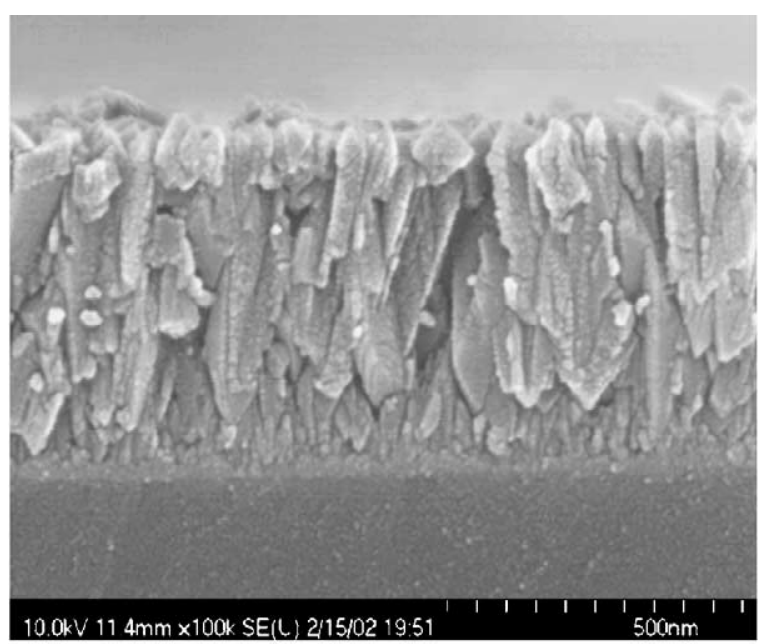

(a)

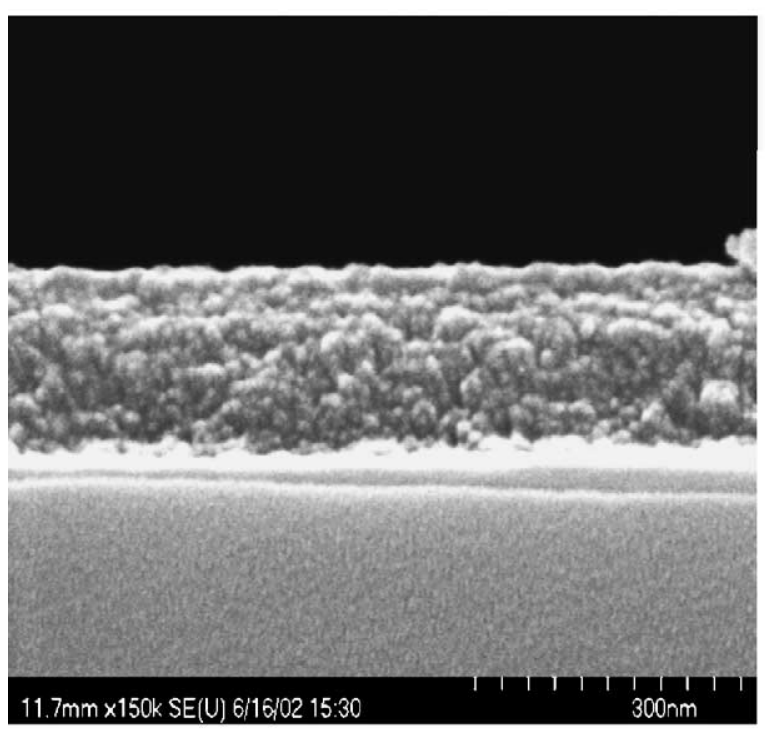

(c)

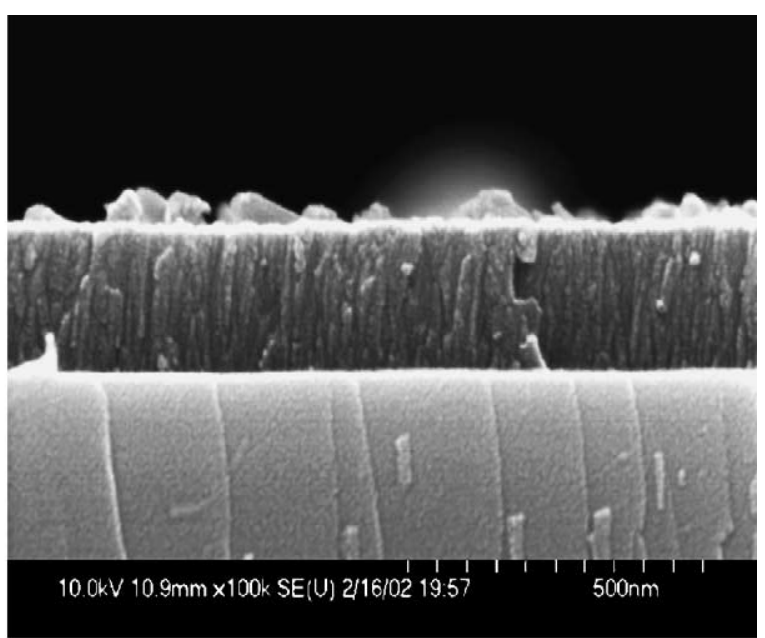

(b)

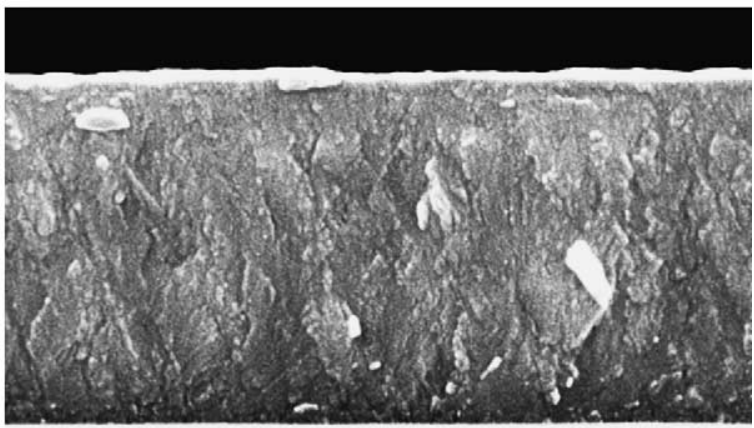

10.0kV $11.7 \mathrm{~mm} \times 80.0 \mathrm{k}$ SE(U) s00nm

(d)

Fig. 59. SEM microstructural micrographs of IBAD TiN and CrN thin films deposited on Si under ion angle incidence of $45^{\circ}$ at different ion energies and ion fluxes. (a) Zone I: TiN, $300{ }^{\circ} \mathrm{C}, 100 \mathrm{eV}, J_{\mathrm{i}} / J_{\mathrm{Ti}}=1$, (b) Zone T: TiN, $500{ }^{\circ} \mathrm{C}, 200 \mathrm{eV}, J_{\mathrm{i}} / J_{\mathrm{Ti}}=2$, (c) equiaxed grain: TiN, $500{ }^{\circ} \mathrm{C}, 500 \mathrm{eV}, J_{\mathrm{i}} / J_{\mathrm{Ti}}=2$, and (d) Zone II: CrN, $500{ }^{\circ} \mathrm{C}, 350 \mathrm{eV}, J_{\mathrm{i}} / J_{\mathrm{Cr}}=2[424,426]$. 
columnar grains with open boundaries (Zone I). Increasing the substrate temperature to $500{ }^{\circ} \mathrm{C}$, the $J_{\mathrm{i}} /$ $J_{\mathrm{Ti}}$ ratio to 2 , and the ion energy to $200 \mathrm{eV}$ results in the formation of dense and finely grained structure (Zone T; Fig. 59b). When the ion energy is increased to $500 \mathrm{eV}$, equiaxed grains are produced in TiN films (Fig. 59c). Thus, a new microstructure termed "equiaxed" or "granular" structure [440] is formed under higher ion/metal flux ratio $\left(J_{\mathrm{i}} / J_{\mathrm{Ti}}=2\right)$ and low homologous temperature $\left(T / T_{\mathrm{M}}<0.33\right)$. The formation of the equiaxed structure is attributed to the breakdown of the columnar structure by ion bombardment, which leads to renucleation and retarding of the columnar growth in the films [441]. From the work of Chen and coworkers [424,426], the effects of IBAD processing parameters on the microstructures of TiN, $\mathrm{VN}$ and $\mathrm{CrN}$ films deposited at 300 and $500{ }^{\circ} \mathrm{C}$ are summarized in Fig. $60 \mathrm{a}$ and b. The microstructures of nitrides are independent of incident angle of the ion beam. TiN, VN and $\mathrm{CrN}$ have significantly different melting temperatures (3563, 2353 and $1323 \mathrm{~K}$, respectively [442]), so even at the same deposition temperature such as $500{ }^{\circ} \mathrm{C}$, the homologous temperatures of these nitrides range from 0.22 to 0.58 . The predicted microstructures range from Zone I to Zone II in the ThortonMessier structure-zone diagram. The nanohardness of TiN thin films having (2 00 ) fiber orientation is determined to be $15 \mathrm{GPa}$, that is lower than that reported for ion plated TiN films with larger grain sizes [410,411]. Chen and coworkers attributed the softening of TiN films with an average grain size of $\sim 15 \mathrm{~nm}$ to the grain-boundary rotation or grain-boundary sliding assisted by grain-boundary diffusion $[424,426]$.

\subsection{Superhard and super-tough nanocrystalline coatings}

There is an increasing need in industrial sectors for the development of high-performance coatings with better oxidation resistance, higher hardness and longer lifetime than conventional TiN single layer coatings. To meet industrial demands for improved coatings, significant effort has been devoted to the design and synthesis of superhard coatings. Koehler [443] was the first to propose a concept for the design of strong solids or coatings by using two different alternate layers of materials with high and low elastic constants, respectively. The thickness of each layer should be in the nanometer range, and no dislocation source could operate within the layers. If the dislocations could form in the material layer with the lower modulus, they need to overcome a significant repulsive image stress from the higher modulus phase before slip can be transmitted across layers [443]. Thus, preventing slip from propagating through the whole film. Such multilayer coatings are often termed as "superlattices", and the bilayers of these superlattices can be metal layers, nitrides, or carbides. In designing multilayer coatings, both structural and functional components must be taken into consideration. The former involves the grain size, thickness of individual layers, composition modulation and the number of material interfaces whereas the latter is concerned with the wear, mechanical and physical characteristics. Many different hard materials can be combined in multilayer coatings resulting in optimized material properties. Fig. 50 shows potential hard materials for multilayer coatings [386,444]. Recent advances in coating technologies permit the deposition of multilayers with superior chemical and mechanical properties using PVD and plasma-assisted PVD [386]. Typical examples of these multilayered structures include $\mathrm{Al} / \mathrm{Cu}, \mathrm{Al} / \mathrm{Ag}$ [445,446], TiN/AlN [447], TiN/VN [448], TiN/NbN [449,450]. The hardness of TiN/VN and TiN/NbN coatings exceeds $50 \mathrm{GPa}$ when the superlattice bilayer period is around $5 \mathrm{~nm}$. The hardness of the superlattice coatings is more than twice that of $\mathrm{TiN}, \mathrm{VN}$ or $\mathrm{NbN}$ single layer films [448-450]. Chu et al. explained the hardness enhancement in TiN/NbN superlattice coatings based on restricted dislocation movement within and between layers in the coating. Their model predicts a peak maximum in hardness when there is a difference in shear modulus between the two materials and sharp interfaces between them [451]. In the case where the superlattice period of TiN/NbN larger than $5 \mathrm{~nm}$, the hardness of 
(a)
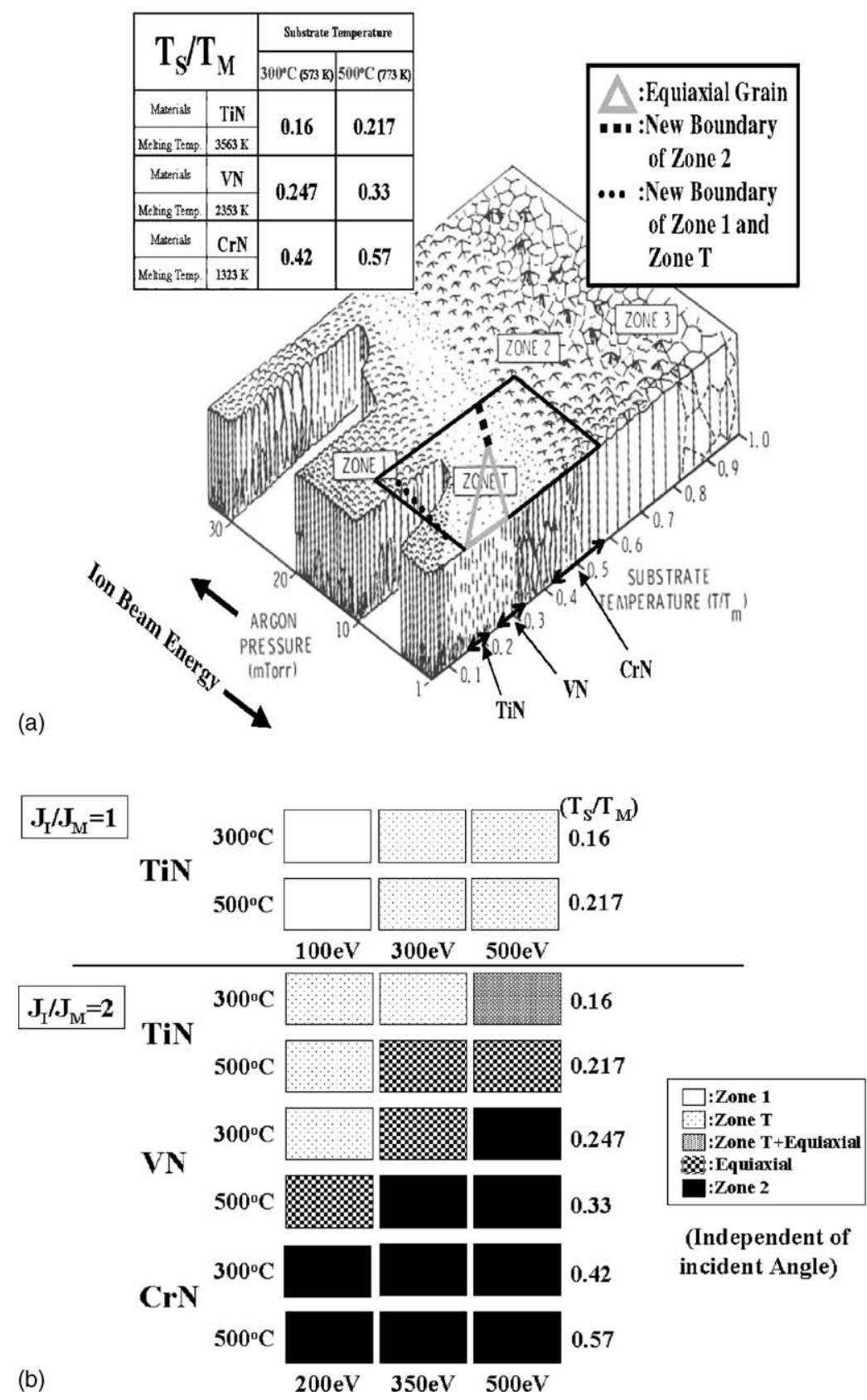

Fig. 60. (a) Thorton-Messier structure zone map for the IBAD TiN, VN and CrN thin films on Si substrates and (b) details of the structure zone maps for these nitrides. The substrate temperatures are 300 and $500{ }^{\circ} \mathrm{C}$, ion/atom ratio from 1 to 2 , and ion beam energy from 100 to $500 \mathrm{eV}[424,426]$.

superlattice decreases dramatically to $14 \mathrm{GPa}$ [452]. Fig. 61 shows the cross-sectional TEM images of $\mathrm{TiN} / \mathrm{NbN}$ multilayers deposited on a Si substrate in which the period of superlattice is $18.5 \mathrm{~nm}$. The layers can be easily recognized since TiN layers are visible as bright bands and $\mathrm{NbN}$ layers as dark ones. The new physical properties of multilayers enable them to be used in micro-electro-mechanical systems (MEMS) as small devices where the wear protection of rubbing elements is an importanat issue [452]. In a note appended in proof, Koehler indicated that one of the layers in superlattices should 

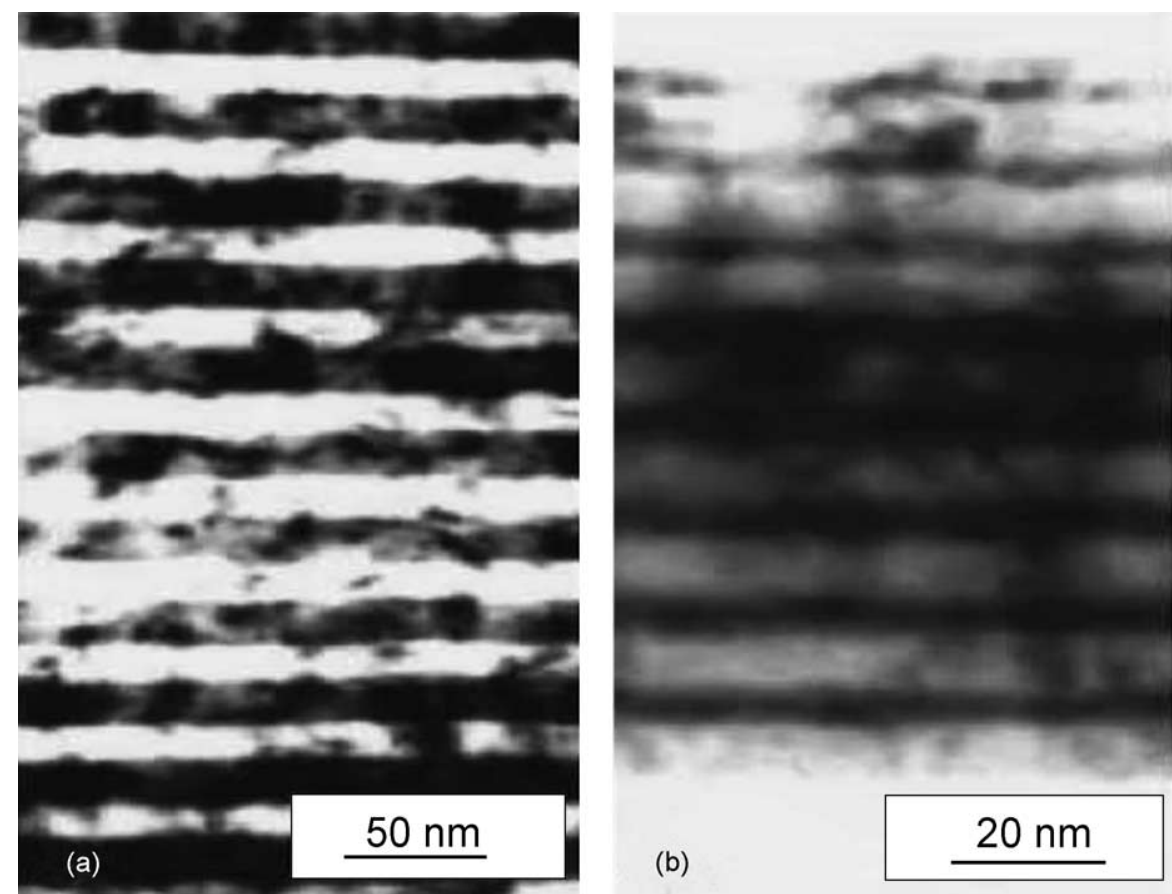

Fig. 61. Cross-sectional TEM images of TiN/NbN superlattices deposited on (a and b) an Si substrate (reprinted from [452] with permission from Elsevier).

be amorphous, because the amorphous material can more easily accommodate the lattice mismatch to the crystalline material [443].

From this aspect, Veprek et al. designed single layer nanocomposite coatings with hardness $\geq 40 \mathrm{GPa}$ using plasma CVD process either in a high frequency or direct current discharge [453462]. In the process, a hard transition metal nitride and a covalent nitride (e.g. $\mathrm{Si}_{3} \mathrm{~N}_{4}$, or $\mathrm{BN}$ ), are deposited simultaneously to form immiscible phases with interfaces having a high cohesive energy. In other words, the coatings consist of transition metal nitride nanocrystallites with grain sizes in the nanometer range $(\sim 4-6 \mathrm{~nm})$ embedded into $<1 \mathrm{~nm}$ thin matrix of amorphous matrix. Such a microstructure is now termed 'nanocomposite' coatings (Fig. 62). The coatings have a type $n c-\mathrm{M}_{n} \mathrm{~N} / \mathrm{a}-\mathrm{Si}_{3} \mathrm{~N}_{4}$ where nc- and a- denote the crystalline and amorphous phases, respectively, and $\mathrm{M}_{n}$ represents transition metals like Ti, W, V and Zr. In nanocomposite coatings, the transition metal nitride phase is sufficiently hard to bear the load whilst the amorphous nitride provides structural flexibility.

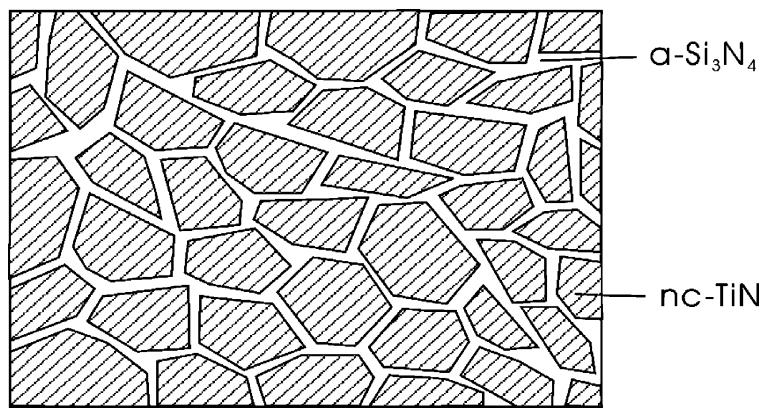

Fig. 62. Schematic representation of nc-TiN/a-Si ${ }_{3} \mathrm{~N}_{4}$ nanocomposite coating consisting of TiN nanocrystalline phase embedded in $\mathrm{a}-\mathrm{Si}_{3} \mathrm{~N}_{4}$ amorphous matrix. 
From molecular dynamics computer simulations, plastic deformation in nanocrystalline materials can be accommodated by grain-boundary mechanisms when the size of nanograins is below $10 \mathrm{~nm}$. In this case, grain-boundary sliding events controlled by grain-boundary diffusion are possibly responsible for plastic deformation in nanocrystaline materials. The sliding is triggered by atomic shuffling and to some extent by stress-assisted free volume migration [298,300,302]. In other words, bulk nanocrystalline materials exhibit softening behavior when the grain size gets extremely small. As such, further increase in hardness requires blocking of grain-boundary sliding. This is the case for nc$\mathrm{M}_{n} \mathrm{~N} / \mathrm{a}-\mathrm{Si}_{3} \mathrm{~N}_{4}$ system in which the hardness values for the nc-TiN/a-Si $\mathrm{N}_{4}$ and nc- $\mathrm{W}_{2} \mathrm{~N} / \mathrm{a}-\mathrm{Si}_{3} \mathrm{~N}_{4}$ nanocomposite coatings appear to increase with decreasing crystallite size down to $4 \mathrm{~nm}$. Veprek et al. reported that the hardness of these plasma CVD nanocomposite coatings could reach the diamond hardness (70-80 GPa) when the crystallite size approaches about $2 \mathrm{~nm}$ (Fig. 63). In order to achieve superhardness, the concentration of the $\mathrm{Si}_{3} \mathrm{~N}_{4}$ phase should be about 17-23 mol\%. This is due to the the development of nanograined TiN structure embedded in the a- $\mathrm{Si}_{3} \mathrm{~N}_{4}$ matrix $[455,458]$. The nc$\mathrm{M}_{n} \mathrm{~N} / \mathrm{a}-\mathrm{Si}_{3} \mathrm{~N}_{4}$ system shows remarkable thermal stability to high temperatures of up to $1000{ }^{\circ} \mathrm{C}$ [458]. The plasma CVD process provided the high chemical activity of the gas phase, and controlled surface mobility associated with ion bombardment. The disadvantages of plasma CVD have been described previously. PVD methods such as magnetron sputtering-assisted pulse laser deposition (MSPLD) can also be used to prepare other nanocrystalline/amorphous coatings such as TiC/amorphous-carbon (a$\mathrm{C}$ ) and WC/a-C with a unique combination of high hardness and toughness. The a-C acts as a hard, tough and lubricating matrix whereas nanoparticles act as reinforcing crystallites to improve hardness and other mechanical properties [463-465]. As the highly elastic nc- $\mathrm{M}_{n} \mathrm{~N} / \mathrm{a}-\mathrm{Si}_{3} \mathrm{~N}_{4}$ system generally exhibits brittle behavior, Voevodin et al. [463-465] designed super-tough coatings with larger nanocrystallites of 10-50 nm encapsuled in an amorphous matrix, separating the nanocrystals by a larger width of $5 \mathrm{~nm}$. This allows the formation of dislocations, but is too small for self-propagation of the cracks. The larger grain separation permits adaptation of incoherency strains and formation of

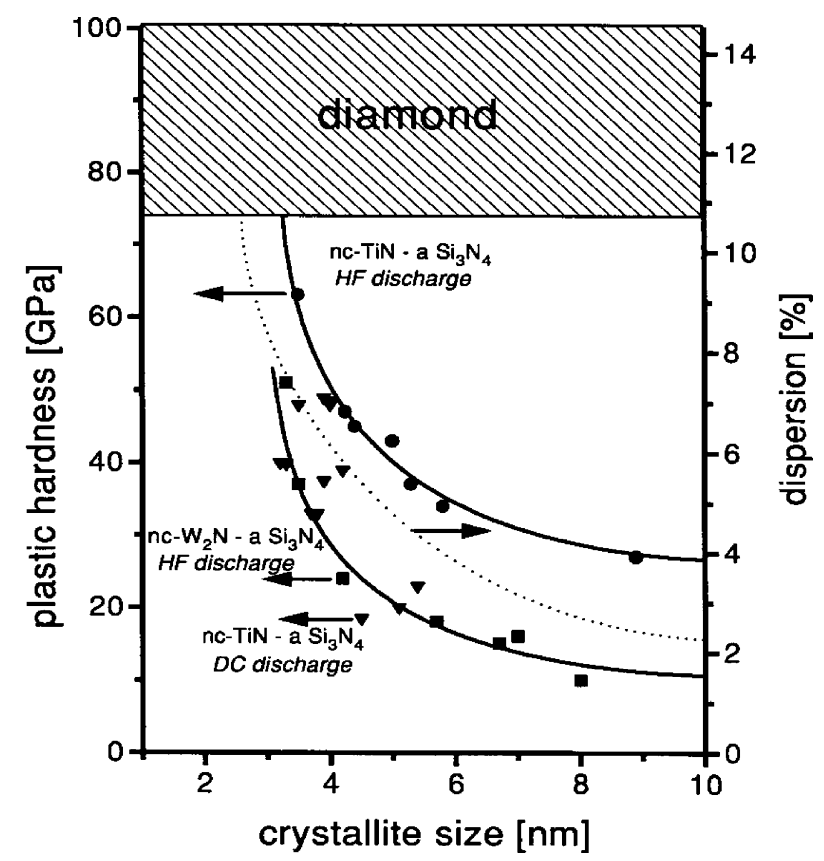

Fig. 63. Dependence of the measured hardness of the $n c-\mathrm{M}_{n} / \mathrm{a}-\mathrm{Si}_{3} \mathrm{~N}_{4}$ nanocomposite coatings (reprinted from [457] with permission from Elsevier). 
nanocracks between the crystallites, leading to the occurrence of plasticity. Accordingly, super-tough TiC/a-C coatings have a hardness of $30 \mathrm{GPa}$, friction coefficient of $0.15-0.2$ and their toughness is four times higher than that of nanorystalline TiC [463,464].

As discussed above, superlattice coating or multilayers are potential materials for MEMS applications. In general, silicon and other electronic materials have been used to fabricate miniature mechanical components (micromachines) such as membranes, cantilever beam, gears, motors, pumps and valves using standard process of the integrated circuit industry via surface machining [466]. Surface machining is a process for making surface structures from deposited thin films. Surface microstructures typically range from 0.1 to several micrometer in thickness with lateral dimensions of $10-500 \mu \mathrm{m}$ and are offset $0.1-2 \mu \mathrm{m}$ from the substrate. The surface micromachines have multi-layered structure consisting typical of silicon substrate, isolation and sacrificial layers. The substrate is initially coated with an isolation layer that protects it during subsequent etching steps followed by the deposition of sacrificial layer. Finally, microstructural thin film of polysilicon is deposited on the sacrificail layer via low-pressure chemical vapor deposition (LPCVD). Selective etching of the sacrificial layer creates the free-standing micromechanical structures [466,467]. The adhesion of the microstructure to adjacent surfaces can occur either during the final processes of the micromachining process, or after packaging of the device. This is generally referred to as stiction in MEMS [467]. The applications for current MEMS devices are limited because they are made exclusively from silicon having poor mechanical and tribological properties [468]. It is likely that hard, inorganic coatings or as structural layers can improve the wear performance of micromachines. These materials include silicon carbide, diamond and diamond-like carbon (DLC). Silicon carbide possesses low friction characteristic, and is second only to diamond in wear resistance. One of the main challenges limiting the widespread use of $\mathrm{SiC}$ has been integrating this material into $\mathrm{Si}$ fabrication technology for MEMS [466,469]. Diamond and DLC are promising anti-stiction coating materials for micromechanical devices. They have high elastic modulus and hardness, and chemically inert, and have excellent tribological characteristics. Similarly, the challenge with DLC utilization in micromachining is the actual integration of this coating into a microstructure. The fracture strength of diamond is 23 times that of $\mathrm{Si}$, and the projected wear life of diamond MEMS moving mechanical assemblies is 10,000 times greater than that of Si counterparts. However, diamond is difficult to fabricate, and conventional CVD diamond films have large grain size and internal stress [470].

Recently, Gruen and Carlisle of Argonne National Laboratory have synthesized ultrananocrystalline diamond (UNCD) coatings via microwave plasma chemical vapor deposition (MPCVD) technique using a unique $\mathrm{C}_{60} / \mathrm{Ar}$ or $\mathrm{CH}_{4}(99 \%) / \mathrm{Ar}(1 \%)$ chemistry [470-475]. In the latter case, carbon dimers $\left(\mathrm{C}_{2}\right)$ are generated from methane via the reactions,

$$
\begin{aligned}
& 2 \mathrm{CH}_{4} \rightarrow \mathrm{C}_{2} \mathrm{H}_{2}+3 \mathrm{H}_{2} \\
& 2 \mathrm{C}_{2} \mathrm{H}_{2} \rightarrow \mathrm{C}_{2}+\mathrm{H}_{2}
\end{aligned}
$$

in atmospheres containing very small quantities of hydrogen. In contrast, conventional CVD diamond film grown from $\mathrm{CH}_{4}(1 \%) / \mathrm{H}_{2}(99 \%)$ gas mixture containing excess of atomic hydrogen that etches the formed diamond phase steadily, resulting in the formation of columnar morphology with large grain size and high surface roughness [470]. The rough surface finish of microcrystalline diamond can cause severe abrasion during sliding against other materials. Gruen et al.used the term UNCD to distinguish this material with 2-5 $\mathrm{nm}$ grain size from microcrystalline diamond (MCD) (1-10 $\mu \mathrm{m}$ grain size) and nanocrystalline diamond (50-100 nm grain size). Fig. 64 shows the typical atomic force microscopy (AFM) data for MCD and UNCD thin films grown in microwave plasma [470]. It is evident that 


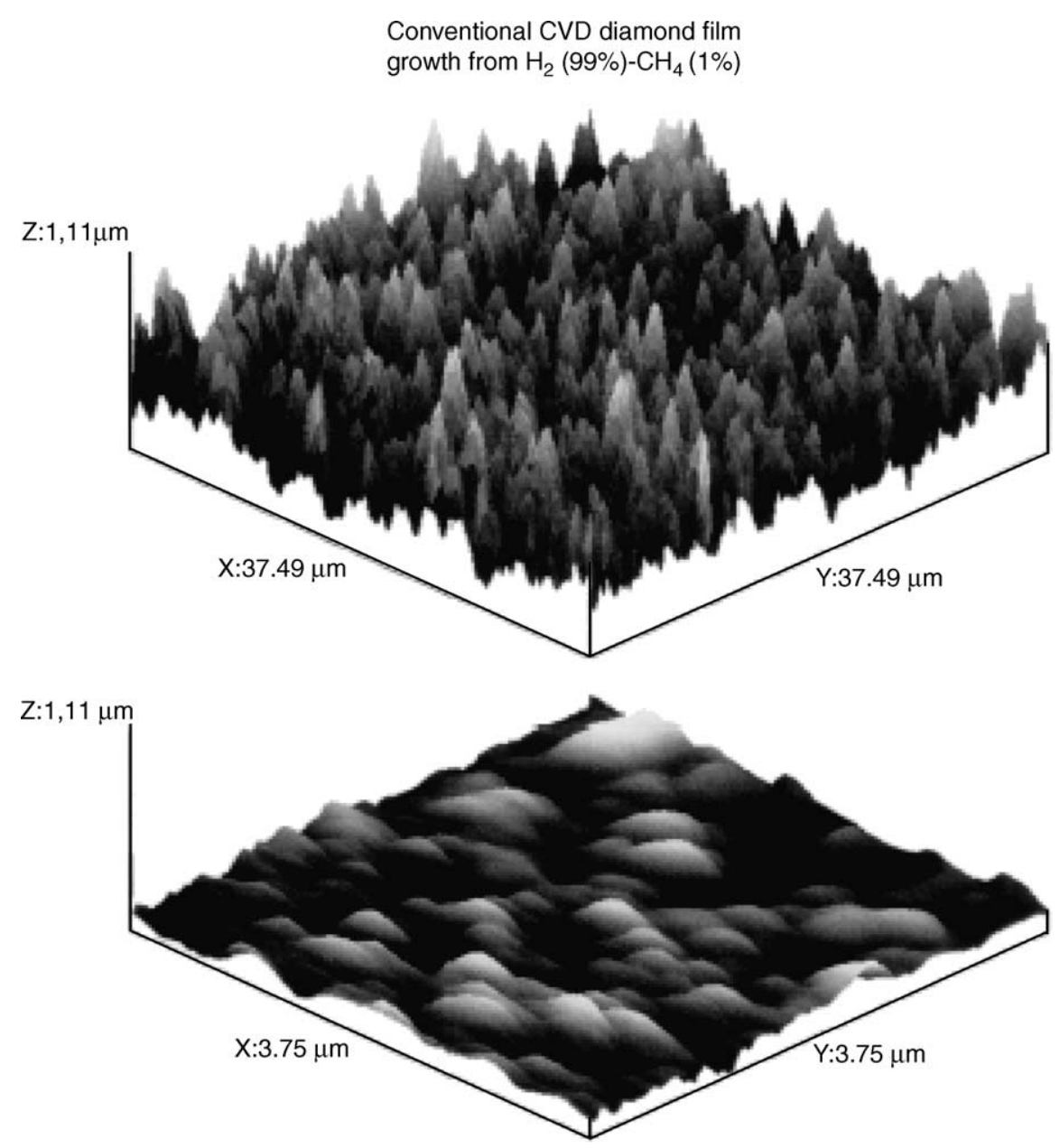

Nanocrystalline CVD diamond film growth from $\operatorname{Ar}(98 \%), \mathrm{H}_{2}(2 \%), \mathrm{C}_{60}$

Fig. 64. AFM surface morphologies of MCD and UNCD thins films (reprinted from [470] with permission from Elsevier).

UNCD exhibits smooth surface morphology. It is worth-noting that UNCD possesses hardness (88 GPa) and modulus nearly equivalent to single crystal diamond [470]. Moreover, the fracture strength of UNCD (4.13 GPa) far exceeds that of Si (0.3 GPa), SiC (1.2 GPa), DLC (0.7 GPa) and single crystal diamond (2.8 GPa) [471]. The friction coefficient of UNCD film is comparable with that of natural diamond, and wear damage on counterface materials is minimal owing to its smooth surface appearance. Consequently, UNCD films with improved mechanical and tribological properties are ideal materials for MEMS applications [470,472].

\section{Conclusions}

This paper provides a comprehensive state of the art review of the synthesis, structure, mechanical and physical properties of nanoparticles, nanocrystalline materials, and coatings. Nanocrystaline materials show great promise for use in structural and device applications in which 
enhanced mechanical and physical properties are required. Substantial progress has been made in the development, processing and microstructural aspects of nanoparticles and nanocrystalline materials over the recent years. Nanoparticles can be synthesized via vapor, liquid and solid state processing routes. Bulk nanocrystalline materials can be prepared via inert gas-phase condensation of nanoparticles, consolidation, mechanical alloying and compaction, electrodeposition and severe plastic deformation. Some details of controlled processing techniques, micro structures and mechanical properties of nanocrystalline materials have been addressed. In general, nanocrystalline metals exhibit extremely high yield strength and hardness, enhanced superplasticity at lower temperatures and higher strain rates, but low tensile ductility compared to their microcrystalline counterparts. Improvements in mechanical properties can be achieved through the retention of grain sizes in nanometer scale and appropriate microstructure control. The microstructure and properties of the nanocrystalline materials are strongly dependent on the synthesis and fabrication routes. There is a large variation of grain sizes and internal structures of nanocrystalline materials formed from different processing routes. The presence of other microstructural features such as nanovoids, impurities, etc can affect the mechanical strength and stiffness of nanocrystalline materials. Fundamental understanding of the relationships between the processing, microstructure, and failure mechanisms enables one to design nanocrystalline materials with superior mechanical properties and performances.

Refinement of grain sizes to nanometer regime leads to specific changes in the mechanisms of deformation owing to reduced availability of mobile dislocations. In contrast, plastic deformation of conventional microcrystalline metals depends mainly on the dislocation activities. Polycrystalline materials show an increase in strength and hardness with decreasing grain size according to the HallPetch (H-P) relationship. Enhanced strength and hardness has been observed in nanocrystalline materials with reducing grain sizes down to a critical value, i.e. ca. 10-20 nm. Below the critical grain size, a softening behavior or inverse $\mathrm{H}-\mathrm{P}$ relation is often observed. The explanations for inverse $\mathrm{H}-\mathrm{P}$ effect remain controversial. Several mechanisms have been proposed for such anomalous mechanical behavior. These include the presence of nanovoids, grain-boundary sliding, grain-boundary rotation, and Coble creep diffusion. The mechanism by which the softening takes place is poorly understood at present. This is attributed to limited or availability of appropriate experimental tools or routes to detect the mechanical responses of nanocrystalline materials below the critical grain size. The inability to obtain porous and contaminant-free material with a similar microstructure within a single processing method is another problem.

Computer simulations can provide the details of atomic level structure and deformation that are not accessible by experimental routes. Large scale molecular dynamics (MD) simulations have been carried out by several researchers to explain the deformation behavior of nanocrystalline materials with grain sizes below a critical value. These often predict mechanisms based on grain boundary sliding and Coble creep diffusion. The sliding is triggered by atomic shuffling and to some extent by stress-assisted free volume migration. However, molecular dynamics simulations also have certain limitations. MD simulations of the deformation of nanocrystalline materials are performed under high load and extremely high strain rate conditions. These impose restrictions on the number or the size of the grains to be simulated. The simulated properties of samples with a small number of grains may not represent the actual characteristics of real nanocrystalline materials. Further, the deformation can only be initiated at early stages, thus subsequent microstructural evolution and fracture damage resulting from prolonged deformation are excluded from the simulations. Nevertheless, MD simulations are complementary to the experimental techniques for exploring and explaining the deformation characteristics of nanocrystalline materials. With the advent of very powerful computers, it is expected that advanced numerical methods and computer simulations are becoming increasingly used for the modeling of microstructure and deformation characteristics of nanocrystalline materials. 
Nanocrystalline coatings with grain sizes in the nanometer scale can exhibit novel and enhanced properties, which opens up opportunities for new technological applications. Various techniques including thermal spraying, IBAD, PVD and plasma CVD have been used to fabricate nanocrystalline coatings for specific applications. The use of nanocrystalline coatings is still limited because applications require large scale control of the synthesis of nanoparticles. Thermal spraying offers the unique advantage of moderate to high rate of throughput and the ability to coat target materials with complex shapes using nanostructured feedstock powders prepared from vapor, liquid and solid routes. High velocity oxygen fuel thermal spraying is very effective to deposit dense nanocrystalline coatings with superior wear properties. IBAD with several controlled processing parameters allows the formation of nanocrystalline coatings based on transition metal nitrides with improved adhesion characteristics. Finally, multilayered coatings can provide hardness enhancements of more than twice that compared to single layer base materials. Multilayers are composed of many very thin alternating layers and their properties are determined by the superlattice period. Superlattice structures are formed by depositing thin layers of two materials with high and low elastic moduli. Superhardness occurs because the dislocations formed in the layer with lower elastic modulus are repelled and annihilated at the layer boundaries. The proper selection of hard materials with different bonding, structures and functions is critical for tailoring multilayered coatings with desired properties. More recently, UNCD with high hardness and excellent wear resistant properties has been developed. UNCD possesses hardness and modulus nearly equivalent to single crystal diamond. The friction coefficient of UNCD film is comparable with that of natural diamond, and wear damage on counterface materials is minimal owing to its smooth surface appearance. Thus, UNCD, superlattice and nanocomposite coating materials are promising materials to be used as rubbing elements of micro-electro-mechanical systems. The designing of nanocomposite coatings also necessitates consideration of several factors and application requirements. Superhard nanocomposite coatings consisting of a nanocrystalline transition metal nitride embedded in a thin amorphous covalent nitride matrix exhibit hardness values $\geq 40 \mathrm{GPa}$. The design concept of superhard nanocomposite coatings involves preventing the formation of dislocations in the nanocrystalline phase and blocking the grain-boundary sliding of nanograins.

As mentioned above, several procedures have been developed to fabricate nanomaterials that can be routinely prepared using conventional fabrication processes. However, the rapid pace of progress in nanomaterials and nanotechnology has been made possible because of improved methods of synthesis, processing, characterization and simulation. Advanced nanotechnology for the fabrication of electronic nanodevices and MEMS requires the controlled assembly of well-ordered structures and the design of wear resistant superhard nanomaterials. Many new and advanced nanostructured materials, devices and systems will be prepared in the next few years using a variety of new functional nanobuilding blocks based on nanoparticles, nanowires and nonotubes or their self-assemblies. The growing interests in the application and fundamental knowledge of nanocrystalline materials have also motivated the research into techniques for synthesizing new nanomaterials with better controlled properties and dimensions. Understanding the structure-property relationship of nanocrystalline materials is vital for successful nanoscale modification and manipulation of these materials. At present, basic knowledge relating to the mechanical behavior of nanomaterials with grain size $\leq 10 \mathrm{~nm}$ is derived from MD simulation.

However, simulations samples are fully dense and free from impurities with their nanograins separated by high angle boundaries. In practice, nanocrystalline samples contain impurities, and the mechanical properties of nanocrystalline materials are very sensitive to their initial micro structures and impurities present. The ability to prepare high purity nanomaterials at relatively low cost is a matter of concern to the researchers. Moreover, the main problem of nanocrystalline materials is their instability at moderate or high temperatures. The beneficial superplasctic properties achieved in bulk 
nanocrystalline materials at lower temperatures are offset by rapid grain growth of these nanograins during deformation. Thus, maintaining the stability of bulk nanocrystalline materials for structural applications is also one of the main challenges faced by the materials scientists. Therefore, materials scientists have an important role to play in designing, developing and modeling of new nanomaterials and their functional structures with superior desired properties for advanced engineering applications.

\section{Acknowledgement}

The work described in this paper was fully supported by grants from the City University of Hong Kong (project nos. 7001530 and 7001335).

\section{References}

[1] H. Gleiter, Prog. Mater. Sci. 33 (1989) 223.

[2] P.G. Sanders, J.G. Barker, J.R. Weertman, J. Mater. Res. 11 (1996) 311.

[3] Y.K. Huang, A.A. Menovsky, F.R. de Boer, Nanostruct. Mater. 2 (1993) 505.

[4] T. Castro, R. Reifenberger, E. Choi, R.P. Andres, Phys. Rev. B 42 (1990) 8548.

[5] G. Schmidt, in: K.J. Klabunde (Ed.), Nanoscale Materials in Chemistry, John Wiley \& Sons, New York, 2001 pp. $23-$ 24.

[6] L.D. Marks, Rep. Prog. Phys. 57 (1993) 603.

[7] M. Yacaman, in: T.E. Whyte, R.A. Betta, E.G. Deroduane, R.T. Baker (Eds.), Catalytic Materials, Relationship between Structure and Reactivity, American Chemical Society, New York, 1983, p. 341.

[8] J.M. Montejano-Carrizales, J.L. Moran-Lopez, Nanostruct. Mater. 1 (1992) 397.

[9] J.M. Montejano-Carrizales, F. Aguilera-Granja, J.L. Moran-Lopez, Nanostruct. Mater. 8 (1997) 269.

[10] Z.L. Wang, J. Phys. Chem. B 104 (2000) 1153.

[11] Y. Sun, Y. Xia, Science 298 (2002) 2176.

[12] G. Schmidt, Chem. Rev. 92 (1992) 1709.

[13] T.S. Ahmadi, Z.L. Wang, T.C. Green, A. Henglein, M.A. El-Sayed, Science 272 (1996) 1924.

[14] J.G. Allpress, J.V. Sanders, Surf. Sci. 7 (1967) 1.

[15] J. Xu, X.K. Sun, A.Q. He, W.D. Wei, W.X. Chen, Nanostruct. Mater. 3 (1993) 253.

[16] W. Krakow, Nanostruct. Mater. 3 (1993) 115.

[17] G. Diaz, M. Jose-Yacaman, Mater. Chem. Phys. 41 (1995) 240.

[18] Z.L. Wang, Characterization of Nanophase Materials, Wiley-VCH Verlag GmbH, Weinheim, Germany, 2000(pp. 3780, Chapter 3).

[19] Z.L. Wang, T.S. Ahmadi, M.A. El-Sayed, Surf. Sci. 380 (1997) 302.

[20] R.W. Siegel, Annu. Rev. Mater. Sci. 21 (1991) 559.

[21] R.W. Siegel, MRS Bull. 15 (1990) 60.

[22] G.T. Thomas, R.W. Siegel, J.A. Eastman, Scr. Metall. 24 (1990) 201.

[23] R.W. Siegel, Nanostruct. Mater. 3 (1991) 1.

[24] R.W. Siegel, G.J. Thomas, Ultramicroscopy 40 (1992) 376.

[25] R.W. Siegel, Mater. Sci. Eng. A 168 (1993) 189.

[26] E.A. Stern, R.W. Siegel, M. Newville, P.G. Sanders, D. Haskel, Phys. Rev. Lett. 75 (1995) 3874.

[27] M.R. Fitzsimmons, J.A. Eastman, M. Muller-Stach, G. Wallner, Phys. Rev. B 44 (1991) 2452.

[28] J.A. Eastman, M.R. Fitzsimmons, L.J. Thompson, Phil. Mag. B 66 (1992) 667.

[29] G.J. Thomas, R.W. Siegel, J.A. Eastman, Scr. Metall. Mater. 24 (1990) 201.

[30] X. Zhu, R. Birringer, U. Herr, H. Gleiter, Phys. Rev. B 35 (1987) 9085.

[31] T. Haubold, R. Birringer, B. Lengeler, H. Gleiter, Phys. Lett. A 135 (1989) 461.

[32] U. Herr, J. Jing, U. Gonser, H. Gleiter, Solid State Commun. 76 (1990) 192.

[33] P. Keblinsli, D. Wolf, S.R. Phillpot, H. Gleiter, Scr. Mater. 41 (1999) 631.

[34] J. Loffler, J. Weissmuller, Phys. Rev. B 52 (1995) 7076. 
[35] R.W. Siegel, Nanostruct. Mater. 4 (1994) 121.

[36] H. Gleiter, Nanostruct. Mater. 6 (1995) 3.

[37] H. Gleiter, Acta Mater. 48 (2000) 1.

[38] J.E. Burke, D. Turnbull, Progr. Met. Phys. 3 (1952) 220.

[39] A.J. Ardell, Acta Metall. 20 (1972) 601.

[40] T.R. Malow, C.C. Koch, Acta Mater. 45 (1997) 2177.

[41] M.C. Iordache, S.H. Whang, Z. Jiao, Z.M. Wang, Nanostruct. Mater. 11 (1999) 1343.

[42] J.E. Burke, Trans. Am. Inst. Min. Eng. 180 (1949) 73.

[43] F. Zhou, J. Lee, E.J. Lavernia, Scr. Mater. 44 (20012013) 2013.

[44] P. Knauth, A. Charai, P. Gas, Scr. Metall. Mater. 28 (1993) 325.

[45] U. Klement, U. Erb, A.M. El-Sherik, K.T. Aust, Mater. Sci. Eng. A 203 (1995) 177.

[46] N. Wang, Z. Wang, K.T. Aust, U. Erb, Acta Mater. 45 (1997) 1655.

[47] V.Y. Gertsman, R. Birringer, Scr. Metall. Mater. 30 (1994) 577.

[48] P.G. Sanders, G.E. Fougere, L.J. Thompson, J.A. Eastman, J.R. Weertman, Nanostruct. Mater. 8 (1997) 243.

[49] H.G. Jiang, M.L. Lau, E.J. Lavernia, Nanostruct. Mater. 10 (1998) 169.

[50] L. Lu, L.B. Wang, B.Z. Ding, K. Lu, Mater. Sci. Eng. A 286 (2000) 125.

[51] J. Eckert, J.C. Holzer, W.L. Johnson, J. Appl. Phys. 73 (1993) 131.

[52] C. Xiao, R.A. Mirshams, S.H. Whang, W.M. Yin, Mater. Sci. Eng. A 301 (2001) 35.

[53] W.M. Yin, S.H. Whang, Scr. Mater. 44 (2001) 569.

[54] R. Suryanarayanan, C.A. Frey, S.M.L. Sastry, B.E. Waller, W.E. Buhro, Mater. Sci. Eng. A 264 (1999) 210.

[55] M.M. Disko, M.J. Luton, H. Shuman, Ultramicroscopy 37 (1991) 202.

[56] V.L. Tellkamp, A. Melmed, E.J. Lavernia, Metal. Mater. Trans. A 32 (2001) 2335.

[57] H.P. Klug, L.E. Alexander, X-ray Diffraction procedures for Polycrystalline and Amorphous Materials, second ed., John Wiley \& Sons, New York, 1974 (pp. 618-687).

[58] P. Scherrer, Nachr. Gottingen 2 (1918) 98.

[59] G.K. Williamson, W.H. Hall, Acta Metall. 1 (1953) 22.

[60] B.E. Warren, B.L. Averbach, J. Appl. Phys. 21 (1950) 595.

[61] H.G. Jiang, M. Ruhle, E.J. Lavernia, J. Mater. Res. 14 (1999) 549.

[62] L.H. Qian, S.C. Wang, Y.H. Zhao, K. Lu, Acta Mater. 50 (2002) 3425.

[63] H.H. Tian, M. Atzmon, Phil. Mag. A 79 (1999) 1769.

[64] T. Inami, M. Kobiyama, S. Okuda, H. Maeta, H. Ohtsuka, Nanostruct. Mater. 12 (1999) 657.

[65] Y.H. Zhao, H.W. Sheng, K. Lu, Acta Mater. 49 (2001) 365.

[66] Z. Zhang, F. Zhou, E.J. Lavernia, Metall. Mater. Trans. A 34 (2003) 1349.

[67] T. Ungar, Adv. Eng. Mater. 5 (2003) 323.

[68] G.E. Fougere, J.R. Weertman, R.W. Siegel, Nanostruct. Mater. 5 (1995) 127.

[69] P.G. Sanders, J.A. Eastman, J.R. Weertman, Acta Mater. 45 (1997) 4019.

[70] N. Guillou, L.C. Nistor, H. Fuess, H. Hahn, Nanostruct. Mater. 8 (1997) 545.

[71] R.W. Siegel, S. Ramasamy, H. Hahn, Z. Li, T. Lu, R. Gronsky, J. Mater. Res. 3 (1988) 1367.

[72] T. Haubolt, R. Bohn, R. Birringer, H. Gleiter, Mater. Sci. Eng. 153 (1992) 679.

[73] J.C. Sanchez-Lopez, A. Justo, A. Fernandez, Phil. Mag. B 76 (1997) 663.

[74] S. Okuda, M. Kobiyama, T. Inami, S. Takamura, Scr. Metall. 44 (2001) 2009.

[75] C.G. Granqvist, R.A. Buhrman, J. Appl. Phys. 47 (1976) 2200.

[76] H. Gleiter, in: N. Hansen, A. Horsewell, T. Lefferes, H. Lilholt (Eds.), Deformation of Polycrystals: Mechanisms and Microstructures, Riso National laboratory, Roskilde, Denmark, 1981, pp. 15-21.

[77] R.C. Flagan, M.M. Lundes, Mater. Sci. Eng. A 204 (1995) 113.

[78] H. Gleiter, Prog. Mater. Sci. 33 (1989) 223.

[79] A.R. Tholen, Mater. Sci. Eng. A 168 (1993) 131.

[80] J. Bigot, A.G. Goursat, G. Vernet, J.F Rimpert, J. Foulard, T. Sarle, French Patent \#Z8307414 (1983).

[81] Y. Champion, J. Bigot, Mater. Sci. Eng. A 217-218 (1996) 58.

[82] Y. Champion, J. Bigot, Nanostruct. Mater. 10 (1998) 1097.

[83] G.M. Chow, C.L. Chien, A.S. Edelstein, J. Mater. Res. 6 (1991) 8.

[84] G.M. Chow, A. Pattnaik, T.E. Schlesinger, R.C. Cammarata, M.E. Twigg, A.S. Edelstein, J. Mater. Res. 6 (1991) 737.

[85] F.H. Kaatz, G.M. Chow, A.S. Edelstein, J. Mater. Res. 8 (1993) 995.

[86] H. Hahn, R.S. Averback, J. Appl. Phys. 67 (1990) 1113.

[87] G.M. Chow, C.L. Chien, A.S. Edelstein, J. Mater. Res. 6 (1991) 8. 
[88] J. Ying, J. Aerosol. Sci. 24 (1993) 315.

[89] D.H. Pearson, A.S. Edelstein, Nanostruct. Mater. 11 (1999) 1111.

[90] G. Gonzalez, J.A. Freites, C.E. Rojas, Scr. Mater. 44 (2001) 1883.

[91] P. Taneja, R. Chandra, R. Banerjee, P. Ayyub, Scr. Mater. 44 (2001) 1915.

[92] V. Hass, R. Biringer, Nanostruct. Mater. 1 (1992) 491.

[93] T. Yamamoto, J. Mazumder, Nanostruct. Mater. 7 (1996) 305.

[94] M.F. Becker, J.R. Brock, H. Cai, D.E. Henneke, J.W. Keto, J. Lee, W.T. Nichols, H.D. Glicksman, Nanostruct. Mater. 10 (1998) 853.

[95] H. Ferkel, Nanostruct. Mater. 11 (1999) 595.

[96] E. Ozawa, Y. Kawakami, T. Seto, Scr. Mater. 44 (2001) 2279.

[97] N. Koshizaki, A. Narazaki, T. Sasaki, Scr. Mater. 44 (2001) 1925.

[98] M.S. El-Shall, W. Slack, W. Vann, D. Kane, D. Haneley, J. Phys. Chem. 98 (1994) 3067.

[99] M.I. Baraton, M.S. El-Shall, Nanostruct. Mater. 6 (1995) 301.

[100] M.L. Hitchman, K.F. Jensen, Chemical Vapor Deposition—Principles and Applications, Academic Press, London, 1993.

[101] A. Sherman, Chemical Vapor Deposition For Microelectronics—Principles, Technology and Applications, Noyes Publications, New Jersey, 1987.

[102] H.L. Lai, N.B. Wong, X.T. Zhou, H.Y. Peng, F.C. Au, N. Wang, I. Bello, C.S. Lee, S.T. Lee, X.F. Duan, Appl. Phys. Lett. 76 (2000) 294.

[103] E.W. Wong, P.E. Sheehan, C.M. Lieber, Science 277 (1997) 1971.

[104] J.Y. Seo, S.Y. Yoon, K. Niihara, K.H. Kim, Thin Solid Films 406 (2002) 138.

[105] H. Murakami, M. Hirakawa, C. Tanaka, Y. Yamakawa, Appl. Phys. Lett. 76 (2000) 1776.

[106] C. Bower, O. Zhou, W. Zhu, D.J. Werder, S. Jin, Appl. Phys. Lett. 77 (2000) 2767.

[107] M. Menon, D. Srivastava, J. Mater. Res. 13 (1998) 2357.

[108] M.M.J. Treasy, T.W. Ebbesen, J.M. Gibsen, Nature 381 (1996) 678.

[109] O. Lourie, H.D. Wagner, J. Mater. Res. 13 (1998) 2418.

[110] E.W. Wong, P.E. Sheehan, C.M. Lieber, Science 277 (1997) 1971.

[111] J.P. Lu, Phys. Rev. Lett. 79 (1997) 1297.

[112] D. Golberg, Y. Bando, P. Dorozhkin, Z.C. Dong, MRS Bull. 29 (2004) 38.

[113] G.G. Tibbetts, J. Cryst. Growth 66 (1984) 632.

[114] G.G. Tibbetts, Appl. Phys. Lett. 42 (1983) 666.

[115] G.G. Tibbetts, Carbon Fibers, Filaments and Composites, Kluwer Academic Publishers, Amsterdam, 1990 (pp. $73-$ 94).

[116] R.T.K. Baker, Carbon 27 (1989) 315.

[117] X. Wang, Z. Hu, X. Chen, Y. Chen, Scr. Mater. 44 (2001) 1567.

[118] Y.H. Tang, Y.F. Zhang, N. Wang, C.S. Lee, X.D. Han, I. Bello, S.T. Lee, Appl. Phys. Lett. 85 (1999) 7981.

[119] R.S. Wagner, W.C. Ellis, Appl. Phys. Lett. 4 (1964) 89.

[120] E.I. Givargizov, J. Cryst. Growth 20 (1973) 217.

[121] M. Morales, C.M. Lieber, Science 279 (1998) 208.

[122] X. Duan, C.M. Lieber, Adv. Mater. 12 (2000) 298.

[123] M.S. Gudiksen, J. Wang, C.M. Lieber, J. Phys. Chem. B 105 (2001) 4062.

[124] C.L. Cheung, A. Kurtz, H. Park, C.M. Lieber, J. Phys. Chem. B 106 (2002) 2429.

[125] D. Wang, F. Qian, C. Yang, Z. Zhong, C.M. Lieber, Nano Lett. 4 (2004) 871.

[126] Y. Wu, P. Yang, J. Am. Chem. Soc. 123 (2001) 3165.

[127] X.M. Meng, J. Liu, Y. Jiang, W.W. Chen, C.S. Lee, I. Bello, S.T. Lee, Chem. Phys. Lett. 382 (2003) 434.

[128] C.C. Chen, C.C. Yeh, Adv. Mater. 12 (2000) 738.

[129] Y.W. Wang, L.D. Zhang, C.H. Liang, G.Z. Wang, X.S. Peng, Chem. Phys. Lett. 357 (2002) 314.

[130] M.H. Huang, Y. Wu, H. Feick, E. Weber, P.D. Yang, Adv. Mater. 13 (2000) 113.

[131] H.S. Tsai, G.J. Jaw, S.H. Chang, C.C. Cheng, C.T. Lee, H.P. Liu, Surf. Coat. Technol. 132 (2000) 158.

[132] P. Pan, W. Berry, J. Electrochem. Soc. 132 (1980) 3001.

[133] S. Veprek, Thin Solid Films 130 (1985) 135.

[134] P. Heszler, L. Landstrom, M. Lindstam, J.O. Carlsson, J. Appl. Phys. 89 (2001) 3967.

[135] E. Borsella, S. Botti, R. Alexandrescu, I. Morjan, T. Dikonimos-Makris, R. Giorgi, S. Martelli, Mater. Sci. Eng. A 168 (1993) 177.

[136] Y.L. Li, Y. Liang, K.S. Xiao, R. Zheng, Z.Q. Hu, Mater. Lett. 22 (1995) 87. 
[137] Z.Y. Ma, S.C. Tjong, Y.L. Li, Compos. Sci. Technol. 59 (1999) 263.

[138] G.L. Messing, S.C. Zhang, G.V. Jayanthi, J. Am. Ceram. Soc. 76 (1993) 2707.

[139] M.R. Zachariah, M.I. Aquino, R.D. Shull, E.B. Steel, Nanostruct. Mater. 5 (1995) 383.

[140] J. Karthikeyan, C.C. Berndt, J. Tikkanen, J.Y. Wang, A.H. King, H. Herman, Nanostruct. Mater. 8 (1997) 61.

[141] H. Hahn, Nanostruct. Mater. 9 (1997) 3.

[142] A. Singhal, G. Skandan, N. Glumac, B.H. Kear, Scr. Mater. 44 (2001) 2203.

[143] J.H. Yu, J.S. Lee, K.H. Ahn, Scr. Mater. 44 (2001) 2213.

[144] W. Chang, G. Skandan, H. Hahn, S.C. Danforth, B.H. Kear, Nanostruct. Mater. 4 (1994) 345.

[145] W. Chang, G. Skandan, S.C. Danforth, B.H. Kear, Nanostruct. Mater. 4 (1994) 507.

[146] M. Ebelman, Ann. Chim. Phys. 16 (1846) 129.

[147] A. Cossa, Il Nuevo Cimento 3 (1870) 228.

[148] A. Stock, K. Somieski, Ber. Chem. Ges. 54 (1921) 740.

[149] N. Tohge, G.S. Moore, J.D. Mackenzie, J. Non-Cryst. Solids 63 (1984) 95.

[150] C.J. Brinker, J.W. Scherer, Sol-Gel Science: The Physics and Chemistry of Sol-gel Processing, Academic Press, Boston, 1990.

[151] A.C. Pierre, Introduction to Sol-Gel Processing, Kluwer Academic Publishers, Boston, 1998.

[152] B.E. Yoldas, J. Mater. Sci. 21 (1986) 1080.

[153] C.J. Brinker, K.D. Keefer, D.W. Schaefer, R.A. Assink, B.D. Kay, C.S. Ashley, J. Non-Cryst. Solids 63 (1984) 45.

[154] R.K. Iler, The Chemistry of Silica, Wiley, New York, 1979.

[155] S. Sakka, in: M.A. Aegerter, M. Jafelicci, Jr., D.F. Souza, E.D. Zanotto (Eds.), Sol-Gel Science and Technology, World Scientific, Singapore, 1989, pp. 76-102.

[156] C.J. Brinker, D.W. Keefer, D.W. Schaefer, C.S. Ashley, J. Non-Cryst. Solids 48 (1982) 47.

[157] S.J. Teichner, G.A. Nicolaon, M.A. Vicarini, G.E. Gardes, Adv. Colloid Interface Sci 5 (1976) 245.

[158] S. Utamapanya, K.J. Klabunde, J.R. Schlup, Chem. Mater. 3 (1991) 175.

[159] J. Fricke, Aerogels, Springer-Verlag, New York, 1986.

[160] E. Duval, A. Boukenter, T. Achibat, B. Champagnon, J. Serughetti, J. Dumas, Phil. Mag. B 65 (1992) 181.

[161] D.C.L. Vasconcelos, W.R. Campos, V. Vasconcelos, W.L. Vasconcelos, Mater. Sci. Eng. A 334 (2002) 53.

[162] H.D. Jang, Powder Technol. 119 (2001) 102.

[163] T.H. Liou, Mater. Sci. Eng. A 364 (2004) 313.

[164] G. Skandan, Y.J. Chen, N. Glumac, B.H. Kear, Nanostruct. Mater. 11 (1999) 149.

[165] T. Kozakura, M. Susumu, H. Iwaki, Jpn. Patent 49036876 (1974).

[166] B. Samuneva, V. Kozhukharov, C. Trapalis, R. Kranold, J. Mater. Sci. 28 (1993) 2353.

[167] K. Kamiya, K. Tanimoto, T. Yoko, J. Mater. Sci. Lett. 5 (1986) 402.

[168] A. Makishima, M. Asami, K. Wada, J. Non-Cryst. Solids 100 (1988) 321.

[169] Y. Nosaka, M. Jimbo, M. Aizawa, N. Fuji, R. Igarashi, J. Mater. Sci. Lett. 10 (1991) 406.

[170] J. Livage, M. Henry, C. Sanchez, Prog. Solid State Chem. 18 (1988) 259.

[171] F. Babonneau, A. Leaustic, J. Livage, Mater. Res. Soc. Symp. Proc. 121 (1988) 317.

[172] O. Masson, V. Rieux, R. Guinebretiere, A. Dauger, Nanostruct. Mater. 7 (1996) 725.

[173] X.Z. Ding, X.H. Liu, Mater. Sci. Eng. A 224 (1997) 210.

[174] C.H. Shek, J.K.L. Lai, G.M. Lin, Nanostruct. Mater. 7 (1999) 887.

[175] S.V. Manorama, C.V. Gopal Reddy, V.J. Rao, Nanostruct. Mater. 11 (1999) 643.

[176] Y. Xu, G. Huang, H. Long, Mater. Lett. 57 (2003) 3570.

[177] X.G. Tang, H.K. Guo, Q.F. Zhou, J.X. Zhang, Nanostruct. Mater. 10 (1998) 161.

[178] Z. Zhai, H. Chen, J. Appl. Phys. 94 (2003) 589.

[179] G. Piccaluga, A. Corrias, G. Ennas, A. Musinu, Mater. Sci. Found. 13 (2000) 1.

[180] Y.L. Huang, D.S. Xue, P.H. Zhou, Y. Ma, F.S. Li, Mater. Sci. Eng. A 359 (2003) 332.

[181] S. Bruni, F. Cariati, M. Casu, A. Lai, A. Musinu, G. Piccaluga, S. Solinas, Nanostruct. Mater. 11 (1999) 573.

[182] V. Vendange, Ph. Colomban, Mater. Sci. Eng. A 168 (1993) 199.

[183] F. Hatekeyama, S. Kanzaki, J. Am. Ceram. Soc. 73 (1990) 2107.

[184] X.C. Yang, Z.S. Ding, J. Mater. Res. 15 (2000) 2140.

[185] G. Padmaja, G. Mukunda, J. Am. Ceram. Soc. 82 (1999) 1393.

[186] C.M. Lieber, E.W. Wong, H. Dai, B.W. Maynor, L.D. Burns, Materials Research Society Symposium Proceedings, 1996, pp. 103-111.

[187] E.W. Wong, B.W. Maynor, L.D. Burns, C.M. Lieber, Chem. Mater. 8 (1996) 2041.

[188] H. Hoffmann, P. Bowen, N. Jongen, J. Lemaire, Scr. Mater. 44 (2001) 2197. 
[189] Y. Li, Y. Fan, Y. Chen, J. Mater. Chem. 12 (2002) 1387.

[190] D. Deng, M.J. Hampden-Smith, Chem. Mater. 5 (1993) 681.

[191] E. Lima Jr., V. Drago, R. Bolsoni, P.F. Fichtner, Solid State Commun. 125 (2003) 265.

[192] C.Y. Huang, H.J. Chiang, J.C. Huang, S.R. Sheen, Nanostruct. Mater. 8 (1998) 1393.

[193] R. Oshima, T. Yamamoto, Y. Mizukoshi, Y. Nagata, Y. Maeda, Nanostruct. Mater. 12 (1999) 111.

[194] K.S. Suslick, S.B. Choe, A.A. Cichowlas, M.W. Grinstaff, Nature 353 (1991) 414.

[195] K.E. Gonsalves, S.P. Rangarajan, A. Garcia-Ruitz, C.C. Law, J. Mater. Sci. Lett. 15 (1996) 1261.

[196] X. Cao, R. Prozovov, Y. Koltypin, G. Kataby, A. Gedaken, J. Mater. Res. 12 (1997) 402.

[197] N.A. Dhas, A. Gedanken, J. Phys. Chem. 101 (1997) 9495.

[198] J.S. Benjamin, Metall. Trans. 1 (1970) 2943.

[199] C.C. Koch, Nanostruct. Mater. 2 (1993) 109.

[200] B.S. Murty, S. Ranganathan, Int. Mater. Rev. 43 (1998) 101.

[201] J. He, J.M. Schoenung, Mater. Sci. Eng. A 336 (2002) 274.

[202] R.B. Schwarz, W.L. Johnson, Phys. Rev. Lett. 51 (1983) 415.

[203] T.D. Shen, C.C. Koch, T.L. McCormick, R.J. Nemanich, J.Y. Huang, J.G. Huang, J. Mater. Res. 10 (1995) 139.

[204] E. Hellstern, H.J. Fecht, Z. Fu, W.L. Johnson, J. Appl. Phys. 65 (1989) 305.

[205] H.J. Fecht, E. Hellstern, Z. Fu, W.L. Johnson, Metall. Trans. A 21 (1990) 2333.

[206] J. Eckert, J.C. Holzer, C.E. Krill III, W.L. Johnson, J. Mater. Res. 7 (1992) 1751.

[207] H.J. Fecht, Nanostruct. Mater. 6 (1995) 33.

[208] D. Oleszak, P.H. Shingu, J. Appl. Phys. 79 (1996) 2975.

[209] R. Kalyanaraman, S. Yoo, M.S. Krupashankara, T.S. Sundarshan, R.J. Dowding, Nanostruct. Mater. 10 (1998) 1379.

[210] S. Hwang, C. Nishimura, P.G. Mccormick, Mater. Sci. Eng. A 318 (2001) 22.

[211] X. Zhang, H. Wang, M. Kassem, J. Narayan, C.C. Koch, J. Mater. Res. 16 (2001) 3485.

[212] X. Zhang, H. Wang, M. Kassem, J. Narayan, C.C. Koch, Scr. Mater. 46 (2002) 661.

[213] X. Zhang, H. Wang, R.O. Scattergood, J. Narayan, C.C. Koch, Mater. Sci. Eng. A 344 (2003) 175.

[214] T.D. Shen, C.C. Koch, Acta Mater. 44 (1996) 753.

[215] B.L. Huang, R.J. Perez, E.J. Lavernia, M.J. Luton, Nanostruct. Mater. 7 (1996) 67.

[216] C.C. Koch, Nanostruct. Mater. 9 (1997) 13.

[217] F.J. Humphreys, M. Hatherly, Recrystallization and Related Annealing Phenomena, Pergamon, Oxford, UK, 1996.

[218] F. Zhou, X.Z. Liao, Y.T. Zhu, S. Dallek, E.J. Lavernia, Acta Mater. 51 (2003) 2777.

[219] B. Huang, R.J. Perez, E.J. Lavernia, Mater. Sci. Eng. A 255 (1998) 124.

[220] J. Ding, T. Tsuzuki, P.G. McCormick, R. Street, J. Phys. D 29 (1996) 2365.

[221] E. Baburaj, K. Hubert, F. Froes, J. Alloys Compd. 257 (1997) 146.

[222] J. Ding, W.F. Miao, P.G. McCormick, R. Street, Appl. Phys. Lett. 67 (1995) 3804.

[223] J. Ding, T. Tsuzuki, P.G. McCormick, Nanostruct. Mater. 8 (1997) 75.

[224] A.C. Dodd, K. Raviprasad, P.G. McCormick, Scr. Mater. 44 (2001) 689.

[225] T. Tsuzuki, P.G. McCormick, Scr. Mater. 44 (2001) 1731.

[226] Z. Brankovic, G. Brankovic, C. Jovalekic, Y. Maniette, M. Cilense, J.A. Varela, Mater. Sci. Eng. A 345 (2003) 243.

[227] L.M. Cukrov, T. Tsuzuki, P.G. McCormick, Scr. Mater. 44 (2001) 1787.

[228] J. Rawers, G. Slavens, R. Krabbe, Nanostruct. Mater. 9 (1997) 197.

[229] K.I. Moon, H.S. Park, K.S. Lee, Mater. Sci. Eng. A 323 (2002) 293.

[230] G. Liang, Q. Meng, Z. Li, E. Wang, Nanostruct. Mater. 5 (1995) 673.

[231] J. Rawers, R.A. Krabbe, J.D. Wittenberger, Scr. Mater. 37 (1997) 2053.

[232] M.S. Haji-Mahmood, L.S. Chumbley, Nanostruct. Mater. 7 (1996) 95.

[233] Z. Livne, A. Munitz, J.C. Rawers, R.J. Fields, Nanostruct. Mater. 10 (1998) 503.

[234] V.L. Tellkamp, E.J. Lavernia, Nanostruct. Mater. 12 (1999) 249.

[235] T.M. Lillo, G.E. Korth, Nanostruct. Mater. 10 (1998) 35.

[236] J. Rawers, Nanostruct. Mater. 11 (1999) 513.

[237] W.H. Gourdin, J. Appl. Phys. 55 (1984) 172.

[238] G.E. Korth, R.L. Williamson, Met. Mater. Trans. A 26 (1995) 2571.

[239] M. Jain, T. Christman, Acta Mater. 42 (1994) 1901.

[240] S.C. Glade, N.N. Thadhani, Metall. Trans. A 26 (1995) 2565.

[241] R. Chau, M.B. Maple, W.J. Nellis, J. Appl. Phys. 79 (1996) 9236.

[242] J.C. Rawers, G. Korth, Nanostruct. Mater. 7 (1996) 25.

[243] J. Bystrzycki, J. Paszula, R.A. Varin, Mater. Sci. Eng. A 239-240 (1997) 546. 
[244] T. Mashimo, X. Huang, S. Hirosawa, K. Makita, S. Mitsudo, M. Motokawa, J. Mater. Proc. Technol. 85 (1999) 138.

[245] R.A. Varin, L. Zbroniec, T. Czujko, Y.K. Song, Mater. Sci. Eng. A 300 (2001) 1.

[246] I.V. Alexandrov, Y.T. Zhu, T.C. Lowe, R.K. Islamgaliev, R.Z. Valiev, Nanostruct. Mater. 10 (1998) 45.

[247] R.Z. Valiev, R.K. Islamgaliev, I.V. Alexandrov, Prog. Mater. Sci. 45 (2000) 103.

[248] L.S. Toth, Adv. Eng. Mater. 5 (2003) 308.

[249] K. Neishi, Z. Horita, T.G. Langdon, Mater. Sci. Eng. A 325 (2002) 54.

[250] S. Komura, Z. Horita, M. Nemoto, T.G. Langdon, J. Mater. Res. 14 (1999) 4044.

[251] R.Z. Valiev, I.V. Alexandrov, Nanostruct. Mater. 12 (1999) 35.

[252] V.A. Teplov, V.P. Pilugin, V.S. Gaviko, E.G. Chernyshov, Nanostruct. Mater. 6 (1995) 437.

[253] R.S. Mishra, V.V. Stolyarov, C. Echer, R.Z. Valiev, A.K. Mukherjee, Mater. Sci. Eng. A 298 (2001) 44.

[254] T. Mukai, M. Kawazoe, K. Higashi, Nanostruct. Mater. 10 (1998) 755.

[255] H. Hasegawa, S. Komura, A. Utsunomiya, Z. Horita, M. Furukawa, M. Nemoto, T.G. Langdon, Mater. Sci. Eng A 265 (1999) 188.

[256] R.K. Islamgaliev, N.F. Yunusova, I.N. Sabirov, A.V. Sergueeva, R.Z. Valiev, Mater. Sci. Eng. A 319-321 (2001) 877.

[257] S. Lee, M. Furukawa, Z. Horita, T.G. Langdon, Mater. Sci. Eng. A 342 (2002) 295.

[258] S. Lee, A. Utsunomiya, H. Akamatsu, K. Neishi, M. Furukawa, Z. Horita, T.G. Langdon, Acta Mater. 50 (2002) 553.

[259] K.T. Park, D.Y. Hwang, Y.K. Lee, Y.K. Kim, D.H. Shin, Mater. Sci. Eng. A 341 (2003) 273.

[260] J.Y. Chang, A. Shan, Mater. Sci. Eng. A 347 (2003) 165.

[261] V.V. Stolyarov, R. Lapovok, I.G. Brodova, P.F. Thomson, Mater. Sci. Eng. A 357 (2003) 159.

[262] C. Xu, M. Furukawa, Z. Horita, T.G. Langdon, Adv. Eng. Mater. 5 (2003) 359.

[263] U. Erb, A.M. El-Sherik, US Patent 5,352,266 (1994).

[264] A.M. El-Sherik, E. Erb, J. Mater. Sci. 30 (1995) 5743.

[265] U. Erb, Nanostruct. Mater. 6 (1995) 533.

[266] U. Erb, G. Palumbo, B. Szpunar, K.T. Aust, Nanostruct. Mater. 9 (1997) 261.

[267] K.J. Bryden, J.Y. Ying, Nanostruct. Mater. 9 (1997) 485.

[268] T. Yamasaki, P. Schloßmacher, K. Ehrlich, Y. Ogino, Nanostruct. Mater. 10 (1998) 375.

[269] M.T. Trudeau, Nanostruct. Mater. 12 (1999) 55.

[270] G. Hibbard, K.T. Aust, G. Palumbo, U. Erb, Scr. Meter. 44 (2001) 513.

[271] D.H. Jeong, F. Gonzalez, G. Palumbo, K.T. Aust, U. Erb, Scr. Mater. 44 (2001) 493.

[272] C.A. Schuh, T.G. Nieh, T. Yamasaki, Scr. Mater. 46 (2002) 735.

[273] H. Natter, M. Schmelzer, R. Hempelmann, J. Mater. Res. 13 (1998) 1186.

[274] C.B. Wang, D.L. Wang, W.X. Chen, Y.Y. Wang, Wear 253 (2002) 563.

[275] F. Ebrahimi, G.R. Bourne, M.S. Kelly, T.E. Matthews, Nanostruct. Mater. 11 (1999) 343.

[276] H. Li, F. Ebrahimi, Mater. Sci. Eng. A 347 (2003) 93.

[277] M.P. Seah, C. Leach, Phil. Mag. 31 (1975) 627.

[278] C.C. Roth, H. Leidheiser, J. Electrochem. Soc. 133 (1953) 2491.

[279] A. Robertson, U. Erb, G. Palumbo, Nanostruct. Mater. 12 (1999) 1035.

[280] G. Palumbo, F. Gonzalez, A.M. Brennenstuhl, U. Erb, W. Shmayda, P.C. Lichtenberger, Nanostruct. Mater. 9 (1997) 737.

[281] G.W. Nieman, J.R. Weertman, R.W. Siegel, Scr. Met. 23 (1989) 2013.

[282] G.W. Nieman, J.R. Weertman, R.W. Siegel, J. Mater. Res. 6 (1991) 1012.

[283] H.J. Hoffer, R.S. Averbach, Scr. Metall. Mater. 24 (1990) 2407.

[284] A.M. El-Sherik, U. Erb, G. Palumbo, K.T. Aust, Scr. Metall. Mater. 27 (1992) 1185.

[285] R.W. Siegel, G.E. Fougere, Nanostruct. Mater. 6 (1995) 205.

[286] A.H. Chokshi, A. Rosen, J. Karch, H. Gleiter, Scr. Metal. 23 (1989) 1679.

[287] K. Lu, W.D. Wei, J.T. Wang, Scr. Metall. Mater. 24 (1990) 2319.

[288] G.E. Fougere, J.R. Weertman, R.W. Siegel, S. Kim, Scr. Metall. Mater. 26 (1992) 1879.

[289] D.A. Konstantinidis, E.C. Aifantis, Nanostruct. Mater. 10 (1998) 1111.

[290] G. Palumbo, S.J. Thorpe, K.T. Aust, Scr. Metal. Mater. 24 (1990) 1347.

[291] C. Suryanarayana, D. Mukhopadhyay, S.N. Patankar, F.H. Froes, J. Mater. Res 7 (1992) 2114.

[292] P.G. Sanders, C.J. Youngdahl, J.R. Weertman, Mater. Sci. Eng. A 234-236 (1997) 77.

[293] S. Takeuchi, Scr. Mater. 44 (2001) 1483.

[294] X.K. Sun, J. Xu, W.X. Chen, W.D. Wei, Nanostruct. Mater. 4 (1994) 337.

[295] H. Hahn, P. Mondal, K.A. Padmanabhan, Nanostruct. Mater. 9 (1997) 603.

[296] H. Hahn, K.A. Padmanabhan, Phil. Mag. B 76 (1997) 559. 
[297] H. Van Swygenhoven, D. Farkas, A. Caro, Phys. Rev. B 62 (2000) 831.

[298] H. Van Swygenhoven, M. Spaczer, A. Caro, Acta Mater. 47 (1999) 3117.

[299] H. Van Swygenhoven, A. Caro, Phys. Rev. B 58 (1998) 11246.

[300] H. Van Swygenhoven, M. Spaczer, A. Caro, D. Farkas, Phys. Rev. B 60 (1999) 22.

[301] H. Van Swygenhoven, A. Caro, Nanostruct. Mater. 9 (1999) 669.

[302] H. Van Swygenhoven, P.M. Derlet, Phys. Rev. B 64 (2001) 224105.

[303] H. Van Swygenhoven, P.M. Derlet, A. Hasnaoui, Phys. Rev. B 66 (2002) 024101.

[304] J. Schiotz, F.D. Di Tolla, K.W. Jacobsen, Nature 391 (1998) 561.

[305] J. Schiotz, T. Verge, F.D. Di Tolla, K.W. Jacobsen, Phys. Rev. B 60 (1999) 11971.

[306] P. Keblinski, S.R. Phillpot, D. Wolf, H. Gleiter, Acta Mater. 45 (1997) 987.

[307] P. Keblinski, D. Wolf, H. Gleiter, Interf. Sci. 6 (1998) 205.

[308] V. Yamakov, D. Wolf, S.R. Phillpot, H. Gleiter, Acta Mater. 50 (2002) 61.

[309] H.S. Kim, M.B. Bush, Nanostruct. Mater. 11 (1999) 361.

[310] H.S. Kim, Y. Estrin, M.B. Bush, Acta Mater. 48 (2000) 493.

[311] H.H. Fu, D.J. Benson, M.A. Meyers, Acta Mater. 49 (2001) 2567.

[312] P. Sharma, S. Ganti, J. Mater. Res. 18 (2003) 1823.

[313] L.A. Ovid'ko, Science 295 (2002) 2386.

[314] M. Murayama, J.M. Howe, H. Hidaka, S. Takaki, Science 295 (2002) 2433.

[315] M. Yu Gutkin, A.L. Kolesnikova, L.A. Ovid'ko, N.V. Skiba, J. Metast. Nanocryst. Mater. 12 (2002) 47.

[316] M. Yu Gutkin, L.A. Ovid'ko, N.V. Skiba, Acta Mater. 51 (2003) 4059.

[317] M. Ke, S.A. Hackney, W.W. Milligan, E.C. Aifantis, Nanostruct. Mater. 5 (1995) 689.

[318] T.H. Courtney, Mechanical Behavior of Materials, McGraw-Hill, New York, 1990 (pp. 302-307).

[319] A.K. Mukherjee, Mater. Sci. Eng. A 322 (2002) 1.

[320] F.A. Mohamed, Y. Li, Mater. Sci. Eng. A 298 (2001) 1.

[321] T.G. Nieh, J. Wadsworth, Scr. Metall. Mater. 28 (1993) 1119.

[322] R.S. Mishra, T.R. Bieler, A.K. Mukherjee, Acta Mater. 45 (1997) 561.

[323] K. Matsuki, H. Sugahara, T. Aida, N. Takatsuji, J. Kusui, K. Yokoe, Mater. Trans. JIM 40 (1999) 737.

[324] L. Lu, M.L. Sui, K. Lu, Science 287 (2000) 1463.

[325] R.S. Mishra, R.Z. Valiev, A.K. Mukherjee, Nanostruct. Mater. 9 (1997) 473.

[326] S.X. Mcfadden, R.S. Mishra, R.Z. Valiev, A.P. Zhilyaev, A.K. Mukherjee, Nature 398 (1999) 684.

[327] S.X. Mcfadden, A.V. Sergueeva, R.S. Mishra, A.K. Mukherjee, Mater. Sci. Technol. 16 (2000) 1340.

[328] S.X. Mcfadden, R.Z. Valiev, A.K. Mukherjee, Mater. Sci. Eng. A 319-321 (2001) 849.

[329] M. Ciftcioglu, M.J. Mayo, in: M.J. Mayo, M. Kobayashi, J. Wadsworth (Eds.), Superplasticity in Metals, Ceramics and Intermetallics, Materials Research Society, Pittsburgh, 1990, pp. 77-86.

[330] H. Hahn, R.S. Averback, J. Am. Ceram. Soc. 74 (1991) 2918.

[331] C. Carry, A. Mocellin, Ceram. Int. 13 (1987) 89.

[332] M.J. Mayo, in: C.M. Chow, N.I. Noskova (Eds.), Nanostructures Materials: Science and Technology, Kluwer Academic Publishers, Dordrecht, 1997, pp. 361-385.

[333] P.G. Sanders, M. Rittner, E. Kiedaish, J.R. Weertman, H. Kung, Y.C. Lu, Nanostruct. Mater. 9 (1997) 433.

[334] B. Cai, Q.P. Kong, L. Lu, K. Lu, Mater. Sci. Eng. A 286 (2000) 188.

[335] N. Wang, Z. Wang, K.T. Aust, U. Erb, Mater. Sci. Eng. A 237 (1997) 150.

[336] A. Federov, M. Yu Gutkin, I.A. Ovid'ko, Scr. Mater. 47 (2002) 51.

[337] A.A. Federov, M. Yu Gutkin, I.A. Ovid'ko, Acta Mater. 51 (2003) 887.

[338] G. Gottstein, A.H. King, L.S. Shvindlerman, Acta Mater. 48 (2000) 397.

[339] G. Palumbo, U. Erb, K.T. Aust, Scr. Metall. Mater. 24 (1990) 2347.

[340] G. Palumbo, D.M. Doyle, A.M. El-Sherik, U. Erb, K.T. Aust, Scr. Metall. Mater. 25 (1991) 679.

[341] R.A. Masumura, P.M. Hazzledine, C.S. Pande, Acta Mater. 46 (1998) 4527.

[342] R.L. Coble, J. Appl. Phys. 34 (1963) 1697.

[343] S. Schumacher, R. Birringer, R. Strauss, H. Gleiter, Acta Metall. 37 (1989) 2485.

[344] W.M. Yin, S.H. Whang, R. Mirshams, C.H. Xiao, Mater. Sci. Eng. A 301 (2001) 18.

[345] N. Wang, Z. Wang, K.T. Aust, U. Erb, Acta Metall. Mater. 43 (1995) 519.

[346] K.S. Kumar, S. Suresh, M.F. Chisholm, J.A. Horton, P. Wang, Acta Mater. 51 (2003) 387.

[347] S.N.G. Chu, J.C.M. Li, J. Met. Ci. 12 (1977) 2200.

[348] F. Yang, J.C. Li, Scr. Mater. 32 (1995) 139.

[349] D.S. Stone, K.B. Yoder, J. Mater. Res. 9 (1994) 2524. 
[350] A.A. Elmustafa, J.A. Eastman, M.N. Ritter, J.R. Weertman, D.S. Stone, Scr. Mater. 43 (2000) 951.

[351] A.A. Elmustafa, D.S. Stone, Acta Metall. 50 (2002) 3641.

[352] A.A. Elmustafa, D.S. Stone, Mater. Lett. 57 (2003) 1072.

[353] A.A. Elmustafa, D.S. Stone, J. Mech. Phys. Solids 51 (2003) 357.

[354] F.A. McClintock, A.S. Argon, Mechanical Behavior of Materials, Addison-Wesley, New York, 1966 (pp. 176-177).

[355] M. Legros, B.R. Elliot, M.N. Rittner, J.R. Weertman, K.J. Hemker, Phil. Mag. A 80 (2000) 1017.

[356] C.J. Youngdahl, P.G. Sanders, J.A. Eastman, J.R. Weertman, Scr. Mater. 37 (1997) 809.

[357] L. Lu, L.B. Wang, B.Z. Ning, K. Lu, J. Mater. Res. 15 (2000) 270.

[358] F. Dalla Torre, H. Van, M. Swygnhoven, Victoria, Acta Mater. 50 (2002) 3957.

[359] E. Ma, Scr. Mater. 49 (2003) 663.

[360] C.C. Koch, Scr. Mater. 49 (2003) 657.

[361] D. Jia, K.T. Ramesh, E. Ma, Scr. Mater. 42 (2000) 73.

[362] Q.M. Wei, D. Jia, K.T. Ramesh, E. Ma, Appl. Phys. Lett. 81 (2002) 1240.

[363] D. Jia, K.T. Ramesh, E. Ma, Acta Mater. 51 (2003) 3495.

[364] G. Gray III, T.C. Lowe, C.M. Cady, R.Z. Valiev, I.V. Aleksandrov, Nanostruct. Mater. 9 (1997) 477.

[365] M.J. Mayo, R.W. Siegel, A. Narayanasamy, W.D. Nix, J. Mater. Res. 5 (1990) 1073.

[366] M.J. Mayo, R.W. Siegel, Y.X. Liao, W.D. Nix, J. Mater. Res. 7 (1992) 973.

[367] L. Lu, S.X. Li, K. Lu, Scr. Mater. 45 (2001) 1163.

[368] R. Schwaiger, B. Moser, M. Dao, N. Chollacoop, S. Suresh, Acta Mater. 51 (2003) 5159.

[369] C.J. Youngdahl, J.R. Weertman, R.C. Hugo, H.H. Kung, Scr. Mater. 44 (2001) 1475.

[370] M.J. Mayo, in: M. Nastasi, D.N. Parkin, H. Gleiter (Eds.), Mechanical Properties and Deformation Behavior of Materials Having Ultra-Fine Microstructures, Kluwer Academic Publishers, London, 1994, pp. 361-380.

[371] R.W. Siegel, S. Ramasamy, H. Hann, Z. Li, T. Lu, R. Gronsky, J. Mater. Res. 3 (1988) 1376.

[372] J. Karch, R. Birringer, H. Gleiter, Nature 330 (1987) 556.

[373] M. Jimenez-Melendo, A. Dominguez-Rodriguez, A. Bravo-Leon, J. Am. Ceram. Soc. 81 (1998) 2761.

[374] T.G. Nieh, J. Adsworth, Acta Metall. Mater. 38 (1990) 1121.

[375] F. Wakai, S. Sakaguchi, Y. Matsuno, Adv. Ceram. Mater. 259 (1986) 1.

[376] A. Bravo-Leon, M. Jimenez-Melendo, A. Dominguez-Rodriguez, Acta Metall. Mater. 40 (1992) 2717.

[377] T.G. Langdon, JOM 42 (1990) 8.

[378] K. Kajihara, Y. Yoshizawa, T. Sakuma, Scr. Metall. Mater. 28 (1993) 559.

[379] T. Sakuma, Mater. Sci. Forum 243-245 (1997) 327.

[380] M.J. Mayo, Nanostruct. Mater. 9 (1997) 717.

[381] L. Chen, T. Rouxel, R. Chaim, H. Vesteghem, D. Sherman, Mater. Sci. Forum 243-245 (1997) 245.

[382] H. Hahn, R.S. Averback, J. Am. Ceram. Soc. 74 (1991) 2918.

[383] T. Hanlon, Y. Kwon, S. Suresh, Scr. Mater. 49 (2003) 675.

[384] V. Patlan, K. Higashi, K. Kitagawa, M. Kawazoe, Mater. Sci. Eng. A 319 (2001) 587.

[385] C.S. Chung, J. Kim, H. Kim, W. Kim, Mater. Sci. Eng. A 337 (2002) 39.

[386] H. Holleck, V. Schier, Surf. Coat. Technol. 76-77 (1995) 328.

[387] S.C. Tjong, J.S. Ku, C.S. Wu, Scr. Metall. Mater. 31 (1994) 835.

[388] S.C. Tjong, Thin Solid Films 274 (1996) 95.

[389] S.C. Tjong, J.S. Ku, N.J. Ho, Mater. Sci. Technol. 13 (1997) 56.

[390] A. Kulkarni, S. Sampath, A. Goland, H. Herman, B. Dowd, Scr. Mater. 43 (2000) 471.

[391] Y. Liu, T.E. Fischer, A. Dent, Surf. Coat. Technol. 167 (2003) 68.

[392] L. Zhao, C. Herbst-Dederichs, E. Lugscheider, Adv. Eng. Mater. 4 (2002) 922.

[393] Y.C. Zhu, K. Yukimura, C.X. Ding, P.Y. Zhang, Thin Solid Films 388 (2001) 277.

[394] E.H. Jordan, M. Gell, Y.H. Sohn, D. Goberman, L. Shaw, S. Jiang, M. Wang, T.D. Xiao, Y. Wang, P. Strutt, Mater. Sci. Eng. A 301 (2001) 80.

[395] T. Grosdidier, A. Tidu, H.L. Liao, Scr. Mater. 44 (2001) 387.

[396] H. Chen, Y. Zhang, C. Ding, Wear 253 (2002) 885.

[397] H. Luo, D. Goberman, L. Shaw, M. Gell, Mater. Sci. Eng. A 346 (2003) 237.

[398] X. Lin, Y. Zeng, X. Zhou, C. Ding, Mater. Sci. Eng. A 357 (2003) 228.

[399] P. Bansal, N.P. Padture, A. Vasiliev, Acta Mater. 51 (2003) 2959.

[400] H.G. Jiang, M.L. Lau, E.J. Lavernia, Nanostruct. Mater. 10 (1998) 169.

[401] M.M. Lau, V.V. Gupta, E.J. Lavernia, Nanostruct. Mater. 12 (1999) 319.

[402] J. He, M. Ice, S. Dallek, E.J. Lavernia, Metall. Mater. Trans. A 31 (2000) 541. 
[403] H. Jiang, M. Lau, V.L. Tellkamp, E.J. Lavernia, in: H.S. Nalwa (Ed.), Handbook of Nanostructured Materials and Nanotechnology, vol. 1, Academic Press, New York, 2000 (pp. 159-213, Chapter 3).

[404] T.M. Yonushonis, J. Therm. Spray Technol. 6 (1997) 50.

[405] Y.H. Lu, Z.F. Zhou, P. Sit, Y.G. Shen, K.Y. Li, H. Chen, Thin Solid Films, in press.

[406] Y.H. Lu, P. Sit, Z.F. Zhou, Y.G. Shen, K.Y. Li, H. Chen, Surf. Coat. Technol., in press.

[407] C. Berg, C. Friedrich, E. Broszeit, C. Berger, Surf. Coat. Technol. 86-87 (1996) 184.

[408] J.H. Hsieh, C. Liang, C.H. Yu, W. Wu, Surf. Coat. Technol. 108-109 (1998) 132.

[409] S.S. Eskildsen, C. Mathiasen, M. Foss, Surf. Coat. Technol. 116-119 (1999) 18.

[410] S. Wilson, T. Alpas, Wear 245 (2000) 223.

[411] R. Wiedemann, H. Oettel, T. Bertram, V. Weihnacht, Adv. Eng. Mater. 3 (2001) 865.

[412] A.A. Galska, J.C. Uht, P.M. Adaias, J. Vac. Sci. Technol. A 6 (1988) 99.

[413] S.C. Tjong, N.B. Wong, G. Li, S.T. Lee, Mater. Chem. Phys. 62 (2000) 241.

[414] S.C. Tjong, H.P. Ho, S.T. Lee, Diamond Rel. Mater. 10 (2001) 1578.

[415] J.H. Huang, C.H. Lin, H. Chen, Mater. Chem. Phys. 59 (1999) 49.

[416] C. Mitterer, P.H. Maryhofer, W. Waldhauser, E. Kelesoglu, P. Losbichler, Surf. Coat. Technol. 108-109 (1998) 230.

[417] T. Sikola, J. Spousta, R. Ceska, J. Zlamal, L. Dittrichova, A. Nebojsa, K. Navratil, D. Rafaja, J. Zemek, V. Perina, Surf. Coat. Technol. 108-109 (1998) 284.

[418] K. Volz, M. Kiuchi, W. Ensinger, Surf. Coat. Technol. 108-109 (1998) 303.

[419] B.F. Chen, W.L. Pan, G.P. Yu, J.H. Hwang, J.H. Huang, Surf. Coat. Technol. 111 (1999) 16.

[420] W.J. Chou, G.P. Yu, J.H. Huang, Surf. Coat. Technol. 140 (2001) 206.

[421] W.J. Chou, G.P. Yu, J.H. Huang, Surf. Coat. Technol. 149 (2002) 7.

[422] D.G. Kim, T.Y. Seong, Y.J. Baik, Surf. Coat. Technol. 153 (2002) 79.

[423] S.M. Aouadi, F. Namavar, T.Z. Gorishnyy, S.L. Rohde, Surf. Coat. Technol. 160 (2002) 145.

[424] C.H. Ma, J.H. Huang, H. Chen, Thin Solid Films 446 (2003) 184.

[425] C.H. Ma, J.H. Huang, H. Chen, Thin Solid Films 418 (2002) 73.

[426] C.H. Ma, J.H. Huang, H. Chen, Surf. Coat. Technol., in press.

[427] U. Wahlstrom, L. Hultman, J.E. Sundgren, F. Adibi, I. Petrov, J.E. Greene, Thin Solid Films 235 (1993) 62.

[428] R. Wuhrer, W.Y. Yeung, M.R. Philips, G. McCredie, Thin Solid Films 290 (1996) 339.

[429] H. Jensen, U.M. Jensen, G. Sorensen, Surf. Coat. Technol. 74-75 (1995) 297.

[430] F.A. Schmidt, Int. Mater. Rev. 35 (1990) 61.

[431] J.E. Greene, Handbook of Crystal Growth, vol. 1, Elsevier, New York, 1993 (p. 641).

[432] W. Ensinger, Nucl. Inst. Meth. Phys. Res. B 127-128 (1997) 796.

[433] H. Ljungerantz, M. Oden, L. Huttman, J.E. Greene, J.E. Sundgren, J. Appl. Phys. 80 (1996) h6725.

[434] C.T. Chen, Y.C. Song, G.P. Yu, J.H. Huang, J. Mater. Eng. Perform. 7 (1998) 324.

[435] J.E. Greene, J.E. Sundgren, L. Hultman, I. Petrov, D.B. Bergstrom, Appl. Phys. Lett. 67 (1995) 2928.

[436] L. Hultman, J.E. Sundgren, J.E. Greene, D.B. Bergstrom, I. Petrov, J. Appl. Phys. 78 (1995) 5395.

[437] J.A. Thorton, D.W. Hoffman, Thin Solid Films 171 (1989) 5.

[438] R. Messier, A.P. Giri, R.A. Roy, J. Vac. Sci. Technol. A 2 (1984) 500.

[439] C.R.M. Grovenor, H.T.G. Hentzell, D.A. Smith, Acta Metall. 32 (1984) 773.

[440] Y. Sakuragi, Y. Watanabe, Y. Amamoto, Y. Nakamura, J. Mater, Sci. Mater. Electron. 10 (1999) 533.

[441] W. Ensinger, Nucl. Instrum. Methods Phys. Res. B 127-128 (1997) 796.

[442] Chemistry Webelement, http://www.webelements.com.

[443] J.S. Koehler, Phys. Rev. B 2 (1970) 547.

[444] H. Holleck, J. Vac. Sci. Technol. A 4 (1986) 2661.

[445] S.L. Lehoczy, J. Appl. Phys. 49 (1978) 5479.

[446] S.L. Lehoczy, Phys. Rev. Lett. 41 (1978) 1814.

[447] M. Setoyama, A. Nakayama, M. Tanaka, N. Kitagawa, T. Nomura, Surf. Coat. Technol. 86-87 (1996) 225.

[448] U. Helmersson, S. Todorova, S.A. Barnett, J.E. Sundgren, L.C. Markert, J.E. Greene, J. Appl. Phys. 62 (1987) 481.

[449] M. Shinn, L. Hultman, S.A. Barnett, J. Mater. Res. 7 (1992) 901.

[450] X. Chu, M.S. Wong, W.D. Sproul, S.L. Rohde, S.A. Barnett, J. Vac. Sci. Technol. A 10 (1992) 1604.

[451] X. Chu, S.A. Barnett, J. Appl. Phys. 77 (1995) 4403.

[452] H. Wrzesinska, J. Ratajczak, K. Studzinska, J. Katcki, Mater. Chem. Phys. 81 (2003) 265.

[453] S. Veprek, S. Reiprich, S. Li, Appl. Phys. Lett. 66 (1995) 2640.

[454] S. Veprek, S. Reiprich, Thin Solid Films 268 (1995) 64.

[455] S. Veprek, M. Haussmann, S. Reiprich, J. Vac. Sci. Technol. A 14 (1996) 46. 
[456] S. Veprek, M. Haussmann, S. Reiprich, S. Li, J. Dian, Surf. Coat. Technol. 86-87 (1996) 394.

[457] S. Veprek, P. Nesladek, A. Niederhofer, F. Glatz, M. Jilek, M. Sima, Surf. Coat. Technol. 108-109 (1998) 138.

[458] S. Christiansen, M. Albrecht, H.P. Strunk, S. Veprek, J. Vac Sci. Technol. B 16 (1998) 19.

[459] S. Veprek, J. Vac. Sci. Technol. A 17 (1999) 2401.

[460] H.D. Mannling, D.S. Patil, K. Moto, M. Jilek, S. Veprek, Surf. Coat. Technol. 146-147 (2001) 263.

[461] P. Karvankova, M.G.J. Verprek-Heijman, O. Zindulka, A. Bergmaier, S. Veprek, Surf. Coat. Technol. $163-164$ (2003) 149.

[462] S. Veprek, S. Mukherjee, H.D. Mannling, J. He, Mater. Sci. Eng. A 340 (2003) 292.

[463] A.A. Voevodin, S.V. Prasad, J.S. Zabinski, J. Appl. Phys. 82 (1997) 855.

[464] A.A. Voevodin, J.S. Zabinski, J. Mater. Sci. 33 (1998) 319.

[465] A.A. Voevodin, J.P. O’Neill, S.V. Prasad, J.S. Zabinski, J. Vac. Sci. Technol. 17 (1999) 986.

[466] R. Maboudian, Surf. Sci. Rep. 30 (1998) 207.

[467] R. Maboudia, R.T. Howe, J. Vac. Sci. Technol. B 15 (1997) 1.

[468] R. Maboudia, R.T. Howe, Tribol. Lett. 3 (1997) 215.

[469] C.R. Stoldt, M.C. Fritz, C. Carraro, R. Maboudian, Appl. Phys. Lett. 79 (2001) 347.

[470] A.R. Krauss, O. Aucillo, D.M. Gruen, A. Jayatissa, A. Sumant, J. Tucek, D.C. Mancini, N. Moldovan, A. Erdemir, D. Ersoy, M. Gardos, H.G. Busmann, E.M. Meyer, M.Q. Ding, Diamond Rel. Mater. 10 (2001) 1952.

[471] H.D. Espinosa, B. Peng, B.C. Provok, N. Moldovan, O. Auciello, J.A. Carlisle, D.M. Gruen, D.C. Mancini, J. Appl. Phys. 94 (2003) 6076.

[472] A. Erdemir, G.R. Fenske, A.R. Krauss, D.M. Gruen, T. McCauley, R.T. Csencsits, Surf. Coat. Technol. 120-121 (1999) 565.

[473] S. Jiao, A. Sumant, M.A. Kirk, D.M. Gruen, A.R. Krauss, O. Auciello, J. Appl. Phys. 90 (2001) 118.

[474] S. Bhattacharyya, O. Auciello, J. Birrell, J.A. Carlislle, L.A. Curtiss, A.N. Goyette, D.M. Gruen, A.R. Krauss, J. Schlueter, A. Sumant, P. Zapol, Appl. Phys. Lett. 79 (2001) 1441.

[475] M.Q. Ding, O. Auciello, J.A. Carlisle, D.M. Gruen, J. Vac. Sci. Technol. B 21 (2003) 1644. 